Prepared for the U.S. Department of Energy

under Contract DE-AC05-76RL01830

\title{
Water Quality Sampling Locations Along the Shoreline of the Columbia River, Hanford Site, Washington
}

RE Peterson

GW Patton

December 2009

\section{Pacific Northwest}

NATIONAL LABORATORY

Proudly Opercted by Battelle Since 1965 


\title{
DISCLAIMER
}

This report was prepared as an account of work sponsored by an agency of the United States Government. Neither the United States Government nor any agency thereof, nor Battelle Memorial Institute, nor any of their employees, makes any warranty, express or implied, or assumes any legal liability or responsibility for the accuracy, completeness, or usefulness of any information, apparatus, product, or process disclosed, or represents that its use would not infringe privately owned rights. Reference herein to any specific commercial product, process, or service by trade name, trademark, manufacturer, or otherwise does not necessarily constitute or imply its endorsement, recommendation, or favoring by the United States Government or any agency thereof, or Battelle Memorial Institute. The views and opinions of authors expressed herein do not necessarily state or reflect those of the United States Government or any agency thereof.

\author{
PACIFIC NORTHWEST NATIONAL LABORATORY \\ operated by \\ BATTELLE \\ for the \\ UNITED STATES DEPARTMENT OF ENERGY \\ under Contract DE-AC05-76RL01830
}

Printed in the United States of America

Available to DOE and DOE contractors from the

Office of Scientific and Technical Information,

P.O. Box 62, Oak Ridge, TN 37831-0062;

ph: (865) 576-8401

fax: $(865)$ 576-5728

email: reports@adonis.osti.gov

\footnotetext{
Available to the public from the National Technical Information Service, U.S. Department of Commerce, 5285 Port Royal Rd., Springfield, VA 22161 ph: (800) 553-6847 fax: (703) 605-6900 email: orders@ntis.fedworld.gov

online ordering: http://www.ntis.gov/ordering.htm
} 


\section{Water Quality Sampling Locations Along the Shoreline of the Columbia River, Hanford Site, Washington}

RE Peterson

GW Patton

December 2009

Prepared for

the U.S. Department of Energy

under Contract DE-AC05-76RL01830

Pacific Northwest National Laboratory

Richland, Washington 99352 



\section{Summary}

As environmental monitoring evolved on the Hanford Site, several different conventions were used to name or describe location information for various sampling sites along the Hanford Reach of the Columbia River. These conventions range from handwritten descriptions in field notebooks to the use of modern electronic surveying equipment, such as global positioning system receivers. Because of the diverse methods, inconsistent archiving of analytical results in various electronic databases and published reports occurred, including use of multiple names for the same site and inaccurate location information.

This document provides listings of sampling sites that are associated with groundwater and river water sampling. The report identifies names and locations for sites associated with sampling: (a) nearriver groundwater using aquifer sampling tubes; (b) riverbank springs and seepage areas; (c) pore water collected from riverbed sediment; and (d) Columbia River water. Included in the listings are historical names used for a particular site and the best available geographic coordinates for the site, as of 2009.

In an effort to create more consistency in the descriptive names used for water quality sampling sites, a naming convention is proposed in this document. The convention assumes that a unique identifier is assigned to each site that is monitored and that this identifier serves electronic database management requirements. The descriptive name is assigned for the convenience of the particular project and subsequent data user. As the historical database is used more intensively, this document may be revised as a consequence of discovering potential errors and the need to gain consensus on a proposed naming convention for some water quality monitoring sites. 



\section{Acknowledgments}

This report has drawn on the efforts of numerous individuals who have worked along the Columbia River shoreline at the Hanford Site, to whom the authors extend their sincere appreciation. Of particular help in sorting out the locations and names associated with riverbank springs were Roger Dirkes, ${ }^{1}$ who provided detailed recollections of his early work on the river, and Richard Mahood, ${ }^{2}$ who spent countless days in the field documenting locations and collecting samples. Rich was also the principal developer of methods to install aquifer sampling tubes along the shoreline and provided leadership to several campaigns that resulted in the comprehensive monitoring network available today.

William Webber ${ }^{2}$ and JoAnne Rieger ${ }^{2}$ engaged the senior author in extensive discussions regarding site location information from the perspective of database developers, which certainly helped guide the level of detail provided in this report. JoAnne patiently responded to numerous requests for assistance in querying existing databases, for which the authors are grateful. Lynn Bisping ${ }^{1}$ graciously provided peer review of the report and offered numerous suggestions that improved clarity and accuracy. Kyle Parker ${ }^{1}$ developed the location maps found in the appendix, using coverages available from the Hanford Site's central mapping services. His efforts to assemble this geographic information system provides a very useful tool for shoreline investigations. Mike Parker ${ }^{1}$ masterfully put all this material together in a report format that will prove useful to numerous Hanford Site field workers and database management staff.

Finally, the authors would like to thank in advance those who choose to respond to the proposal to develop more consistent conventions for descriptive names for monitoring sites. We also appreciate feedback on any errors and omissions that are discovered.

Bob Peterson and Greg Patton

December 2009

\footnotetext{
${ }^{1}$ Pacific Northwest National Laboratory

${ }^{2} \mathrm{CH} 2 \mathrm{M}$ Hill Plateau Remediation Company
} 



\section{Acronyms and Abbreviations}

BHI

BNWL

CERCLA

CHPRC

DOE

DOE-RL

$\mathrm{DOH}$

Ecology

EPA

ERC

GIS

GPS

GW

HEIS

HGIS

HRM

HWIS

PNL

PNNL

PSRPP

RCBRA

RCRA

SESP

SW

USGS

TPA

Tri-Parties

WAC

WCH

WHC

WIDL

WIDS
Bechtel Hanford, Inc.

Battelle Northwest Laboratory

Comprehensive Environmental Response, Compensation, and Liability Act of 1980

CH2M HILL Plateau Remediation Company

U.S. Department of Energy

U.S. Department of Energy, Richland Operations Office

Washington State Department of Health

Washington State Department of Ecology

U.S. Environmental Protection Agency

Environmental Restoration Contractor

geographic information system

global positioning system

groundwater media code (HEIS)

Hanford Environmental Information System

Hanford Geographic Information System

Hanford River Mile (pre-1999); Hanford River Marker (post-1999)

Hanford Well Information System

Pacific Northwest Laboratory (prior to 1995)

Pacific Northwest National Laboratory (post-1995)

Public Safety and Resource Protection Program

River Corridor Baseline Risk Assessment

Resource Conservation and Recovery Act of 1976

Surface Environmental Surveillance Project

surface water media code (HEIS)

U.S. Geological Survey

Hanford Federal Facility Agreement and Consent Order (Tri-Party Agreement)

DOE, EPA, and Ecology

Washington State Administrative Code

Washington Closure Hanford, LLC

Westinghouse Hanford Company

Well Information and Document Lookup (user interface with HWIS)

Waste Information Data System 



\section{Contents}

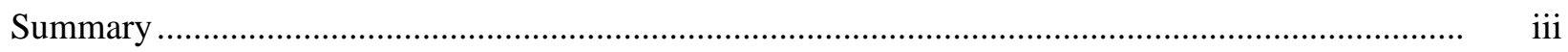

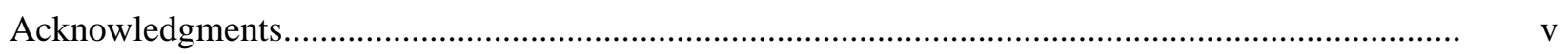

Acronyms and Abbreviations .............................................................................................. vii

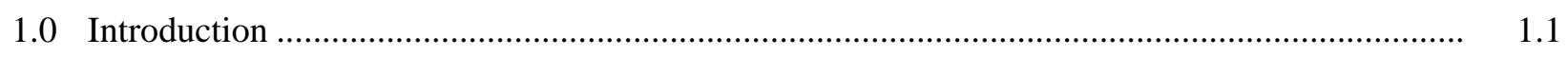

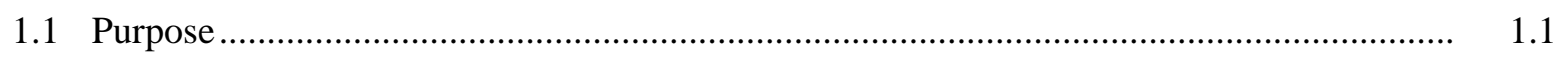

1.2 River-Related Water Quality Monitoring Activities ...................................................... 1.2

1.3 Geographic Referencing Systems ........................................................................... 1.3

1.3.1 Hanford River Miles/Markers and Shoreline Segments........................................ 1.3

1.3.2 Columbia River Channel Centerline ............................................................... 1.4

1.3.3 Aerial Photographs ....................................................................................... 1.4

2.0 Historical Investigations and Previous Work ................................................................... 2.1

2.1 Sitewide Environmental Surveillance Under Pacific Northwest Laboratory ...................... 2.1

2.2 Environmental Restoration Under Westinghouse Hanford Company ............................... 2.2

2.3 Environmental Restoration Under Bechtel Hanford, Inc. ................................................ 2.4

2.4 Soil and Groundwater Remediation Under Fluor Hanford, Inc. and CH2M Hill Plateau Remediation Company ........................................................................................ 2.5

2.5 River Corridor Baseline Risk Assessment Under Washington Closure Hanford, LLC ....... 2.5

2.6 Miscellaneous Investigations .................................................................................. 2.6

3.0 Aquifer Sampling Tubes.........................................................................................

3.1 Aquifer Sampling Tube Naming Conventions ............................................................... 3.1

3.2 Aquifer Sampling Tube Location Names and Coordinates............................................... 3.2

3.3 Previous Names for Aquifer Tubes .............................................................................. 3.2

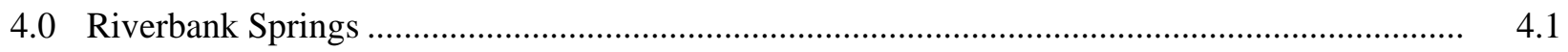

4.1 Riverbank Springs Naming Conventions ................................................................. 4.1

4.2 Riverbank Spring Names and Coordinates ................................................................ 4.2

4.3 Riverbank Springs: Previous Names ................................................................................ 4.3

5.0 Seep Wells in Rip Rap at N-Springs Shoreline .............................................................. 5.1

5.1 N-Springs Seep Well Names and Coordinates............................................................ 5.1

5.2 N-Springs Seep Well Name Usage .......................................................................... 5.1

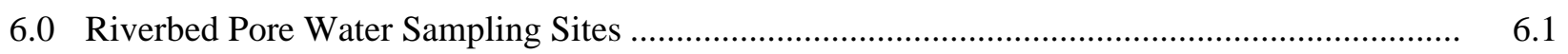

6.1 Riverbed Pore Water Site Naming Conventions ......................................................... 6.1

6.2 Pore Water Sampling Site Names and Coordinates ....................................................... 6.2

6.3 Pore Water Transect Reference Pins .......................................................................... 6.2

7.0 Columbia River Water Sampling Locations..................................................................

7.1 Columbia River Water Sampling Locations and Coordinates ......................................... 7.1

7.2 River Stage Recorders .................................................................................................. $\quad 7.2$

8.0 Proposed Naming Convention for Shoreline Water Quality Monitoring Sites .......................... 8.1 
8.1 Site Identifiers and Descriptive Site Names................................................................. 8.1

8.2 Proposed Convention for Descriptive Site Names ........................................................... 8.2

8.3 Examples of Proposed Site Names.................................................................................... 8.2

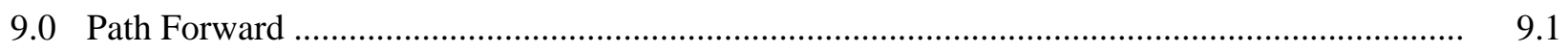

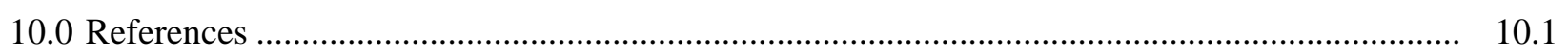

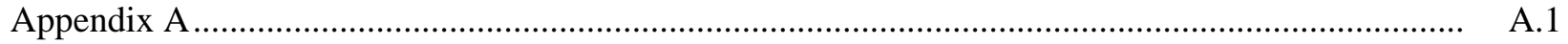




\section{Figures}

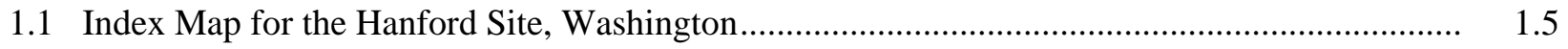

1.2 Illustration Showing Principal Features and Various Types of Water Quality Monitoring Sites Along the Hanford Reach............................................................................................ 1.6

1.3 Example of 1976 Aerial Photography Showing Low River Stage Conditions ........................... 1.6

2.1 Illustration Showing Mounding of Water Table at 100-K Area During Operations ................... 2.6

2.2 Aerial Photograph Showing Surface Runoff Across Shoreline at 100-K Area During Operations in 1955 ............................................................................................................ 2.7

2.3 Aerial Photograph Showing 100-K Area During Operations in 1965 _........................................ 2.7

2.4 Locations Along 100-H Area Where Riverbed Pore Water Samples were Collected in March 1995

2.5 Locations Along 100-D Area Where Riverbed Pore Water Samples were Collected in October/November 1995.

3.1 Illustration Showing Installation of Aquifer Sampling Tubes ................................................

4.1 Photograph of Riverbank Spring Site ................................................................................... 4.3

5.1 Areas of Riverbank Springs at 100-N Area During Operations ............................................... 5.2

5.2 Locations of Seep Wells and Seepage Spots Along 100-N Area Shoreline .............................. 5.3

5.3 Photograph of Seep Well Installed in Rip Rap Along 100-N Area Shoreline ........................... 5.3

6.1 Diagram of Riverbed Pore Water Sampling Apparatus............................................................. 6.3

7.1 Index Map for Columbia River Water Sampling Sites ........................................................

\section{Tables}

1.1 Hanford River Marker System Signposts

3.1 Aquifer Sampling Tube Sites Along the Hanford Site Shoreline ............................................. 3.4

3.2 Historical Names Used for Some Aquifer Sampling Tube Sites ............................................. 3.20

4.1 Riverbank Springs Observed Along the Hanford Site Shoreline of the Columbia River ............ 4.4

4.2 Historical Names Used for Riverbank Springs .................................................................... 4.10

5.1 Riverbank Seepage Well Casings Along the N-Springs Shoreline, Hanford Site ...................... 5.4

5.2 Historical Names for Riverbank Seepage Well Casings Sites ................................................. 5.5

6.1 Riverbed Substrate Pore Water Sampling Sites Along the Hanford Site Shoreline .................... 6.4

6.2 Transect Locator Pins for Riverbed Pore Water Sampling Sites ............................................ 6.10

7.1 Columbia River Water Sampling Locations in the Vicinity of the Hanford Site ....................... 7.4

7.2 River Stage Recorder Locations Along the Hanford Reach ...................................................

8.1 Code for Naming Water Quality Sampling Sites Along the Hanford Reach.............................. 8.3 



\subsection{Introduction}

This report contains an inventory of water quality sampling locations along the Columbia River as it flows across the Hanford Site, i.e., the "Hanford Reach" (Figure 1.1). The inventory addresses a need created by former inconsistent use of sampling site names and inaccurate or undocumented location information for various monitoring activities. The importance of historical and newly collected monitoring data is increasing as a consequence of environmental restoration efforts throughout the Columbia River corridor at the Hanford Site, and also because of the recent designation of the Hanford Reach as a National Monument (65 FR 114 2000). Monitoring data from the river environment contribute to a better understanding of the features and processes of the natural system, through which contamination may be dispersed.

In the past, numerous methods have been used to describe river-related sampling locations and features since monitoring activities began with the startup of reactor operations in the mid-1940s. These methods range from handwritten descriptions in field notebooks to use of modern electronic surveying equipment, such as global positioning system (GPS) receivers. The diverse methods resulted in inconsistent archiving of analytical results in electronic databases and published reports because of multiple names occasionally being used for the same site and inaccurate location information.

This report provides listings of water quality sampling sites that are associated with the groundwater/river interface. The listings provide names and locations for the following types of sampling sites: (a) near-river groundwater using aquifer sampling tubes; (b) riverbank springs and seepage areas; (c) pore water collected from riverbed sediment; and (d) Columbia River water. Figure 1.2 illustrates these various types of sampling sites. Included in the listings are historical names used for a particular site and the best available geographic coordinates for the site.

The information contained in this report has evolved during the early part of the $21^{\text {st }}$ century as a consequence of activities being conducted under a variety of environmental programs, including the U.S. Department of Energy's (DOE) Environmental Management program and Public Safety and Resource Protection program. Detailed information on sampling activities in the Hanford Reach corridor is continuing to evolve as projects such as the River Corridor Baseline Risk Assessment (RCBRA) conduct comprehensive sampling efforts throughout the Hanford Reach. Also, the DOE and other agencies continue to support research activities on various aspects of the river environment, so new insight relevant to monitoring is frequently produced.

As the historical database is used more intensively, this document may need to be revised within a relatively short time period as a consequence of discovering potential errors, newly identified sampling locations and media, and a need to gain consensus on naming conventions for some water quality monitoring sites.

\subsection{Purpose}

This inventory of river-related water quality monitoring sites establishes a basis to remove, or at least decrease, existing limitations on accessing historical data stored in the Hanford Environmental Information System (HEIS) database. Historical and newly collected monitoring data will, thus, become 
more readily available for evaluation, manipulation, and display using electronic databases and computerbased geographic information systems.

In an effort to create more consistency in the descriptive names used for water quality sampling sites, a naming convention is proposed in Chapter 8 . The convention assumes that a unique identifier is assigned to each site that is monitored, and that this identifier serves electronic database management requirements. The descriptive name is assigned for the convenience of the individual project and subsequent data users.

\subsection{River-Related Water Quality Monitoring Activities}

Environmental monitoring activities for the Hanford Site are described in an environmental monitoring plan (DOE-RL 2008a), which is updated every three years. Monitoring locations, schedules, and analysis suites for the river environment are described in the Pacific Northwest National Laboratory's (PNNL) annual Hanford Site Environmental Surveillance Master Sampling Schedule (e.g., Bisping 2009). Sampling and analysis activities associated with environmental restoration activities and regulatory compliance monitoring are described in project-specific monitoring/characterization plans.

PNNL's Surface Environmental Surveillance Project (SESP) collects samples at the following locations on a routine basis. Riverbank springs samples are collected annually at each of the six retired reactor areas (100 Area), the Hanford town site, and the 300 Area. Transect and near-shore samples of river water are collected quarterly at Vernita Bridge and the city of Richland's pumphouse, and annually at the 100-N Area, Hanford town site, and 300 Area. Continuous river water sampling systems are located at Priest Rapids Dam and the Richland pumphouse. Samples of Columbia River sediment are collected annually (typically after the peak spring runoff) from behind Priest Rapids and McNary Dams, and at White Bluffs Slough, 100-F Slough, Hanford Slough, selected riverbank springs, and a shoreline beach near the Richland pumphouse. The results of these activities are reported in the annual Hanford Site environmental report (e.g., Poston et al. 2009).

River water quality monitoring is also conducted by the U.S. Geological Survey (USGS) at Vernita Bridge and the Richland pumphouse on a quarterly basis, under subcontract to PNNL. In addition to sampling, the USGS measures river flow at a gauging station downstream from Priest Rapids Dam (USGS station \#12472800). Real-time stream flow data for this station are available at http://wa.water.usgs.gov. Additional current and historical river flow data are available at a website maintained by the University of Washington (http://www.cbr.washington.edu). The website contains a wide variety of river-related data.

The groundwater project, a part of the Soil and Groundwater Remediation Project currently managed by CH2M Hill Plateau Remediation Company (CHPRC), collects and evaluates samples from near-river wells, aquifer sampling tubes at the shoreline, and riverbank springs on at least an annual basis. This monitoring supports Comprehensive Environmental Response, Compensation, and Liability Act (CERCLA) environmental restoration activities, which include remedial investigations of waste sites and groundwater contamination, and performance evaluations associated with interim remedial actions. The groundwater project also collects groundwater data from near-river wells as part of regulatory requirements under the Resource Conservation and Recovery Act (RCRA). The results of groundwater 
monitoring activities are reported in an annual groundwater report (e.g., Hartman et al. 2009) and summarized in the annual Hanford Site environmental report (e.g., Poston et al. 2009).

\subsection{Geographic Referencing Systems}

As environmental monitoring evolved on the Hanford Site, several different conventions were used to provide location information for the various sampling sites along the Hanford Reach. This section summarizes some of those conventions. The summary starts with a description of the Hanford River Marker system, which has become part of the descriptive name for some sites, such as riverbank springs.

Geographic coordinates listed in this report are referenced to the Washington State Plane system, South Zone. The horizontal datum is the North American Datum of 1983 (NAD83) and the vertical datum is the North American Vertical Datum of 1988 (NAVD88). When conversion of historical data from the previously used vertical datum (i.e., NGVD29) has been necessary, an offset of 1.031 meters has been assumed (NAVD88 is higher than NGVD29). A factor of 3.2808 has been used for conversions from feet to meters. Coordinates may have been recorded in the field using latitude and longitude. When necessary, those coordinates have been converted to the Washington State Plane system using a conversion program maintained by the U.S. Army Corps of Engineers (“Corpscon;” available at http://crunch.tec.army.mil/software/corpscon/corpscon.html).

\subsubsection{Hanford River Miles/Markers and Shoreline Segments}

In the early 1980s, a student work crew from Pacific Northwest Laboratory (PNL) installed a series of 46 signposts along the Hanford Site shoreline from the Vernita Bridge (\#0) to Ferry Street in Richland, Washington (\#46). These signposts were installed to assist field crews, primarily those working from boats, in identifying environmental surveillance locations for the facilities side of the Hanford Site shoreline (i.e., right bank of the river). The signposts were spaced approximately 1.6 kilometers (1 mile) apart, as estimated by driving mileage; however, the actual distance between the signposts varies. The signposts were originally referred to as Hanford River "miles" but are now referred to as the Hanford River "markers," to avoid the impression that they represent exact one-mile intervals.

In 1999, a PNNL project was undertaken to locate the original signposts, survey their geographical coordinates using GPS receivers, and repair or replace damaged or missing signs. Most signs were successfully located and repaired, and some were moved farther onshore because of bank erosion. Where no signpost could be found, a new marker was installed at a position on the shoreline midway between existing upriver and downriver signposts. The updated reference system is referred to as Hanford River Markers (HRM) and the locations of signposts are shown on the maps in the Appendix. The upgraded system is referred to in the following site location tables as "HRM (2k)" to distinguish it from the HRM references made prior to the 1999 upgrade.

The coordinates for existing and original HRM signposts are listed in Table 1.1. The fields in the table are defined as follows:

- Hanford River Marker Name - Name used for the signpost. If an original signpost was replaced during the 1999 upgrade, the original signpost is listed at the end of the table as “\#_-old,” along with the original coordinates. 
- Segment - Segments defined to aid grouping monitoring sites by CERCLA groundwater operable units or other common characteristic.

- Northing and Easting - Horizontal coordinates in Washington State Plane (South Zone) system; units are meters; North American Datum of 1983.

- Coordinate Quality - Quality of the coordinates, either "estimated” from maps, or "surveyed” using global positioning system receivers and traditional land surveying methods.

- Coordinate Type - “GPS” refers to global positioning system equipment; some coordinates are estimated using GIS maps for the Hanford Site.

- Coordinate Reference - Report citation or other source information for coordinates.

- Site Description - Narrative description of the signpost location, as recorded during the 1999 upgrade project.

\subsubsection{Columbia River Channel Centerline}

A second reference system for position along the Columbia River is provided by a river channel centerline that appears on USGS topographic quadrangle maps and river navigation charts. This line represents the approximate center or deepest part (thalweg) of the channel. It includes mileage markers showing the distance upstream from the mouth of the river at Astoria, Oregon. Although this reference system has not been used in naming conventions for monitoring sites, it does provide a distance scale for river-channel related investigations. The centerline is shown on the maps provided in the Appendix.

\subsubsection{Aerial Photographs}

Abundant aerial photography of the Hanford Reach has been obtained over the years; however, providing a complete inventory of that coverage is beyond the scope of this report. One coverage that has been used extensively was obtained on April 10, 1976, as part of a project entitled "Observations on Juvenile Salmon Stranding in the Columbia River” (Contract No. 2311202335 with United Engineers and Contractors to Battelle, as part of a larger project with Washington Public Power Supply System, now Energy Northwest).

The color aerial photographs were obtained while the Columbia River was held at a constant discharge of approximately 50,000 cubic feet per second, a relatively low flow for the Hanford Reach. The actual shoreline for a subset of this coverage, which extends from 100-B Area downstream to the Hanford town site, was digitized by the Environmental Restoration Contractor during the 1990s and is used on various maps to indicate a low river stage shoreline, including the maps in this report. An example photograph from this set is shown in Figure 1.3. A mosaic of the photographs is available from the PNNL Ecology Group. The original negatives are archived by Lockheed Martin Information Services photography group, and are available as digitized images (^Photography LMSI@rl.gov; 509-376-3975). 


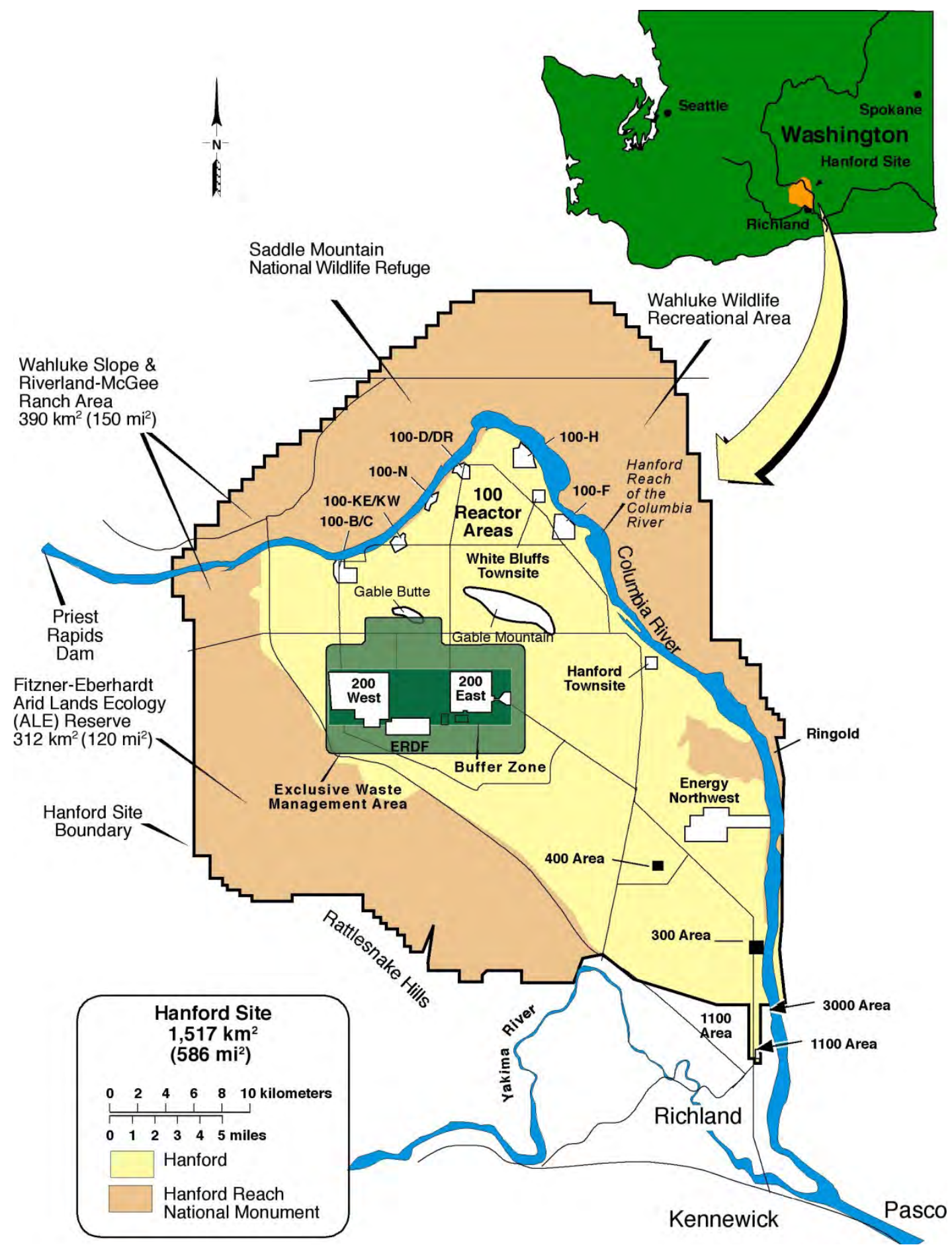

Figure 1.1. Index Map for the Hanford Site, Washington 


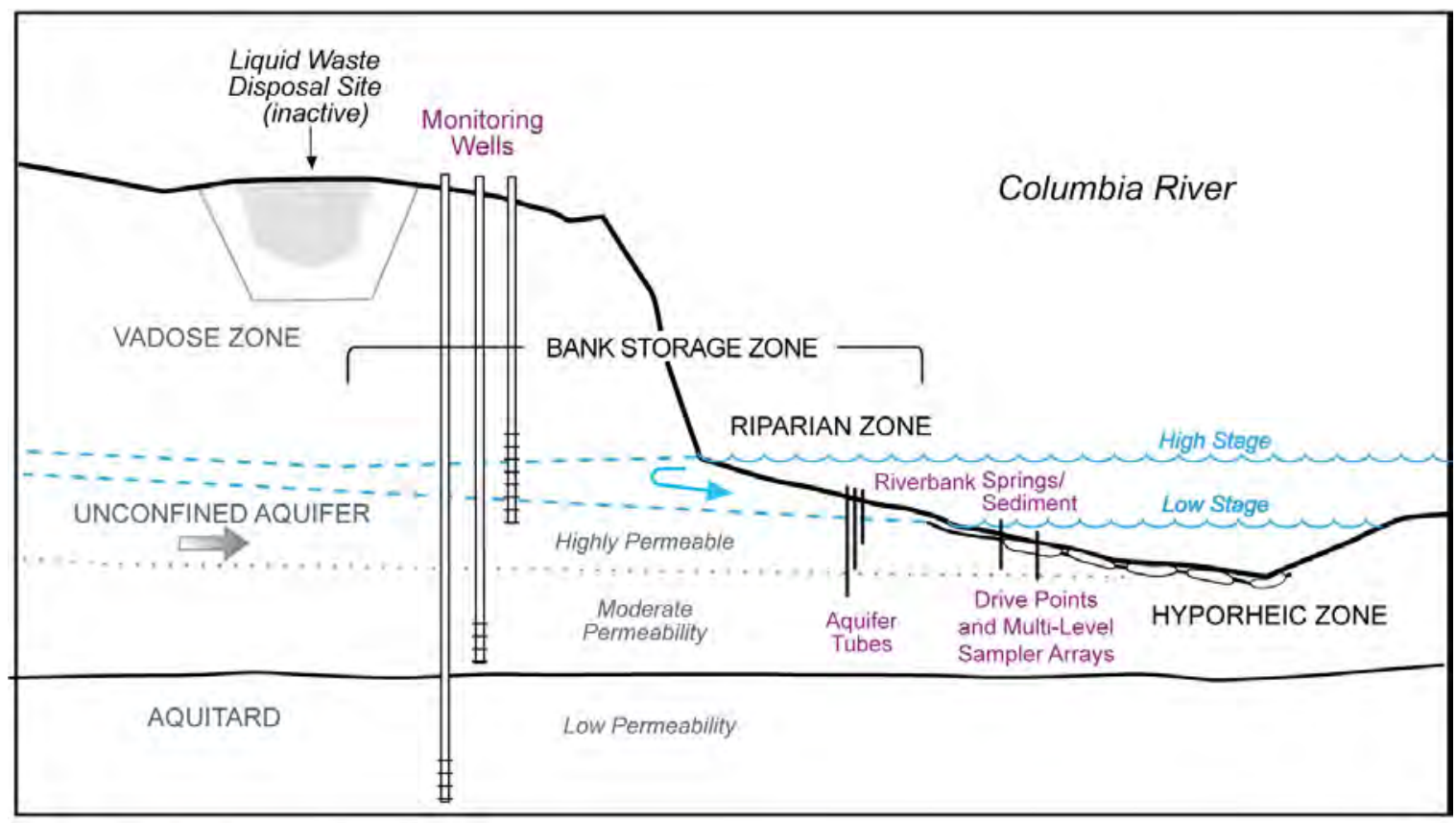

Source: PNNL-13674, Figure 1.1, with modifications

ZOI-terms-042709.ai

Figure 1.2. Illustration Showing Principal Features and Various Types of Water Quality Monitoring Sites Along the Hanford Reach

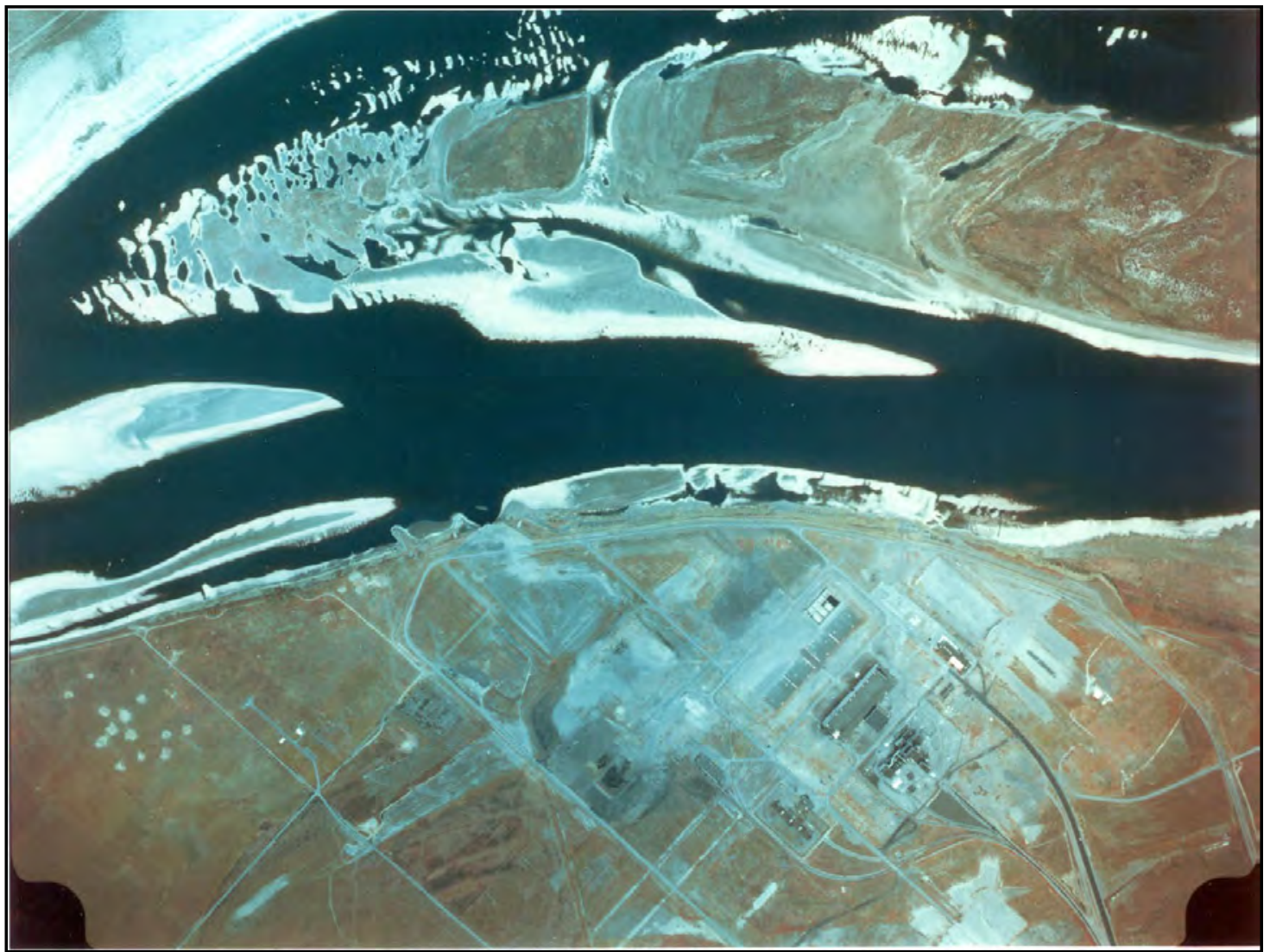

Figure 1.3. Example of 1976 Aerial Photography Showing Low River Stage Conditions (100-H Area) 
Table 1.1. Hanford River Marker (HRM) System Signposts

\begin{tabular}{|c|c|c|c|c|c|c|c|}
\hline $\begin{array}{c}\text { Hanford } \\
\text { River Marker } \\
\text { Name }\end{array}$ & Segment $^{1}$ & $\begin{array}{c}\text { Northing } \\
\text { (m-NAD83) }\end{array}$ & $\begin{array}{c}\text { Easting } \\
\text { (m-NAD83) }\end{array}$ & $\begin{array}{l}\text { Coordinate } \\
\text { Quality }^{2}\end{array}$ & $\begin{array}{c}\text { Coordinate } \\
\text { Type }^{2}\end{array}$ & $\begin{array}{l}\text { Coordinate } \\
\text { Reference }\end{array}$ & Site Description $^{3}$ \\
\hline HRM-1 & VB & $146,101.700$ & $560,218.900$ & SURV & GPS & G. Patton $(11 / 3 / 99)$ & \\
\hline HRM-2 & VB & $146,209.700$ & $561,705.100$ & SURV & GPS & G. Patton $(11 / 3 / 99)$ & In grove of vegetation, hard to see \\
\hline HRM-3 & VB & $145,727.400$ & $563,293.600$ & SURV & GPS & G. Patton $(11 / 3 / 99)$ & \\
\hline HRM-4 & BC5 & $145,306.500$ & $565,094.700$ & SURV & GPS & G. Patton $(11 / 3 / 99)$ & \\
\hline HRM-5 & BC5 & $145,835.400$ & $566,849.100$ & SURV & GPS & G. Patton $(11 / 3 / 99)$ & Near Old Irrigation Bld Between 100-B and 100-K \\
\hline HRM-6 & KR4 & $146,553.700$ & $567,972.300$ & SURV & GPS & G. Patton $(11 / 3 / 99)$ & Upriver from 100-K Launch \\
\hline HRM-7 & KR4 & $147,545.700$ & $569,208.700$ & SURV & GPS & G. Patton $(11 / 3 / 99)$ & Downriver from 100-K \\
\hline HRM-8 & KR4 & $148,576.900$ & $570,282.900$ & SURV & GPS & G. Patton $(11 / 3 / 99)$ & Upriver from 100-N \\
\hline HRM-9 & NR2 & $150,022.200$ & $571,449.800$ & SURV & GPS & G. Patton $(11 / 3 / 99)$ & Top of Bluff 100-N Springs \\
\hline HRM-10 & NR2 & $151,349.900$ & $572,435.900$ & SURV & GPS & G. Patton $(11 / 3 / 99)$ & Top of Bluff Between 100-N and 100-D \\
\hline HRM-11 & HR3D & $152,413.200$ & $573,584.900$ & SURV & GPS & G. Patton $(11 / 3 / 99)$ & Top of Bluff at 100-D Spring \\
\hline HRM-12 & HR3D & $154,033.800$ & $574,282.500$ & SURV & GPS & G. Patton $(11 / 3 / 99)$ & On floodplain \\
\hline HRM-13 & HR3D & $154,418.400$ & $575,371.700$ & SURV & GPS & G. Patton $(11 / 3 / 99)$ & Near warning siren \\
\hline HRM-14 & HR3H & $153,987.000$ & $576,716.800$ & SURV & GPS & G. Patton $(11 / 3 / 99)$ & Top of Bluff Between Locust Groves Upriver from $100-\mathrm{H}$ \\
\hline HRM-15 & HR3H & $153,164.200$ & $577,856.100$ & SURV & GPS & G. Patton $(11 / 3 / 99)$ & $\begin{array}{l}\text { Upriver of } 100-\mathrm{H} \text { Reactor (moved from island in flood plain } \\
\text { to top of bluff) }\end{array}$ \\
\hline HRM-16 & HR3H & $151,784.300$ & $579,064.900$ & SURV & GPS & G. Patton $(11 / 3 / 99)$ & Peninsula above White Bluffs Slough \\
\hline HRM-17 & $\mathrm{HR} 3 \mathrm{H}$ & $150,731.300$ & $579,959.300$ & SURV & GPS & G. Patton $(11 / 3 / 99)$ & $\begin{array}{l}\text { Peninsula above White Bluffs Slough; moved from } 150730 \\
580017\end{array}$ \\
\hline HRM-18 & FR3 & $149,036.700$ & $579,862.100$ & SURV & GPS & G. Patton $(11 / 3 / 99)$ & On floodplain, near powerline \\
\hline HRM-19 & FR3 & $147,971.200$ & $581,182.400$ & SURV & GPS & G. Patton $(11 / 3 / 99)$ & Top of Bluff, Head of 100-F Slough \\
\hline HRM-20 & FR3 & $147,269.000$ & $582,810.000$ & SURV & GPS & G. Patton $(11 / 3 / 99)$ & On Island, 100-F Slough \\
\hline HRM-21 & FR3 & $145,603.700$ & $583,401.200$ & SURV & GPS & G. Patton $(11 / 3 / 99)$ & On Island \#10, 100-F Slough \\
\hline HRM-22 & FR3 & $144,716.500$ & $582,978.000$ & SURV & GPS & G. Patton $(11 / 3 / 99)$ & \\
\hline HRM-23 & HTS & $143,317.400$ & $583,399.500$ & SURV & GPS & G. Patton $(11 / 3 / 99)$ & \\
\hline HRM-24 & HTS & $142,044.300$ & $584,340.700$ & SURV & GPS & G. Patton $(11 / 3 / 99)$ & On floodplain \\
\hline HRM-25 & HTS & $140,951.900$ & $585,444.900$ & SURV & GPS & G. Patton $(11 / 3 / 99)$ & Top of Bluff, upriver of wooden powerline \\
\hline HRM-26 & HTS & $139,191.800$ & $586,664.200$ & SURV & GPS & G. Patton $(11 / 3 / 99)$ & Downriver from Hanford Townsite Launch \\
\hline HRM-27 & HTS & $138,322.200$ & $587,875.300$ & SURV & GPS & G. Patton $(11 / 3 / 99)$ & \\
\hline HRM-28 & HTS & $137,619.300$ & $589,034.800$ & SURV & GPS & G. Patton $(11 / 3 / 99)$ & $\begin{array}{l}\text { Top of Bluff, Lower Hanford Townsite (SESP spring } \\
\text { location) }\end{array}$ \\
\hline HRM-29 & HTS & $136,638.100$ & $590,127.100$ & SURV & GPS & G. Patton $(11 / 3 / 99)$ & Top of Bluff, End of Hanford Townsite Road \\
\hline HRM-30 & PO1 & $135,399.300$ & $591,065.800$ & SURV & GPS & G. Patton $(11 / 3 / 99)$ & Top of Bluff, across from Savage Island \\
\hline
\end{tabular}


Table 1.1. (contd)

\begin{tabular}{|c|c|c|c|c|c|c|c|}
\hline \begin{tabular}{|c|} 
Hanford \\
River Marker \\
Name
\end{tabular} & Segment $^{1}$ & $\begin{array}{c}\text { Northing } \\
\text { (m-NAD83) }\end{array}$ & $\begin{array}{c}\text { Easting } \\
\text { (m-NAD83) }\end{array}$ & $\begin{array}{l}\text { Coordinate } \\
\text { Quality }^{2}\end{array}$ & $\begin{array}{c}\text { Coordinate } \\
\text { Type }^{2}\end{array}$ & $\begin{array}{l}\text { Coordinate } \\
\text { Reference }\end{array}$ & Site Description ${ }^{3}$ \\
\hline HRM-31 & PO1 & $134,043.500$ & $592,441.500$ & SURV & GPS & G. Patton $(11 / 3 / 99)$ & Below the Bluff \\
\hline HRM-32 & PO1 & $132,452.900$ & $593,866.700$ & SURV & GPS & G. Patton (11/3/99) & Top of Bluff, 1st Island above Ringold \\
\hline HRM-33 & PO1 & $130,796.060$ & $594,367.436$ & SURV & GPS & G. Patton $(11 / 3 / 99)$ & Top of Bluff, Across from top of island at Ringold \\
\hline HRM-34 & PO1 & $129,668.900$ & $594,457.600$ & SURV & GPS & G. Patton $(11 / 3 / 99)$ & Top of Bluff, Ringold Powerline \\
\hline HRM-35 & PO1 & $128,163.400$ & $594,616.200$ & SURV & GPS & G. Patton $(11 / 3 / 99)$ & On floodplain, between Powerlines \\
\hline HRM-36 & PO1 & $126,690.600$ & $594,797.200$ & SURV & GPS & G. Patton (11/3/99) & 800 m Downriver from WPPSS Pumphouse \\
\hline HRM-37 & PO1 & $125,024.100$ & $594,526.000$ & SURV & GPS & G. Patton (11/3/99) & Top of Bluff, Head of Wooded Island \\
\hline HRM-38 & PO1 & $123,584.400$ & $594,334.700$ & SURV & GPS & G. Patton $(11 / 3 / 99)$ & Top of Bluff, Lower End of Wooded Island \\
\hline HRM-39 & PO1 & $121,834.400$ & $594,411.200$ & SURV & GPS & G. Patton $(11 / 3 / 99)$ & Top of Bluff \\
\hline HRM-40 & PO1 & $120,003.300$ & $594,636.700$ & SURV & GPS & G. Patton (11/3/99) & Top of Bluff \\
\hline HRM-41 & 3FF5 & $118,206.600$ & $594,364.400$ & SURV & GPS & G. Patton (11/3/99) & Top of Bluff, 800 m Upriver from 300-TEDF \\
\hline HRM-42 & 3FF5 & $116,897.300$ & $594,340.800$ & SURV & GPS & G. Patton $(11 / 3 / 99)$ & North Side of 300 Area on Bluff \\
\hline HRM-43 & 3FF5 & $115,016.900$ & $594,765.100$ & SURV & GPS & G. Patton (11/3/99) & Between 300-Area and Unimproved Boat Launch \\
\hline HRM-44 & 3FF5 & $113,627.900$ & $594,952.300$ & SURV & GPS & G. Patton $(11 / 3 / 99)$ & Just upriver of Submarine Compartment Dock \\
\hline HRM-45 & RLD & $112,109.700$ & $595,188.600$ & SURV & GPS & G. Patton $(11 / 3 / 99)$ & Above WSU-TC, near bike path \\
\hline HRM-46 & RLD & $110,580.300$ & $595,462.000$ & SURV & GPS & G. Patton (11/3/99) & Ferry St. Richland \\
\hline \multicolumn{8}{|c|}{ Coordinate data for some of the original marker posts (i.e., pre-1999): } \\
\hline HRM-4-old & BC5 & $145,305.399$ & $565,061.998$ & SURV & GPS & IT92 (4/5/93) & \\
\hline HRM-5-old & $\mathrm{BC5}$ & $145,825.000$ & $566,865.000$ & EST & ERC-map & IT92 (4/5/93) & \\
\hline HRM-6-old & KR4 & $146,573.017$ & $567,944.824$ & SURV & GPS & IT92 (4/5/93) & \\
\hline HRM-7-old & KR4 & $147,565.000$ & $569,205.000$ & EST & ERC-map & IT92 (4/5/93) & \\
\hline HRM-8-old & KR4 & $148,537.825$ & $570,279.382$ & SURV & GPS & IT92 (4/5/93) & \\
\hline HRM-9-old & NR2 & $150,051.230$ & $571,375.296$ & SURV & GPS & IT92 (4/5/93) & \\
\hline HRM-10-old & NR2 & $151,299.185$ & $572,424.124$ & SURV & GPS & IT92 (4/5/93) & \\
\hline HRM-11-old & HR3D & $152,407.000$ & $573,573.000$ & EST & ERC-map & IT92 (4/5/93) & \\
\hline HRM-12-old & HR3D & $154,027.612$ & $574,254.963$ & SURV & GPS & IT92 (4/5/93) & \\
\hline HRM-14-old & $\mathrm{HR} 3 \mathrm{H}$ & $153,948.333$ & $576,776.371$ & SURV & GPS & IT92 (4/5/93) & \\
\hline HRM-15-old & $\mathrm{HR} 3 \mathrm{H}$ & $153,156.899$ & $577,908.022$ & SURV & GPS & IT92 (4/5/93) & \\
\hline HRM-16-old & HR3H & $151,787.098$ & $579,070.712$ & SURV & GPS & IT92 (4/5/93) & \\
\hline HRM-18-old & FR3 & $149,075.914$ & $579,844.914$ & SURV & GPS & IT92 (4/5/93) & \\
\hline HRM-19-old & FR3 & $147,937.039$ & $581,183.455$ & SURV & GPS & IT92 (4/5/93) & \\
\hline HRM-21-old & FR3 & $145,615.042$ & $583,434.102$ & SURV & GPS & IT92 (4/5/93) & \\
\hline
\end{tabular}


Table 1.1. (contd)

\begin{tabular}{|c|c|c|c|c|c|c|c|}
\hline $\begin{array}{c}\text { Hanford } \\
\text { River Marker } \\
\text { Name }\end{array}$ & Segment $^{1}$ & $\begin{array}{l}\text { Northing } \\
\text { (m-NAD83) }\end{array}$ & $\begin{array}{l}\text { Easting } \\
\text { (m-NAD83) }\end{array}$ & $\begin{array}{l}\text { Coordinate } \\
\text { Quality }^{2}\end{array}$ & $\begin{array}{c}\text { Coordinate } \\
\text { Type }^{2}\end{array}$ & $\begin{array}{l}\text { Coordinate } \\
\text { Reference }\end{array}$ & Site Description $^{3}$ \\
\hline HRM-22-old & FR3 & $144,718.908$ & $582,939.355$ & SURV & GPS & IT92 (4/5/93) & \\
\hline HRM-23-old & HTS & $143,818.114$ & $583,339.363$ & SURV & GPS & IT92 (4/5/93) & \\
\hline HRM-24-old & HTS & $142,077.695$ & $584,335.092$ & SURV & GPS & IT92 (4/5/93) & \\
\hline HRM-25-old & HTS & $140,987.831$ & $585,402.235$ & SURV & GPS & IT92 (4/5/93) & \\
\hline \multicolumn{8}{|c|}{$\begin{array}{l}1 \text { Abbreviations: VB = Vernita Bridge; BC5 = 100-B Area; KR4 = 100-K Area; NR2 = 100-N Area; HR3D = 100-D Area; HR3H = 100-H Area; FR3 = 100-F Area; HTS = Hanford Town } \\
\text { Site; PO1 = Hanford town site downstream to } 300 \text { Area; } 3 \text { FF5 = } 300 \text { Area; RLD = North Richland } \\
2 \text { EST = estimated from field narratives or geographic information system maps; SURV = surveyed using traditional or global positioning system (GPS) methods } \\
{ }^{3} \text { Verbatim from historical notebook entries }\end{array}$} \\
\hline
\end{tabular}





\subsection{Historical Investigations and Previous Work}

The exposure of contaminated groundwater along the riverbank and release of reactor coolant to the river via outfalls and spillways were significant concerns during the reactor operating years (primarily the late 1940s to mid-1960s). Intentional and accidental release to the soil column of the huge volumes of liquid effluent generated at each reactor resulted in groundwater mounds that accentuated flow toward the river, as illustrated in Figure 2.1 (Brown 1963). At some locations, steaming riverbank springs could be observed during periods of low river stage (Figure 2.2). Areas of effluent springs and the resulting thermal plume in the river were traced using infrared imagery (Eliason 1969). Additional contaminant load was presented to the shoreline environment by the operation of concrete effluent spillways at each reactor area (also shown in Figure 2.2). These spillways dispersed reactor coolant and other effluents that would normally be discharged to the center of the river channel via outfall pipelines, except when the river stage was too high for flow via the pipelines (HAPO 1963).

An aerial view of the 100-K Area during operations in 1965 is shown in Figure 2.3. Overland flow of effluent disposed to infiltration cribs and trenches is visible near some disposal sites and is responsible for the enhanced growth of vegetation (greenish areas) near the river. Because of a mound that formed above the ambient water table beneath the disposal trench at the top of the photo, riverbank springs were more prominent during the operating years than under current conditions. Residual amounts of contaminants, such as chromium, nitrate, strontium-90, tritium, and uranium, remain in the vadose zone and upper portion of the unconfined aquifer at near-river locations. These contaminants are monitored under existing programs.

Some monitoring of riverbank springs was conducted during the peak years of the cold war (i.e., 1950s and 1960s), although formal environmental monitoring did not start until the 1970s. By the late 1960s and into the early 1980s, public awareness of the potential danger created by contaminants exposed along the river shore prompted more comprehensive monitoring, which was performed by Battelle Northwest Laboratory (subsequently Pacific Northwest Laboratory and Pacific Northwest National Laboratory). Nitrate, tritium, uranium, and gross beta concentrations were typically measured for riverbank springs samples.

The following sections in this chapter describe the principal projects that have collected, or continue to collect, water quality data from sampling sites along the facilities side of the Hanford Reach shoreline. This background information is presented to provide perspective on the naming conventions previously used.

\subsection{Sitewide Environmental Surveillance Under Pacific Northwest Laboratory}

The first comprehensive survey of riverbank springs along the Hanford Site shoreline was conducted between fall 1982 and fall 1983 by the Hanford Environmental Surveillance Program (McCormack and Carlile 1984). At that time, the program's objective was to evaluate discharges to the Columbia River that occurred as the result of Hanford operations. The riverbank springs study supplemented routine monitoring activities, which focused primarily on analysis of river water. 
During 1982, the shoreline from just upstream of the 100-B Area downstream to North Richland was visually inspected. Approximately 115 locations were identified where groundwater appeared at the surface during periods of low river stage. In 1983, many of the previously identified locations were revisited and riverbank spring water was sampled. In addition, river water was collected from within several meters of the shoreline adjacent to each spring sampled. Selected samples were analyzed for nitrate, gross beta, iodine-129, strontium-90, technetium-99, tritium, and uranium. Because the specific conductance of the samples was not measured, estimates for the relative proportions of groundwater and river water in the spring samples are not available.

The numbering system for the 1982-1983 springs investigation used HRM signposts, which subdivide the shoreline between Vernita Bridge and North Richland into 46 segments, each approximately one-mile long. ${ }^{1}$ A spring site name was derived using the nearest upstream marker, to which a suffix was added that indicated the sequence of the spring site downstream of the marker. For example, spring site " $3-5$ " indicated the fifth spring observed downstream from HRM number 3.

A second comprehensive survey of riverbank springs was completed by the PNL environmental surveillance project during 1988 (Dirkes 1990). This study provided a follow-up to the initial 1982-1983 riverbank springs study, with an expanded geographic coverage and a more extensive suite of analyses. Near-shore river water samples were collected from locations adjacent to where riverbank springs were sampled. Samples were also collected from sites in Franklin County across the river from Hanford facilities and from irrigation return canals. An important discovery was that some radiological constituents that are typical of Hanford Site groundwater (e.g., uranium and the associated gross alpha activity) were present in irrigation return water at levels higher than in Columbia River water.

The location names used for riverbank springs during the 1988 survey followed the convention established by the earlier 1982 survey. However, the analytical results were tabulated according to HRM position and were not given discreet location names (Dirkes 1990). As for the earlier survey, specific conductance measurements for the samples were not reported.

\subsection{Environmental Restoration Under Westinghouse Hanford Company}

A third comprehensive survey of riverbank springs was conducted in fall 1991 as part of environmental restoration project activities (DOE 1992). The work was prompted by an increased need for information to be used by DOE and regulatory agencies to support decisions regarding groundwater contamination near the river. A Tri-Party Agreement (Ecology 1989) major milestone was framed to provide impetus for generating this information (major milestone M-30; sub-milestone M-30-01).

The PNL environmental surveillance project provided considerable help in planning the fall 1991 environmental restoration project. PNL staff outlined all known areas along the shoreline, where springs had been observed previously, on the newly-acquired 1:2,000 series of topographic maps for the Hanford

\footnotetext{
${ }^{1}$ No published reference to the initial installation of these markers has been identified. They were subsequently reset and surveyed during summer 1999 by the Surface Environmental Surveillance Project (SESP), successor to the original surveillance program.
} 
Site. ${ }^{2}$ A new naming system for riverbank spring locations was devised, again based on the HRM signposts, and efforts were made to associate all previously sampled locations with the new naming convention. The new format consisted of the location to the nearest tenth of a HRM signpost, followed by a suffix used to indicate multiple springs at the same approximate location. For example, springs 057$1,057-2$, etc. would indicate multiple springs at a site located seven tenths of the downstream distance between HRM signposts \#5 and \#6 (i.e., HRM 5.7).

During this project, samples were collected of (1) riverbank spring water, (2) fine-grained sediment associated with springs, and (3) nearshore river water adjacent to springs. Twenty-six locations were sampled; analyses included a comprehensive suite of chemical and radiological contamination indicators. The sampling and analysis activities were completed following CERCLA protocols for obtaining environmental data to support restoration decisions. Data to estimate the relative proportions of groundwater and river water in the spring samples were collected by recording the specific conductance for each sample and for nearshore river water, along with temperature and $\mathrm{pH}$. As a consequence, it became possible to discuss the results not only in terms of concentrations at the location and time of exposure at the spring, but also to make a qualitative statement regarding how representative the concentrations were of nearby groundwater. The data collected during this project are stored in the HEIS under the surface water (SW) media category.

The data collected during fall 1991 were further analyzed to better understand the relationship between water quality data obtained from near-river wells, riverbank springs, and near-shore river water. Concentrations of various contamination indicators were plotted as function of position along the shoreline. The results showed a good correlation with previously mapped groundwater plumes, and also that concentrations in exposed riverbank springs tended to fall in a range between values in groundwater and adjacent river water, although considerable variability existed. Additional work to provide consistent names, HRM references, and geographic coordinates was completed during this time period. The results were published in an interpretive report (Peterson and Johnson 1992). The principal conclusions presented in that report are:

- Riverbank spring water concentrations for contamination indicators typically fall within the range bounded by groundwater (high) and nearshore river water (low) concentrations. Within that range, spring water concentrations vary in response to river stage fluctuations.

- The timing of sample collection relative to river stage characteristics prior to sampling must be considered when determining the representativeness of the sample. The height and duration of river stage cycles (daily and seasonal) are key factors that influence water quality at riverbank springs.

- Data from riverbank springs samples are not the most representative data to use for estimating groundwater contaminant flux to the Columbia River.

The environmental restoration program resampled the 1991 riverbank springs during 1993. There are no published reports that describe the results of this sampling. Many of these sites were then incorporated into the CERCLA remedial investigation sampling and analysis schedules associated with the 100 Area groundwater Operable Units.

\footnotetext{
${ }^{2}$ Personal communication to Westinghouse Hanford Company staff from RL Dirkes, Pacific Northwest Laboratory, Richland, Washington, dated 1991.
} 
Shoreline sampling and analysis was also conducted at the 300 Area as part of the limited field investigation for the 300-FF-5 groundwater Operable Unit (Friant and Hulstrom 1993). In that investigation, near-river wells, riverbank springs, shoreline sediments, and near-shore river water samples were collected during September 1992. The samples were analyzed for radionuclides, metals, and organic constituents. Uranium was identified as the chief contaminant of concern, with the primary source being nuclear fuel fabrication effluent that was discharged to the 300 Area Process Trenches, and earlier to the North and South Process Ponds, and the 307 Process Trenches. The investigation also concluded that shoreline sediment did not appear to be an important sink for contaminants.

\subsection{Environmental Restoration Under Bechtel Hanford, Inc.}

The Environmental Restoration Contractor team, initially involving Bechtel Hanford, Inc., CH2M HILL Hanford Inc., and IT Hanford, Inc., assumed responsibility for the environmental restoration program in July 1994. This responsibility included follow-on work to TPA Milestone M-30-05 that involved the groundwater/river interface. Several projects provided new and unique data from the interface in the 100 Area. Riverbank springs sampling as part of the environmental restoration program was conducted each fall since 1996. Sampling logistics were integrated with shoreline monitoring activities conducted under the Surface Environmental Surveillance Project (SESP), which is run by Pacific Northwest National Laboratory (PNNL).

During spring 1995, CH2M HILL divers collected samples of substrate pore water from the riverbed adjacent to the 100-H Area (Hope and Peterson 1996a; Figure 2.4). The area for the investigation included riverbed substrate that is heavily used by fall Chinook salmon as spawning habitat. The area is also adjacent to where groundwater contaminated by chromium enters the river. The pore water samples were analyzed for hexavalent chromium using several methods, including adsorptive stripping voltametry, which offers very low detection levels (Olsen and Kucheryavyy 1995). At one location within a gravelly area used as a redd (collection of egg pockets), chromium was observed at concentrations up to $130 \mu \mathrm{g} / \mathrm{L}$, which is well above the federal standard for protecting freshwater aquatic organisms (i.e., $11 \mu \mathrm{g} / \mathrm{L}$ ). The specific conductance of the sample indicated that it contained a significant proportion of river water, so the concentration of groundwater approaching that area might have been even higher than the measured value.

A second field investigation using divers to collect pore water samples from the riverbed was conducted during fall 1995 at the 100-D Area (Hope and Peterson 1996b; Figure 2.5). In addition to collecting pore water samples, numerous sampling tubes fitted with 6-inch long stainless steel screens were installed in the aquifer at multiple depths along the shoreline. Along one segment of the shoreline, samples contained hexavalent chromium at concentrations higher than previously observed in any other water samples from the 100 Area. The highest concentrations observed were $630 \mu \mathrm{g} / \mathrm{L}$ in pore water from riverbed sediment and $870 \mu \mathrm{g} / \mathrm{L}$ in an aquifer tube at the shoreline adjacent to the pore water site. This discovery led to the installation of new groundwater monitoring wells and vigorous investigation of potential sources, including a reconstruction of the water table that may have existed during the operating years (Connelly 1997). The newly discovered area of chromium contamination became known as the 100-D "hot spot." The plume was initially treated using an in situ redox treatment system to form a reactive barrier that prevents or reduces the mass flux of chromium into the river (Williams et al. 2000). 
The following year (1996), divers returned to pore water sampling sites at 100-H, 100-D, and 100-K Areas and installed permanent pore water sampling ports, with tubing leading ashore to a point above the high-water line. ${ }^{3}$ These tubes, along with additional permanent installations at 100-D and 100-K Areas, may still be viable for sample collection.

An additional method for shoreline monitoring was developed as part of the riverbed pore water sampling investigation. This method involved using direct-push tools to implant small diameter tubes at multiple depths in the aquifer beneath the shoreline (subsequently referred to as aquifer sampling tubes). Based on the success of this method at the 100-D Area, a more comprehensive installation project was developed, which resulted in nearly continuous coverage of the shoreline from the 100-B Area downstream to the Hanford town site, at intervals of $\sim 600$ meters ( 2,000 feet) (Peterson et al. 1998).

In fall 2002, work scope involving aquifer sampling tubes was transferred from the environmental restoration contractor to PNNL, where it became incorporated as part of the groundwater project. Subsets of these tubes were subsequently resampled annually by PNNL to collect data that support environmental restoration program objectives (e.g., Hartman and Peterson 2003). Sampling of the network continued at PNNL until being transferred to Fluor Hanford, Inc. in 2006.

\subsection{Soil and Groundwater Remediation Under Fluor Hanford, Inc. and CH2M Hill Plateau Remediation Company}

Starting in 2003, the aquifer tube network was enhanced by additional installations at areas not initially covered and to replace damaged or lost tubes. A summary of the several expansion campaigns is provided in the updated sampling and analysis plan for the network (Kasza 2009). An evaluation of how to optimize use of the tube network was recently completed that includes a comprehensive description of the network, along with summaries of recent analytical results for constituents of interest and graphics that illustrate the positions of tube and near-river well screens to stratigraphic intervals beneath the shoreline (Hartman 2009).

\subsection{River Corridor Baseline Risk Assessment Under Washington Closure Hanford, LLC}

The River Corridor Baseline Risk Assessment (RCBRA) project began in 2004 with an extensive effort toward identifying and compiling all available environmental data for various segments of the Columbia River (DOE-RL 2008b). The project is still underway and involves comprehensive sampling of the river environment where it crosses the Hanford Site. During 2008 and 2009, numerous samples of pore water were collected from approximately a 10-inch depth in the riverbed sediment, using newly developed field methods and equipment to accommodate the dynamic conditions encountered in the Columbia River. Considerable effort was expended to bias sampling towards obtaining samples that were representative of groundwater, i.e., when discharge of groundwater to the river was at its maximum rate. This sampling effort is producing significant new information on the discharge of groundwater and

\footnotetext{
${ }^{3}$ Interoffice Memorandum Control No. 044041, from SJ Hope (CH2M Hill Hanford, Inc.) to MH Sturges and AJ Knepp (Bechtel Hanford, Inc.), Installation of Riverbed Pore Water Sampling Ports in the Columbia River at 100D/DR, 100-H, and 100-K Reactor Areas, dated March 11, 1997.
} 
dissolved contaminants, information that will assist in developing remedial action decisions during the next several years. The first formal reports on the locations sampled and analytical results are expected during 2010.

\subsection{Miscellaneous Investigations}

During 1988, investigators from SEARCH Technical Services, Davenport, Washington, collected samples of riverbank springs and shoreline sediment at various locations along the Hanford Reach (Buske and Josephson 1989). Their work focused on gamma-emitting radionuclides in the shoreline environment and they used the earlier work by McCormack and Carlile (1984) as a guide to sampling locations. Because they recorded their sampling locations relative to the HRM signposts, it has been possible to relate their sampling sites to those of other investigators.

As part of research into the zone of interaction between groundwater and river water, sampling methods similar to the aquifer sampling tubes just described have been employed at the $100-\mathrm{N}$ and 300 Areas starting in 2004. The objectives are to investigate the water quality characteristics in riverbed sediment that is used as habitat (i.e., the upper $\sim 0.5$ meter) and also to further refine the conceptual model that describes the dynamics of interaction. The investigations at the 300 Area are described in Fritz et al. (2009, 2008, and 2007) and Arntzen et al. (2006).

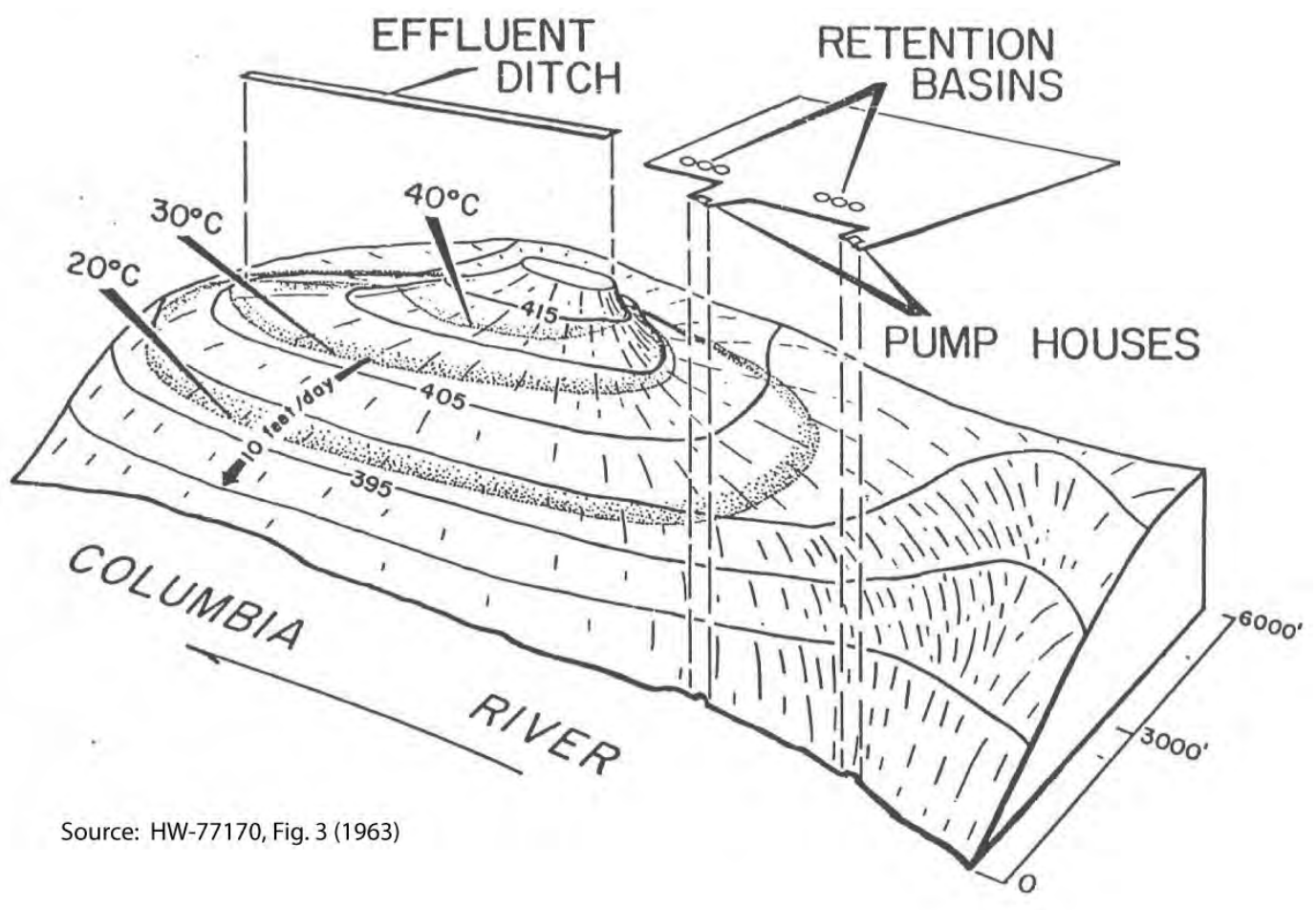

Figure 2.1. Illustration Showing Mounding of Water Table at $100-\mathrm{K}$ Area During Operations 


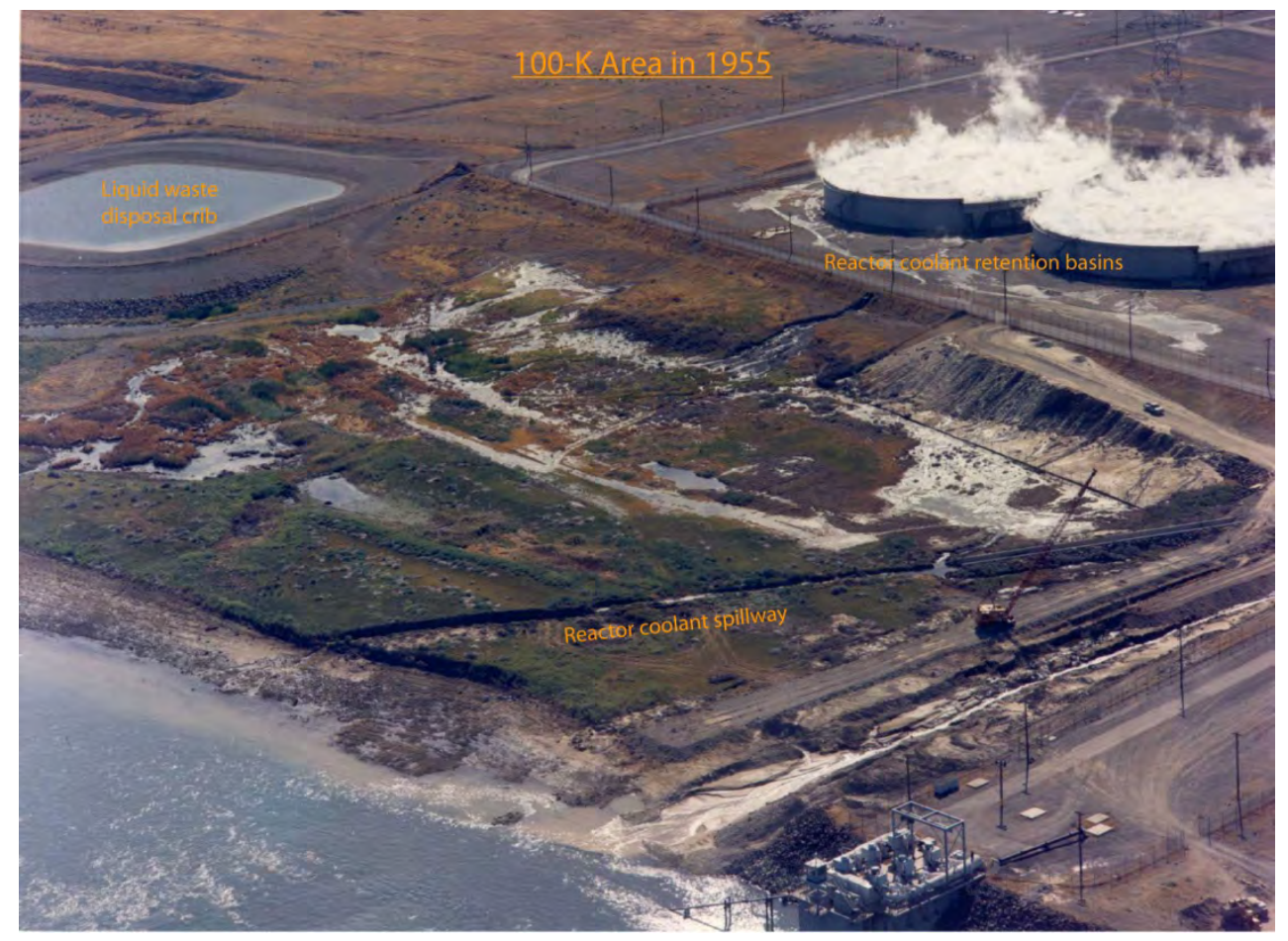

Figure 2.2. Aerial Photograph Showing Surface Runoff Across Shoreline at 100-K Area During Operations in 1955

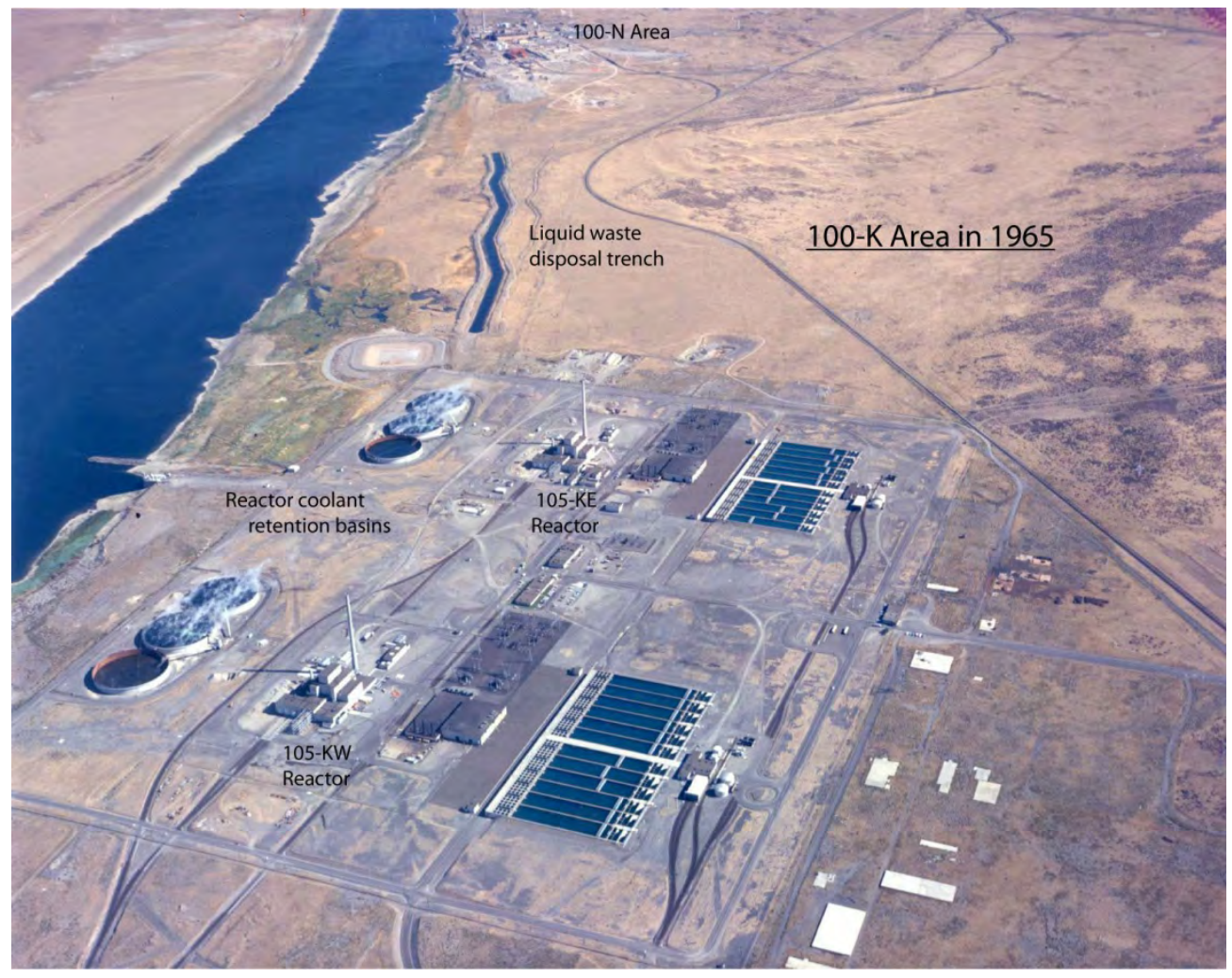

Figure 2.3. Aerial Photograph Showing 100-K Area During Operations in 1965 


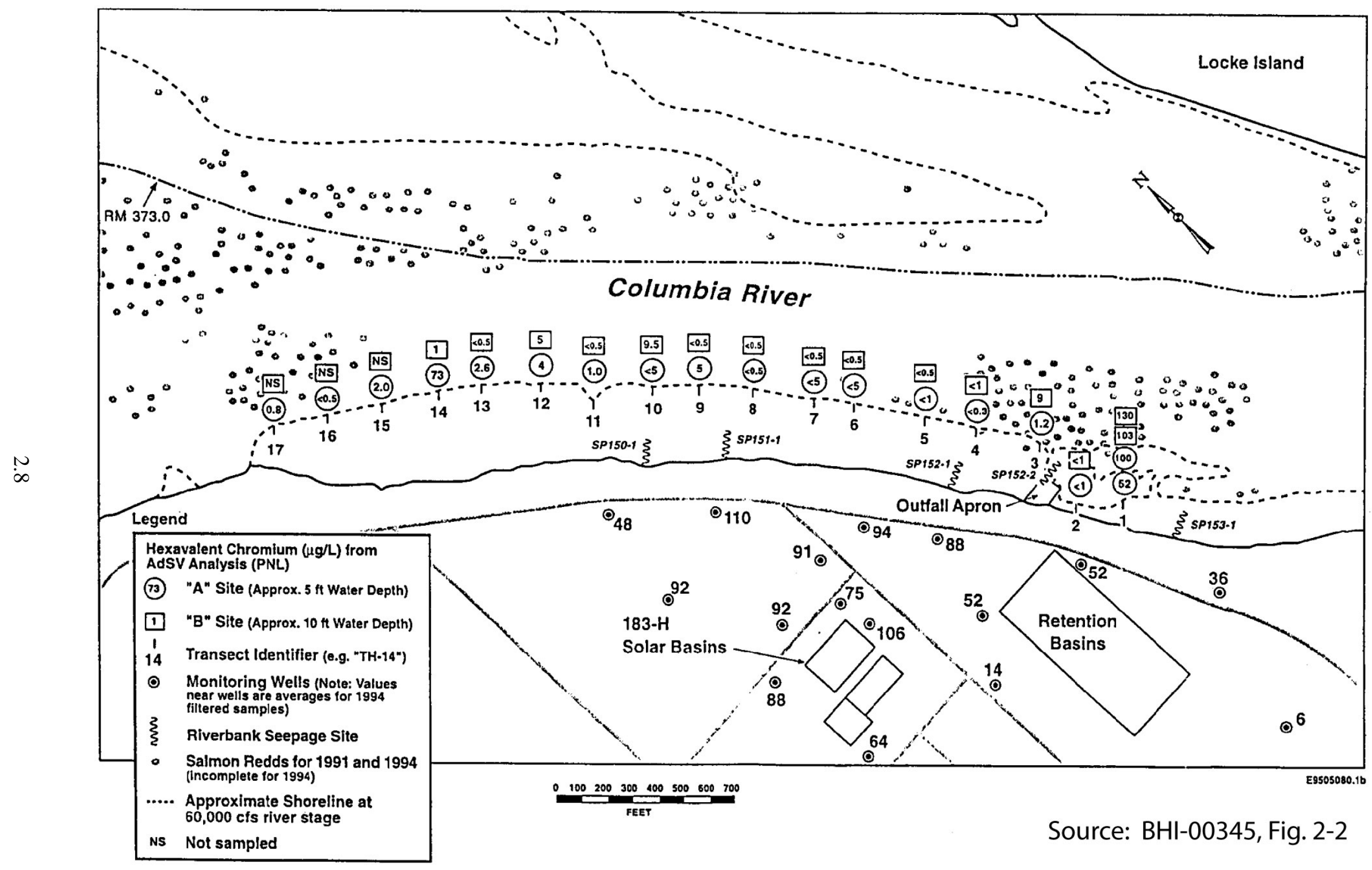

Figure 2.4. Locations Along 100-H Area Where Riverbed Pore Water Samples were Collected in March 1995 


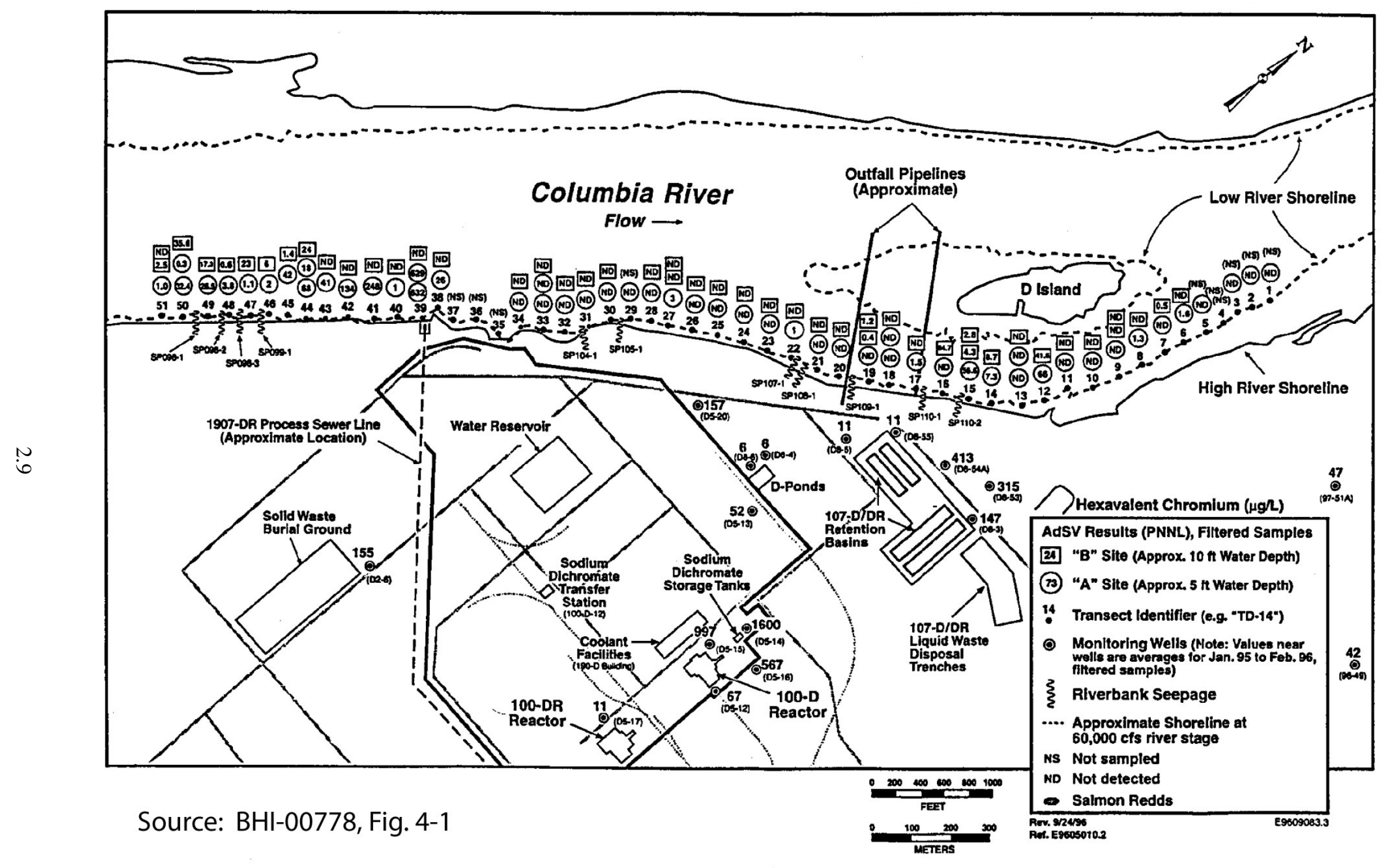

Figure 2.5. Locations Along 100-D Area Where Riverbed Pore Water Samples were Collected in October/November 1995 



\subsection{Aquifer Sampling Tubes}

An aquifer sampling tube is a small-diameter polyethylene tube inserted into the aquifer near the lowriver stage shoreline using direct-push methods. A stainless mesh screen 6 inches (15.2 centimeters) in length is attached at the lower end of the tube as a sampling port. Multiple tubes are installed at several depths at each location. Each tube is considered a separate entity for recordkeeping purposes. They provide samples of groundwater from locations as close as logistically practical to the point where groundwater discharges into the riverbed substrate.

At some sites, up to three tubes were bundled together and installed using a single temporary driven casing. At other sites, individual tubes were installed using individual temporary driven casings, which may be separated by up to several meters distance (Figure 3.1). For installations made after 2003, each tube was installed using a separately driven temporary casing (i.e., bundling was no longer used). The temporary casings were driven into the ground using either a hand-held air hammer or a truck-mounted hydraulic ram.

\subsection{Aquifer Sampling Tube Naming Conventions}

Three different conventions have been used to name the sites where aquifer sampling tubes are installed. The first convention was used during the riverbed pore water sampling investigations conducted at 100-D Area in fall 1995 (Hope and Peterson 1996b). An example for an aquifer sampling tube location for those investigations is: "DD-17-2." The first letter indicates a sampling tube that is driven into the aquifer. The second letter refers to the 100 -D Area. The number " 17 ” refers to pore water transect 17 (see Chapter 5.0 that follows for a description of "transects") and the number "2" indicates the depth of the sampling port, with " 1 " being the most shallow and " 4 " being the deepest.

The second naming convention was established for a fall 1997 project to install aquifer sampling tubes along the Hanford Reach shoreline from the 100-B Area downstream to the Hanford town site (Peterson et al. 1998). Temporary location names were created that represent the sequence of locations, progressing downstream, and a suffix that indicated the relative depth of the sampling port. For example, location "14-D" is the fourteenth location downstream of the upstream starting point. The suffix "D" indicates the deepest port in the uppermost hydrologic unit at that location ("S" stands for the shallowest port, and " $\mathrm{M}$ ” for the mid-depth port). The objective for the installations included one port approximately 1.5 meters ( 5 feet) below the water table; one port as close to the bottom of the aquifer as possible; and a third port at mid-depth between the other two.

The third convention appeared during planning for installing tubes at additional sites to fill in coverage gaps, and to replace existing tubes that no longer yielded samples. This project was conducted between December 2003 and April 2004. The names used started with a prefix “AT-” for aquifer tube, followed by a letter that designated a reactor area or shoreline segment, and ending with a sequential number. A suffix was also added to indicate the relative depth of each individual tube at a site. For example, AT-B-5-M is an aquifer sampling tube at the 100-B Area. It is the fifth site at 100-B equipped during the installation campaign in 2004 and is the medium-depth tube.

Because the aquifer sampling tubes are considered "resource protection wells" under the Washington State Administrative Code (WAC 173-160), they have been assigned well identification numbers in the 
Hanford Well Information System (HWIS). These numbers serve an internal data management purpose and are not normally shown in reports.

\subsection{Aquifer Sampling Tube Location Names and Coordinates}

A listing of all aquifer sampling tubes installed under the Environmental Restoration (ER) program to support CERCLA investigations is presented in Table 3.1. Also, installations of similar facilities, variously described as piezometers and drive points, are included on the table, although no claim is made for completeness for non-ER program installations. For convenience, any small diameter (i.e., less than 5-centimeter [2-inch] diameter) tube driven into the shoreline and riverbed sediment will be referred to as an "aquifer sampling tube," a label which may indicate measurement of hydrostatic pressures and in situ monitoring equipment, as well as actual water sample collection. The columns in Table 3.1 are defined as follows:

- Well Identifier (HWIS) - Formal identifier code assigned to each groundwater monitoring well and aquifer sampling tube by the organization responsible for administering groundwater monitoring facilities on the Hanford Site. The code is used for internal data management purposes.

- Port Depth - Depth below ground surface (in feet) to the top of the six-inch screened sampling port. Typically measured by inserting a fiberglass "fish tape" down the tube; the tape "bottoms" at the top of the screen.

- Aquifer Tube Name - The name in use during 2009 for tubes routinely sampled under the CERCLA program. For the most recently installed tubes, the HWIS well identifier is being used as the descriptive name.

- HRM for Site (“2k”) - Aquifer sampling tube location relative to the Hanford River Marker system, as updated in 1999.

- Segment - Segments defined for convenience in grouping monitoring sites by CERCLA groundwater operable units, groundwater interest areas, or other common characteristic.

- Northing and Easting - Location coordinates in Washington State Plane (South Zone) system; units are meters; North American Datum of 1983.

- Coordinate Quality - Quality of the coordinates, e.g., “surveyed” using global positioning system (GPS) equipment or traditional land surveying methods; "estimated” using geographic information system (GIS) maps.

- Coordinate Reference - Citation for information on the aquifer sampling tube site.

- Ground Elevation - Vertical reference for ground surface; units are meters; North American Vertical Datum of 1988.

- Elevation Quality - Quality of the vertical reference; estimated from maps; if blank, quality is uncertain.

\subsection{Previous Names for Aquifer Tubes}

For most aquifer tube sites, the descriptive names assigned during the planning operations have not been subsequently changed. Exceptions include the tubes installed as part of the in situ Redox 
manipulation test to create a barrier to hexavalent chromium migration. Also, for a brief period during approximately 2001, attempts were made to assign names to tubes based on the convention used for naming monitoring wells (Chamness and Merz 1993), but this convention was never fully implemented. Table 3.2 lists the various names used previously to refer to some of the aquifer sampling tubes. Some column headings are the same as those used in Table 3.1, with the following exceptions:

- Name as Used in Reference - Name as used in historical report or other reference that includes aquifer sampling tube names.

- Name Reference - Report citation or other source.

- Year of Name Use - Year in which a tube name was published in the reference cited.

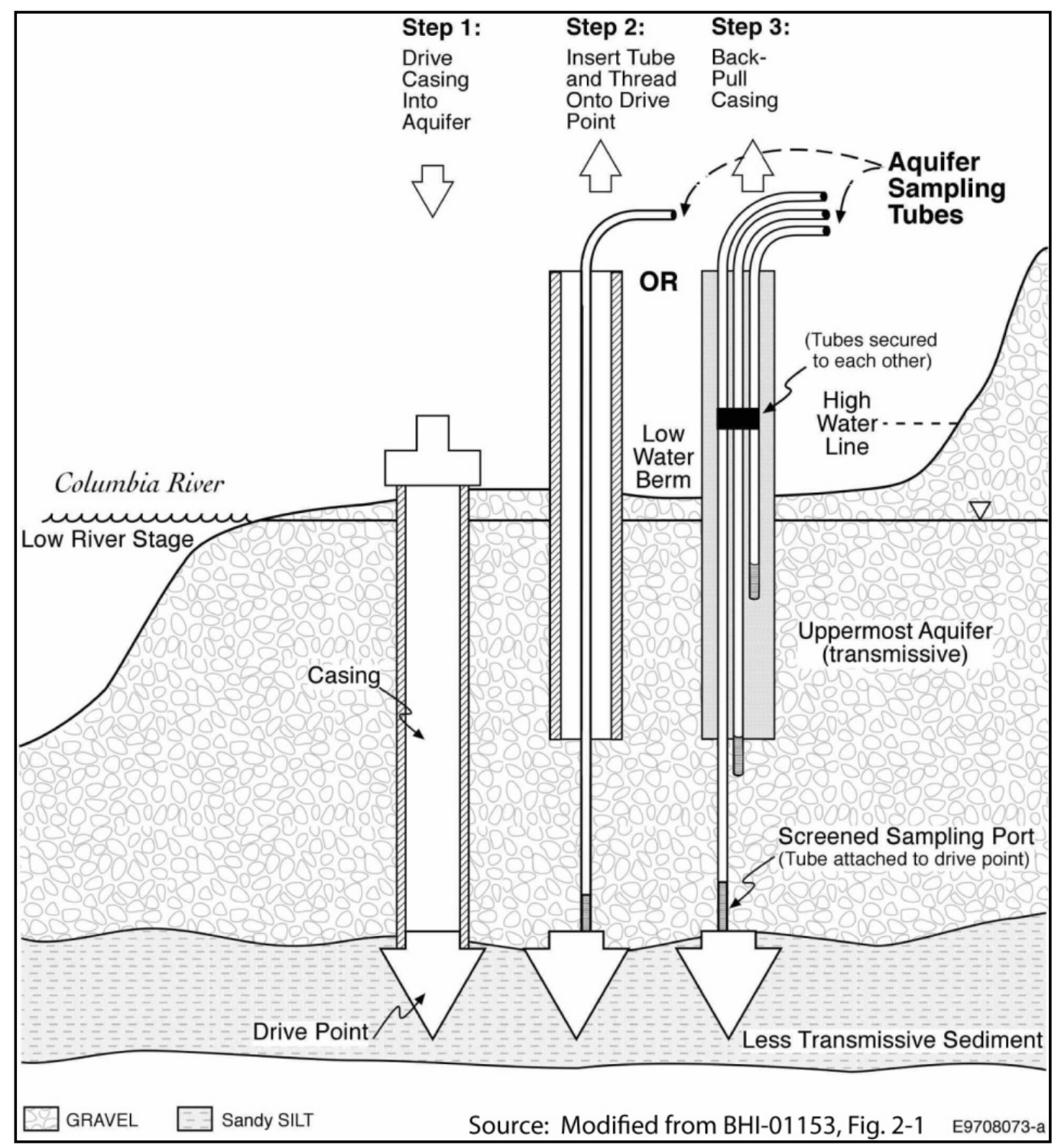

Figure 3.1. Illustration Showing Installation of Aquifer Sampling Tubes 
Table 3.1. Aquifer Sampling Tube Sites Along the Hanford Site Shoreline

\begin{tabular}{|c|c|c|c|c|c|c|c|c|c|c|}
\hline $\begin{array}{c}\text { Well } \\
\text { Identifier } \\
\text { (HWIS) }\end{array}$ & $\begin{array}{c}\text { Port } \\
\text { Depth } \\
\text { [to top of } \\
\text { opening] } \\
\text { (ft-bgs) }\end{array}$ & $\begin{array}{c}\text { Aquifer Tube } \\
\text { Name } \\
\text { (use in 2009) }\end{array}$ & $\begin{array}{l}\text { HRM } \\
\text { for Site } \\
\text { ("2k") }\end{array}$ & Segment $^{1}$ & $\begin{array}{c}\text { Northing } \\
\text { (m-NAD83) }\end{array}$ & $\begin{array}{c}\text { Easting } \\
\text { (m-NAD83) }\end{array}$ & $\begin{array}{c}\text { Coordinate } \\
\text { Quality }^{2}\end{array}$ & Coordinate Reference & $\begin{array}{c}\text { Ground } \\
\text { Elevation } \\
\text { (m-NAVD88) }\end{array}$ & $\begin{array}{c}\text { Elevation } \\
\text { Quality }^{2}\end{array}$ \\
\hline B8115 & 7.0 & $01-\mathrm{S}$ & 2.600 & VB & $146,033.078$ & $562,697.662$ & SURV & Confirmed 9/24/02 PNNL sub-meter GPS & 122.676 & \\
\hline B8114 & 16.0 & 01-M & 2.600 & VB & $146,033.078$ & $562,697.662$ & SURV & Confirmed 9/24/02 PNNL sub-meter GPS & 122.676 & \\
\hline B8113 & 24.0 & 01-D & 2.600 & VB & $146,033.078$ & $562,697.662$ & SURV & Confirmed 9/24/02 PNNL sub-meter GPS & 122.676 & \\
\hline B8118 & 6.0 & $02-S$ & 3.130 & VB & $145,689.247$ & $563,539.581$ & SURV & Confirmed 9/24/02 PNNL sub-meter GPS & 122.267 & \\
\hline B8117 & 14.9 & 02-M & 3.130 & VB & $145,689.247$ & $563,539.581$ & SURV & Confirmed 9/24/02 PNNL sub-meter GPS & 122.267 & \\
\hline B8120 & 7.0 & 03-M & 3.450 & $\mathrm{BC5}$ & $145,469.247$ & $564,077.431$ & SURV & Confirmed 9/24/02 PNNL sub-meter GPS & 121.154 & \\
\hline $\mathrm{B} 8119$ & 13.0 & 03-D & 3.450 & BC5 & $145,469.247$ & $564,077.431$ & SURV & Confirmed 9/24/02 PNNL sub-meter GPS & 121.154 & \\
\hline C4376 & 8.0 & AT-B-1-S & 3.570 & $\mathrm{BC5}$ & $145,379.276$ & $564,307.002$ & SURV & GPS ID1544 FHI-TimJohnson-March04 & 121.239 & \\
\hline C4375 & 13.3 & AT-B-1-M & 3.570 & BC5 & $145,379.938$ & $564,308.894$ & SURV & GPS ID1543 FHI-TimJohnson-March04 & 121.271 & \\
\hline C4378 & 8.6 & AT-B-2-S & 3.660 & BC5 & $145,329.643$ & $564,514.817$ & SURV & GPS ID1550 FHI-TimJohnson-March04 & 120.902 & \\
\hline C4379 & 14.0 & AT-B-2-M & 3.660 & BC5 & $145,329.366$ & $564,515.900$ & SURV & GPS ID1548 FHI-TimJohnson-March04 & 120.862 & \\
\hline $\mathrm{C} 4377$ & 19.0 & AT-B-2-D & 3.660 & $\mathrm{BC5}$ & $145,329.139$ & $564,513.954$ & SURV & GPS ID1549 FHI-TimJohnson-March04 & 120.920 & \\
\hline B8124 & 8.3 & 04-S & 3.730 & $\mathrm{BC5}$ & $145,283.419$ & $564,612.459$ & SURV & Confirmed 9/24/02 PNNL sub-meter GPS & 122.407 & \\
\hline B8123 & 13.0 & 04-M & 3.730 & BC5 & $145,283.047$ & $564,612.066$ & SURV & Confirmed 9/24/02 PNNL sub-meter GPS & 122.491 & \\
\hline $\mathrm{B} 8122$ & 25.0 & 04-D & 3.730 & $\mathrm{BC5}$ & $145,283.275$ & $564,612.184$ & SURV & Confirmed 9/24/02 PNNL sub-meter GPS & 122.484 & \\
\hline $\mathrm{C} 6227$ & 11.2 & $\mathrm{C} 6227$ & 3.810 & $\mathrm{BC5}$ & $145,303.590$ & $564,749.630$ & SURV & AST Survey Data 1995-2008-MH-07509.xls & 119.930 & \\
\hline C6228 & 17.5 & C6228 & 3.810 & $\mathrm{BC5}$ & $145,303.780$ & $564,746.340$ & SURV & AST Survey Data 1995-2008-MH-07509.xls & 119.960 & \\
\hline C6229 & 23.4 & C6229 & 3.810 & $\mathrm{BC5}$ & $145,303.790$ & $564,748.320$ & SURV & AST Survey Data 1995-2008-MH-07509.xls & 120.000 & \\
\hline B8127 & 8.5 & 05-S & 3.890 & BC5 & $145,332.290$ & $564,908.310$ & SURV & Confirmed 9/24/02 PNNL sub-meter GPS & 121.992 & \\
\hline B8126 & 17.0 & 05-M & 3.890 & $\mathrm{BC} 5$ & $145,332.290$ & $564,908.310$ & SURV & Confirmed 9/24/02 PNNL sub-meter GPS & 121.992 & \\
\hline B8125 & 25.5 & 05-D & 3.890 & BC5 & $145,332.290$ & $564,908.310$ & SURV & Confirmed 9/24/02 PNNL sub-meter GPS & 121.992 & \\
\hline C4382 & 8.1 & AT-B-3-S & 4.020 & BC5 & $145,397.266$ & $565,130.482$ & SURV & GPS ID1546 FHI-TimJohnson-March04 & 120.737 & \\
\hline $\mathrm{C} 4381$ & 14.2 & AT-B-3-M & 4.020 & $\mathrm{BC} 5$ & $145,397.704$ & $565,128.625$ & SURV & GPS ID1547 FHI-TimJohnson-March04 & 120.505 & \\
\hline $\mathrm{C} 4380$ & 23.2 & AT-B-3-D & 4.020 & BC5 & $145,397.211$ & $565,127.529$ & SURV & GPS ID1545 FHI-TimJohnson-March04 & 120.623 & \\
\hline B8130 & 8.8 & $06-S$ & 4.120 & BC5 & $145,412.052$ & $565,293.927$ & SURV & Confirmed 9/24/02 PNNL sub-meter GPS & 122.122 & \\
\hline B8129 & 15.5 & 06-M & 4.120 & $\mathrm{BC5}$ & $145,412.052$ & $565,293.927$ & SURV & Confirmed 9/24/02 PNNL sub-meter GPS & 122.122 & \\
\hline B8128 & 23.0 & 06-D & 4.120 & BC5 & $145,412.052$ & $565,293.927$ & SURV & Confirmed 9/24/02 PNNL sub-meter GPS & 122.122 & \\
\hline $\mathrm{C} 6230$ & 9.2 & C6230 & 4.200 & $\mathrm{BC} 5$ & $145,459.990$ & $565,423.770$ & SURV & AST Survey Data 1995-2008-MH-07509.xls & 119.840 & \\
\hline C6231 & 13.0 & C6231 & 4.200 & BC5 & $145,460.440$ & $565,426.020$ & SURV & AST Survey Data 1995-2008-MH-07509.xls & 119.720 & \\
\hline C6232 & 26.5 & C6232 & 4.200 & BC5 & $145,453.670$ & $565,426.350$ & SURV & AST Survey Data 1995-2008-MH-07509.xls & 120.090 & \\
\hline $\mathrm{B} 8132$ & 8.0 & $07-\mathrm{M}$ & 4.270 & BC5 & $145,495.641$ & $565,566.011$ & SURV & Confirmed 9/24/02 PNNL sub-meter GPS & 120.653 & \\
\hline B8131 & 20.0 & 07-D & 4.270 & $\mathrm{BC5}$ & $145,493.904$ & $565,564.591$ & SURV & Confirmed 9/24/02 PNNL sub-meter GPS & 120.662 & \\
\hline C6233 & 9.6 & C6233 & 4.290 & $\mathrm{BC} 5$ & $145,496.480$ & $565,561.410$ & SURV & AST Survey Data 1995-2008-MH-07509.xls & 120.230 & \\
\hline C6234 & 15.3 & C6234 & 4.290 & $\mathrm{BC5}$ & $145,495.820$ & $565,560.410$ & SURV & AST Survey Data 1995-2008-MH-07509.xls & 120.170 & \\
\hline $\mathrm{C} 6235$ & 19.2 & C6235 & 4.290 & BC5 & $145,495.240$ & $565,563.420$ & SURV & AST Survey Data 1995-2008-MH-07509.xls & 120.310 & \\
\hline
\end{tabular}


Table 3.1. (contd)

\begin{tabular}{|c|c|c|c|c|c|c|c|c|c|c|}
\hline $\begin{array}{c}\text { Well } \\
\text { Identifier } \\
\text { (HWIS) }\end{array}$ & $\begin{array}{c}\text { Port } \\
\text { Depth } \\
\text { [to top of } \\
\text { opening] } \\
\text { (ft-bgs) }\end{array}$ & $\begin{array}{l}\text { Aquifer Tube } \\
\quad \text { Name } \\
\text { (use in 2009) }\end{array}$ & $\begin{array}{c}\text { HRM } \\
\text { for Site } \\
\text { ("2k") }\end{array}$ & Segment $^{1}$ & $\begin{array}{c}\text { Northing } \\
\text { (m-NAD83) }\end{array}$ & $\begin{array}{c}\text { Easting } \\
\text { (m-NAD83) }\end{array}$ & $\begin{array}{c}\text { Coordinate } \\
\text { Quality }^{2}\end{array}$ & Coordinate Reference & $\begin{array}{c}\text { Ground } \\
\text { Elevation } \\
\text { (m-NAVD88) }\end{array}$ & $\begin{array}{l}\text { Elevation } \\
\text { Quality }^{2}\end{array}$ \\
\hline C4368 & 7.5 & AT-B-4-S & 4.440 & $\mathrm{BC} 5$ & $145,591.644$ & $565,845.890$ & SURV & GPS ID1552 FHI-TimJohnson-March04 & 119.662 & \\
\hline C4371 & 6.8 & AT-B-7-S & 4.620 & BC5 & $145,678.989$ & $566,142.321$ & SURV & GPS ID1555 FHI-TimJohnson-March04 & 119.493 & \\
\hline C4370 & 13.3 & AT-B-7-M & 4.620 & BC5 & $145,680.047$ & $566,143.324$ & SURV & GPS ID1554 FHI-TimJohnson-March04 & 119.343 & \\
\hline C4369 & 18.1 & AT-B-7-D & 4.620 & BC5 & $145,679.155$ & $566,143.550$ & SURV & GPS ID1553 FHI-TimJohnson-March04 & 119.549 & \\
\hline C4374 & 9.6 & AT-B-5-S & 4.770 & BC5 & $145,745.679$ & $566,435.772$ & SURV & GPS ID1562 FHI-TimJohnson-March04 & 119.330 & EST \\
\hline C4373 & 16.2 & AT-B-5-M & 4.770 & BC5 & $145,746.469$ & $566,436.650$ & SURV & GPS ID1556 FHI-TimJohnson-March04 & 119.340 & \\
\hline C4372 & 24.0 & AT-B-5-D & 4.770 & BC5 & $145,746.810$ & $566,438.064$ & SURV & GPS ID1557 FHI-TimJohnson-March04 & 119.320 & \\
\hline B8143 & 10.5 & $11-\mathrm{D}$ & 5.070 & BC5 & $145,903.913$ & $566,862.628$ & SURV & ERC-GPS ID\#99 (Nov/Dec 1997) & 119.300 & EST \\
\hline B8146 & 10.0 & 12-D & 5.330 & BC5 & $146,055.169$ & $567,231.722$ & SURV & ERC-GPS ID\#100 (Nov/Dec 1997) & 119.300 & EST \\
\hline B8151 & 8.3 & $13-S$ & 5.610 & $\mathrm{BC} 5$ & $146,172.598$ & $567,507.297$ & SURV & PNNL-GPS sub-meter fld recon (9/24/02) & 119.200 & EST \\
\hline B8149 & 22.9 & 13-D & 5.610 & BC5 & $146,172.598$ & $567,507.297$ & SURV & PNNL-GPS sub-meter fld recon (9/24/02) & 119.200 & EST \\
\hline B8154 & 7.5 & $14-S$ & 5.880 & KR4 & $146,268.095$ & $567,645.588$ & SURV & PNNL-GPS sub-meter fld recon (9/24/02) & 119.000 & EST \\
\hline B8153 & 14.5 & $14-\mathrm{M}$ & 5.880 & KR4 & $146,268.095$ & $567,645.588$ & SURV & PNNL-GPS sub-meter fld recon (9/24/02) & 119.000 & EST \\
\hline B8152 & 21.5 & 14-D & 5.880 & KR4 & $146,268.095$ & $567,645.588$ & SURV & PNNL-GPS sub-meter fld recon (9/24/02) & 119.000 & EST \\
\hline C6236 & 9.7 & C6236 & 6.040 & KR4 & $146,606.750$ & $568,031.670$ & SURV & AST Survey Data 1995-2008-MH-07509.xls & 118.480 & \\
\hline C6237 & 15.0 & C6237 & 6.040 & KR4 & $146,603.730$ & $568,031.500$ & SURV & AST Survey Data 1995-2008-MH-07509.xls & 118.700 & \\
\hline C6238 & 21.6 & C6238 & 6.040 & KR4 & $146,604.180$ & $568,033.650$ & SURV & AST Survey Data 1995-2008-MH-07509.xls & 118.800 & \\
\hline B8156 & 13.7 & $15-\mathrm{M}$ & 6.050 & KR4 & $146,602.100$ & $568,051.900$ & SURV & PNNL-field recon (10/01/03); sub-meter & 119.000 & EST \\
\hline C4341 & 9.2 & AT-K-1-S & 6.270 & KR4 & $146,805.775$ & $568,324.617$ & SURV & GPS ID1569 FHI-TimJohnson-March04 & 119.320 & \\
\hline C4340 & 15.0 & AT-K-1-M & 6.270 & KR4 & $146,806.936$ & $568,325.504$ & SURV & GPS ID1571 FHI-TimJohnson-March04 & 119.257 & \\
\hline C4339 & 21.7 & AT-K-1-D & 6.270 & KR4 & $146,807.110$ & $568,327.310$ & SURV & GPS ID1570 FHI-TimJohnson-March04 & 119.178 & \\
\hline B8162 & 11.0 & $17-\mathrm{M}$ & 6.370 & KR4 & $146,895.929$ & $568,480.003$ & SURV & PNNL-GPS sub-meter (11/20/02) & 119.000 & EST \\
\hline B8161 & 19.5 & $17-\mathrm{D}$ & 6.370 & KR4 & $146,895.929$ & $568,480.003$ & SURV & PNNL-GPS sub-meter (11/20/02) & 119.000 & EST \\
\hline C6239 & 10.2 & C6239 & 6.466 & KR4 & $146,979.250$ & $568,591.066$ & SURV & AST Survey Data 1995-2008-MH-07509.xls & 120.090 & \\
\hline C6240 & 14.9 & C6240 & 6.466 & KR4 & $146,980.255$ & $568,591.043$ & SURV & AST Survey Data 1995-2008-MH-07509.xls & 119.880 & \\
\hline C6241 & 21.8 & C6241 & 6.466 & KR4 & $146,979.656$ & $568,589.901$ & SURV & AST Survey Data 1995-2008-MH-07509.xls & 119.940 & \\
\hline C6244 & 27.5 & C6244 & 6.552 & KR4 & $147,065.122$ & $568,693.696$ & SURV & AST Survey Data 1995-2008-MH-07509.xls & 119.460 & \\
\hline C6242 & 12.7 & C6242 & 6.552 & KR4 & $147,063.409$ & $568,693.016$ & SURV & AST Survey Data 1995-2008-MH-07509.xls & 119.490 & \\
\hline C6243 & 20.7 & C6243 & 6.552 & KR4 & $147,063.896$ & $568,693.967$ & SURV & AST Survey Data 1995-2008-MH-07509.xls & 119.470 & \\
\hline B8204 & 8.5 & $18-\mathrm{S}$ & 6.560 & KR4 & $147,064.320$ & $568,712.600$ & SURV & PNNL-field recon (10/01/03); sub-meter & 119.000 & EST \\
\hline C4329 & 15.0 & AT-K-2-S & 6.640 & KR4 & $147,153.321$ & $568,804.249$ & SURV & GPS ID1576 FHI-TimJohnson-March04 & 119.427 & \\
\hline C4327 & 18.2 & AT-K-2-M & 6.640 & KR4 & $147,158.032$ & $568,803.992$ & SURV & GPS ID1575 FHI-TimJohnson-March04 & 119.181 & \\
\hline C4328 & 22.3 & AT-K-2-D & 6.640 & KR4 & $147,157.944$ & $568,801.243$ & SURV & GPS ID1577 FHI-TimJohnson-March04 & 118.857 & \\
\hline C6245 & 11.2 & C6245 & 6.750 & KR4 & $147,279.520$ & $568,922.440$ & SURV & AST Survey Data 1995-2008-MH-07509.xls & 117.970 & \\
\hline C6246 & 16.6 & C6246 & 6.750 & KR4 & $147,277.730$ & $568,920.870$ & SURV & AST Survey Data 1995-2008-MH-07509.xls & 117.940 & \\
\hline
\end{tabular}


Table 3.1. (contd)

\begin{tabular}{|c|c|c|c|c|c|c|c|c|c|c|}
\hline $\begin{array}{c}\text { Well } \\
\text { Identifier } \\
\text { (HWIS) }\end{array}$ & $\begin{array}{c}\text { Port } \\
\text { Depth } \\
\text { [to top of } \\
\text { opening] } \\
\text { (ft-bgs) }\end{array}$ & $\begin{array}{c}\text { Aquifer Tube } \\
\text { Name } \\
\text { (use in 2009) }\end{array}$ & $\begin{array}{c}\text { HRM } \\
\text { for Site } \\
\text { ("2k") }\end{array}$ & Segment $^{1}$ & $\begin{array}{c}\text { Northing } \\
\text { (m-NAD83) }\end{array}$ & $\begin{array}{c}\text { Easting } \\
\text { (m-NAD83) }\end{array}$ & $\begin{array}{c}\text { Coordinate } \\
\text { Quality }^{2}\end{array}$ & Coordinate Reference & $\begin{array}{c}\text { Ground } \\
\text { Elevation } \\
\text { (m-NAVD88) }\end{array}$ & $\begin{array}{l}\text { Elevation } \\
\text { Quality }^{2}\end{array}$ \\
\hline C6247 & 23.1 & C6247 & 6.750 & KR4 & $147,276.150$ & $568,921.680$ & SURV & AST Survey Data 1995-2008-MH-07509.xls & 117.950 & \\
\hline B8204 & 8.5 & $19-S$ & 6.860 & KR4 & & & & & & \\
\hline B8206 & 10.0 & $19-\mathrm{M}$ & 6.860 & KR4 & $147,389.952$ & $569,036.700$ & SURV & PNNL-GPS sub-meter (11/19/02) & 119.000 & EST \\
\hline B8205 & 22.0 & 19-D & 6.860 & KR4 & $147,389.952$ & $569,036.700$ & SURV & PNNL-GPS sub-meter $(11 / 19 / 02)$ & 119.000 & EST \\
\hline C4344 & 13.4 & AT-K-3-S & 7.000 & KR4 & $147,579.927$ & $569,177.923$ & SURV & GPS ID1573 FHI-TimJohnson-March04 & 118.333 & \\
\hline C4343 & 17.8 & AT-K-3-M & 7.000 & KR4 & $147,581.403$ & $569,180.026$ & SURV & GPS ID1574 FHI-TimJohnson-March04 & 118.266 & \\
\hline C4342 & 23.0 & AT-K-3-D & 7.000 & KR4 & $147,581.051$ & $569,178.215$ & SURV & GPS ID1572 FHI-TimJohnson-March04 & 118.109 & \\
\hline C6248 & 10.1 & C6248 & 7.120 & KR4 & $147,687.040$ & $569,313.260$ & SURV & AST Survey Data 1995-2008-MH-07509.xls & 118.510 & \\
\hline C6249 & 15.0 & C6249 & 7.120 & KR4 & $147,688.300$ & $569,309.540$ & SURV & AST Survey Data 1995-2008-MH-07509.xls & 118.250 & \\
\hline C6250 & 23.2 & C6250 & 7.120 & KR4 & $147,686.210$ & $569,314.980$ & SURV & AST Survey Data 1995-2008-MH-07509.xls & 118.610 & \\
\hline C6251 & 10.1 & C6251 & 7.180 & KR4 & $147,888.040$ & $569,467.170$ & SURV & AST Survey Data 1995-2008-MH-07509.xls & 117.640 & \\
\hline C6252 & 18.3 & C6252 & 7.180 & KR4 & $147,898.500$ & $569,475.540$ & SURV & AST Survey Data 1995-2008-MH-07509.xls & 117.600 & \\
\hline C6253 & 24.0 & C6253 & 7.180 & KR4 & $147,897.150$ & $569,464.260$ & SURV & AST Survey Data 1995-2008-MH-07509.xls & 117.240 & \\
\hline B8213 & 11.0 & $21-\mathrm{S}$ & 7.420 & KR4 & $148,020.334$ & $569,628.862$ & SURV & PNNL-GPS sub-meter fld recon (9/24/02) & 118.500 & EST \\
\hline B8212 & 15.0 & $21-\mathrm{M}$ & 7.420 & KR4 & $148,020.334$ & $569,628.862$ & SURV & PNNL-GPS sub-meter fld recon (9/24/02) & 118.500 & EST \\
\hline C6254 & 8.0 & C6254 & 7.540 & KR4 & $148,159.940$ & $569,713.150$ & SURV & AST Survey Data 1995-2008-MH-07509.xls & 117.980 & \\
\hline $\mathrm{C} 6255$ & 10.8 & $\mathrm{C} 6255$ & 7.540 & KR4 & $148,158.550$ & $569,709.360$ & SURV & AST Survey Data 1995-2008-MH-07509.xls & 117.890 & \\
\hline C6256 & 16.4 & C6256 & 7.540 & KR4 & $148,158.600$ & $569,711.500$ & SURV & AST Survey Data 1995-2008-MH-07509.xls & 117.930 & \\
\hline C6257 & 9.8 & C6257 & 7.660 & KR4 & $148,265.880$ & $569,853.390$ & SURV & AST Survey Data 1995-2008-MH-07509.xls & 119.030 & \\
\hline C6258 & 15.0 & C6258 & 7.660 & KR4 & $148,265.220$ & $569,851.350$ & SURV & AST Survey Data 1995-2008-MH-07509.xls & 118.980 & \\
\hline C6259 & 19.5 & C6259 & 7.660 & KR4 & $148,271.310$ & $569,850.480$ & SURV & AST Survey Data 1995-2008-MH-07509.xls & 118.770 & \\
\hline B8215 & 7.5 & $22-\mathrm{M}$ & 7.730 & KR4 & $148,340.349$ & $569,975.776$ & SURV & PNNL-GPS sub-meter fld recon (9/24/02) & 118.500 & EST \\
\hline B8214 & 12.3 & $22-\mathrm{D}$ & 7.730 & KR4 & $148,340.349$ & $569,975.776$ & SURV & PNNL-GPS sub-meter fld recon (9/24/02) & 118.500 & EST \\
\hline C4338 & 11.0 & AT-K-4-S & 7.880 & KR4 & $148,475.999$ & $570,122.433$ & SURV & GPS ID1567 FHI-TimJohnson-March04 & 118.973 & \\
\hline C4337 & 13.2 & AT-K-4-M & 7.880 & KR4 & $148,476.953$ & $570,123.114$ & SURV & GPS ID1565 FHI-TimJohnson-March04 & 118.977 & \\
\hline C4336 & 15.3 & AT-K-4-D & 7.880 & KR4 & $148,477.489$ & $570,123.757$ & SURV & GPS ID1564 FHI-TimJohnson-March04 & 118.996 & \\
\hline B8218 & 7.0 & 23-M & 7.950 & KR4 & $148,592.700$ & $570,225.500$ & SURV & PNNL-GPS submeter (5-2-05) & 118.800 & EST \\
\hline $\mathrm{B} 8217$ & 12.0 & 23-D & 7.950 & KR4 & $148,593.300$ & $570,228.000$ & SURV & PNNL-GPS submeter (5-2-05) & 118.800 & EST \\
\hline C6260 & 8.3 & C6260 & 7.990 & KR4 & $148,608.840$ & $570,214.850$ & SURV & AST Survey Data 1995-2008-MH-07509.xls & 118.190 & \\
\hline C6261 & 13.6 & C6261 & 7.990 & KR4 & $148,618.500$ & $570,209.670$ & SURV & AST Survey Data 1995-2008-MH-07509.xls & 118.020 & \\
\hline C4335 & 10.5 & AT-K-5-S & 8.090 & KR4 & $148,738.194$ & $570,357.990$ & SURV & GPS ID1562 FHI-TimJohnson-March04 & 118.837 & \\
\hline C4331 & 15.7 & AT-K-5-M & 8.090 & KR4 & $148,737.414$ & $570,356.510$ & SURV & GPS ID1563 FHI-TimJohnson-March04 & 118.728 & \\
\hline C4330 & 21.1 & AT-K-5-D & 8.090 & KR4 & $148,738.273$ & $570,358.927$ & SURV & GPS ID1561 FHI-TimJohnson-March04 & 118.837 & \\
\hline B8526 & 11.5 & DK-04-2 & 8.140 & KR4 & $148,872.300$ & $570,456.600$ & SURV & PNNL-GPS sub-meter (11/19/02) & 119.000 & EST \\
\hline B8527 & 15.0 & DK-04-3 & 8.140 & KR4 & $148,872.300$ & $570,456.600$ & SURV & PNNL-GPS sub-meter (11/19/02) & 119.000 & EST \\
\hline
\end{tabular}


Table 3.1. (contd)

\begin{tabular}{|c|c|c|c|c|c|c|c|c|c|c|c|}
\hline \multirow{36}{*}{$\stackrel{\omega}{v}$} & $\begin{array}{c}\text { Well } \\
\text { Identifier } \\
\text { (HWIS) }\end{array}$ & $\begin{array}{c}\text { Port } \\
\text { Depth } \\
\text { [to top of } \\
\text { opening] } \\
\text { (ft-bgs) }\end{array}$ & $\begin{array}{c}\text { Aquifer Tube } \\
\text { Name } \\
\text { (use in 2009) }\end{array}$ & $\begin{array}{l}\text { HRM } \\
\text { for Site } \\
\text { ("2k") }\end{array}$ & Segment $^{1}$ & $\begin{array}{l}\text { Northing } \\
\text { (m-NAD83) }\end{array}$ & $\begin{array}{c}\text { Easting } \\
\text { (m-NAD83) }\end{array}$ & $\begin{array}{c}\text { Coordinate } \\
\text { Quality }^{2}\end{array}$ & Coordinate Reference & $\begin{array}{c}\text { Ground } \\
\text { Elevation } \\
\text { (m-NAVD88) }\end{array}$ & $\begin{array}{l}\text { Elevation } \\
\text { Quality }^{2}\end{array}$ \\
\hline & B8223 & 7.5 & 25-D & 8.260 & KR4 & $148,960.980$ & $570,534.770$ & SURV & PNNL-GPS sub-meter (11/19/02) & 119.000 & EST \\
\hline & C4333 & 11.4 & AT-K-6-S & 8.310 & KR4 & $149,033.719$ & $570,641.237$ & SURV & GPS ID1559 FHI-TimJohnson-March04 & 120.073 & \\
\hline & C4334 & 15.3 & AT-K-6-M & 8.310 & KR4 & $149,032.947$ & $570,639.550$ & SURV & GPS ID1560 FHI-TimJohnson-March04 & 120.032 & \\
\hline & C4332 & 21.6 & AT-K-6-D & 8.310 & KR4 & $149,034.926$ & $570,642.437$ & SURV & GPS ID1558 FHI-TimJohnson-March04 & 119.962 & \\
\hline & B8228 & 6.0 & $26-S$ & 8.390 & KR4 & $149,115.832$ & $570,718.626$ & SURV & PNNL-GPS sub-meter $(11 / 20 / 02)$ & 120.000 & EST \\
\hline & B8227 & 14.0 & $26-\mathrm{M}$ & 8.390 & KR4 & $149,115.832$ & $570,718.626$ & SURV & PNNL-GPS sub-meter (11/20/02) & 120.000 & EST \\
\hline & B8226 & 23.0 & 26-D & 8.390 & KR4 & $149,115.832$ & $570,718.626$ & SURV & PNNL-GPS sub-meter (11/20/02) & 120.000 & EST \\
\hline & C6263 & 12.8 & C6263 & 8.539 & NR2 & $149,364.420$ & $570,887.320$ & SURV & AST Survey Data 1995-2008-MH-07509.xls & 118.890 & \\
\hline & C6264 & 20.2 & C6264 & 8.540 & NR2 & $149,363.590$ & $570,887.190$ & SURV & AST Survey Data 1995-2008-MH-07509.xls & 119.020 & \\
\hline & C6265 & 27.3 & C6265 & 8.541 & NR2 & $149,365.430$ & $570,886.330$ & SURV & AST Survey Data 1995-2008-MH-07509.xls & 118.740 & \\
\hline & C6317 & 7.9 & $\mathrm{C} 6317$ & 8.660 & NR2 & $149,554.570$ & $571,025.320$ & SURV & AST Survey Data 1995-2008-MH-07509.xls & 117.640 & \\
\hline & C6318 & 13.5 & $\mathrm{C} 6318$ & 8.661 & NR2 & $149,554.800$ & $571,026.840$ & SURV & AST Survey Data 1995-2008-MH-07509.xls & 117.770 & \\
\hline & C6319 & 22.2 & C6319 & 8.662 & NR2 & $149,554.400$ & $571,023.870$ & SURV & AST Survey Data 1995-2008-MH-07509.xls & 117.600 & \\
\hline & C6320 & 8.5 & C6320 & 8.746 & NR2 & $149,677.990$ & $571,128.660$ & SURV & AST Survey Data 1995-2008-MH-07509.xls & 118.320 & \\
\hline & C6321 & 12.6 & C6321 & 8.747 & NR2 & $149,677.460$ & $571,128.660$ & SURV & AST Survey Data 1995-2008-MH-07509.xls & 118.380 & \\
\hline & C6352 & 14.1 & C6352 & 8.748 & NR2 & $149,678.602$ & $571,129.473$ & SURV & AST Survey Data 1995-2008-MH-07509.xls & 118.380 & \\
\hline & C6322 & 18.8 & C6322 & 8.748 & NR2 & $149,679.680$ & $571,127.030$ & SURV & AST Survey Data 1995-2008-MH-07509.xls & 117.670 & \\
\hline & C6131 & 4.5 & C6131 & 8.791 & NR2 & $149,733.710$ & $571,153.810$ & SURV & AST Survey Data 1995-2008-MH-07509.xls & 117.600 & \\
\hline & C6132 & 5.5 & C6132 & 8.795 & NR2 & $149,734.960$ & $571,165.270$ & SURV & AST Survey Data 1995-2008-MH-07509.xls & 117.670 & \\
\hline & C6133 & 2.7 & C6133 & 8.813 & NR2 & $149,776.130$ & $571,168.610$ & SURV & AST Survey Data 1995-2008-MH-07509.xls & 117.600 & \\
\hline & C5514 & 5.4 & N116mArray-0A & 8.815 & NR2 & $149,776.131$ & $571,168.610$ & & PNNL-GPS xyz from Brad Fritz, 11/16/06 & 117.649 & \\
\hline & C6134 & 2.5 & C6134 & 8.825 & NR2 & $149,794.070$ & $571,181.410$ & SURV & AST Survey Data 1995-2008-MH-07509.xls & 117.600 & \\
\hline & C6135 & 4.9 & C6135 & 8.825 & NR2 & $149,794.070$ & $571,181.410$ & SURV & AST Survey Data 1995-2008-MH-07509.xls & 117.600 & \\
\hline & C6136 & 4.9 & C6136 & 8.393 & NR2 & $149,815.360$ & $571,195.690$ & SURV & AST Survey Data 1995-2008-MH-07509.xls & 117.600 & \\
\hline & C5255 & 3.9 & N116mArray-1A & 8.865 & NR2 & $149,860.200$ & $571,224.900$ & SURV & PNNL-BradFritz-(file 10/13/06) & 117.200 & \\
\hline & C5256 & 2.0 & N116mArray-2A & 8.882 & NR2 & $149,888.375$ & $571,259.688$ & & PNNL-GPS xyz from Brad Fritz, 9/1/06 & 116.630 & \\
\hline & C5269 & 8.9 & APT1 & 8.891 & NR2 & $149,897.484$ & $571,276.500$ & SURV & PNNL-GPS xyz from Brad Fritz, 9/1/06 & 118.860 & \\
\hline & C5270 & 5.2 & APT2 & 8.892 & NR2 & $149,899.578$ & $571,275.313$ & SURV & PNNL-GPS xyz from Brad Fritz, 9/1/06 & 118.002 & \\
\hline & C5271 & 2.8 & APT3 & 8.893 & NR2 & $149,905.047$ & $571,270.563$ & SURV & PNNL-GPS xyz from Brad Fritz, 9/1/06 & 117.008 & \\
\hline & C5257 & 2.0 & N116mArray-3A & 8.895 & NR2 & $149,911.859$ & $571,273.375$ & & PNNL-GPS xyz from Brad Fritz, 9/1/06 & 116.600 & \\
\hline & C5258 & 3.3 & N116mArray-4A & 8.907 & NR2 & $149,925.234$ & $571,292.500$ & & PNNL-GPS xyz from Brad Fritz, 9/1/06 & 117.000 & \\
\hline & C4585 & 0.8 & NS-2A-23cm & 8.910 & NR2 & $149,925.438$ & $571,292.325$ & SURV & PNNL-GPS xyz from Brad Fritz, 9/1/06 & 116.988 & \\
\hline & C4586 & 2.9 & NS-2A-87cm & 8.910 & NR2 & $149,925.438$ & $571,292.325$ & SURV & PNNL-GPS xyz from Brad Fritz, 9/1/06 & 116.970 & \\
\hline & C4587 & 5.5 & NS-2A-168cm & 8.910 & NR2 & $149,925.438$ & $571,292.325$ & SURV & PNNL-GPS xyz from Brad Fritz, 9/1/06 & 116.925 & \\
\hline & C5245 & 3.3 & NVP1-1 & 8.918 & NR2 & $149,935.703$ & $571,317.500$ & & PNNL-GPS xyz from Brad Fritz, 9/1/06 & 119.000 & \\
\hline
\end{tabular}


Table 3.1. (contd)

\begin{tabular}{|c|c|c|c|c|c|c|c|c|c|c|}
\hline $\begin{array}{c}\text { Well } \\
\text { Identifier } \\
\text { (HWIS) }\end{array}$ & $\begin{array}{c}\text { Port } \\
\text { Depth } \\
\text { [to top of } \\
\text { opening] } \\
\text { (ft-bgs) }\end{array}$ & $\begin{array}{c}\text { Aquifer Tube } \\
\text { Name } \\
\text { (use in 2009) }\end{array}$ & $\begin{array}{c}\text { HRM } \\
\text { for Site } \\
\text { ("2k") }\end{array}$ & Segment $^{1}$ & $\begin{array}{c}\text { Northing } \\
\text { (m-NAD83) }\end{array}$ & $\begin{array}{c}\text { Easting } \\
\text { (m-NAD83) }\end{array}$ & $\begin{array}{c}\text { Coordinate } \\
\text { Quality }^{2}\end{array}$ & Coordinate Reference & $\begin{array}{c}\text { Ground } \\
\text { Elevation } \\
\text { (m-NAVD88) }\end{array}$ & $\begin{array}{l}\text { Elevation } \\
\text { Quality }^{2}\end{array}$ \\
\hline C5246 & 4.1 & NVP1-2 & 8.918 & NR2 & $149,935.703$ & $571,317.500$ & & PNNL-GPS xyz from Brad Fritz, 9/1/06 & 119.000 & \\
\hline C5247 & 5.6 & NVP1-3 & 8.918 & NR2 & $149,935.703$ & $571,317.500$ & & PNNL-GPS xyz from Brad Fritz, 9/1/06 & 119.000 & \\
\hline C4588 & 0.3 & NS-3A-10cm & 8.919 & NR2 & $149,941.215$ & $571,310.501$ & SURV & PNNL-GPS xyz from Brad Fritz, 9/1/06 & 116.978 & \\
\hline C4590 & 2.9 & NS-3A-87cm & 8.919 & NR2 & $149,941.215$ & $571,310.501$ & SURV & PNNL-GPS xyz from Brad Fritz, 9/1/06 & 116.958 & \\
\hline C4589 & 5.8 & NS-3A- $176 \mathrm{~cm}$ & 8.919 & NR2 & $149,941.215$ & $571,310.501$ & SURV & PNNL-GPS xyz from Brad Fritz, 9/1/06 & 117.020 & \\
\hline C4892 & 1.3 & NS-3B-40cm & 8.921 & NR2 & $149,947.000$ & $571,316.000$ & EST & PNNL-GPS xyz from Brad Fritz, 9/1/06 & 115.880 & \\
\hline C4893 & 1.7 & NS-3B-52cm & 8.921 & NR2 & $149,947.000$ & $571,316.000$ & EST & PNNL-GPS xyz from Brad Fritz, 9/1/06 & 115.880 & \\
\hline C5248 & 5.7 & NVP1-4 & 8.922 & NR2 & $149,939.094$ & $571,317.438$ & & PNNL-GPS xyz from Brad Fritz, 9/1/06 & 118.500 & \\
\hline C5250 & 2.3 & NVP2-116.3 & 8.924 & NR2 & $149,944.563$ & $571,313.313$ & & PNNL-GPS xyz from Brad Fritz, 9/1/06 & 117.000 & \\
\hline C5251 & 3.3 & NVP2-116.0 & 8.924 & NR2 & $149,944.563$ & $571,313.313$ & & PNNL-GPS xyz from Brad Fritz, 9/1/06 & 117.000 & \\
\hline C5252 & 4.3 & NVP2-115.7 & 8.924 & NR2 & $149,944.563$ & $571,313.313$ & & PNNL-GPS xyz from Brad Fritz, 9/1/06 & 117.000 & \\
\hline C5253 & 5.3 & NVP2-115.4 & 8.924 & NR2 & $149,944.563$ & $571,313.313$ & & PNNL-GPS xyz from Brad Fritz, 9/1/06 & 117.000 & \\
\hline C5254 & 6.2 & NVP2-115.1 & 8.924 & NR2 & $149,944.563$ & $571,313.313$ & & PNNL-GPS xyz from Brad Fritz, 9/1/06 & 117.000 & \\
\hline C5249 & 7.2 & NVP1-5 & 8.925 & NR2 & $149,945.875$ & $571,317.375$ & & PNNL-GPS xyz from Brad Fritz, 9/1/06 & 118.500 & \\
\hline C5259 & 2.3 & N116mArray-6A & 8.931 & NR2 & $149,955.734$ & $571,325.438$ & & PNNL-GPS xyz from Brad Fritz, 9/1/06 & 116.700 & \\
\hline C4640 & 0.6 & $\mathrm{NS}-4 \mathrm{~A}-17 \mathrm{~cm}$ & 8.932 & NR2 & $149,954.770$ & $571,328.703$ & SURV & GPS xyz from Brad Fritz, 9/1/06 & 117.289 & \\
\hline C4641 & 4.5 & NS-4A-138cm & 8.932 & NR2 & $149,954.770$ & $571,328.703$ & SURV & GPS xyz from Brad Fritz, 9/1/06 & 117.229 & \\
\hline C4894 & 1.0 & NS-4B-31cm & 8.933 & NR2 & $149,959.000$ & $571,328.000$ & EST & GPS xyz from Brad Fritz, 9/1/06 & & \\
\hline (none) & 5.3 & APT5S & 8.935 & NR2 & $149,957.200$ & $571,334.100$ & SURV & PNNL-BradFritz-(file 10/13/06); install est. & & \\
\hline (none) & 10.0 & APT5 & 8.935 & NR2 & $149,957.200$ & $571,334.100$ & SURV & PNNL-BradFritz-(file 10/13/06); install est. & & \\
\hline C5260 & 3.0 & N116mArray-7A & 8.950 & NR2 & $149,981.844$ & $571,346.688$ & & PNNL-GPS xyz from Brad Fritz, 9/1/06 & 116.900 & \\
\hline C5261 & 3.3 & N116mArray-8A & 8.983 & NR2 & $150,029.750$ & $571,381.188$ & & PNNL-GPS xyz from Brad Fritz, 9/1/06 & 117.000 & \\
\hline C5262 & & N116mArray-8.5A & 9.012 & NR2 & $150,074.547$ & $571,404.563$ & & PNNL-GPS xyz from Brad Fritz, 9/1/06 & 117.070 & \\
\hline C5263 & 3.3 & N116mArray-9A & 9.044 & NR2 & $150,124.984$ & $571,437.813$ & & PNNL-GPS xyz from Brad Fritz, 9/1/06 & 117.000 & \\
\hline C5264 & & N116mArray-10A & 9.065 & NR2 & $150,158.656$ & $571,452.000$ & & PNNL-GPS xyz from Brad Fritz, 9/1/06 & & \\
\hline C5265 & & N116mArray-11A & 9.090 & NR2 & $150,184.453$ & $571,482.063$ & & PNNL-GPS xyz from Brad Fritz, 9/1/06 & & \\
\hline C5266 & & N116mArray-12A & 9.118 & NR2 & $150,219.156$ & $571,512.000$ & & PNNL-GPS xyz from Brad Fritz, 9/1/06 & 117.060 & \\
\hline C5267 & & N116mArray-13A & 9.149 & NR2 & $150,264.016$ & $571,540.688$ & & PNNL-GPS xyz from Brad Fritz, 9/1/06 & 117.580 & \\
\hline $\mathrm{C} 6323$ & 7.7 & C6323 & 9.161 & NR2 & $150,269.680$ & $571,555.040$ & SURV & AST Survey Data 1995-2008-MH-07509.xls & 117.730 & \\
\hline C6324 & 14.2 & C6324 & 9.161 & NR2 & $150,268.720$ & $571,554.350$ & SURV & AST Survey Data 1995-2008-MH-07509.xls & 117.700 & \\
\hline C6325 & 23.4 & $\mathrm{C} 6325$ & 9.161 & NR2 & $150,267.910$ & $571,553.390$ & SURV & AST Survey Data 1995-2008-MH-07509.xls & 117.540 & \\
\hline C5268 & & N116mArray-14A & 9.183 & NR2 & $150,311.047$ & $571,573.375$ & & PNNL-GPS xyz from Brad Fritz, 9/1/06 & 117.600 & \\
\hline C5512 & 5.5 & N116mArray-15A & 9.217 & NR2 & $150,390.786$ & $571,631.861$ & & PNNL-GPS xyz from Brad Fritz, 11/16/06 & 117.650 & \\
\hline C5513 & 5.4 & N116mArray-16A & 9.251 & NR2 & $150,491.336$ & $571,674.274$ & & PNNL-GPS xyz from Brad Fritz, 11/16/06 & 117.686 & \\
\hline C6326 & 9.9 & C6326 & 9.357 & NR2 & $150,540.300$ & $571,738.800$ & SURV & AST Survey Data 1995-2008-MH-07509.xls & 118.160 & \\
\hline
\end{tabular}


Table 3.1. (contd)

\begin{tabular}{|c|c|c|c|c|c|c|c|c|c|c|}
\hline $\begin{array}{c}\text { Well } \\
\text { Identifier } \\
\text { (HWIS) }\end{array}$ & $\begin{array}{c}\text { Port } \\
\text { Depth } \\
\text { [to top of } \\
\text { opening] } \\
\text { (ft-bgs) }\end{array}$ & $\begin{array}{c}\text { Aquifer Tube } \\
\text { Name } \\
\text { (use in 2009) }\end{array}$ & $\begin{array}{c}\text { HRM } \\
\text { for Site } \\
\text { ("2k") }\end{array}$ & Segment $^{1}$ & $\begin{array}{c}\text { Northing } \\
\text { (m-NAD83) }\end{array}$ & $\begin{array}{c}\text { Easting } \\
\text { (m-NAD83) }\end{array}$ & $\begin{array}{c}\text { Coordinate } \\
\text { Quality }^{2}\end{array}$ & Coordinate Reference & $\begin{array}{c}\text { Ground } \\
\text { Elevation } \\
\text { (m-NAVD88) }\end{array}$ & $\begin{array}{l}\text { Elevation } \\
\text { Quality }^{2}\end{array}$ \\
\hline C6327 & 16.7 & C6327 & 9.357 & NR2 & $150,540.590$ & $571,740.710$ & SURV & AST Survey Data 1995-2008-MH-07509.xls & 118.330 & \\
\hline C6328 & 24.8 & C6328 & 9.357 & NR2 & $150,541.110$ & $571,741.350$ & SURV & AST Survey Data 1995-2008-MH-07509.xls & 118.270 & \\
\hline C6329 & 15.6 & C6329 & 9.491 & NR2 & $150,718.710$ & $571,871.470$ & SURV & AST Survey Data 1995-2008-MH-07509.xls & 117.580 & \\
\hline $\mathrm{C6330}$ & 22.1 & $\mathrm{C6330}$ & 9.491 & NR2 & $150,718.880$ & $571,872.000$ & SURV & AST Survey Data 1995-2008-MH-07509.xls & 117.550 & \\
\hline C6331 & 28.7 & C6331 & 9.491 & NR2 & $150,720.490$ & $571,872.770$ & SURV & AST Survey Data 1995-2008-MH-07509.xls & 117.490 & \\
\hline $\mathrm{C} 6332$ & 9.8 & $\mathrm{C} 6332$ & 9.670 & NR2 & $150,949.080$ & $572,045.850$ & SURV & AST Survey Data 1995-2008-MH-07509.xls & 117.410 & \\
\hline $\mathrm{C} 6333$ & 17.1 & $\mathrm{C6333}$ & 9.670 & NR2 & $150,948.240$ & $572,045.340$ & SURV & AST Survey Data 1995-2008-MH-07509.xls & 117.320 & \\
\hline C6334 & 24.7 & $\mathrm{C} 6334$ & 9.670 & NR2 & $150,949.920$ & $572,046.290$ & SURV & AST Survey Data 1995-2008-MH-07509.xls & 117.280 & \\
\hline B8515 & 15.0 & DD-50-1 & 9.800 & HR3D & $151,121.164$ & $572,172.189$ & SURV & ERC-GPS ID\#1034 (Sep 1996) & 119.454 & \\
\hline B8516 & 20.0 & DD-50-2 & 9.800 & HR3D & $151,121.569$ & $572,172.633$ & SURV & ERC-GPS ID\#1035 (Sep 1996) & 119.478 & \\
\hline B8517 & 24.7 & DD-50-3 & 9.800 & HR3D & $151,120.007$ & $572,172.166$ & SURV & ERC-GPS ID\#1033 (Sep 1996) & 119.472 & \\
\hline B8518 & 31.0 & DD-50-4 & 9.800 & HR3D & $151,121.180$ & $572,173.030$ & SURV & ERC-GPS ID\#1036 (Sep 1996) & 119.514 & \\
\hline B8511 & 12.0 & DD-49-1 & 9.830 & HR3D & $151,161.553$ & $572,213.696$ & SURV & ERC-GPS ID\#1030 (Sep 1996) & 119.627 & \\
\hline B8512 & 15.8 & DD-49-2 & 9.830 & HR3D & $151,162.781$ & $572,213.968$ & SURV & ERC-GPS ID\#1031 (Sep 1996) & 119.492 & \\
\hline B8513 & 25.0 & DD-49-3 & 9.830 & HR3D & $151,163.304$ & $572,210.802$ & SURV & ERC-GPS ID\#1028 (Sep 1996) & 118.557 & \\
\hline B8513a & 25.0 & DD-49-3a & 9.830 & HR3D & & & & & 118.557 & EST \\
\hline B8514 & 31.0 & DD-49-4 & 9.830 & HR3D & $151,163.981$ & $572,211.150$ & SURV & ERC-GPS ID\#1029 (Sep 1996) & 118.487 & \\
\hline C6266 & 9.6 & C6266 & 9.890 & HR3D & $151,241.630$ & $572,269.900$ & SURV & AST Survey Data 1995-2008-MH-07509.xls & 117.680 & \\
\hline C6267 & 12.7 & C6267 & 9.890 & HR3D & $151,242.230$ & $572,270.420$ & SURV & AST Survey Data 1995-2008-MH-07509.xls & 117.630 & \\
\hline C6268 & 21.7 & C6268 & 9.890 & HR3D & $151,246.190$ & $572,274.210$ & SURV & AST Survey Data 1995-2008-MH-07509.xls & 117.620 & \\
\hline C6269 & 8.0 & C6269 & 9.952 & HR3D & $151,323.200$ & $572,335.820$ & SURV & AST Survey Data 1995-2008-MH-07509.xls & 117.430 & \\
\hline C6270 & 12.8 & C6270 & 9.952 & HR3D & $151,324.520$ & $572,337.130$ & SURV & AST Survey Data 1995-2008-MH-07509.xls & 117.620 & \\
\hline C6271 & 18.3 & C6271 & 9.952 & HR3D & $151,317.570$ & $572,332.300$ & SURV & AST Survey Data 1995-2008-MH-07509.xls & 117.470 & \\
\hline B8509 & 12.0 & DD-44-3 & 10.010 & HR3D & $151,394.279$ & $572,412.676$ & SURV & ERC-GPS ID\#1022 (Sep 1996) & 118.199 & \\
\hline B8510 & 18.0 & DD-44-4 & 10.010 & HR3D & $151,396.947$ & $572,411.362$ & SURV & ERC-GPS ID\#1021 (Sep 1996) & 117.645 & \\
\hline B8507 & 10.0 & DD-43-2 & 10.050 & HR3D & $151,443.222$ & $572,450.906$ & SURV & ERC-GPS ID\#1018 (Sep 1996) & 117.536 & \\
\hline B8508 & 13.9 & DD-43-3 & 10.050 & HR3D & $151,443.236$ & $572,450.504$ & SURV & ERC-GPS ID\#1019 (Sep 1996) & 117.521 & \\
\hline B8504 & 10.2 & DD-42-2 & 10.090 & HR3D & $151,492.564$ & $572,485.766$ & SURV & ERC-GPS ID\#1013 (Sep 1996) & 117.919 & \\
\hline B8505 & 15.2 & DD-42-3 & 10.090 & HR3D & $151,492.480$ & $572,486.189$ & SURV & ERC-GPS ID\#1014 (Sep 1996) & 117.939 & \\
\hline B8506 & 18.2 & DD-42-4 & 10.090 & HR3D & $151,492.503$ & $572,485.319$ & SURV & ERC-GPS ID\#1015 (Sep 1996) & 117.973 & \\
\hline B8503 & 8.1 & DD-41-1 & 10.124 & HR3D & $151,530.892$ & $572,532.894$ & SURV & Unknown & 117.689 & \\
\hline B8483 & 13.6 & DD-41-2 & 10.124 & HR3D & $151,530.738$ & $572,532.688$ & SURV & ERC-GPS ID\#1009 (Sep 1996) & 117.684 & \\
\hline B8484 & 18.6 & DD-41-3 & 10.124 & HR3D & $151,530.033$ & $572,532.209$ & SURV & ERC-GPS ID\#1011 (Sep 1996) & 117.646 & \\
\hline C3383 & 3.0 & Redox-4-3.0 & 10.125 & HR3D & $151,540.006$ & $572,529.706$ & SURV & PNNL-E. Arntzen (1999-2000) & 116.600 & EST \\
\hline C3515 & 6.0 & Redox-4-6.0 & 10.125 & HR3D & $151,540.006$ & $572,529.706$ & SURV & PNNL-E. Arntzen (1999-2000) & 116.600 & EST \\
\hline
\end{tabular}


Table 3.1. (contd)

\begin{tabular}{|c|c|c|c|c|c|c|c|c|c|c|}
\hline $\begin{array}{c}\text { Well } \\
\text { Identifier } \\
\text { (HWIS) }\end{array}$ & $\begin{array}{c}\text { Port } \\
\text { Depth } \\
\text { [to top of } \\
\text { opening] } \\
\text { (ft-bgs) }\end{array}$ & $\begin{array}{c}\text { Aquifer Tube } \\
\text { Name } \\
\text { (use in 2009) }\end{array}$ & $\begin{array}{l}\text { HRM } \\
\text { for Site } \\
\text { ("2k") }\end{array}$ & Segment $^{1}$ & $\begin{array}{c}\text { Northing } \\
\text { (m-NAD83) }\end{array}$ & $\begin{array}{c}\text { Easting } \\
\text { (m-NAD83) }\end{array}$ & $\begin{array}{l}\text { Coordinate } \\
\text { Quality }^{2}\end{array}$ & Coordinate Reference & $\begin{array}{c}\text { Ground } \\
\text { Elevation } \\
\text { (m-NAVD88) }\end{array}$ & $\begin{array}{c}\text { Elevation } \\
\text { Quality }^{2}\end{array}$ \\
\hline C3384 & 3.3 & Redox-3-3.3 & 10.180 & HR3D & $151,603.027$ & $572,583.206$ & SURV & PNNL-E. Arntzen (1999-2000) & 116.900 & EST \\
\hline C3514 & 4.6 & Redox-3-4.6 & 10.180 & HR3D & $151,603.027$ & $572,583.206$ & SURV & PNNL-E. Arntzen (1999-2000) & 116.900 & EST \\
\hline B8479 & 5.5 & DD-39-1 & 10.210 & HR3D & $151,625.856$ & $572,606.547$ & SURV & ERC-GPS ID\#1005 (Sep 1996) & 117.913 & \\
\hline B8480 & 10.5 & DD-39-2 & 10.210 & HR3D & $151,626.189$ & $572,606.894$ & SURV & ERC-GPS ID\#1006 (Sep 1996) & 117.940 & \\
\hline B8481 & 15.0 & DD-39-3 & 10.210 & HR3D & $151,626.197$ & $572,606.548$ & SURV & ERC-GPS ID\#1007 (Sep 1996) & 117.883 & \\
\hline B8482 & 21.0 & DD-39-4 & 10.210 & HR3D & $151,625.527$ & $572,606.888$ & SURV & ERC-GPS ID\#1004 (Sep 1996) & 118.012 & \\
\hline C3385 & 3.0 & Redox-2-3.0 & 10.240 & HR3D & $151,687.803$ & $572,636.662$ & SURV & PNNL-E. Arntzen (1999-2000) & 117.000 & EST \\
\hline C3513 & 6.0 & Redox-2-6.0 & 10.240 & HR3D & $151,687.803$ & $572,636.662$ & SURV & PNNL-E. Arntzen (1999-2000) & 117.000 & EST \\
\hline C3382 & 3.3 & Redox-1-3.3 & 10.295 & HR3D & $151,730.810$ & $572,716.336$ & SURV & PNNL-E. Arntzen (1999-2000) & 117.000 & EST \\
\hline C3512 & 6.0 & Redox-1-6.0 & 10.295 & HR3D & $151,730.810$ & $572,716.336$ & SURV & PNNL-E. Arntzen (1999-2000) & 117.000 & EST \\
\hline C4307 & 7.0 & AT-D-1-S & 10.420 & HR3D & $151,850.983$ & $572,861.059$ & SURV & GPS ID1579 FHI-TimJohnson-March04 & 118.536 & \\
\hline C4306 & 10.8 & AT-D-1-M & 10.420 & HR3D & $151,852.065$ & $572,860.651$ & SURV & GPS ID1580 FHI-TimJohnson-March04 & 118.286 & \\
\hline C4305 & 13.3 & AT-D-1-D & 10.420 & HR3D & $151,850.404$ & $572,861.512$ & SURV & GPS ID1578 FHI-TimJohnson-March04 & 118.662 & \\
\hline C6272 & 9.7 & C6272 & 10.479 & HR3D & $151,926.860$ & $572,920.420$ & SURV & AST Survey Data 1995-2008-MH-07509.xls & 118.710 & \\
\hline B8255 & 8.0 & $35-S$ & 10.480 & HR3D & $151,929.120$ & $572,917.880$ & SURV & PNNL-field recon (10/01/03) & 118.600 & EST \\
\hline B8254 & 14.0 & $35-\mathrm{M}$ & 10.480 & HR3D & $151,929.120$ & $572,917.880$ & SURV & PNNL-field recon (10/01/03) & 118.600 & EST \\
\hline B8253 & 21.0 & $35-\mathrm{D}$ & 10.480 & HR3D & $151,929.120$ & $572,917.880$ & SURV & PNNL-field recon (10/01/03) & 118.600 & EST \\
\hline C4314 & 12.4 & AT-D-4-S & 10.580 & HR3D & $152,039.864$ & $572,993.254$ & SURV & GPS ID3009 FHI-TimJohnson-March04 & 118.878 & \\
\hline C4315 & 13.8 & AT-D-4-M & 10.580 & HR3D & $152,040.484$ & $572,993.148$ & SURV & GPS ID3010 FHI-TimJohnson-March04 & 118.718 & \\
\hline C4316 & 15.7 & AT-D-4-D & 10.580 & HR3D & $152,039.564$ & $572,992.239$ & SURV & GPS ID3008 FHI-TimJohnson-March04 & 118.718 & \\
\hline $\mathrm{C} 4310$ & 14.3 & AT-D-2-S & 10.660 & HR3D & $152,129.777$ & $573,108.251$ & SURV & GPS ID3007 FHI-TimJohnson-March04 & 118.738 & \\
\hline C4309 & 16.3 & AT-D-2-M & 10.660 & HR3D & $152,130.073$ & $573,105.575$ & SURV & GPS ID3005 FHI-TimJohnson-March04 & 118.358 & \\
\hline C4308 & 26.5 & AT-D-2-D & 10.660 & HR3D & $152,130.318$ & $573,105.776$ & SURV & GPS ID3006 FHI-TimJohnson-March04 & 118.358 & \\
\hline B8258 & 8.0 & $36-S$ & 10.665 & HR3D & $152,146.430$ & $573,120.650$ & SURV & PNNL-field recon (10/01/03) & 118.700 & EST \\
\hline B8257 & 14.0 & $36-\mathrm{M}$ & 10.665 & HR3D & $152,146.430$ & $573,120.650$ & SURV & PNNL-field recon (10/01/03) & 118.700 & EST \\
\hline B8256 & 21.0 & 36-D & 10.665 & HR3D & $152,146.430$ & $573,120.650$ & SURV & PNNL-field recon (10/01/03) & 118.700 & EST \\
\hline C4313 & 7.3 & AT-D-3-S & 10.750 & HR3D & $152,224.602$ & $573,233.717$ & SURV & GPS ID3003 FHI-TimJohnson-March04 & 118.654 & \\
\hline $\mathrm{C} 4312$ & 8.8 & AT-D-3-M & 10.750 & HR3D & $152,224.974$ & $573,234.706$ & SURV & GPS ID3004 FHI-TimJohnson-March04 & 118.652 & \\
\hline C4311 & 11.8 & AT-D-3-D & 10.750 & HR3D & $152,224.319$ & $573,233.231$ & SURV & GPS ID3002 FHI-TimJohnson-March04 & 118.681 & \\
\hline B8261 & 6.5 & $37-S$ & 10.825 & HR3D & $152,287.340$ & $573,335.960$ & SURV & PNNL-field recon (10/01/03) & 118.000 & EST \\
\hline B8260 & 13.5 & $37-M$ & 10.825 & HR3D & $152,287.340$ & $573,335.960$ & SURV & PNNL-field recon (10/01/03) & 118.000 & EST \\
\hline B8259 & 19.5 & $37-\mathrm{D}$ & 10.825 & HR3D & $152,287.340$ & $573,335.960$ & SURV & PNNL-field recon (10/01/03) & 118.000 & EST \\
\hline $\mathrm{C} 6275$ & 10.4 & C6275 & 10.832 & HR3D & $152,284.930$ & $573,337.490$ & SURV & AST Survey Data 1995-2008-MH-07509.xls & 117.930 & \\
\hline B8263 & 10.0 & 38-M & 10.920 & HR3D & $152,368.720$ & $573,471.850$ & SURV & PNNL-field recon (10/01/03) & 118.000 & EST \\
\hline B8262 & 16.5 & 38-D & 10.920 & HR3D & $152,368.720$ & $573,471.850$ & SURV & PNNL-field recon (10/01/03) & 118.000 & EST \\
\hline
\end{tabular}


Table 3.1. (contd)

\begin{tabular}{|c|c|c|c|c|c|c|c|c|c|c|}
\hline $\begin{array}{c}\text { Well } \\
\text { Identifier } \\
\text { (HWIS) }\end{array}$ & $\begin{array}{c}\text { Port } \\
\text { Depth } \\
\text { [to top of } \\
\text { opening] } \\
\text { (ft-bgs) }\end{array}$ & $\begin{array}{l}\text { Aquifer Tube } \\
\text { Name } \\
\text { (use in 2009) }\end{array}$ & $\begin{array}{l}\text { HRM } \\
\text { for Site } \\
\text { ("2k") }\end{array}$ & Segment $^{1}$ & $\begin{array}{c}\text { Northing } \\
\text { (m-NAD83) }\end{array}$ & $\begin{array}{c}\text { Easting } \\
\text { (m-NAD83) }\end{array}$ & $\begin{array}{l}\text { Coordinate } \\
\text { Quality }^{2}\end{array}$ & Coordinate Reference & $\begin{array}{c}\text { Ground } \\
\text { Elevation } \\
\text { (m-NAVD88) }\end{array}$ & $\begin{array}{c}\text { Elevation } \\
\text { Quality }^{2}\end{array}$ \\
\hline B8477 & 10.5 & DD-17-2 & 11.030 & HR3D & $152,482.772$ & $573,597.984$ & SURV & ERC-GPS ID\#1042 (Sep 1996) & 117.390 & \\
\hline B8478 & 15.0 & DD-17-3 & 11.030 & HR3D & $152,483.304$ & $573,597.162$ & SURV & ERC-GPS ID\#1041 (Sep 1996) & 117.313 & \\
\hline C6278 & 9.7 & C6278 & 11.041 & HR3D & $152,429.805$ & $573,532.396$ & SURV & Garmin V by Rich Mahood & & \\
\hline B8475 & 17.5 & DD-16-3 & 11.060 & HR3D & $152,516.128$ & $573,650.431$ & SURV & ERC-GPS ID\#1045 (Sep 1996) & 120.013 & \\
\hline B8476 & 25.5 & DD-16-4 & 11.060 & HR3D & $152,516.993$ & $573,651.165$ & SURV & ERC-GPS ID\#1046 (Sep 1996) & 119.965 & \\
\hline B8472 & 15.0 & DD-15-2 & 11.100 & HR3D & $152,547.828$ & $573,700.609$ & SURV & ERC-GPS ID\#1049 (Sep 1996) & 119.316 & \\
\hline B8473 & 21.0 & DD-15-3 & 11.100 & HR3D & $152,547.828$ & $573,700.609$ & SURV & ERC-GPS ID\#1048 (Sep 1996) & 119.365 & \\
\hline B8474 & 25.5 & DD-15-4 & 11.100 & HR3D & $152,547.828$ & $573,700.609$ & SURV & ERC-GPS ID\#1050 (Sep 1996) & 119.289 & \\
\hline B8469 & 10.0 & DD-12-2 & 11.200 & HR3D & $152,683.584$ & $573,819.657$ & SURV & ERC-GPS ID\#1056 (Sep 1996) & 117.030 & \\
\hline B8470 & 15.0 & DD-12-3 & 11.200 & HR3D & $152,683.696$ & $573,818.681$ & SURV & ERC-GPS ID\#1057 (Sep 1996) & 116.933 & \\
\hline B8471 & 21.0 & DD-12-4 & 11.200 & HR3D & $152,683.591$ & $573,820.559$ & SURV & ERC-GPS ID\#1058 (Sep 1996) & 117.154 & \\
\hline B8819 & 12.0 & DD-10-2 & 11.270 & HR3D & $152,788.072$ & $573,899.056$ & SURV & ERC-GPS ID\#1059 (Sep 1996) & 117.287 & \\
\hline B8820 & 17.0 & DD-10-3 & 11.270 & HR3D & $152,788.072$ & $573,899.056$ & SURV & ERC-GPS ID\#1059 (Sep 1996) & 117.287 & \\
\hline B8468 & 22.0 & DD-10-4 & 11.270 & HR3D & $152,788.072$ & $573,899.056$ & SURV & ERC-GPS ID\#1059 (Sep 1996) & 117.287 & \\
\hline C4318 & 6.8 & AT-D-5-M & 11.310 & HR3D & $152,848.206$ & $573,934.672$ & SURV & GPS ID1582 FHI-TimJohnson-March04 & 118.361 & \\
\hline $\mathrm{C} 4317$ & 8.8 & AT-D-5-D & 11.310 & HR3D & $152,850.597$ & $573,934.803$ & SURV & GPS ID1581 FHI-TimJohnson-March04 & 118.357 & \\
\hline B8821 & 11.2 & DD-08-2 & 11.340 & HR3D & $152,903.527$ & $573,940.057$ & EST & ERC-Project planning; L. Peterson (1997) & 118.000 & EST \\
\hline B8466 & 17.2 & DD-08-3 & 11.340 & HR3D & $152,903.527$ & $573,940.057$ & SURV & ERC-GPS ID\#3039 (Sep 1996) & 118.000 & EST \\
\hline B8467 & 22.3 & DD-08-4 & 11.340 & HR3D & $152,903.527$ & $573,940.057$ & EST & ERC-Project planning; L. Peterson (1997) & 118.000 & EST \\
\hline B8464 & 12.0 & DD-06-2 & 11.400 & HR3D & $153,015.374$ & $573,964.576$ & SURV & ERC-GPS ID\#1061 (Sep 1996) & 117.578 & \\
\hline B8465 & 16.0 & DD-06-3 & 11.400 & HR3D & $153,015.374$ & $573,964.576$ & SURV & ERC-GPS ID\#1061 (Sep 1996) & 117.578 & \\
\hline C6281 & 7.9 & C6281 & 11.564 & HR3D & $153,353.310$ & $574,056.820$ & SURV & AST Survey Data 1995-2008-MH-07509.xls & 116.700 & \\
\hline C6282 & 15.6 & C6282 & 11.564 & HR3D & $153,353.600$ & $574,057.530$ & SURV & AST Survey Data 1995-2008-MH-07509.xls & 116.770 & \\
\hline B8267 & 8.0 & $39-5$ & 11.620 & HR3D & $153,393.000$ & $574,035.000$ & EST & ERC-Project planning; L. Peterson (1997) & 117.000 & EST \\
\hline B8266 & 18.0 & $39-M$ & 11.620 & HR3D & $153,393.000$ & $574,035.000$ & EST & ERC-Project planning; L. Peterson (1997) & 117.000 & EST \\
\hline B8265 & 28.0 & 39-D & 11.620 & HR3D & $153,393.000$ & $574,035.000$ & EST & ERC-Project planning; L. Peterson (1997) & 117.000 & EST \\
\hline B8270 & 8.0 & $40-S$ & 11.980 & HR3D & $153,997.000$ & $574,238.000$ & EST & ERC-Project planning; L. Peterson (1997) & 116.800 & EST \\
\hline B8269 & 15.5 & $40-M$ & 11.980 & HR3D & $153,997.000$ & $574,238.000$ & EST & ERC-Project planning; L. Peterson (1997) & 116.800 & EST \\
\hline B8273 & 10.0 & 41-S & 13.100 & HR3D & $154,424.185$ & $575,515.614$ & SURV & ERC-GPS ID\#34 (Nov/Dec 1997) & 116.526 & \\
\hline B8272 & 15.0 & 41-M & 13.100 & HR3D & $154,423.723$ & $575,511.260$ & SURV & ERC-GPS ID\#32 (Nov/Dec 1997) & 116.509 & \\
\hline B8271 & 25.0 & 41-D & 13.100 & HR3D & $154,424.668$ & $575,512.511$ & SURV & ERC-GPS ID\#33 (Nov/Dec 1997) & 116.299 & \\
\hline C6284 & 9.7 & C6284 & 13.450 & HR3D & $154,363.630$ & $576,042.290$ & SURV & AST Survey Data 1995-2008-MH-07509.xls & 116.050 & \\
\hline C6285 & 14.6 & C6285 & 13.450 & HR3D & $154,364.310$ & $576,039.240$ & SURV & AST Survey Data 1995-2008-MH-07509.xls & 116.130 & \\
\hline C6286 & 34.0 & C6286 & 13.450 & HR3D & $154,364.500$ & $576,037.530$ & SURV & AST Survey Data 1995-2008-MH-07509.xls & 116.140 & \\
\hline B8276 & 10.0 & $42-S$ & 13.550 & HR3D & $154,320.154$ & $576,165.935$ & SURV & ERC-GPS ID\#37 (Nov/Dec 1997) & 116.376 & \\
\hline
\end{tabular}


Table 3.1. (contd)

\begin{tabular}{|c|c|c|c|c|c|c|c|c|c|c|}
\hline $\begin{array}{c}\text { Well } \\
\text { Identifier } \\
\text { (HWIS) }\end{array}$ & $\begin{array}{c}\text { Port } \\
\text { Depth } \\
\text { [to top of } \\
\text { opening] } \\
\text { (ft-bgs) }\end{array}$ & $\begin{array}{l}\text { Aquifer Tube } \\
\text { Name } \\
\text { (use in 2009) }\end{array}$ & $\begin{array}{l}\text { HRM } \\
\text { for Site } \\
\text { ("2k") }\end{array}$ & Segment $^{1}$ & $\begin{array}{c}\text { Northing } \\
\text { (m-NAD83) }\end{array}$ & $\begin{array}{c}\text { Easting } \\
\text { (m-NAD83) }\end{array}$ & $\begin{array}{l}\text { Coordinate } \\
\text { Quality }^{2}\end{array}$ & Coordinate Reference & $\begin{array}{c}\text { Ground } \\
\text { Elevation } \\
\text { (m-NAVD88) }\end{array}$ & $\begin{array}{l}\text { Elevation } \\
\text { Quality }^{2}\end{array}$ \\
\hline B8275 & 15.0 & $42-\mathrm{M}$ & 13.550 & HR3D & $154,320.454$ & $576,166.225$ & SURV & ERC-GPS ID\#36 (Nov/Dec 1997) & 116.339 & \\
\hline B8274 & 24.0 & $42-\mathrm{D}$ & 13.550 & HR3D & $154,320.018$ & $576,167.589$ & SURV & ERC-GPS ID\#35 (Nov/Dec 1997) & 116.273 & \\
\hline C5632 & 7.3 & C5632 & 13.722 & HR3H & $154,227.690$ & $576,389.250$ & SURV & AST Survey Data 1995-2008-MH-07509.xls & 115.620 & \\
\hline C5633 & 17.5 & C5633 & 13.722 & HR3H & $154,227.980$ & $576,390.600$ & SURV & AST Survey Data 1995-2008-MH-07509.xls & 115.520 & \\
\hline C5634 & 31.0 & C5634 & 13.722 & HR3H & $154,228.900$ & $576,391.430$ & SURV & AST Survey Data 1995-2008-MH-07509.xls & 115.320 & \\
\hline C5635 & 7.0 & C5635 & 13.791 & HR3H & $154,181.120$ & $576,478.810$ & SURV & AST Survey Data 1995-2008-MH-07509.xls & 115.420 & \\
\hline C5637 & 12.4 & C5637 & 13.791 & $\mathrm{HR} 3 \mathrm{H}$ & $154,178.300$ & $576,483.670$ & SURV & AST Survey Data 1995-2008-MH-07509.xls & 115.380 & \\
\hline C5636 & 15.6 & C5636 & 13.791 & HR3H & $154,179.430$ & $576,481.400$ & SURV & AST Survey Data 1995-2008-MH-07509.xls & 115.380 & \\
\hline C5638 & 5.7 & C5638 & 13.920 & HR3H & $154,070.380$ & $576,634.080$ & SURV & AST Survey Data 1995-2008-MH-07509.xls & 115.170 & \\
\hline B8278 & 7.5 & 43-M & 14.040 & HR3H & $153,963.570$ & $576,782.564$ & SURV & ERC-GPS ID\#38 (Nov/Dec 1997) & 116.411 & \\
\hline B8277 & 9.7 & 43-D & 14.040 & HR3H & $153,965.240$ & $576,779.961$ & SURV & ERC-GPS ID\#39 (Nov/Dec 1997) & 116.452 & \\
\hline C6287 & 6.5 & C6287 & 14.054 & HR3H & $153,965.550$ & $576,781.620$ & SURV & AST Survey Data 1995-2008-MH-07509.xls & 115.880 & \\
\hline $\mathrm{C6288}$ & 7.8 & C6288 & 14.054 & $\mathrm{HR} 3 \mathrm{H}$ & $153,964.050$ & $576,783.380$ & SURV & AST Survey Data 1995-2008-MH-07509.xls & 115.980 & \\
\hline C5641 & 4.7 & C5641 & 14.129 & HR3H & $153,916.620$ & $576,871.560$ & SURV & AST Survey Data 1995-2008-MH-07509.xls & 115.100 & \\
\hline C5644 & 6.4 & C5644 & 14.194 & $\mathrm{HR} 3 \mathrm{H}$ & $153,869.320$ & $576,960.870$ & SURV & AST Survey Data 1995-2008-MH-07509.xls & 114.820 & \\
\hline B8281 & 8.5 & $44-\mathrm{M}$ & 14.260 & HR3H & $153,810.532$ & $577,033.113$ & SURV & ERC-GPS ID\#40 (Nov/Dec 1997) & 116.085 & \\
\hline B8280 & 12.7 & $44-\mathrm{D}$ & 14.260 & HR3H & $153,811.744$ & $577,031.509$ & SURV & ERC-GPS ID\#41 (Nov/Dec 1997) & 116.114 & \\
\hline C5673 & 5.2 & C5673 & 14.365 & HR3H & $153,736.670$ & $577,157.450$ & SURV & AST Survey Data 1995-2008-MH-07509.xls & 114.760 & \\
\hline C5674 & 8.1 & C5674 & 14.365 & HR3H & $153,733.425$ & $577,156.226$ & SURV & AST Survey Data 1995-2008-MH-07509.xls & 114.760 & \\
\hline B8521 & & DH-1451-1 & 14.390 & $\mathrm{HR} 3 \mathrm{H}$ & $153,704.528$ & $577,182.865$ & SURV & ERC-GPS ID\#2039 (Sep 1996) & 115.982 & \\
\hline B8522 & & DH-1452-1 & 14.450 & HR3H & $153,661.681$ & $577,255.023$ & SURV & ERC-GPS ID\#2036 (Sep 1996) & 116.227 & \\
\hline C5676 & 5.4 & C5676 & 14.465 & $\mathrm{HR} 3 \mathrm{H}$ & $153,656.070$ & $577,276.260$ & SURV & AST Survey Data 1995-2008-MH-07509.xls & 115.300 & \\
\hline C5677 & 7.9 & C5677 & 14.465 & HR3H & $153,657.180$ & $577,277.330$ & SURV & AST Survey Data 1995-2008-MH-07509.xls & 114.850 & \\
\hline C5678 & 8.0 & C5678 & 14.465 & HR3H & $153,653.500$ & $577,278.650$ & SURV & AST Survey Data 1995-2008-MH-07509.xls & 115.370 & \\
\hline C5679 & 4.0 & C5679 & 14.548 & $\mathrm{HR} 3 \mathrm{H}$ & $153,593.400$ & $577,409.700$ & SURV & AST Survey Data 1995-2008-MH-07509.xls & 114.970 & \\
\hline C5680 & 11.9 & C5680 & 14.548 & HR3H & $153,593.790$ & $577,408.400$ & SURV & AST Survey Data 1995-2008-MH-07509.xls & 115.070 & \\
\hline C5681 & 13.2 & C5681 & 14.548 & HR3H & $153,594.580$ & $577,407.430$ & SURV & AST Survey Data 1995-2008-MH-07509.xls & 114.900 & \\
\hline B8285 & 8.0 & $45-\mathrm{S}$ & 14.660 & HR3H & $153,494.837$ & $577,600.791$ & SURV & PNNL-GPS (submeter) 3/17/04 & 116.000 & EST \\
\hline B8284 & 15.0 & $45-\mathrm{M}$ & 14.660 & HR3H & $153,494.837$ & $577,600.791$ & SURV & PNNL-GPS (submeter) 3/17/04 & 116.000 & EST \\
\hline B8283 & 23.0 & $45-\mathrm{D}$ & 14.660 & HR3H & $153,494.837$ & $577,600.791$ & SURV & PNNL-GPS (submeter) 3/17/04 & 116.000 & EST \\
\hline C5682 & 8.9 & C5682 & 14.836 & HR3H & $153,338.710$ & $577,699.790$ & SURV & AST Survey Data 1995-2008-MH-07509.xls & 115.340 & \\
\hline B8519 & 32.0 & DH-14-1 & 14.870 & HR3H & $153,374.820$ & $577,787.186$ & SURV & ERC-GPS ID\#2032 (Sep 1996) & 115.760 & \\
\hline B8520 & & DH-14-11 & 14.870 & HR3H & $153,377.955$ & $577,788.415$ & SURV & ERC-GPS ID\#2031 (Sep 1996) & 115.031 & \\
\hline C6290 & 8.6 & C6290 & 14.919 & HR3H & $153,266.010$ & $577,793.470$ & SURV & AST Survey Data 1995-2008-MH-07509.xls & 115.020 & \\
\hline C6291 & 13.0 & C6291 & 14.919 & $\mathrm{HR} 3 \mathrm{H}$ & $153,267.000$ & $577,792.390$ & SURV & \begin{tabular}{|l|l|} 
AST Survey Data 1995-2008-MH-07509.xls \\
\end{tabular} & 115.040 & \\
\hline
\end{tabular}


Table 3.1. (contd)

\begin{tabular}{|c|c|c|c|c|c|c|c|c|c|c|}
\hline $\begin{array}{c}\text { Well } \\
\text { Identifier } \\
\text { (HWIS) }\end{array}$ & $\begin{array}{c}\text { Port } \\
\text { Depth } \\
\text { [to top of } \\
\text { opening] } \\
\text { (ft-bgs) }\end{array}$ & $\begin{array}{l}\text { Aquifer Tube } \\
\text { Name } \\
\text { (use in 2009) }\end{array}$ & $\begin{array}{c}\text { HRM } \\
\text { for Site } \\
\text { ("2k") }\end{array}$ & Segment $^{1}$ & $\begin{array}{c}\text { Northing } \\
\text { (m-NAD83) }\end{array}$ & $\begin{array}{c}\text { Easting } \\
\text { (m-NAD83) }\end{array}$ & $\begin{array}{c}\text { Coordinate } \\
\text { Quality }^{2}\end{array}$ & Coordinate Reference & $\begin{array}{c}\text { Ground } \\
\text { Elevation } \\
\text { (m-NAVD88) }\end{array}$ & $\begin{array}{l}\text { Elevation } \\
\text { Quality }^{2}\end{array}$ \\
\hline B8286 & 10.5 & $46-\mathrm{D}$ & 15.120 & $\mathrm{HR} 3 \mathrm{H}$ & $152,999.261$ & $578,031.495$ & SURV & ERC-GPS ID\#42 (Nov/Dec 1997) & 115.347 & \\
\hline C6293 & 7.9 & C6293 & 15.129 & $\mathrm{HR} 3 \mathrm{H}$ & $152,996.700$ & $578,035.060$ & SURV & AST Survey Data 1995-2008-MH-07509.xls & 115.080 & \\
\hline C4321 & 6.2 & AT-H-1-S & 15.160 & $\mathrm{HR} 3 \mathrm{H}$ & $152,957.484$ & $578,070.238$ & SURV & GPS ID1587 FHI-TimJohnson-March04 & 115.233 & \\
\hline C4320 & 11.0 & AT-H-1-M & 15.160 & $\mathrm{HR} 3 \mathrm{H}$ & $152,955.059$ & $578,068.733$ & SURV & GPS ID1588 FHI-TimJohnson-March04 & 115.542 & \\
\hline C4319 & 12.8 & AT-H-1-D & 15.160 & $\mathrm{HR} 3 \mathrm{H}$ & $152,954.335$ & $578,069.144$ & SURV & GPS ID1589 FHI-TimJohnson-March04 & 115.529 & \\
\hline C4324 & 5.3 & AT-H-2-S & 15.200 & $\mathrm{HR} 3 \mathrm{H}$ & $152,895.191$ & $578,110.719$ & SURV & GPS ID1591 FHI-TimJohnson-March04 & 115.470 & \\
\hline C4323 & 9.2 & AT-H-2-M & 15.200 & $\mathrm{HR} 3 \mathrm{H}$ & $152,893.943$ & $578,111.797$ & SURV & GPS ID1592 FHI-TimJohnson-March04 & 115.450 & \\
\hline C4322 & 12.0 & AT-H-2-D & 15.200 & $\mathrm{HR} 3 \mathrm{H}$ & $152,896.440$ & $578,109.838$ & SURV & GPS ID1590 FHI-TimJohnson-March04 & 115.442 & \\
\hline C4326 & 5.3 & AT-H-3-S & 15.230 & $\mathrm{HR} 3 \mathrm{H}$ & $152,848.945$ & $578,141.784$ & SURV & GPS ID1594 FHI-TimJohnson-March04 & 115.428 & \\
\hline C4325 & 7.3 & AT-H-3-D & 15.230 & $\mathrm{HR} 3 \mathrm{H}$ & $152,853.072$ & $578,142.216$ & SURV & GPS ID1593 FHI-TimJohnson-March04 & 115.309 & \\
\hline B8290 & 8.0 & $47-\mathrm{M}$ & 15.300 & $\mathrm{HR} 3 \mathrm{H}$ & $152,743.533$ & $578,193.919$ & SURV & ERC-GPS ID\#22 (Nov/Dec 1997) & 115.765 & \\
\hline B8289 & 14.5 & 47-D & 15.300 & $\mathrm{HR} 3 \mathrm{H}$ & $152,744.421$ & $578,193.394$ & SURV & ERC-GPS ID\#21 (Nov/Dec 1997) & 115.856 & \\
\hline C6296 & 8.0 & C6296 & 15.411 & $\mathrm{HR} 3 \mathrm{H}$ & $152,574.800$ & $578,314.840$ & SURV & AST Survey Data 1995-2008-MH-07509.xls & 115.130 & \\
\hline C6297 & 13.1 & C6297 & 15.411 & $\mathrm{HR} 3 \mathrm{H}$ & $152,575.130$ & $578,313.220$ & SURV & AST Survey Data 1995-2008-MH-07509.xls & 115.160 & \\
\hline B8523 & 4.0 & $\mathrm{DH}-22-1$ & 15.500 & $\mathrm{HR} 3 \mathrm{H}$ & $152,468.304$ & $578,460.331$ & SURV & ERC-GPS ID\#2000 (Sep 1996) & 114.224 & \\
\hline B8524 & 8.0 & $\mathrm{DH}-22-2$ & 15.500 & $\mathrm{HR} 3 \mathrm{H}$ & $152,468.304$ & $578,460.331$ & SURV & ERC-GPS ID\#2000 (Sep 1996) & 114.224 & \\
\hline B8525 & 13.5 & $\mathrm{DH}-22-3$ & 15.500 & $\mathrm{HR} 3 \mathrm{H}$ & $152,468.304$ & $578,460.331$ & SURV & ERC-GPS ID\#2000 (Sep 1996) & 114.224 & \\
\hline B8294 & 9.0 & $48-S$ & 15.520 & $\mathrm{HR} 3 \mathrm{H}$ & $152,420.795$ & $578,465.520$ & SURV & ERC-GPS ID\#24 (Nov/Dec 1997) & 116.348 & \\
\hline B8293 & 17.0 & $48-\mathrm{M}$ & 15.520 & $\mathrm{HR} 3 \mathrm{H}$ & $152,420.795$ & $578,465.520$ & SURV & ERC-GPS ID\#24 (Nov/Dec 1997) & 116.348 & \\
\hline B8292 & 25.0 & 48-D & 15.520 & HR3H & $152,420.795$ & $578,465.520$ & SURV & ERC-GPS ID\#24 (Nov/Dec 1997) & 116.348 & \\
\hline C6299 & 8.1 & C6299 & 15.618 & HR3H & $152,323.620$ & $578,585.360$ & SURV & AST Survey Data 1995-2008-MH-07509.xls & 115.150 & \\
\hline $\mathrm{C} 6300$ & 12.6 & $\mathrm{C} 6300$ & 15.618 & $\mathrm{HR} 3 \mathrm{H}$ & $152,324.650$ & $578,585.170$ & SURV & AST Survey Data 1995-2008-MH-07509.xls & 115.030 & \\
\hline C6301 & 17.3 & C6301 & 15.618 & HR3H & $152,323.270$ & $578,583.540$ & SURV & AST Survey Data 1995-2008-MH-07509.xls & 115.350 & \\
\hline B8297 & 8.5 & $49-S$ & 15.680 & $\mathrm{HR} 3 \mathrm{H}$ & $152,232.848$ & $578,699.729$ & SURV & ERC-GPS ID\#26 (Nov/Dec 1997) & 115.985 & \\
\hline B8296 & 17.5 & $49-\mathrm{M}$ & 15.680 & $\mathrm{HR} 3 \mathrm{H}$ & $152,232.848$ & $578,699.729$ & SURV & ERC-GPS ID\#26 (Nov/Dec 1997) & 115.985 & \\
\hline B8295 & 25.5 & 49-D & 15.680 & $\mathrm{HR} 3 \mathrm{H}$ & $152,232.848$ & $578,699.729$ & SURV & ERC-GPS ID\#26 (Nov/Dec 1997) & 115.985 & \\
\hline B8300 & 8.5 & $50-\mathrm{s}$ & 15.710 & $\mathrm{HR} 3 \mathrm{H}$ & $152,118.154$ & $578,611.792$ & SURV & ERC-GPS ID\#27 (Nov/Dec 1997) & 117.184 & \\
\hline B8299 & 17.5 & $50-\mathrm{M}$ & 15.710 & $\mathrm{HR} 3 \mathrm{H}$ & $152,118.154$ & $578,611.792$ & SURV & ERC-GPS ID\#27 (Nov/Dec 1997) & 117.184 & \\
\hline B8298 & 26.5 & $50-\mathrm{D}$ & 15.710 & $\mathrm{HR} 3 \mathrm{H}$ & $152,118.154$ & $578,611.792$ & SURV & ERC-GPS ID\#45 (Nov/Dec 1997) & 117.184 & \\
\hline B8303 & 9.5 & $51-S$ & 15.890 & $\mathrm{HR} 3 \mathrm{H}$ & $151,843.755$ & $578,779.254$ & SURV & ERC-GPS ID\#46 (Nov/Dec 1997) & 116.760 & \\
\hline B8302 & 17.5 & $51-\mathrm{M}$ & 15.890 & HR3H & $151,843.755$ & $578,779.254$ & SURV & ERC-GPS ID\#46 (Nov/Dec 1997) & 116.760 & \\
\hline B8301 & 25.5 & 51-D & 15.890 & $\mathrm{HR} 3 \mathrm{H}$ & $151,843.755$ & $578,779.254$ & SURV & ERC-GPS ID\#46 (Nov/Dec 1997) & 116.760 & \\
\hline B8306 & 7.0 & $52-\mathrm{S}$ & 16.100 & $\mathrm{HR} 3 \mathrm{H}$ & $151,586.176$ & $578,919.484$ & SURV & ERC-GPS ID\#47 (Nov/Dec 1997) & 115.996 & \\
\hline B8305 & 15.0 & $52-M$ & 16.100 & $\mathrm{HR} 3 \mathrm{H}$ & $151,586.176$ & $578,919.484$ & SURV & ERC-GPS ID\#47 (Nov/Dec 1997) & 115.996 & \\
\hline B8304 & 24.0 & $52-\mathrm{D}$ & 16.100 & $\mathrm{HR} 3 \mathrm{H}$ & $151,586.176$ & $578,919.484$ & SURV & ERC-GPS ID\#47 (Nov/Dec 1997) & 115.996 & \\
\hline
\end{tabular}


Table 3.1. (contd)

\begin{tabular}{|c|c|c|c|c|c|c|c|c|c|c|}
\hline $\begin{array}{c}\text { Well } \\
\text { Identifier } \\
\text { (HWIS) }\end{array}$ & $\begin{array}{c}\text { Port } \\
\text { Depth } \\
\text { [to top of } \\
\text { opening] } \\
\text { (ft-bgs) }\end{array}$ & $\begin{array}{c}\text { Aquifer Tube } \\
\text { Name } \\
\text { (use in 2009) }\end{array}$ & $\begin{array}{c}\text { HRM } \\
\text { for Site } \\
\text { ("2k") }\end{array}$ & Segment $^{1}$ & $\begin{array}{c}\text { Northing } \\
\text { (m-NAD83) }\end{array}$ & $\begin{array}{c}\text { Easting } \\
\text { (m-NAD83) }\end{array}$ & $\begin{array}{c}\text { Coordinate } \\
\text { Quality }^{2}\end{array}$ & Coordinate Reference & $\begin{array}{c}\text { Ground } \\
\text { Elevation } \\
\text { (m-NAVD88) }\end{array}$ & $\begin{array}{r}\text { Elevation } \\
\text { Quality }^{2} \\
\end{array}$ \\
\hline B8309 & 8.0 & $53-5$ & 16.370 & $\mathrm{HR} 3 \mathrm{H}$ & $151,531.727$ & $579,510.574$ & SURV & ERC-GPS ID\#49 (Nov/Dec 1997) & 115.334 & \\
\hline B8308 & 17.0 & $53-\mathrm{M}$ & 16.370 & $\mathrm{HR} 3 \mathrm{H}$ & $151,531.727$ & $579,510.574$ & SURV & ERC-GPS ID\#48 (Nov/Dec 1997) & 115.334 & \\
\hline B8307 & 26.0 & 53-D & 16.370 & HR3H & $151,531.413$ & $579,510.188$ & SURV & ERC-GPS ID\#49 (Nov/Dec 1997) & 115.356 & \\
\hline B8312 & 7.5 & $54-S$ & 16.400 & HR3H & $151,231.944$ & $579,033.518$ & SURV & ERC-GPS ID\#50 (Nov/Dec 1997) & 116.000 & \\
\hline B8311 & 17.0 & 54-M & 16.400 & HR3H & $151,231.944$ & $579,033.518$ & SURV & ERC-GPS ID\#50 (Nov/Dec 1997) & 116.000 & \\
\hline B8310 & 26.0 & 54-D & 16.400 & $\mathrm{HR} 3 \mathrm{H}$ & $151,231.944$ & $579,033.518$ & SURV & ERC-GPS ID\#50 (Nov/Dec 1997) & 116.000 & \\
\hline B8315 & 10.0 & $55-S$ & 16.670 & HR3H & $150,891.146$ & $579,134.582$ & SURV & ERC-GPS ID\#79 (Nov/Dec 1997) & 115.295 & \\
\hline B8314 & 18.0 & $55-\mathrm{M}$ & 16.670 & $\mathrm{HR} 3 \mathrm{H}$ & $150,891.146$ & $579,134.582$ & SURV & ERC-GPS ID\#79 (Nov/Dec 1997) & 115.295 & \\
\hline B8313 & 26.0 & $55-\mathrm{D}$ & 16.670 & $\mathrm{HR} 3 \mathrm{H}$ & $150,891.368$ & $579,135.286$ & SURV & ERC-GPS ID\#78 (Nov/Dec 1997) & 115.251 & \\
\hline B8321 & 7.0 & $57-S$ & 17.100 & HR3H & $150,525.000$ & $580,101.000$ & EST & ERC-Project planning; L. Peterson (1997) & 115.000 & EST \\
\hline $\mathrm{B} 8320$ & 18.0 & 57-M & 17.100 & $\mathrm{HR} 3 \mathrm{H}$ & $150,525.000$ & $580,101.000$ & EST & ERC-Project planning; L. Peterson (1997) & 115.000 & EST \\
\hline $\mathrm{B} 8319$ & 29.0 & $57-\mathrm{D}$ & 17.100 & $\mathrm{HR} 3 \mathrm{H}$ & $150,525.000$ & $580,101.000$ & EST & ERC-Project planning; L. Peterson (1997) & 115.000 & EST \\
\hline B8324 & 11.0 & $58-S$ & 17.160 & $\mathrm{HR} 3 \mathrm{H}$ & $150,232.255$ & $579,310.627$ & SURV & ERC-GPS ID\#51 (Nov/Dec 1997) & 114.929 & \\
\hline B8323 & 19.5 & $58-\mathrm{M}$ & 17.160 & HR3H & $150,230.317$ & $579,311.429$ & SURV & ERC-GPS ID\#80 (Nov/Dec 1997) & 114.880 & \\
\hline $\mathrm{B} 8322$ & 26.5 & 58-D & 17.160 & $\mathrm{HR} 3 \mathrm{H}$ & $150,233.170$ & $579,310.118$ & SURV & ERC-GPS ID\#52 (Nov/Dec 1997) & 114.858 & \\
\hline $\mathrm{B} 8327$ & 11.0 & $59-S$ & 17.380 & $\mathrm{HR} 3 \mathrm{H}$ & $149,928.523$ & $579,420.404$ & SURV & ERC-GPS ID\#83 (Nov/Dec 1997) & 114.857 & \\
\hline $\mathrm{B} 8326$ & 16.5 & $59-\mathrm{M}$ & 17.380 & $\mathrm{HR} 3 \mathrm{H}$ & $149,930.907$ & $579,420.300$ & SURV & ERC-GPS ID\#82 (Nov/Dec 1997) & 114.860 & \\
\hline B8325 & 23.0 & 59-D & 17.380 & $\mathrm{HR} 3 \mathrm{H}$ & $149,930.079$ & $579,420.305$ & SURV & ERC-GPS ID\#81 (Nov/Dec 1997) & 114.963 & \\
\hline B8330 & 8.5 & $60-S$ & 17.740 & HR3H & $149,392.000$ & $579,638.000$ & EST & ERC-Project planning; L. Peterson (1997) & 115.000 & EST \\
\hline B8329 & 17.5 & $60-\mathrm{M}$ & 17.740 & $\mathrm{HR} 3 \mathrm{H}$ & $149,392.000$ & $579,638.000$ & EST & ERC-Project planning; L. Peterson (1997) & 115.000 & EST \\
\hline B8328 & 26.5 & $60-\mathrm{D}$ & 17.740 & $\mathrm{HR} 3 \mathrm{H}$ & $149,392.000$ & $579,638.000$ & EST & ERC-Project planning; L. Peterson (1997) & 115.000 & EST \\
\hline $\mathrm{B} 8333$ & 8.5 & $61-\mathrm{S}$ & 18.100 & FR3 & $148,950.427$ & $580,000.071$ & SURV & ERC-GPS ID\#86 (Nov/Dec 1997) & 115.079 & \\
\hline B8332 & 15.5 & 61-M & 18.100 & FR3 & $148,949.889$ & $580,001.323$ & SURV & ERC-GPS ID\#85 (Nov/Dec 1997) & 115.017 & \\
\hline B8331 & 24.0 & 61-D & 18.100 & FR3 & $148,949.718$ & $580,002.501$ & SURV & ERC-GPS ID\#84 (Nov/Dec 1997) & 115.001 & \\
\hline B8336 & 8.0 & $62-S$ & 18.400 & FR3 & $148,585.876$ & $580,388.292$ & SURV & ERC-GPS ID\#53 (Nov/Dec 1997) & 115.092 & \\
\hline B8335 & 18.0 & $62-M$ & 18.400 & FR3 & $148,586.349$ & $580,387.648$ & SURV & ERC-GPS ID\#54 (Nov/Dec 1997) & 115.246 & \\
\hline B8334 & 28.0 & 62-D & 18.400 & FR3 & $148,585.095$ & $580,389.375$ & SURV & ERC-GPS ID\#19 (Nov/Dec 1997) & 115.395 & \\
\hline B8339 & 10.0 & $63-S$ & 18.810 & FR3 & $148,174.193$ & $580,945.002$ & SURV & ERC-GPS ID\#16 (Nov/Dec 1997) & 115.055 & \\
\hline B8338 & 16.0 & $63-\mathrm{M}$ & 18.810 & FR3 & $148,174.517$ & $580,944.736$ & SURV & ERC-GPS ID\#17 (Nov/Dec 1997) & 115.122 & \\
\hline B8337 & 23.0 & 63-D & 18.810 & FR3 & $148,175.400$ & $580,943.236$ & SURV & ERC-GPS ID\#18 (Nov/Dec 1997) & 115.077 & \\
\hline C6302 & 8.5 & C6302 & 18.815 & FR3 & $148,180.160$ & $580,940.000$ & SURV & AST Survey Data 1995-2008-MH-07509.xls & 113.850 & \\
\hline C6303 & 13.3 & C6303 & 18.815 & FR3 & $148,180.040$ & $580,942.300$ & SURV & AST Survey Data 1995-2008-MH-07509.xls & 113.500 & \\
\hline B8342 & 7.5 & $64-\mathrm{S}$ & 18.940 & FR3 & $148,037.975$ & $581,118.756$ & SURV & ERC-GPS ID\#15 (Nov/Dec 1997) & 115.386 & \\
\hline B8341 & 17.0 & $64-M$ & 18.940 & FR3 & $148,037.975$ & $581,118.756$ & SURV & PNNL Recon, Trimble sub-meter; 4/7/04 & 115.386 & EST \\
\hline B8340 & 27.0 & 64-D & 18.940 & FR3 & $148,037.975$ & $581,118.756$ & SURV & PNNL Recon, Trimble sub-meter; 4/7/04 & 115.386 & EST \\
\hline
\end{tabular}


Table 3.1. (contd)

\begin{tabular}{|c|c|c|c|c|c|c|c|c|c|c|}
\hline $\begin{array}{c}\text { Well } \\
\text { Identifier } \\
\text { (HWIS) }\end{array}$ & $\begin{array}{c}\text { Port } \\
\text { Depth } \\
\text { [to top of } \\
\text { opening] } \\
\text { (ft-bgs) }\end{array}$ & $\begin{array}{l}\text { Aquifer Tube } \\
\quad \text { Name } \\
\text { (use in 2009) }\end{array}$ & $\begin{array}{c}\text { HRM } \\
\text { for Site } \\
\text { ("2k") }\end{array}$ & Segment $^{1}$ & $\begin{array}{c}\begin{array}{c}\text { Northing } \\
\text { (m-NAD83) }\end{array} \\
\end{array}$ & $\begin{array}{c}\text { Easting } \\
\text { (m-NAD83) }\end{array}$ & $\begin{array}{c}\text { Coordinate } \\
\text { Quality }^{2}\end{array}$ & Coordinate Reference & $\begin{array}{c}\text { Ground } \\
\text { Elevation } \\
\text { (m-NAVD88) }\end{array}$ & $\begin{array}{c}\begin{array}{c}\text { Elevation } \\
\text { Quality }^{2}\end{array} \\
\end{array}$ \\
\hline C6305 & 8.7 & C6305 & 19.049 & FR3 & $147,922.660$ & $581,252.270$ & SURV & AST Survey Data 1995-2008-MH-07509.xls & 113.790 & \\
\hline C6306 & 14.3 & C6306 & 19.049 & FR3 & $147,924.000$ & $581,251.350$ & SURV & AST Survey Data 1995-2008-MH-07509.xls & 113.760 & \\
\hline C6307 & 16.4 & C6307 & 19.049 & FR3 & $147,926.320$ & $581,250.540$ & SURV & AST Survey Data 1995-2008-MH-07509.xls & 113.590 & \\
\hline B8345 & 8.5 & $65-S$ & 19.100 & FR3 & $147,864.503$ & $581,297.412$ & SURV & ERC-GPS ID\#13 (Nov/Dec 1997) & 115.367 & \\
\hline B8344 & 16.0 & $65-\mathrm{M}$ & 19.100 & FR3 & $147,866.273$ & $581,295.747$ & SURV & ERC-GPS ID\#13 (Nov/Dec 1997) & 115.502 & \\
\hline B8343 & 27.0 & 65-D & 19.100 & FR3 & $147,866.273$ & $581,295.747$ & SURV & ERC-GPS ID\#14 (Nov/Dec 1997) & 115.502 & \\
\hline C6308 & 9.4 & C6308 & 19.105 & FR3 & $147,871.210$ & $581,296.370$ & SURV & AST Survey Data 1995-2008-MH-07509.xls & 113.810 & \\
\hline C6309 & 16.2 & C6309 & 19.105 & FR3 & $147,874.110$ & $581,294.050$ & SURV & AST Survey Data 1995-2008-MH-07509.xls & 113.810 & \\
\hline C6311 & 9.7 & C6311 & 19.141 & FR3 & $147,821.260$ & $581,332.970$ & SURV & AST Survey Data 1995-2008-MH-07509.xls & 113.770 & \\
\hline C6312 & 15.9 & C6312 & 19.141 & FR3 & $147,823.560$ & $581,331.180$ & SURV & AST Survey Data 1995-2008-MH-07509.xls & 113.710 & \\
\hline C6314 & 11.6 & C6314 & 19.179 & FR3 & $147,760.530$ & $581,354.660$ & SURV & AST Survey Data 1995-2008-MH-07509.xls & 114.580 & \\
\hline C6315 & 18.4 & C6315 & 19.179 & FR3 & $147,761.410$ & $581,354.490$ & SURV & AST Survey Data 1995-2008-MH-07509.xls & 114.610 & \\
\hline C6316 & 27.3 & C6316 & 19.179 & FR3 & $147,759.410$ & $581,354.760$ & SURV & AST Survey Data 1995-2008-MH-07509.xls & 114.650 & \\
\hline C4391 & 10.3 & AT-F-1-S & 19.220 & FR3 & $147,707.333$ & $581,378.715$ & SURV & GPS ID1630 FHI-TimJohnson-March04 & 114.798 & \\
\hline $\mathrm{C} 4390$ & 18.1 & AT-F-1-M & 19.220 & FR3 & $147,708.405$ & $581,378.347$ & SURV & GPS ID1631 FHI-TimJohnson-March04 & 114.738 & \\
\hline C4389 & 26.1 & AT-F-1-D & 19.220 & FR3 & $147,709.395$ & $581,377.937$ & SURV & GPS ID1632 FHI-TimJohnson-March04 & 114.734 & \\
\hline B8348 & 10.0 & $66-5$ & 19.370 & FR3 & $147,541.818$ & $581,475.927$ & SURV & ERC-GPS ID\#12 (Nov/Dec 1997) & 114.421 & \\
\hline B8347 & 19.2 & $66-\mathrm{M}$ & 19.370 & FR3 & $147,541.818$ & $581,475.927$ & SURV & ERC-GPS ID\#12 (Nov/Dec 1997) & 114.421 & \\
\hline B8346 & 28.1 & 66-D & 19.370 & FR3 & $147,541.818$ & $581,475.927$ & SURV & ERC-GPS ID\#12 (Nov/Dec 1997) & 114.421 & \\
\hline B8351 & 10.0 & $67-\mathrm{S}$ & 19.580 & FR3 & $147,291.431$ & $581,616.352$ & SURV & ERC-GPS ID\#11 (Nov/Dec 1997) & 114.902 & \\
\hline B8350 & 20.0 & $67-\mathrm{M}$ & 19.580 & FR3 & $147,291.431$ & $581,616.352$ & SURV & ERC-GPS ID\#11 (Nov/Dec 1997) & 114.902 & \\
\hline B8349 & 30.0 & 67-D & 19.580 & FR3 & $147,291.431$ & $581,616.352$ & SURV & ERC-GPS ID\#11 (Nov/Dec 1997) & 114.902 & \\
\hline B8354 & 10.5 & $68-S$ & 19.760 & FR3 & $147,085.176$ & $581,758.431$ & SURV & ERC-GPS ID\#10 (Nov/Dec 1997) & 115.459 & \\
\hline B8353 & 18.3 & $68-\mathrm{M}$ & 19.760 & FR3 & $147,085.176$ & $581,758.431$ & SURV & ERC-GPS ID\#10 (Nov/Dec 1997) & 115.459 & \\
\hline B8352 & 25.0 & 68-D & 19.760 & FR3 & $147,085.176$ & $581,758.431$ & SURV & ERC-GPS ID\#10 (Nov/Dec 1997) & 115.459 & \\
\hline B8356 & 15.0 & $69-M$ & 19.800 & FR3 & $147,168.000$ & $582,017.000$ & EST & ERC-Project planning; L. Peterson (1997) & 116.000 & EST \\
\hline B8355 & 31.0 & 69-D & 19.800 & FR3 & $147,168.000$ & $582,017.000$ & EST & ERC-Project planning; L. Peterson (1997) & 116.000 & EST \\
\hline B8360 & 17.0 & $70-S$ & 19.950 & FR3 & $146,843.106$ & $581,902.543$ & SURV & ERC-GPS ID\#55 (Nov/Dec 1997) & 116.720 & \\
\hline B8359 & 24.0 & $70-\mathrm{M}$ & 19.950 & FR3 & $146,843.106$ & $581,902.543$ & SURV & ERC-GPS ID\#55 (Nov/Dec 1997) & 116.720 & \\
\hline B8358 & 31.0 & 70-D & 19.950 & FR3 & $146,843.106$ & $581,902.543$ & SURV & ERC-GPS ID\#55 (Nov/Dec 1997) & 116.720 & \\
\hline C4394 & 7.5 & AT-F-2-S & 20.110 & FR3 & $146,621.604$ & $581,956.970$ & SURV & GPS ID1628 FHI-TimJohnson-March04 & 112.703 & \\
\hline C4393 & 13.6 & AT-F-2-M & 20.110 & FR3 & $146,619.127$ & $581,957.953$ & SURV & GPS ID1629 FHI-TimJohnson-March04 & 112.633 & \\
\hline C4392 & 19.3 & AT-F-2-D & 20.110 & FR3 & $146,622.707$ & $581,956.315$ & SURV & GPS ID1627 FHI-TimJohnson-March04 & 112.607 & \\
\hline B8361 & 7.5 & 71-D & 20.260 & FR3 & $146,480.300$ & $582,009.900$ & SURV & PNNL Recon, Trimble sub-meter; 4/7/04 & 112.700 & EST \\
\hline C4385 & 6.0 & AT-F-3-S & 20.420 & FR3 & $146,285.494$ & $582,089.124$ & SURV & GPS ID1625 FHI-TimJohnson-March04 & 112.628 & \\
\hline
\end{tabular}


Table 3.1. (contd)

\begin{tabular}{|c|c|c|c|c|c|c|c|c|c|c|}
\hline $\begin{array}{c}\text { Well } \\
\text { Identifier } \\
\text { (HWIS) }\end{array}$ & $\begin{array}{c}\text { Port } \\
\text { Depth } \\
\text { [to top of } \\
\text { opening] } \\
\text { (ft-bgs) }\end{array}$ & $\begin{array}{c}\text { Aquifer Tube } \\
\text { Name } \\
\text { (use in 2009) }\end{array}$ & $\begin{array}{l}\text { HRM } \\
\text { for Site } \\
\text { ("2k") }\end{array}$ & Segment $^{1}$ & $\begin{array}{c}\text { Northing } \\
\text { (m-NAD83) }\end{array}$ & $\begin{array}{c}\text { Easting } \\
\text { (m-NAD83) }\end{array}$ & $\begin{array}{c}\text { Coordinate } \\
\text { Quality }^{2}\end{array}$ & Coordinate Reference & $\begin{array}{c}\text { Ground } \\
\text { Elevation } \\
\text { (m-NAVD88) }\end{array}$ & $\begin{array}{l}\text { Elevation } \\
\text { Quality }^{2}\end{array}$ \\
\hline C4384 & 10.8 & AT-F-3-M & 20.420 & FR3 & $146,286.839$ & $582,088.405$ & SURV & GPS ID1626 FHI-TimJohnson-March04 & 112.667 & \\
\hline C4383 & 14.1 & AT-F-3-D & 20.420 & FR3 & $146,284.025$ & $582,089.480$ & SURV & GPS ID1624 FHI-TimJohnson-March04 & 112.696 & \\
\hline B8366 & 9.5 & $72-S$ & 20.670 & FR3 & $146,067.007$ & $582,223.666$ & SURV & ERC-GPS ID\#58 (Nov/Dec 1997) & 112.400 & EST \\
\hline B8365 & 18.0 & 72-M & 20.670 & FR3 & $146,067.252$ & $582,224.292$ & SURV & ERC-GPS ID\#57 (Nov/Dec 1997) & 112.400 & EST \\
\hline B8364 & 28.0 & 72-D & 20.670 & FR3 & $146,066.634$ & $582,224.473$ & SURV & ERC-GPS ID\#56 (Nov/Dec 1997) & 112.400 & EST \\
\hline B8369 & 10.5 & 73-S & 20.810 & FR3 & $145,989.728$ & $582,424.818$ & SURV & ERC-GPS ID\#61 (Nov/Dec 1997) & 112.200 & EST \\
\hline B8368 & 19.0 & 73-M & 20.810 & FR3 & $145,989.251$ & $582,424.473$ & SURV & ERC-GPS ID\#60 (Nov/Dec 1997) & 112.200 & EST \\
\hline B8367 & 27.0 & 73-D & 20.810 & FR3 & $145,988.225$ & $582,424.378$ & SURV & ERC-GPS ID\#59 (Nov/Dec 1997) & 112.200 & EST \\
\hline C4388 & 7.2 & AT-F-4-S & 21.000 & FR3 & $145,787.132$ & $582,491.278$ & SURV & GPS ID1623 FHI-TimJohnson-March04 & 112.106 & \\
\hline C4387 & 18.0 & AT-F-4-M & 21.000 & FR3 & $145,787.683$ & $582,491.931$ & SURV & GPS ID1622 FHI-TimJohnson-March04 & 112.118 & \\
\hline C4386 & 32.3 & AT-F-4-D & 21.000 & FR3 & $145,788.865$ & $582,492.808$ & SURV & GPS ID1621 FHI-TimJohnson-March04 & 112.130 & \\
\hline B8372 & 11.0 & $74-S$ & 21.160 & FR3 & $145,609.940$ & $582,599.498$ & SURV & ERC-GPS ID\#64 (Nov/Dec 1997) & 112.200 & EST \\
\hline B8371 & 17.0 & $74-M$ & 21.160 & FR3 & $145,609.471$ & $582,599.881$ & SURV & ERC-GPS ID\#63 (Nov/Dec 1997) & 112.200 & EST \\
\hline B8370 & 29.0 & 74-D & 21.160 & FR3 & $145,608.435$ & $582,600.480$ & SURV & ERC-GPS ID\#62 (Nov/Dec 1997) & 112.200 & EST \\
\hline B8375 & 11.0 & $75-S$ & 21.490 & FR3 & $145,287.068$ & $582,790.915$ & SURV & ERC-GPS ID\#67 (Nov/Dec 1997) & 111.700 & EST \\
\hline B8374 & 19.0 & $75-M$ & 21.490 & FR3 & $145,285.862$ & $582,792.893$ & SURV & ERC-GPS ID\#66 (Nov/Dec 1997) & 111.700 & EST \\
\hline B8373 & 27.0 & $75-\mathrm{D}$ & 21.490 & FR3 & $145,283.988$ & $582,793.490$ & SURV & ERC-GPS ID\#65 (Nov/Dec 1997) & 111.700 & EST \\
\hline B8378 & 11.0 & $76-\mathrm{S}$ & 21.680 & FR3 & $145,089.114$ & $582,877.989$ & SURV & ERC-GPS ID\#70 (Nov/Dec 1997) & 111.600 & EST \\
\hline B8377 & 19.0 & $76-\mathrm{M}$ & 21.680 & FR3 & $145,088.849$ & $582,878.733$ & SURV & ERC-GPS ID\#69 (Nov/Dec 1997) & 111.600 & EST \\
\hline B8376 & 25.0 & $76-\mathrm{D}$ & 21.680 & FR3 & $145,087.325$ & $582,878.748$ & SURV & ERC-GPS ID\#68 (Nov/Dec 1997) & 111.600 & EST \\
\hline B8381 & 8.5 & $77-S$ & 21.860 & FR3 & $144,889.256$ & $582,957.215$ & SURV & ERC-GPS ID\#71 (Nov/Dec 1997) & 111.539 & \\
\hline B8380 & 16.5 & 77-M & 21.860 & FR3 & $144,889.256$ & $582,957.215$ & SURV & ERC-GPS ID\#71 (Nov/Dec 1997) & 111.539 & \\
\hline B8379 & 24.5 & 77-D & 21.860 & FR3 & $144,889.256$ & $582,957.215$ & SURV & ERC-GPS ID\#71 (Nov/Dec 1997) & 111.539 & \\
\hline B8384 & 8.0 & $78-S$ & 22.300 & FR3 & $144,325.675$ & $583,152.559$ & SURV & ERC-GPS ID\#72 (Nov/Dec 1997) & 111.346 & \\
\hline B8383 & 16.0 & $78-\mathrm{M}$ & 22.300 & FR3 & $144,325.675$ & $583,152.559$ & SURV & ERC-GPS ID\#72 (Nov/Dec 1997) & 111.346 & \\
\hline B8382 & 24.0 & $78-\mathrm{D}$ & 22.300 & FR3 & $144,325.675$ & $583,152.559$ & SURV & ERC-GPS ID\#72 (Nov/Dec 1997) & 111.346 & \\
\hline B8390 & 5.0 & $80-5$ & 23.100 & HTS & $143,178.792$ & $583,509.431$ & SURV & ERC-GPS ID\#74 (Nov/Dec 1997) & 111.826 & \\
\hline B8389 & 15.5 & $80-M$ & 23.100 & HTS & $143,175.382$ & $583,510.578$ & SURV & ERC-GPS ID\#73 (Nov/Dec 1997) & 111.941 & \\
\hline B8388 & 25.5 & 80-D & 23.100 & HTS & $143,175.382$ & $583,510.578$ & SURV & ERC-GPS ID\#73 (Nov/Dec 1997) & 111.941 & \\
\hline B8393 & 8.5 & 81-S & 25.120 & HTS & $140,494.000$ & $585,375.000$ & EST & ERC-Project planning; L. Peterson (1997) & 108.000 & EST \\
\hline B8392 & 16.5 & 81-M & 25.120 & HTS & $140,494.000$ & $585,375.000$ & EST & ERC-Project planning; L. Peterson (1997) & 108.000 & EST \\
\hline B8391 & 24.5 & 81-D & 25.120 & HTS & $140,494.000$ & $585,375.000$ & EST & ERC-Project planning; L. Peterson (1997) & 108.000 & EST \\
\hline B8396 & 8.5 & $82-\mathrm{S}$ & 25.720 & HTS & $139,595.836$ & $586,252.470$ & SURV & ERC-GPS ID\#75 (Nov/Dec 1997) & 108.814 & \\
\hline B8395 & 14.5 & 82-M & 25.720 & HTS & $139,595.836$ & $586,252.470$ & SURV & ERC-GPS ID\#75 (Nov/Dec 1997) & 108.814 & \\
\hline B8397 & 20.0 & 83-D & 26.230 & HTS & $139,005.466$ & $586,949.357$ & SURV & ERC-GPS ID\#2 (Nov/Dec 1997) & 109.262 & \\
\hline
\end{tabular}


Table 3.1. (contd)

\begin{tabular}{|c|c|c|c|c|c|c|c|c|c|c|}
\hline $\begin{array}{c}\text { Well } \\
\text { Identifier } \\
\text { (HWIS) }\end{array}$ & $\begin{array}{c}\text { Port } \\
\text { Depth } \\
\text { [to top of } \\
\text { opening] } \\
\text { (ft-bgs) }\end{array}$ & $\begin{array}{l}\text { Aquifer Tube } \\
\text { Name } \\
\text { (use in 2009) }\end{array}$ & $\begin{array}{c}\text { HRM } \\
\text { for Site } \\
\text { ("2k") }\end{array}$ & Segment $^{1}$ & $\begin{array}{c}\text { Northing } \\
\text { (m-NAD83) }\end{array}$ & $\begin{array}{c}\text { Easting } \\
\text { (m-NAD83) }\end{array}$ & $\begin{array}{c}\text { Coordinate } \\
\text { Quality }^{2}\end{array}$ & Coordinate Reference & $\begin{array}{c}\text { Ground } \\
\text { Elevation } \\
\text { (m-NAVD88) }\end{array}$ & $\begin{array}{l}\text { Elevation } \\
\text { Quality }^{2}\end{array}$ \\
\hline B8402 & 8.0 & $84-S$ & 26.640 & HTS & $138,683.000$ & $587,477.148$ & SURV & ERC-GPS ID\#5 (Nov/Dec 1997) & 110.895 & \\
\hline B8401 & 14.0 & 84-M & 26.640 & HTS & $138,683.515$ & $587,476.025$ & SURV & ERC-GPS ID\#3 (Nov/Dec 1997) & 110.941 & \\
\hline B8400 & 22.0 & 84-D & 26.640 & HTS & $138,683.945$ & $587,476.487$ & SURV & ERC-GPS ID\#4 (Nov/Dec 1997) & 110.885 & \\
\hline B8405 & 8.0 & $85-S$ & 27.130 & HTS & $138,256.786$ & $588,052.700$ & SURV & ERC-GPS ID\#6 (Nov/Dec 1997) & 111.022 & \\
\hline B8404 & 17.0 & $85-\mathrm{M}$ & 27.130 & HTS & $138,256.786$ & $588,052.700$ & SURV & ERC-GPS ID\#6 (Nov/Dec 1997) & 111.022 & \\
\hline B8403 & 26.0 & 85-D & 27.130 & HTS & $138,256.786$ & $588,052.700$ & SURV & ERC-GPS ID\#6 (Nov/Dec 1997) & 111.022 & \\
\hline B8408 & 7.0 & $86-5$ & 27.390 & HTS & $138,068.820$ & $588,332.623$ & SURV & ERC-GPS ID\#7 (Nov/Dec 1997) & 111.766 & \\
\hline B8407 & 10.0 & $86-\mathrm{M}$ & 27.390 & HTS & $138,068.172$ & $588,333.214$ & SURV & ERC-GPS ID\#8 (Nov/Dec 1997) & 111.767 & \\
\hline B8406 & 26.0 & 86-D & 27.390 & HTS & $138,067.727$ & $588,333.555$ & SURV & ERC-GPS ID\#9 (Nov/Dec 1997) & 111.749 & \\
\hline $\mathrm{C} 6383$ & 7.1 & $\mathrm{C} 6383$ & 28.694 & PO1 & $137,049.790$ & $589,904.200$ & SURV & AST Survey Data 1995-2008-MH-07509.xls & 109.540 & \\
\hline C6384 & 14.3 & C6384 & 28.694 & PO1 & $137,046.730$ & $589,905.770$ & SURV & AST Survey Data 1995-2008-MH-07509.xls & 109.530 & \\
\hline $\mathrm{C} 6353$ & 3.2 & $\mathrm{C} 6353$ & 30.420 & PO1 & $134,707.740$ & $591,487.200$ & SURV & AST Survey Data 1995-2008-MH-07509.xls & 107.540 & \\
\hline C6356 & 3.4 & C6356 & 31.316 & PO1 & $133,699.990$ & $593,014.880$ & SURV & AST Survey Data 1995-2008-MH-07509.xls & 107.960 & \\
\hline C6359 & 4.3 & C6359 & 32.410 & PO1 & $131,807.600$ & $594,209.150$ & SURV & AST Survey Data 1995-2008-MH-07509.xls & 107.490 & \\
\hline C6362 & 6.6 & $\mathrm{C} 6362$ & 33.971 & PO1 & $129,703.410$ & $594,504.590$ & SURV & AST Survey Data 1995-2008-MH-07509.xls & 107.410 & \\
\hline C6365 & 8.7 & C6365 & 34.924 & $\mathrm{PO1}$ & $128,216.740$ & $594,644.690$ & SURV & AST Survey Data 1995-2008-MH-07509.xls & 108.350 & \\
\hline C6368 & 6.4 & C6368 & 36.110 & $\mathrm{PO1}$ & $126,500.860$ & $594,894.340$ & SURV & AST Survey Data 1995-2008-MH-07509.xls & 105.880 & \\
\hline C6371 & 7.6 & C6371 & 36.851 & $\mathrm{PO} 1$ & $125,271.600$ & $594,742.710$ & SURV & AST Survey Data 1995-2008-MH-07509.xls & 106.490 & \\
\hline C6374 & 6.8 & C6374 & 38.274 & PO1 & $123,114.180$ & $594,450.720$ & SURV & AST Survey Data 1995-2008-MH-07509.xls & 106.030 & \\
\hline $\mathrm{C} 6375$ & 8.9 & C6375 & 38.274 & PO1 & $123,115.670$ & $594,448.420$ & SURV & AST Survey Data 1995-2008-MH-07509.xls & 106.150 & \\
\hline C6378 & 5.1 & $\mathrm{C} 6378$ & 39.264 & $\mathrm{PO} 1$ & $121,359.190$ & $594,559.050$ & SURV & AST Survey Data 1995-2008-MH-07509.xls & 105.500 & \\
\hline $\mathrm{C} 6380$ & 1.5 & $\mathrm{C} 6380$ & 40.432 & PO1 & $119,218.610$ & $594,571.830$ & SURV & AST Survey Data 1995-2008-MH-07509.xls & 104.790 & \\
\hline C4347 & 11.6 & AT-3-1-S & 42.080 & $3 F F 5$ & $116,737.054$ & $594,383.725$ & SURV & GPS ID1654 FHI-TimJohnson-March04 & 105.542 & \\
\hline C4346 & 16.8 & AT-3-1-M & 42.080 & 3FF5 & $116,737.016$ & $594,384.907$ & SURV & GPS ID1653 FHI-TimJohnson-March04 & 105.279 & \\
\hline C4348 & 21.0 & AT-3-1-D(2) & 42.080 & 3FF5 & $116,739.488$ & $594,384.954$ & SURV & GPS ID1655 FHI-TimJohnson-March04 & 105.413 & \\
\hline C4345 & 21.1 & AT-3-1-D(1) & 42.080 & 3FF5 & $116,733.965$ & $594,384.577$ & SURV & GPS ID1652 FHI-TimJohnson-March04 & 105.273 & \\
\hline C4350 & 10.8 & AT-3-2-S & 42.235 & 3FF5 & $116,439.560$ & $594,434.714$ & SURV & GPS ID1651 FHI-TimJohnson-March04 & 105.192 & \\
\hline C4349 & 16.6 & AT-3-2-M & 42.235 & $3 F F 5$ & $116,437.665$ & $594,435.306$ & SURV & GPS ID1650 FHI-TimJohnson-March04 & 105.179 & \\
\hline C5277 & 2.7 & 103mArray-US125 & 42.313 & 3FF5 & $116,306.156$ & $594,468.500$ & SURV & PNNL-GPS xyz from Brad Fritz 9/01/06 & 103.930 & \\
\hline C6341 & 11.7 & C6341 & 42.320 & $3 F F 5$ & $116,269.060$ & $594,462.750$ & SURV & AST Survey Data 1995-2008-MH-07509.xls & 105.490 & \\
\hline $\mathrm{C} 6342$ & 17.5 & $\mathrm{C} 6342$ & 42.320 & 3FF5 & $116,293.500$ & $594,463.170$ & SURV & AST Survey Data 1995-2008-MH-07509.xls & 105.650 & \\
\hline $\mathrm{C} 6343$ & 20.6 & C6343 & 42.320 & $3 F F 5$ & $116,295.200$ & $594,463.060$ & SURV & AST Survey Data 1995-2008-MH-07509.xls & 105.450 & \\
\hline C5276 & 6.0 & 103mArray-US100 & 42.332 & $3 F F 5$ & $116,271.547$ & $594,479.000$ & SURV & PNNL-GPS xyz from Brad Fritz 9/01/06 & 104.040 & \\
\hline C5275 & 6.2 & 103mArray-US75 & 42.350 & 3FF5 & $116,237.375$ & $594,490.125$ & SURV & PNNL-GPS xyz from Brad Fritz 9/01/06 & 104.100 & \\
\hline $\mathrm{C} 4642$ & 0.6 & 300 SPR9A-19cm & 42.365 & $3 F F 5$ & $116,209.672$ & $594,495.324$ & SURV & PNNL-GPS xyz from Brad Fritz 9/01/06 & 104.922 & \\
\hline
\end{tabular}


Table 3.1. (contd)

\begin{tabular}{|c|c|c|c|c|c|c|c|c|c|c|}
\hline $\begin{array}{c}\text { Well } \\
\text { Identifier } \\
\text { (HWIS) }\end{array}$ & $\begin{array}{c}\text { Port } \\
\text { Depth } \\
\text { [to top of } \\
\text { opening] } \\
\text { (ft-bgs) }\end{array}$ & $\begin{array}{l}\text { Aquifer Tube } \\
\text { Name } \\
\text { (use in 2009) }\end{array}$ & $\begin{array}{l}\text { HRM } \\
\text { for Site } \\
\text { ("2k") }\end{array}$ & Segment $^{1}$ & $\begin{array}{c}\text { Northing } \\
\text { (m-NAD83) }\end{array}$ & $\begin{array}{c}\text { Easting } \\
\text { (m-NAD83) }\end{array}$ & $\begin{array}{c}\text { Coordinate } \\
\text { Quality }^{2}\end{array}$ & Coordinate Reference & $\begin{array}{c}\text { Ground } \\
\text { Elevation } \\
\text { (m-NAVD88) }\end{array}$ & $\begin{array}{l}\text { Elevation } \\
\text { Quality }^{2}\end{array}$ \\
\hline C4643 & 2.8 & 300 SPR9A-86cm & 42.365 & $3 F F 5$ & $116,209.672$ & $594,495.324$ & SURV & PNNL-GPS xyz from Brad Fritz 9/01/06 & 104.941 & \\
\hline C4644 & 4.7 & 300 SPR9A-142cm & 42.365 & $3 F F 5$ & $116,209.672$ & $594,495.324$ & SURV & PNNL-GPS xyz from Brad Fritz 9/01/06 & 104.946 & \\
\hline C5274 & 6.1 & 103mArray-US50 & 42.367 & $3 F F 5$ & $116,207.422$ & $594,500.000$ & SURV & PNNL-GPS xyz from Brad Fritz 9/01/06 & 104.050 & \\
\hline C5273 & 5.6 & 103mArray-US25 & 42.377 & $3 F F 5$ & $116,188.852$ & $594,505.563$ & SURV & PNNL-GPS xyz from Brad Fritz 9/01/06 & 103.900 & \\
\hline (none) & 3.9 & $300-3-3 B-120 \mathrm{~cm}$ & 42.389 & $3 F F 5$ & $116,166.500$ & $594,516.000$ & SURV & PNNL-GPS xyz from Brad Fritz 9/01/06 & 104.480 & EST \\
\hline C4646 & 12.3 & 300-3-3B-376cm & 42.389 & 3FF5 & $116,165.529$ & $594,516.017$ & SURV & PNNL-GPS xyz from Brad Fritz 9/01/06 & 104.480 & \\
\hline C4740 & 17.0 & 300-3-3B-518cm & 42.389 & $3 F F 5$ & $116,165.530$ & $594,516.020$ & EST & PNNL-GPS xyz from Brad Fritz 9/01/06 & 103.680 & \\
\hline C4741 & 13.4 & $300-3-3 c-409 \mathrm{~cm}$ & 42.390 & $3 F F 5$ & $116,167.350$ & $594,524.410$ & EST & PNNL-GPS xyz from Brad Fritz 9/01/06 & 103.290 & \\
\hline C4742 & 19.3 & $300-3-3 C-589 \mathrm{~cm}$ & 42.390 & $3 F F 5$ & $116,167.650$ & $594,524.710$ & EST & PNNL-GPS xyz from Brad Fritz 9/01/06 & 103.290 & \\
\hline (none) & 2.6 & $300-3-3-80 \mathrm{~cm}$ & 42.391 & $3 F F 5$ & $116,163.600$ & $594,507.000$ & SURV & PNNL-GPS xyz from Brad Fritz 9/01/06 & 105.000 & EST \\
\hline C4690 & 4.1 & 300-3-3A-124cm & 42.391 & $3 F F 5$ & $116,163.246$ & $594,512.706$ & EST & PNNL-GPS xyz from Brad Fritz 9/01/06 & 104.250 & \\
\hline C4645 & 13.5 & $300-3-3 A-410 \mathrm{~cm}$ & 42.391 & $3 F F 5$ & $116,163.246$ & $594,512.206$ & SURV & PNNL-GPS xyz from Brad Fritz 9/01/06 & 105.050 & \\
\hline C4739 & 19.0 & $300-3-3 A-579 \mathrm{~cm}$ & 42.391 & $3 F F 5$ & $116,163.250$ & $594,512.210$ & EST & PNNL-GPS xyz from Brad Fritz 9/01/06 & 104.250 & \\
\hline C5272 & 6.9 & 103mArray-AT3A & 42.391 & $3 F F 5$ & $116,163.086$ & $594,512.438$ & SURV & PNNL-GPS xyz from Brad Fritz 9/01/06 & 104.300 & \\
\hline C4353 & 6.9 & AT-3-3-S & 42.392 & 3FF5 & $116,163.568$ & $594,505.024$ & SURV & PNNL-GPS xyz from Brad Fritz 9/01/06 & 105.003 & \\
\hline C4352 & 15.1 & AT-3-3-M & 42.392 & 3FF5 & $116,160.406$ & $594,505.464$ & SURV & PNNL-GPS xyz from Brad Fritz 9/01/06 & 105.119 & \\
\hline C4351 & 29.1 & AT-3-3-D & 42.392 & $3 F F 5$ & $116,159.062$ & $594,505.631$ & SURV & PNNL-GPS xyz from Brad Fritz 9/01/06 & 104.956 & \\
\hline C5278 & 5.6 & 103mArray-DS25 & 42.404 & $3 F F 5$ & $116,137.734$ & $594,518.125$ & SURV & PNNL-GPS xyz from Brad Fritz 9/01/06 & 103.900 & \\
\hline C5279 & 5.2 & 103mArray-DS50 & 42.412 & 3FF5 & $116,123.367$ & $594,521.250$ & SURV & PNNL-GPS xyz from Brad Fritz 9/01/06 & 103.800 & \\
\hline C6344 & 7.3 & C6344 & 42.431 & 3FF5 & $116,077.560$ & $594,525.240$ & SURV & AST Survey Data 1995-2008-MH-07509.xls & 105.350 & \\
\hline C5281 & 3.2 & DS75-100cm & 42.434 & 3FF5 & $116,082.367$ & $594,530.125$ & SURV & PNNL-GPS xyz from Brad Fritz 9/01/06 & 104.600 & \\
\hline C5280 & 7.9 & 103mArray-DS75 & 42.434 & $3 F F 5$ & $116,082.367$ & $594,530.125$ & SURV & PNNL-GPS xyz from Brad Fritz 9/01/06 & 104.600 & \\
\hline C5282 & 10.5 & DS75-319cm & 42.434 & $3 F F 5$ & $116,082.367$ & $594,530.125$ & SURV & PNNL-GPS xyz from Brad Fritz 9/01/06 & 104.600 & \\
\hline C4356 & 7.0 & AT-3-4-S & 42.492 & 3FF5 & $115,978.828$ & $594,542.624$ & SURV & GPS ID1645 FHI-TimJohnson-March04 & 105.335 & \\
\hline C4355 & 9.2 & AT-3-4-M & 42.492 & 3FF5 & $115,976.383$ & $594,543.643$ & SURV & GPS ID1644 FHI-TimJohnson-March04 & 105.296 & \\
\hline C4354 & 12.0 & AT-3-4-D & 42.492 & 3FF5 & $115,980.412$ & $594,541.904$ & SURV & GPS ID1646 FHI-TimJohnson-March04 & 105.299 & \\
\hline C6347 & 9.8 & C6347 & 42.549 & 3FF5 & $115,859.250$ & $594,578.360$ & SURV & AST Survey Data 1995-2008-MH-07509.xls & 105.070 & \\
\hline C6348 & 12.1 & C6348 & 42.549 & 3FF5 & $115,861.980$ & $594,577.930$ & SURV & AST Survey Data 1995-2008-MH-07509.xls & 105.140 & \\
\hline C4358 & 7.7 & AT-3-5-S & 42.608 & 3FF5 & $115,749.968$ & $594,602.159$ & SURV & GPS ID1642 FHI-TimJohnson-March04 & 105.104 & \\
\hline C4357 & 12.2 & AT-3-5-M & 42.608 & 3FF5 & $115,753.181$ & $594,600.678$ & SURV & GPS ID1643 FHI-TimJohnson-March04 & 105.198 & \\
\hline C6350 & 8.4 & C6350 & 42.668 & 3FF5 & $115,642.050$ & $594,638.090$ & SURV & AST Survey Data 1995-2008-MH-07509.xls & 105.200 & \\
\hline C6351 & 14.2 & C6351 & 42.668 & 3FF5 & $115,640.530$ & $594,638.560$ & SURV & AST Survey Data 1995-2008-MH-07509.xls & 105.360 & \\
\hline C4361 & 9.6 & AT-3-6-S & 42.720 & 3FF5 & $115,548.309$ & $594,663.336$ & SURV & GPS ID1641 FHI-TimJohnson-March04 & 104.892 & \\
\hline C4360 & 21.8 & AT-3-6-M & 42.720 & 3FF5 & $115,545.896$ & $594,663.856$ & SURV & GPS ID1640 FHI-TimJohnson-March04 & 104.944 & \\
\hline C4359 & 38.6 & AT-3-6-D & 42.720 & 3FF5 & $115,547.715$ & $594,663.947$ & SURV & GPS ID1639 FHI-TimJohnson-March04 & 104.837 & \\
\hline
\end{tabular}


Table 3.1. (contd)

\begin{tabular}{|c|c|c|c|c|c|c|c|c|c|c|}
\hline $\begin{array}{c}\text { Well } \\
\text { Identifier } \\
\text { (HWIS) }\end{array}$ & $\begin{array}{c}\text { Port } \\
\text { Depth } \\
\text { [to top of } \\
\text { opening] } \\
\text { (ft-bgs) }\end{array}$ & $\begin{array}{c}\text { Aquifer Tube } \\
\text { Name } \\
\text { (use in 2009) }\end{array}$ & $\begin{array}{l}\text { HRM } \\
\text { for Site } \\
\text { ("2k") }\end{array}$ & Segment $^{1}$ & $\begin{array}{c}\text { Northing } \\
\text { (m-NAD83) }\end{array}$ & $\begin{array}{c}\text { Easting } \\
\text { (m-NAD83) }\end{array}$ & $\begin{array}{c}\text { Coordinate } \\
\text { Quality }^{2}\end{array}$ & Coordinate Reference & $\begin{array}{c}\begin{array}{c}\text { Ground } \\
\text { Elevation } \\
\text { (m-NAVD88) }\end{array} \\
\end{array}$ & $\begin{array}{l}\text { Elevation } \\
\text { Quality }^{2}\end{array}$ \\
\hline C4364 & 8.6 & AT-3-7-S & 42.820 & 3FF5 & $115,352.844$ & $594,705.293$ & SURV & GPS ID1637 FHI-TimJohnson-March04 & 104.887 & \\
\hline C4363 & 20.8 & AT-3-7-M & 42.820 & 3FF5 & $115,350.598$ & $594,706.026$ & SURV & GPS ID1638 FHI-TimJohnson-March04 & 104.930 & \\
\hline C4362 & 37.3 & AT-3-7-D & 42.820 & 3FF5 & $115,348.620$ & $594,706.323$ & SURV & GPS ID1636 FHI-TimJohnson-March04 & 104.870 & \\
\hline C4367 & 8.0 & AT-3-8-S & 42.940 & 3FF5 & $115,127.249$ & $594,761.597$ & SURV & GPS ID1633 FHI-TimJohnson-March04 & 104.990 & \\
\hline C4366 & 14.0 & AT-3-8-M & 42.940 & $3 F F 5$ & $115,129.001$ & $594,760.643$ & SURV & GPS ID1634 FHI-TimJohnson-March04 & 104.940 & \\
\hline C4365 & 20.1 & AT-3-8-D & 42.940 & $3 F F 5$ & $115,130.709$ & $594,759.951$ & SURV & GPS ID1635 FHI-TimJohnson-March04 & 105.049 & \\
\hline
\end{tabular}


Table 3.2. Historical Names Used for Some Aquifer Sampling Tube Sites

\begin{tabular}{|c|c|c|c|c|c|c|c|}
\hline $\begin{array}{c}\text { Well } \\
\text { Identifier } \\
\text { (HWIS) }\end{array}$ & $\begin{array}{c}\text { Port } \\
\text { Depth } \\
\text { [to top of } \\
\text { opening] } \\
\text { (ft-bgs) }\end{array}$ & $\begin{array}{c}\text { Aquifer Tube } \\
\text { Name } \\
\text { (use in 2009) }\end{array}$ & $\begin{array}{l}\text { HRM } \\
\text { for Site } \\
\text { ("2k") }\end{array}$ & Segment $^{1}$ & $\begin{array}{l}\text { Name as Used } \\
\text { in Reference } \\
\text { (historical use) }\end{array}$ & Name Reference & $\begin{array}{l}\text { Year of } \\
\text { Name } \\
\text { Use }^{2}\end{array}$ \\
\hline C4376 & 8.0 & AT-B-1-S & 3.570 & BC5 & 166-B-9 & HEIS2_ADM_WELL table & 2001 \\
\hline C4375 & 13.3 & AT-B-1-M & 3.570 & BC5 & 166-B-8 & HEIS2_ADM_WELL table & 2001 \\
\hline C4378 & 8.6 & AT-B-2-S & 3.660 & BC5 & 166-B-11 & HEIS2_ADM_WELL table & 2001 \\
\hline C4379 & 14.0 & AT-B-2-M & 3.660 & BC5 & 166-B-12 & HEIS2_ADM_WELL table & 2001 \\
\hline C4377 & 19.0 & AT-B-2-D & 3.660 & BC5 & 166-B-10 & HEIS2_ADM_WELL table & 2001 \\
\hline C4382 & 8.1 & AT-B-3-S & 4.020 & BC5 & 166-B-15 & HEIS2_ADM_WELL table & 2001 \\
\hline C4381 & 14.2 & AT-B-3-M & 4.020 & BC5 & 166-B-14 & HEIS2_ADM_WELL table & 2001 \\
\hline C4380 & 23.2 & AT-B-3-D & 4.020 & BC5 & 166-B-13 & HEIS2_ADM_WELL table & 2001 \\
\hline C4368 & 7.5 & AT-B-4-S & 4.440 & BC5 & 166-B-1 & HEIS2_ADM_WELL table & 2001 \\
\hline C4371 & 6.8 & AT-B-7-S & 4.620 & BC5 & 166-B-4 & HEIS2_ADM_WELL table & 2001 \\
\hline C4370 & 13.3 & AT-B-7-M & 4.620 & BC5 & 166-B-3 & HEIS2_ADM_WELL table & 2001 \\
\hline C4369 & 18.1 & AT-B-7-D & 4.620 & BC5 & 166-B-2 & HEIS2_ADM_WELL table & 2001 \\
\hline C4374 & 9.6 & AT-B-5-S & 4.770 & BC5 & 166-B-7 & HEIS2_ADM_WELL table & 2001 \\
\hline $\mathrm{C} 4373$ & 16.2 & AT-B-5-M & 4.770 & BC5 & 166-B-6 & HEIS2_ADM_WELL table & 2001 \\
\hline C4372 & 24.0 & AT-B-5-D & 4.770 & BC5 & 166-B-5 & HEIS2_ADM_WELL table & 2001 \\
\hline $\mathrm{C} 4341$ & 9.2 & AT-K-1-S & 6.270 & KR4 & $166-\mathrm{K}-16$ & HEIS2_ADM_WELL table & 2001 \\
\hline C4340 & 15.0 & AT-K-1-M & 6.270 & KR4 & $166-\mathrm{K}-15$ & HEIS2_ADM_WELL table & 2001 \\
\hline C4339 & 21.7 & AT-K-1-D & 6.270 & KR4 & $166-\mathrm{K}-14$ & HEIS2_ADM_WELL table & 2001 \\
\hline C4329 & 15.0 & AT-K-2-S & 6.640 & KR4 & $166-K-3$ & HEIS2_ADM_WELL table & 2001 \\
\hline $\mathrm{C} 4327$ & 18.2 & AT-K-2-M & 6.640 & KR4 & 166-K-1 & HEIS2_ADM_WELL table & 2001 \\
\hline C4328 & 22.3 & AT-K-2-D & 6.640 & KR4 & $166-\mathrm{K}-2$ & HEIS2_ADM_WELL table & 2001 \\
\hline C4344 & 13.4 & AT-K-3-S & 7.000 & KR4 & $166-K-19$ & HEIS2_ADM_WELL table & 2001 \\
\hline $\mathrm{C} 4343$ & 17.8 & AT-K-3-M & 7.000 & KR4 & $166-\mathrm{K}-18$ & HEIS2_ADM_WELL table & 2001 \\
\hline $\mathrm{C} 4342$ & 23.0 & AT-K-3-D & 7.000 & KR4 & $166-\mathrm{K}-17$ & HEIS2_ADM_WELL table & 2001 \\
\hline C4338 & 11.0 & AT-K-4-S & 7.880 & KR4 & $166-K-13$ & HEIS2_ADM_WELL table & 2001 \\
\hline $\mathrm{C} 4337$ & 13.2 & AT-K-4-M & 7.880 & KR4 & $166-\mathrm{K}-12$ & HEIS2_ADM_WELL table & 2001 \\
\hline C4336 & 15.3 & AT-K-4-D & 7.880 & KR4 & $166-K-11$ & HEIS2_ADM_WELL table & 2001 \\
\hline C4335 & 10.5 & AT-K-5-S & 8.090 & KR4 & $166-\mathrm{K}-10$ & HEIS2_ADM_WELL table & 2001 \\
\hline C4331 & 15.7 & AT-K-5-M & 8.090 & KR4 & $166-K-5$ & HEIS2_ADM_WELL table & 2001 \\
\hline $\mathrm{C} 4330$ & 21.1 & AT-K-5-D & 8.090 & KR4 & $166-K-4$ & HEIS2_ADM_WELL table & 2001 \\
\hline C4333 & 11.4 & AT-K-6-S & 8.310 & KR4 & $166-K-8$ & HEIS2_ADM_WELL table & 2001 \\
\hline $\mathrm{C} 4334$ & 15.3 & AT-K-6-M & 8.310 & KR4 & 166-K-9 & HEIS2_ADM_WELL table & 2001 \\
\hline $\mathrm{C} 4332$ & 21.6 & AT-K-6-D & 8.310 & KR4 & $166-\mathrm{K}-7$ & HEIS2_ADM_WELL table & 2001 \\
\hline \multirow[t]{4}{*}{ C3383 } & 3.0 & Redox-4-3.0 & 10.125 & HR3D & Redox-4-3.0 & FY2004 refurbishment (PNNL) & 2004 \\
\hline & & & & & 166-D-4 & HEIS2_ADM_WELL table & 2001 \\
\hline & & & & & ISRM-04 & DOE/RL-2000-59 & 2000 \\
\hline & & & & & REDOX0403.0 & PNNL-13349 & 2000 \\
\hline \multirow[t]{4}{*}{ C3515 } & 6.0 & Redox-4-6.0 & 10.125 & HR3D & Redox-4-6.0 & FY2004 refurbishment (PNNL) & 2004 \\
\hline & & & & & 166-D-4B & HEIS2_ADM_WELL table & 2001 \\
\hline & & & & & ISRM-04 & DOE/RL-2000-59 & 2000 \\
\hline & & & & & REDOX0406.0 & PNNL-13349 & 2000 \\
\hline \multirow[t]{8}{*}{ C3384 } & 3.3 & Redox-3-3.3 & 10.180 & HR3D & Redox-3-3.3 & FY2004 refurbishment (PNNL) & 2004 \\
\hline & & & & & $166-D-3$ & PNNL-14444 & 2003 \\
\hline & & & & & Redox-03 & WMP-18051 & 2003 \\
\hline & & & & & Redox-03 & BHI-01624 & 2002 \\
\hline & & & & & REDOX-3-03 & BHI-01494 & 2001 \\
\hline & & & & & $166-D-3$ & HEIS2_ADM_WELL table & 2001 \\
\hline & & & & & ISRM-03 & DOE/RL-2000-59 & 2000 \\
\hline & & & & & REDOX0303.3 & PNNL-13349 & 2000 \\
\hline
\end{tabular}


Table 3.2. (contd)

\begin{tabular}{|c|c|c|c|c|c|c|c|}
\hline $\begin{array}{c}\text { Well } \\
\text { Identifier } \\
\text { (HWIS) }\end{array}$ & $\begin{array}{c}\text { Port } \\
\text { Depth } \\
\text { [to top of } \\
\text { opening] } \\
\text { (ft-bgs) }\end{array}$ & $\begin{array}{c}\text { Aquifer Tube } \\
\text { Name } \\
\text { (use in 2009) }\end{array}$ & $\begin{array}{l}\text { HRM } \\
\text { for Site } \\
\text { ("2k") }\end{array}$ & Segment $^{1}$ & $\begin{array}{l}\text { Name as Used } \\
\text { in Reference } \\
\text { (historical use) }\end{array}$ & Name Reference & $\begin{array}{c}\text { Year of } \\
\text { Name } \\
\text { Use }^{2}\end{array}$ \\
\hline \multirow[t]{7}{*}{ C3514 } & 4.6 & Redox-3-4.6 & 10.180 & HR3D & Redox-3-4.6 & FY2004 refurbishment (PNNL) & 2004 \\
\hline & & & & & 166-D-3B & PNNL-14444 & 2003 \\
\hline & & & & & Redox-03 & WMP-18051 & 2003 \\
\hline & & & & & Redox-03 & BHI-01624 & 2002 \\
\hline & & & & & 166-D-3B & HEIS2_ADM_WELL table & 2001 \\
\hline & & & & & ISRM-03 & DOE/RL-2000-59 & 2000 \\
\hline & & & & & REDOX0304.6 & PNNL-13349 & 2000 \\
\hline \multirow[t]{7}{*}{ C3385 } & 3.0 & Redox-2-3.0 & 10.240 & HR3D & Redox-2-3.0 & FY2004 refurbishment (PNNL) & 2004 \\
\hline & & & & & 166-D-2 & PNNL-14444 & 2003 \\
\hline & & & & & Redox-02 & WMP-18051 & 2003 \\
\hline & & & & & Redox-02 & BHI-01624 & 2002 \\
\hline & & & & & 166-D-2 & HEIS2_ADM_WELL table & 2001 \\
\hline & & & & & ISRM-02 & DOE/RL-2000-59 & 2000 \\
\hline & & & & & REDOX0203.0 & PNNL-13349 & 2000 \\
\hline \multirow[t]{8}{*}{ C3513 } & 6.0 & Redox-2-6.0 & 10.240 & HR3D & Redox-2-6.0 & FY2004 refurbishment (PNNL) & 2004 \\
\hline & & & & & 166-D-2B & PNNL-14444 & 2003 \\
\hline & & & & & Redox-02 & WMP-18051 & 2003 \\
\hline & & & & & Redox-02 & BHI-01624 & 2002 \\
\hline & & & & & REDOX-2-06 & BHI-01494 & 2001 \\
\hline & & & & & 166-D-2B & HEIS_ADM_WELL table & 2001 \\
\hline & & & & & ISRM-02 & DOE/RL-2000-59 & 2000 \\
\hline & & & & & REDOX0206.0 & PNNL-13349 & 2000 \\
\hline \multirow[t]{4}{*}{ C3382 } & 3.3 & Redox-1-3.3 & 10.295 & HR3D & Redox-1-3.3 & FY2004 refurbishment (PNNL) & 2004 \\
\hline & & & & & 166-D-1 & HEIS2_ADM_WELL table & 2001 \\
\hline & & & & & ISRM-01 & DOE/RL-2000-59 & 2000 \\
\hline & & & & & REDOX0103.3 & PNNL-13349 & 2000 \\
\hline \multirow[t]{5}{*}{ C3512 } & 6.0 & Redox-1-6.0 & 10.295 & HR3D & Redox-1-6.0 & FY2004 refurbishment (PNNL) & 2004 \\
\hline & & & & & REDOX-1-06 & BHI-01494 & 2001 \\
\hline & & & & & 166-D-1B & HEIS2_ADM_WELL table & 2001 \\
\hline & & & & & ISRM-01 & DOE/RL-2000-59 & 2000 \\
\hline & & & & & REDOX0106.0 & PNNL-13349 & 2000 \\
\hline C4307 & 7.0 & AT-D-1-S & 10.420 & HR3D & 166-D-7 & HEIS2_ADM_WELL table & 2001 \\
\hline C4306 & 10.8 & AT-D-1-M & 10.420 & HR3D & 166-D-6 & HEIS2_ADM_WELL table & 2001 \\
\hline C4305 & 13.3 & AT-D-1-D & 10.420 & HR3D & 166-D-5 & HEIS2_ADM_WELL table & 2001 \\
\hline $\mathrm{C} 4314$ & 12.4 & AT-D-4-S & 10.580 & HR3D & 166-D-14 & HEIS2_ADM_WELL table & 2001 \\
\hline C4315 & 13.8 & AT-D-4-M & 10.580 & HR3D & 166-D-15 & HEIS2_ADM_WELL table & 2001 \\
\hline $\mathrm{C} 4316$ & 15.7 & AT-D-4-D & 10.580 & HR3D & 166-D-16 & HEIS2_ADM_WELL table & 2001 \\
\hline $\mathrm{C} 4310$ & 14.3 & AT-D-2-S & 10.660 & HR3D & 166-D-10 & HEIS2_ADM_WELL table & 2001 \\
\hline C4309 & 16.3 & AT-D-2-M & 10.660 & HR3D & 166-D-9 & HEIS2_ADM_WELL table & 2001 \\
\hline C4308 & 26.5 & AT-D-2-D & 10.660 & HR3D & 166-D-8 & HEIS2_ADM_WELL table & 2001 \\
\hline C4313 & 7.3 & AT-D-3-S & 10.750 & HR3D & 166-D-13 & HEIS2_ADM_WELL table & 2001 \\
\hline $\mathrm{C} 4312$ & 8.8 & AT-D-3-M & 10.750 & HR3D & $166-\mathrm{D}-12$ & HEIS2_ADM_WELL table & 2001 \\
\hline $\mathrm{C} 4311$ & 11.8 & AT-D-3-D & 10.750 & HR3D & 166-D-11 & HEIS2_ADM_WELL table & 2001 \\
\hline C4318 & 6.8 & AT-D-5-M & 11.310 & HR3D & 166-D-18 & HEIS2_ADM_WELL table & 2001 \\
\hline $\mathrm{C} 4317$ & 8.8 & AT-D-5-D & 11.310 & HR3D & 166-D-17 & HEIS2_ADM_WELL table & 2001 \\
\hline C4321 & 6.2 & AT-H-1-S & 15.160 & $\mathrm{HR} 3 \mathrm{H}$ & $166-\mathrm{H}-3$ & HEIS2_ADM_WELL table & 2001 \\
\hline C4320 & 11.0 & AT-H-1-M & 15.160 & $\mathrm{HR} 3 \mathrm{H}$ & $166-\mathrm{H}-2$ & HEIS2_ADM_WELL table & 2001 \\
\hline C4319 & 12.8 & AT-H-1-D & 15.160 & $\mathrm{HR} 3 \mathrm{H}$ & $166-\mathrm{H}-1$ & HEIS2_ADM_WELL table & 2001 \\
\hline C4324 & 5.3 & AT-H-2-S & 15.200 & $\mathrm{HR} 3 \mathrm{H}$ & $166-\mathrm{H}-6$ & HEIS2_ADM_WELL table & 2001 \\
\hline $\mathrm{C} 4323$ & 9.2 & AT-H-2-M & 15.200 & $\mathrm{HR} 3 \mathrm{H}$ & $166-\mathrm{H}-5$ & HEIS2_ADM_WELL table & 2001 \\
\hline
\end{tabular}


Table 3.2. (contd)

\begin{tabular}{|c|c|c|c|c|c|c|c|}
\hline $\begin{array}{c}\text { Well } \\
\text { Identifier } \\
\text { (HWIS) }\end{array}$ & $\begin{array}{c}\text { Port } \\
\text { Depth } \\
\text { [to top of } \\
\text { opening] } \\
\text { (ft-bgs) }\end{array}$ & $\begin{array}{c}\text { Aquifer Tube } \\
\text { Name } \\
\text { (use in 2009) }\end{array}$ & $\begin{array}{l}\text { HRM } \\
\text { for Site } \\
\text { ("2k") }\end{array}$ & Segment $^{1}$ & $\begin{array}{l}\text { Name as Used } \\
\text { in Reference } \\
\text { (historical use) }\end{array}$ & Name Reference & $\begin{array}{l}\text { Year of } \\
\text { Name } \\
\text { Use }^{2}\end{array}$ \\
\hline C4322 & 12.0 & AT-H-2-D & 15.200 & $\mathrm{HR} 3 \mathrm{H}$ & $166-\mathrm{H}-4$ & HEIS2_ADM_WELL table & 2001 \\
\hline C4326 & 5.3 & AT-H-3-S & 15.230 & HR3H & $166-\mathrm{H}-8$ & HEIS2_ADM_WELL table & 2001 \\
\hline C4325 & 7.3 & AT-H-3-D & 15.230 & $\mathrm{HR} 3 \mathrm{H}$ & $166-\mathrm{H}-7$ & HEIS2_ADM_WELL table & 2001 \\
\hline C4391 & 10.3 & AT-F-1-S & 19.220 & FR3 & $166-\mathrm{F}-9$ & HEIS2_ADM_WELL table & 2001 \\
\hline C4390 & 18.1 & AT-F-1-M & 19.220 & FR3 & $166-\mathrm{F}-8$ & HEIS2_ADM_WELL table & 2001 \\
\hline C4389 & 26.1 & AT-F-1-D & 19.220 & FR3 & $166-\mathrm{F}-7$ & HEIS2_ADM_WELL table & 2001 \\
\hline C4394 & 7.5 & AT-F-2-S & 20.110 & FR3 & $166-\mathrm{F}-12$ & HEIS2_ADM_WELL table & 2001 \\
\hline C4393 & 13.6 & AT-F-2-M & 20.110 & FR3 & $166-F-11$ & HEIS2_ADM_WELL table & 2001 \\
\hline C4392 & 19.3 & AT-F-2-D & 20.110 & FR3 & $166-F-10$ & HEIS2_ADM_WELL table & 2001 \\
\hline C4385 & 6.0 & AT-F-3-S & 20.420 & FR3 & $166-\mathrm{F}-3$ & HEIS2_ADM_WELL table & 2001 \\
\hline C4384 & 10.8 & AT-F-3-M & 20.420 & FR3 & $166-\mathrm{F}-2$ & HEIS2_ADM_WELL table & 2001 \\
\hline C4383 & 14.1 & AT-F-3-D & 20.420 & FR3 & $166-\mathrm{F}-1$ & HEIS2_ADM_WELL table & 2001 \\
\hline C4388 & 7.2 & AT-F-4-S & 21.000 & FR3 & $166-\mathrm{F}-6$ & HEIS2_ADM_WELL table & 2001 \\
\hline C4387 & 18.0 & AT-F-4-M & 21.000 & FR3 & $166-\mathrm{F}-5$ & HEIS2_ADM_WELL table & 2001 \\
\hline C4386 & 32.3 & AT-F-4-D & 21.000 & FR3 & $166-\mathrm{F}-4$ & HEIS2_ADM_WELL table & 2001 \\
\hline C4347 & 11.6 & AT-3-1-S & 42.080 & $3 F F 5$ & $366-1-3$ & HEIS2_ADM_WELL table & 2001 \\
\hline C4346 & 16.8 & AT-3-1-M & 42.080 & 3FF5 & $366-1-2$ & HEIS2_ADM_WELL table & 2001 \\
\hline C4348 & 21.0 & AT-3-1-D(2) & 42.080 & 3FF5 & $366-1-4$ & HEIS2_ADM_WELL table & 2001 \\
\hline C4345 & 21.1 & AT-3-1-D(1) & 42.080 & 3FF5 & $366-1-1$ & HEIS2_ADM_WELL table & 2001 \\
\hline C4350 & 10.8 & AT-3-2-S & 42.235 & 3FF5 & $366-1-6$ & HEIS2_ADM_WELL table & 2001 \\
\hline C4349 & 16.6 & AT-3-2-M & 42.235 & 3FF5 & $366-1-5$ & HEIS2_ADM_WELL table & 2001 \\
\hline C4353 & 6.9 & AT-3-3-S & 42.392 & 3FF5 & $366-2-3$ & HEIS2_ADM_WELL table & 2001 \\
\hline C4352 & 15.1 & AT-3-3-M & 42.392 & 3FF5 & $366-2-2$ & HEIS2_ADM_WELL table & 2001 \\
\hline C4351 & 29.1 & AT-3-3-D & 42.392 & 3FF5 & $366-2-1$ & HEIS2_ADM_WELL table & 2001 \\
\hline C4356 & 7.0 & AT-3-4-S & 42.492 & 3FF5 & $366-3-3$ & HEIS2_ADM_WELL table & 2001 \\
\hline C4355 & 9.2 & AT-3-4-M & 42.492 & 3FF5 & $366-3-2$ & HEIS2_ADM_WELL table & 2001 \\
\hline C4354 & 12.0 & AT-3-4-D & 42.492 & 3FF5 & $366-3-1$ & HEIS2_ADM_WELL table & 2001 \\
\hline C4358 & 7.7 & AT-3-5-S & 42.608 & 3FF5 & $366-4-2$ & HEIS2_ADM_WELL table & 2001 \\
\hline C4357 & 12.2 & AT-3-5-M & 42.608 & 3FF5 & $366-4-1$ & HEIS2_ADM_WELL table & 2001 \\
\hline C4361 & 9.6 & AT-3-6-S & 42.720 & $3 F F 5$ & $366-4-5$ & HEIS2_ADM_WELL table & 2001 \\
\hline C4360 & 21.8 & AT-3-6-M & 42.720 & 3FF5 & $366-4-4$ & HEIS2_ADM_WELL table & 2001 \\
\hline C4359 & 38.6 & AT-3-6-D & 42.720 & 3FF5 & $366-4-3$ & HEIS2_ADM_WELL table & 2001 \\
\hline C4364 & 8.6 & AT-3-7-S & 42.820 & 3FF5 & $366-4-8$ & HEIS2_ADM_WELL table & 2001 \\
\hline C4363 & 20.8 & AT-3-7-M & 42.820 & 3FF5 & $366-4-7$ & HEIS2_ADM_WELL table & 2001 \\
\hline C4362 & 37.3 & AT-3-7-D & 42.820 & 3FF5 & $366-4-6$ & HEIS2_ADM_WELL table & 2001 \\
\hline C4367 & 8.0 & AT-3-8-S & 42.940 & 3FF5 & $366-4-11$ & HEIS2_ADM_WELL table & 2001 \\
\hline C4366 & 14.0 & AT-3-8-M & 42.940 & 3FF5 & $366-4-10$ & HEIS2_ADM_WELL table & 2001 \\
\hline C4365 & 20.1 & AT-3-8-D & 42.940 & 3FF5 & $366-4-9$ & HEIS2_ADM_WELL table & 2001 \\
\hline
\end{tabular}




\subsection{Riverbank Springs}

Riverbank springs are observed at many locations along the Hanford Reach shoreline during periods of low river stage. As a general rule, most riverbank springs become visible when the Columbia River flow drops below 75,000 cubic feet per second (cfs). During high river stage, river water moves into the riverbank and interacts with groundwater that approaches the channel. When the river level drops, this bank storage water drains back toward the channel, often becoming exposed at the surface as springs or seepage areas along the beach. Figure 4.1 is a photo of a riverbank spring emerging at the 100-N Area shoreline. The composition of spring water may vary from nearly pure river water when the spring first emerges, to becoming more dominated by groundwater as the low river stage continues.

\subsection{Riverbank Springs Naming Conventions}

The naming system for the 1982-1983 springs investigation (McCormack and Carlile 1984) used HRM signposts, which subdivide the shoreline between Vernita Bridge and North Richland into 46 segments, each approximately one-mile long. A spring site name was composed from the nearest upstream signpost, to which a suffix was added that indicated the sequence of the site downstream of the signpost. For example, "3-5” indicated the fifth spring observed downstream from HRM \#3. During a second comprehensive investigation of riverbank springs in 1988 (Dirkes 1990), the location names used followed the convention established by the earlier 1982 investigation. However, the analytical results are tabulated according to HRM position and are not given discreet location names (Dirkes 1990).

As part of a third comprehensive investigation of riverbank springs conducted in fall 1991, PNNL staff outlined all springs areas previously observed and sampled on the newly-acquired 1:2,000 series of topographic maps for the Hanford Site. A new naming system for spring locations was devised, again based on the HRM signposts, and efforts were made to associate all previously sampled locations with the new naming convention (DOE-RL 1992). The new name format consisted of the location to a tenth of a signpost position, followed by a suffix used to indicate multiple springs at the same approximate location. For example, springs 057-1, 057-2, etc. would indicate multiple springs at HRM 5.7, a location seven tenths of the distance downstream from HRM \#5 to HRM \#6.

Following the 1991 investigation, the method for naming riverbank springs continued to follow essentially the same scheme, but with some enhancements. A prefix (S) was added to identify the location as a spring and was combined with a letter that identifies the nearest reactor area. A spring site in the 100-K Area would thus be prefaced with "SK-" under this scheme, e.g., "SK-057-1."

This report proposes establishing a unique identifier for each riverbank spring site that is routinely monitored or is persistent. The five-digit alpha-numeric identifier would be analogous to the well identifiers assigned in HWIS to groundwater monitoring wells and aquifer tubes. The new identifier could be used in electronic databases to provide a unique tag for each location sampled. (Note: The proposed spring location identifiers listed in Tables 4.1 and 4.2 that follow have not been formally implemented as of the date of publication for this report).

Also, a new, simplified convention for assigning description names to riverbank springs is proposed in this report (see Chapter 8). Because acceptance of a naming convention depends on the needs of numerous users, and also on the need to maintain some continuity with names used previously, the 
proposed convention has not yet been adopted in the sampling and analysis schedule databases for groundwater (GW) and surface water (SW) sample media. Based on acceptance from the user community at the Hanford Site, a future revision of this report will reflect any new conventions that may be adopted.

\subsection{Riverbank Spring Names and Coordinates}

The list of sites where riverbank springs have been observed and possibly sampled during past investigations is presented in Table 4.1. Each site listed is believed to represent a discreet occurrence of a spring. The inventory is the result of a detailed review of the location descriptions presented in the earlier reports discussed in Chapter 2. In many instances, a single location has been previously described using a variety of descriptive names. The review included interviews with investigators who have had abundant field experience along the shoreline, and also by referencing back to field notebooks kept during sampling events. The columns in Table 4.1 are defined as follows:

- Site Identifier (proposed) - Identifier code proposed for each riverbank spring site-for internal data management purposes. This code would be used in the same way as the well identifier code assigned in the HWIS for monitoring wells, boreholes, and aquifer sampling tubes.

- Spring Name (in use 2009) - The name in use during 2009 for a site that is sampled routinely under various monitoring programs. For those sites not currently sampled, the most commonly appearing name in historical reports is listed. In some instances, a riverbank spring had been identified on a map, but no name had ever been assigned, so a new temporary, informal name is listed that reflects the source of the information (e.g., Dirkes-A).

- Spring Name (proposed) - A descriptive name proposed for the site under the scheme presented in Chapter 8 of this report. The alpha prefix identifies the location as a riverbank spring in one segment of the Hanford Reach, and the following number is the position relative to the HRM system, to the nearest hundredth subdivision.

- HRM (“2k”) - Riverbank spring site relative to the HRM system signposts. Each spring site has been plotted on a base map for the shoreline that includes the locations of HRM signposts, as updated in 1999. The spring position relative to these signposts was determined using proportional-scale dividers to subdivide the distance between each signpost position.

- Segment - Segments defined for convenience in grouping monitoring sites by CERCLA groundwater operable units, groundwater interest areas, or other common characteristic.

- Northing and Easting - Location coordinates in Washington State Plane (South Zone) system; units are meters; North American Datum of 1983.

- Coordinate Quality - Quality of the coordinates, e.g. “surveyed” using global positioning system (GPS) equipment or traditional land survey methods; "estimated” using geographic information system (GIS) maps.

- Coordinate Type/Source - Source for coordinate information. “GPS” refers to global positioning system equipment. "ARCmap” refers to geographic information system coverages for the river, including topography, shorelines, facilities, and the HRM system. This system was used to position riverbank spring sites when the best available information was narrative descriptions found in historical reports. The system (ARCInfo, ${ }^{\mathrm{TM}}$ ESRI Inc, Bellevue, Washington) uses the state plane 
coordinate system, so estimates for coordinates could be read directly from the display. "1:2000map" refers to notes provided on 1:2,000 scale topographic maps during the 1991 field reconnaissance of springs sites by PNL and Westinghouse Hanford Company (WHC) personnel.

- Coordinate Reference - Citation for information on the riverbank spring site.

\subsection{Riverbank Springs: Previous Names}

Table 4.2 is a compendium of various names used to refer to riverbank springs during previous investigations. This table represents the reference database from which judgment calls were made to group riverbank springs together that appear to represent a single location. One record was chosen as the "prime" or host (i.e., those sites listed in previous Table 4.1). The other references to the same site are archived as "secondary" name records.

Table 4.2 provides the metadata necessary to document assigning a common site identifier and descriptive location name to results in the HEIS that represent riverbank spring water samples. Some of the columns in Table 4.2 are same as used in Table 4.1, with exceptions as follows:

- Name as Used in Reference - Name as used in historical report or other reference that includes riverbank spring names.

- Name Reference - Citation for where name has been used, or other source.

- Year of Name Use - Year in which a particular riverbank spring name was used. This was helpful in revealing the evolution of name usage for a particular location.

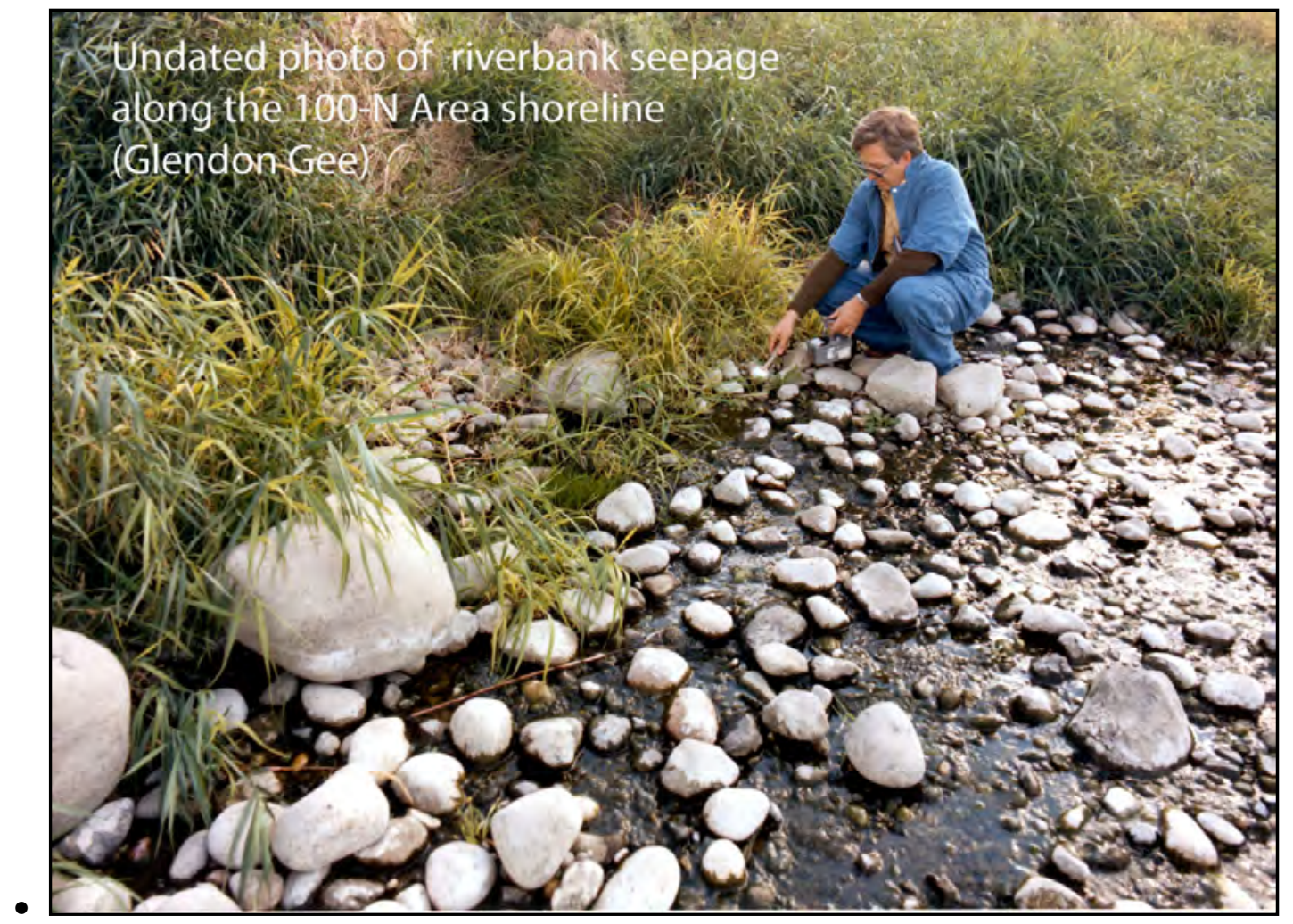

Figure 4.1. Photograph of Riverbank Spring Site 
Table 4.1. Riverbank Springs Observed Along the Hanford Site Shoreline of the Columbia River

\begin{tabular}{|c|c|c|c|c|c|c|c|c|c|}
\hline $\begin{array}{l}\text { Site Identifier } \\
\text { (proposed) }\end{array}$ & $\begin{array}{l}\text { Spring Name } \\
\text { (use in 2009) }\end{array}$ & $\begin{array}{l}\text { Spring Name } \\
\text { (proposed) }\end{array}$ & $\begin{array}{c}\text { HRM } \\
\text { ("2k") }\end{array}$ & Segment $^{1}$ & $\begin{array}{c}\text { Northing } \\
\text { (m-NAD83) }\end{array}$ & $\begin{array}{c}\text { Easting } \\
\text { (m-NAD83) }\end{array}$ & $\begin{array}{c}\text { Coordinate } \\
\text { Quality }^{2}\end{array}$ & $\begin{array}{l}\text { Coordinate } \\
\text { Type/Source }\end{array}$ & Coordinate Reference \\
\hline S1000 & SVB-000-1 & SVB-0000 & 0.000 & VB & & & & & 100-B Pilot Risk Study \\
\hline S1001 & HRM 2.5 & & 2.500 & VB & $146,095.900$ & $562,547.600$ & EST & ARCmap & PNL-7500, Table B.6 \\
\hline S1002 & $3-1 \mathrm{~A}$ & & 3.000 & BC5 & $145,765.900$ & $563,309.500$ & EST & ARCmap & PNL-5289, Table A.1 Narrative \\
\hline S1003 & $3-1 B$ & & 3.050 & BC5 & $145,758.800$ & $563,327.100$ & EST & ARCmap & PNL-5289, Table A.1 Narrative \\
\hline S1004 & $3-2$ & & 3.300 & BC5 & $145,527.100$ & $563,825.700$ & EST & ARCmap & PNL-5289, Table A.1 Narrative \\
\hline S1005 & 3-3 & & 3.410 & BC5 & $145,440.100$ & $564,037.300$ & EST & ARCmap & PNL-5289, Table A.1 Narrative \\
\hline S1006 & $3-4$ & & 3.550 & $\mathrm{BC5}$ & $145,302.500$ & $564,271.000$ & EST & ARCmap & PNL-5289, Table A.1 Narrative \\
\hline S1007 & SB-037-1 & SB-0373 & 3.730 & $\mathrm{BC} 5$ & $145,296.917$ & $564,617.215$ & SURV & GPS & 19981999 combo seeps.xls (GP spr2000) \\
\hline S1008 & SB-038-3 & SB-0375 & 3.750 & $\mathrm{BC} 5$ & $145,278.036$ & $564,662.546$ & SURV & GPS & 19981999 combo seeps.xls (GP spr2000) \\
\hline S1009 & SB-039-2 & SB-0395 & 3.950 & BC5 & $145,369.161$ & $564,951.835$ & SURV & GPS & 19981999 combo seeps.xls (GP spr2000) \\
\hline S1010 & $041-1$ & & 4.100 & BC5 & $145,400.000$ & $565,260.000$ & EST & 1:2000map & WHC-0609, Table A-1 \\
\hline S1011 & $4-1$ & & 4.110 & $\mathrm{BC5}$ & $145,400.000$ & $565,283.000$ & EST & 1:2000map & WHC-0609, Table A-1 \\
\hline S1012 & SB-042-1 & SB-0419 & 4.190 & BC5 & $145,443.000$ & $565,430.000$ & EST & 1:2000map & WHC-0609, Table A-1 \\
\hline S1013 & $047-1$ & & 4.890 & $\mathrm{BC} 5$ & $145,817.000$ & $566,633.000$ & EST & 1:2000map & WHC-0609, Table A-1 \\
\hline S1014 & $5-1$ & & 5.060 & $\mathrm{BC5}$ & $145,908.000$ & $566,920.000$ & EST & 1:2000map & WHC-0609, Table A-1 \\
\hline S1015 & $5-2$ & & 5.290 & BC5 & $146,030.000$ & $567,208.000$ & EST & 1:2000map & WHC-0609, Table A-1 \\
\hline S1016 & $5-3$ & & 5.360 & $\mathrm{BC} 5$ & $146,060.000$ & $567,300.000$ & EST & 1:2000map & WHC-0609, Table A-1 \\
\hline S1017 & $5-4$ & & 5.390 & BC5 & $146,088.000$ & $567,339.000$ & EST & 1:2000map & WHC-0609, Table A-1 \\
\hline S1018 & SK-057-3 & SK-0555 & 5.550 & KR4 & $146,205.197$ & $567,531.527$ & SURV & GPS & 19981999 combo seeps.xls (GP spr2000) \\
\hline S1019 & $5-4 A$ & & 5.560 & KR4 & $146,190.000$ & $567,551.000$ & EST & 1:2000map & WHC-0609, Table A-1 \\
\hline S1020 & $5-6$ & & 5.970 & KR4 & $146,525.000$ & $567,900.000$ & EST & 1:2000map & WHC-0609, Table A-1 \\
\hline S1021 & $6-1$ & & 6.115 & KR4 & $146,635.000$ & $568,138.000$ & EST & 1:2000map & WHC-0609, Table A-1 \\
\hline S1022 & $6-2$ & & 6.125 & KR4 & $146,644.000$ & $568,160.000$ & EST & 1:2000map & WHC-0609, Table A-1 \\
\hline S1023 & $6-3$ & & 6.270 & KR4 & $146,800.000$ & $568,339.000$ & EST & 1:2000map & WHC-0609, Table A-1 \\
\hline S1024 & SK-063-1 & SK-0631 & 6.305 & KR4 & $146,847.084$ & $568,377.710$ & SURV & GPS & 19981999 combo seeps.xls (GP spr2000) \\
\hline S1025 & SK-063-2 & SK-0632 & 6.320 & KR4 & $146,934.800$ & $568,541.600$ & EST & ARCmap & Dirkes91map; prepared for WHC (IT91) \\
\hline S1026 & $7-0$ & & 6.770 & KR4 & $147,250.000$ & $568,986.000$ & EST & 1:2000map & WHC-0609, Table A-1 \\
\hline S1027 & SK-068-1 & SK-0680 & 6.800 & KR4 & $147,318.600$ & $568,982.300$ & EST & ARCmap & Dirkes91map; prepared for WHC (IT91) \\
\hline S1028 & $7-1 \mathrm{~A}$ & & 6.950 & KR4 & $147,500.000$ & $569,121.000$ & EST & 1:2000map & WHC-0609, Table A-1 \\
\hline S1029 & $7-1 \mathrm{~B}$ & & 7.000 & KR4 & $147,580.200$ & $569,180.300$ & EST & ARCmap & PNL-5289, Table A.1 Narrative \\
\hline $\mathrm{S} 1030$ & 071-1 & & 7.060 & KR4 & $147,630.000$ & $569,260.000$ & EST & 1:2000map & WHC-0609, Table A-1 \\
\hline S1031 & IT91-A & & 7.250 & KR4 & $147,821.900$ & $569,436.300$ & EST & ARCmap & Mahood91map; 1:2K reconnaissance \\
\hline S1032 & $7-2$ & & 7.290 & KR4 & $147,870.000$ & $569,475.000$ & EST & 1:2000map & WHC-0609, Table A-1 \\
\hline S1033 & $7-3$ & & 7.330 & KR4 & $147,920.000$ & $569,527.000$ & EST & 1:2000map & WHC-0609, Table A-1 \\
\hline S1034 & k-nonumb & SK-0738 & 7.380 & KR4 & $147,993.950$ & $569,559.900$ & SURV & GPS & PNNL10/01/03 AQST recon. \\
\hline S1035 & Dirkes-C & & 7.440 & KR4 & $148,025.600$ & $569,641.900$ & EST & ARCmap & Dirkes91map; prepared for WHC (IT91) \\
\hline S1036 & Dirkes-D & & 7.520 & KR4 & $148,111.900$ & $569,719.600$ & EST & ARCmap & Dirkes91map; prepared for WHC (IT91) \\
\hline
\end{tabular}


Table 4.1. (contd)

\begin{tabular}{|c|c|c|c|c|c|c|c|c|c|}
\hline $\begin{array}{l}\text { Site Identifier } \\
\text { (proposed) }\end{array}$ & $\begin{array}{l}\text { Spring Name } \\
\text { (use in 2009) }\end{array}$ & $\begin{array}{l}\text { Spring Name } \\
\text { (proposed) }\end{array}$ & $\begin{array}{c}\text { HRM } \\
\text { ("2k") }\end{array}$ & Segment $^{1}$ & $\begin{array}{c}\text { Northing } \\
\text { (m-NAD83) }\end{array}$ & $\begin{array}{c}\text { Easting } \\
\text { (m-NAD83) }\end{array}$ & $\begin{array}{c}\text { Coordinate } \\
\text { Quality }^{2}\end{array}$ & $\begin{array}{l}\text { Coordinate } \\
\text { Type/Source }\end{array}$ & Coordinate Reference \\
\hline S1037 & IT91-B & & 7.560 & KR4 & $148,159.300$ & $569,770.800$ & EST & ARCmap & Mahood91map; 1:2K reconnaissance \\
\hline S1038 & SK-077-1 & SK-0763 & 7.630 & KR4 & $148,242.700$ & $569,849.400$ & EST & ARCmap & Mahood91map; 1:2K reconnaissance \\
\hline S1039 & IT91-D & & 7.710 & KR4 & $148,314.700$ & $569,943.300$ & EST & ARCmap & Mahood91map; 1:2K reconnaissance \\
\hline S1040 & IT91-E & & 7.740 & KR4 & $148,341.300$ & $569,984.100$ & EST & ARCmap & Mahood91map; 1:2K reconnaissance \\
\hline S1041 & IT91-F & & 7.925 & KR4 & $148,520.400$ & $570,185.000$ & EST & ARCmap & Mahood91map; 1:2K reconnaissance \\
\hline S1042 & $079-1$ & & 8.030 & KR4 & $148,643.000$ & $570,287.000$ & EST & 1:2000map & WHC-0609, Table A-1 \\
\hline S1043 & 080-1 & & 8.080 & NR2 & $148,709.000$ & $570,360.000$ & EST & 1:2000map & WHC-0609, Table A-1 \\
\hline S1044 & SK-082-2 & SK-0811 & 8.110 & NR2 & $148,792.099$ & $570,370.443$ & SURV & GPS & 19981999 combo seeps.xls (GP spr2000) \\
\hline S1045 & Dirkes-E & & 8.380 & NR2 & $149,131.200$ & $570,727.900$ & EST & ARCmap & Dirkes91map; prepared for WHC (IT91) \\
\hline S1046 & $8-10$ & & 8.660 & NR2 & $149,540.000$ & $571,018.000$ & EST & 1:2000map & WHC-0609, Table A-1 \\
\hline S1047 & $8-11$ & & 8.700 & NR2 & $149,600.000$ & $571,073.000$ & EST & 1:2000map & WHC-0609, Table A-1 \\
\hline S1048 & Dirkes-F & & 8.760 & NR2 & $149,682.900$ & $571,135.900$ & EST & ARCmap & Dirkes91map; prepared for WHC (IT91) \\
\hline S1049 & $8-12$ & & 8.820 & NR2 & $149,791.000$ & $571,188.400$ & EST & ARCmap & PNL-5289, Table A.1 Narrative \\
\hline S1050 & SN-089-1 & SN-0890 & 8.900 & NR2 & $149,920.000$ & $571,300.000$ & EST & 1:2000map & WHC-0609, Table A-1 \\
\hline S1051 & SN-092-2 & SN-0906 & 9.060 & NR2 & $150,150.000$ & $571,465.000$ & EST & 1:2000map & DOE/RL-92-12, App. C \\
\hline S1052 & $\mathrm{SN}-092-3$ & SN-0908 & 9.080 & NR2 & $150,170.000$ & $571,480.000$ & EST & 1:2000map & DOE/RL-92-12, App. C \\
\hline S1053 & SN-093-1 & SN-0915 & 9.150 & NR2 & $150,256.800$ & $571,552.100$ & EST & ARCmap & PNL-5289, Table A.1 Narrative \\
\hline S1054 & $9-2$ & & 9.175 & NR2 & $150,288.000$ & $571,575.000$ & EST & 1:2000map & WHC-0609, Table A-1 \\
\hline S1055 & $9-3$ & & 9.225 & NR2 & $150,350.000$ & $571,618.000$ & EST & 1:2000map & WHC-0609, Table A-1 \\
\hline S1056 & $9-4$ & & 9.250 & NR2 & $150,391.900$ & $571,640.700$ & EST & ARCmap & Dirkes91map; prepared for WHC (IT91) \\
\hline S1057 & Dirkes-H & & 9.275 & NR2 & $150,424.800$ & $571,662.800$ & EST & ARCmap & Dirkes91map; prepared for WHC (IT91) \\
\hline S1058 & SN-094-1 & SN-0931 & 9.310 & NR2 & $150,470.200$ & $571,675.900$ & SURV & GPS & SESP Trimble sub-meter GPS, 3/30/04 \\
\hline S1059 & SN-NS8-13 & SN-0933 & 9.325 & NR2 & $150,499.700$ & $571,686.400$ & SURV & GPS & SESP Trimble sub-meter GPS, 3/30/04 \\
\hline S1060 & Dirkes-I & SN-0936 & 9.360 & NR2 & $150,541.700$ & $571,728.700$ & SURV & GPS & SESP Trimble sub-meter GPS, 3/30/04 \\
\hline S1061 & Dirkes-J & & 9.410 & NR2 & $150,608.900$ & $571,798.800$ & EST & ARCmap & Dirkes91map; prepared for WHC (IT91) \\
\hline S1062 & Dirkes-K & & 9.490 & NR2 & $150,718.100$ & $571,872.900$ & EST & ARCmap & Dirkes91map; prepared for WHC (IT91) \\
\hline S1063 & $095-1$ & & 9.500 & NR2 & $150,721.100$ & $571,875.900$ & EST & 1:2000map & WHC-0609, Table A-1 \\
\hline S1064 & Dirkes-M & & 9.570 & NR2 & $150,953.300$ & $572,052.400$ & EST & ARCmap & Dirkes91map; prepared for WHC (IT91) \\
\hline S1065 & Dirkes-N & & 9.625 & NR2 & $151,028.200$ & $572,097.400$ & EST & ARCmap & Dirkes91map; prepared for WHC (IT91) \\
\hline S1066 & SD-098-1 & & 9.700 & NR2 & $151,132.971$ & $572,173.329$ & SURV & GPS & 19981999 combo seeps.xls (GP spr2000) \\
\hline S1067 & Dirkes-O & SN-0986 & 9.860 & NR2 & $151,204.600$ & $572,244.100$ & EST & ARCmap & Dirkes91map; prepared for WHC (IT91) \\
\hline S1068 & Dirkes-L & & 9.930 & NR2 & $150,896.100$ & $572,010.400$ & EST & ARCmap & Dirkes91map; prepared for WHC (IT91) \\
\hline S1069 & Dirkes-P & SN-0995 & 9.950 & NR2 & $151,321.500$ & $572,341.100$ & EST & ARCmap & Dirkes91map; prepared for WHC (IT91) \\
\hline S1070 & Dirkes-Q & & 10.030 & HR3D & $151,420.800$ & $572,432.000$ & EST & ARCmap & Dirkes91map; prepared for WHC (IT91) \\
\hline S1071 & SD-102-1 & SD-1024 & 10.240 & HR3D & $151,682.899$ & $572,633.693$ & SURV & GPS & 19981999 combo seeps.xls (GP spr2000) \\
\hline S1072 & SD-105-1 & SD-1050 & 10.500 & HR3D & $151,958.225$ & $572,919.626$ & SURV & GPS & ERC(9\&10/96); GPS-DATA.xls; GPS-CONV.db \\
\hline S1073 & SD-107-1 & SD-1068 & 10.680 & HR3D & $152,168.980$ & $573,143.112$ & SURV & GPS & ERC(9\&10/96); GPS-DATA.xls; GPS-CONV.db \\
\hline
\end{tabular}


Table 4.1. (contd)

\begin{tabular}{|c|c|c|c|c|c|c|c|c|c|}
\hline $\begin{array}{l}\text { Site Identifier } \\
\text { (proposed) }\end{array}$ & $\begin{array}{l}\text { Spring Name } \\
\text { (use in 2009) }\end{array}$ & $\begin{array}{l}\text { Spring Name } \\
\text { (proposed) }\end{array}$ & $\begin{array}{c}\text { HRM } \\
\text { ("2k") }\end{array}$ & Segment $^{1}$ & $\begin{array}{c}\text { Northing } \\
\text { (m-NAD83) }\end{array}$ & $\begin{array}{c}\text { Easting } \\
\text { (m-NAD83) }\end{array}$ & $\begin{array}{c}\text { Coordinate } \\
\text { Quality }^{2}\end{array}$ & $\begin{array}{c}\text { Coordinate } \\
\text { Type/Source }\end{array}$ & Coordinate Reference \\
\hline S1074 & SD-108-1 & SD-1081 & 10.810 & HR3D & $152,277.853$ & $573,310.364$ & SURV & GPS & ERC(9\&10/96); GPS-DATA.xls; GPS-CONV.db \\
\hline S1075 & SD-109-1 & SD-1088 & 10.875 & HR3D & $152,322.063$ & $573,399.195$ & SURV & GPS & ERC(9\&10/96); GPS-DATA.xls; GPS-CONV.db \\
\hline S1076 & $109-1 \mathrm{c}$ & & 10.925 & HR3D & $152,374.654$ & $573,471.976$ & SURV & GPS & ERC(9\&10/96); GPS-DATA.xls; GPS-CONV.db \\
\hline S1077 & SD-110-1 & SD-1103 & 11.025 & HR3D & $152,482.636$ & $573,605.920$ & SURV & GPS & 19981999 combo seeps.xls (GP spr2000) \\
\hline S1078 & SD-110-2 & SD-1112 & 11.115 & HR3D & $152,563.685$ & $573,712.753$ & SURV & GPS & ERC(9\&10/96); GPS-DATA.xls; GPS-CONV.db \\
\hline S1079 & SH-144-1 & SH-1422 & 14.220 & HR3H & $153,838.700$ & $576,992.665$ & SURV & GPS & 19981999 combo seeps.xls (GP spr2000) \\
\hline S1080 & Dirkes-R & & 14.280 & $\mathrm{HR} 3 \mathrm{H}$ & $153,805.700$ & $577,067.700$ & EST & ARCmap & Dirkes91map; prepared for WHC (IT91) \\
\hline S1081 & SH-145-1 & SH-1438 & 14.380 & HR3H & $153,712.996$ & $577,184.064$ & SURV & GPS & 19981999 combo seeps.xls (GP spr2000) \\
\hline S1082 & SH-145-2 & SH-1444 & 14.440 & HR3H & $153,666.605$ & $577,257.223$ & SURV & GPS & ERC(9\&10/96); GPS-DATA.xls; GPS-CONV.db \\
\hline S1083 & Dirkes-S & & 14.525 & $\mathrm{HR} 3 \mathrm{H}$ & $153,621.800$ & $577,360.000$ & EST & ARCmap & Dirkes91map; prepared for WHC (IT91) \\
\hline S1084 & $14-5$ & & 14.560 & HR3H & $153,580.000$ & $577,400.000$ & EST & 1:2000map & WHC-0609, Table A-1 \\
\hline S1085 & $147-1$ & & 14.760 & HR3H & $153,420.000$ & $577,625.000$ & EST & 1:2000map & WHC-0609, Table A-1 \\
\hline S1086 & SH-150-1 & SH-1502 & 15.020 & HR3H & $153,155.016$ & $577,891.104$ & SURV & GPS & 19981999 combo seeps.xls (GP spr2000) \\
\hline S1087 & $152-1$ & & 15.060 & HR3H & $153,090.525$ & $577,957.719$ & SURV & GPS & ERC(9\&10/96); GPS-DATA.xls; GPS-CONV.db \\
\hline S1088 & 151 & & 15.115 & $\mathrm{HR} 3 \mathrm{H}$ & $153,023.965$ & $578,016.276$ & SURV & GPS & ERC(9\&10/96); GPS-DATA.xls; GPS-CONV.db \\
\hline S1089 & SH-152-2 & SH-1526 & 15.265 & HR3H & $152,807.372$ & $578,191.397$ & SURV & GPS & 19981999 combo seeps.xls (GP spr2000) \\
\hline S1090 & SH-152-4 & SH-1532 & 15.315 & $\mathrm{HR} 3 \mathrm{H}$ & $152,716.174$ & $578,220.933$ & SURV & GPS & ERC(9\&10/96); GPS-DATA.xls; GPS-CONV.db \\
\hline S1091 & SH-153-1 & SH-1534 & 15.340 & $\mathrm{HR} 3 \mathrm{H}$ & $152,679.045$ & $578,255.479$ & SURV & GPS & 19981999 combo seeps.xls (GP spr2000) \\
\hline S1092 & $158-1$ & & 15.600 & HR3H & $152,311.000$ & $578,552.000$ & EST & 1:2000map & WHC-0609, Table A-1 \\
\hline S1093 & $178-1$ & & 17.400 & $\mathrm{HR} 3 \mathrm{H}$ & $149,883.000$ & $579,459.900$ & EST & ARCmap & unknown \\
\hline S1094 & $18-0$ & & 17.975 & FR3 & $149,066.000$ & $579,837.000$ & EST & 1:2000map & WHC-0609, Table A-1 \\
\hline S1095 & $18-1$ & & 18.110 & FR3 & $148,936.000$ & $580,018.000$ & EST & 1:2000map & WHC-0609, Table A-1 \\
\hline S1096 & $18-2$ & & 18.620 & FR3 & $148,375.000$ & $580,672.000$ & EST & 1:2000map & WHC-0609, Table A-1 \\
\hline S1097 & SF-187-1 & SF-1880 & 18.800 & FR3 & $148,193.412$ & $580,927.850$ & SURV & GPS & 19981999 combo seeps.xls (GP spr2000) \\
\hline S1098 & 18.3 & & 18.840 & FR3 & $148,156.394$ & $580,975.221$ & SURV & GPS & 19981999 combo seeps.xls (GP spr2000) \\
\hline S1099 & SF-189-1 & SF-1890 & 18.900 & FR3 & $148,099.800$ & $581,062.800$ & EST & ARCmap & unknown \\
\hline S1100 & SF-190-1 & & 18.985 & FR3 & $148,003.540$ & $581,171.724$ & SURV & GPS & 19981999 combo seeps.xls (GP spr2000) \\
\hline S1101 & SF-190-4 & SF-1903 & 19.030 & FR3 & $147,940.000$ & $581,230.000$ & EST & 1:2000map & DOE/RL-92-12, App. C \\
\hline S1102 & SF-205-1 & SF-1953 & 19.530 & FR3 & $146,183.200$ & $582,158.900$ & EST & ARCmap & unknown \\
\hline S1103 & SF-207-1 & SF-2132 & 21.320 & FR3 & $145,468.608$ & $582,691.406$ & SURV & GPS & 19981999 combo seeps.xls (GP spr2000) \\
\hline S1104 & SF-210-1 & SF-2141 & 21.410 & FR3 & $145,370.658$ & $582,751.292$ & SURV & GPS & 19981999 combo seeps.xls (GP spr2000) \\
\hline S1105 & SF-211-1 & SF-2164 & 21.640 & FR3 & $145,130.000$ & $582,864.000$ & EST & 1:2000map & DOE/RL-92-12, App. C \\
\hline S1106 & SF-213-1 & SF-2190 & 21.900 & FR3 & $144,813.000$ & $582,962.000$ & EST & 1:2000map & DOE/RL-92-12, App. C \\
\hline S1107 & SF-216-1 & SF-2230 & 22.300 & FR3 & $144,317.000$ & $583,132.000$ & EST & 1:2000map & DOE/RL-92-12, App. C \\
\hline S1108 & $22-2$ & & 22.410 & FR3 & $144,166.000$ & $583,193.000$ & EST & 1:2000map & WHC-0609, Table A-1 \\
\hline S1109 & $22-3$ & & 22.570 & FR3 & $143,935.000$ & $583,267.000$ & EST & 1:2000map & WHC-0609, Table A-1 \\
\hline S1110 & $22-4$ & & 22.690 & FR3 & $143,764.000$ & $583,303.000$ & EST & 1:2000map & WHC-0609, Table A-1 \\
\hline
\end{tabular}


Table 4.1. (contd)

\begin{tabular}{|c|c|c|c|c|c|c|c|c|c|}
\hline $\begin{array}{l}\text { Site Identifier } \\
\text { (proposed) }\end{array}$ & $\begin{array}{l}\text { Spring Name } \\
\text { (use in 2009) }\end{array}$ & $\begin{array}{l}\text { Spring Name } \\
\text { (proposed) }\end{array}$ & $\begin{array}{c}\text { HRM } \\
(" 2 \mathrm{k})\end{array}$ & Segment $^{1}$ & $\begin{array}{c}\text { Northing } \\
\text { (m-NAD83) }\end{array}$ & $\begin{array}{c}\text { Easting } \\
\text { (m-NAD83) }\end{array}$ & $\begin{array}{c}\text { Coordinate } \\
\text { Quality }^{2}\end{array}$ & $\begin{array}{c}\text { Coordinate } \\
\text { Type/Source }\end{array}$ & Coordinate Reference \\
\hline S1111 & $228-1$ & & 22.860 & FR3 & $143,533.000$ & $583,364.000$ & EST & 1:2000map & WHC-0609, Table A-1 \\
\hline S1112 & $23-1$ & & 23.110 & HTS & $143,167.000$ & $583,511.000$ & EST & 1:2000map & WHC-0609, Table A-1 \\
\hline S1113 & 23-2 & & 23.375 & HTS & $142,789.000$ & $583,693.000$ & EST & 1:2000map & WHC-0609, Table A-1 \\
\hline S1114 & $23-3$ & & 23.615 & HTS & $142,448.000$ & $583,876.000$ & EST & 1:2000map & WHC-0609, Table A-1 \\
\hline S1115 & $23-4 \mathrm{~A}$ & & 23.700 & HTS & $142,358.000$ & $583,986.000$ & EST & 1:2000map & WHC-0609, Table A-1 \\
\hline S1116 & $238-2$ & & 23.800 & HTS & $142,143.000$ & $584,192.000$ & EST & 1:2000map & WHC-0609, Table A-1 \\
\hline S1117 & $23-4 \mathrm{~B}$ & & 23.850 & HTS & $142,212.000$ & $584,181.000$ & EST & 1:2000map & WHC-0609, Table A-1 \\
\hline S1118 & $241-1$ & ST-2406 & 24.060 & HTS & $142,006.330$ & $584,429.830$ & EST & ARCmap & WHC-0609, Table A-1 \\
\hline S1119 & $247-1$ & ST-2472 & 24.720 & HTS & $140,949.130$ & $584,855.390$ & EST & ARCmap & WHC-0609, Table A-1 \\
\hline $\mathrm{S} 1120$ & $25-1 S$ & & 24.750 & HTS & $140,924.000$ & $584,876.000$ & EST & 1:2000map & WHC-0609, Table A-1 \\
\hline S1121 & $25-2 S$ & & 24.840 & HTS & $140,838.000$ & $584,986.000$ & EST & 1:2000map & WHC-0609, Table A-1 \\
\hline S1122 & $25-5 S$ & & 25.220 & HTS & $140,345.600$ & $585,462.100$ & EST & ARCmap & PNL-5289, Table A.1 Narrative \\
\hline S1123 & $25-1$ & & 25.240 & HTS & $140,271.000$ & $585,473.000$ & EST & 1:2000map & WHC-0609, Table A-1 \\
\hline S1124 & $25-2$ & & 25.310 & HTS & $140,192.000$ & $585,632.000$ & EST & 1:2000map & WHC-0609, Table A-1 \\
\hline S1125 & $25-3$ & & 25.380 & HTS & $140,070.000$ & $585,729.000$ & EST & 1:2000map & WHC-0609, Table A-1 \\
\hline S1126 & $25-4$ & & 25.740 & HTS & $139,597.100$ & $586,275.300$ & EST & ARCmap & PNL-5289, Table A.1 Narrative \\
\hline S1127 & $25-5$ & & 25.760 & HTS & $139,582.000$ & $586,339.000$ & EST & 1:2000map & WHC-0609, Table A-1 \\
\hline $\mathrm{S} 1128$ & $25-6$ & & 25.780 & HTS & $139,541.400$ & $586,370.300$ & EST & ARCmap & PNL-5289, Table A.1 Narrative \\
\hline S1129 & $26-1$ & & 26.200 & HTS & $139,033.900$ & $586,910.600$ & EST & ARCmap & PNL-5289, Table A.1 Narrative \\
\hline $\mathrm{S} 1130$ & $26-2$ & & 26.240 & HTS & $138,994.600$ & $586,979.300$ & EST & ARCmap & PNL-5289, Table A.1 Narrative \\
\hline S1131 & $26-3$ & & 26.600 & HTS & $138,722.800$ & $587,421.400$ & EST & ARCmap & PNL-5289, Table A.1 Narrative \\
\hline $\mathrm{S} 1132$ & $26-4$ & & 26.880 & HTS & $138,441.200$ & $587,745.500$ & EST & ARCmap & PNL-5289, Table A.1 Narrative \\
\hline $\mathrm{S} 1133$ & $27-1$ & & 27.070 & HTS & $138,293.900$ & $587,974.800$ & EST & ARCmap & PNL-5289, Table A.1 Narrative \\
\hline S1134 & $27-2$ & & 27.250 & HTS & $138,162.900$ & $588,184.300$ & EST & ARCmap & PNL-5289, Table A.1 Narrative \\
\hline S1135 & $27-3$ & & 27.500 & HTS & $137,976.300$ & $588,456.100$ & EST & ARCmap & PNL-5289, Table A.1 Narrative \\
\hline S1136 & SHTS-UR28-2 & ST-2779 & 27.790 & HTS & $137,809.349$ & $588,812.762$ & SURV & GPS & 19981999 combo seeps.xls (GP spr2000) \\
\hline S1137 & SHTS-28-2 & ST-2810 & 28.100 & HTS & $137,605.364$ & $589,190.770$ & SURV & GPS & 19981999 combo seeps.xls (GP spr2000) \\
\hline S1138 & SHTS-DR28-2 & ST-2829 & 28.290 & HTS & $137,453.263$ & $589,440.686$ & SURV & GPS & 19981999 combo seeps.xls (GP spr2000) \\
\hline S1139 & $28-4$ & & 28.660 & HTS & $137,079.100$ & $589,867.400$ & EST & ARCmap & PNL-5289, Table A.1 Narrative \\
\hline S1140 & $28-5$ & & 28.875 & HTS & $136,811.320$ & $590,047.730$ & EST & ARCmap & PNL-5289, Table C.1 Results \\
\hline S1141 & $29-0$ & & 29.050 & HTS & $136,623.960$ & $590,240.440$ & EST & ARCmap & PNL-5289, Table C.1 Results \\
\hline S1142 & $25-4 S$ & & 29.090 & HTS & $140,573.400$ & $585,291.300$ & EST & ARCmap & PNL-5289, Table A.1 Narrative \\
\hline S1143 & $29-1$ & & 29.240 & PO1 & $136,394.700$ & $590,424.000$ & EST & ARCmap & PNL-5289, Table A.1 Narrative \\
\hline S1144 & $29-2$ & & 29.500 & $\mathrm{PO1}$ & $136,091.300$ & $590,696.900$ & EST & ARCmap & PNL-5289, Table A.1 Narrative \\
\hline S1145 & $29-3$ & & 29.750 & $\mathrm{PO1}$ & $135,752.600$ & $590,901.100$ & EST & ARCmap & PNL-5289, Table A.1 Narrative \\
\hline S1146 & $30-0$ & & 30.040 & $\mathrm{PO1}$ & $135,313.200$ & $591,120.800$ & EST & ARCmap & PNL-5289, Table A.1 Narrative \\
\hline S1147 & $30-1$ & & 30.120 & PO1 & $135,168.400$ & $591,162.200$ & EST & ARCmap & PNL-5289, Table A.1 Narrative \\
\hline
\end{tabular}


Table 4.1. (contd)

\begin{tabular}{|c|c|c|c|c|c|c|c|c|c|c|}
\hline \multirow{38}{*}{$\stackrel{+\infty}{\infty}$} & $\begin{array}{l}\text { Site Identifier } \\
\text { (proposed) }\end{array}$ & $\begin{array}{l}\text { Spring Name } \\
\text { (use in 2009) }\end{array}$ & $\begin{array}{l}\text { Spring Name } \\
\text { (proposed) }\end{array}$ & $\begin{array}{c}\text { HRM } \\
\text { ("2k") }\end{array}$ & Segment $^{1}$ & $\begin{array}{c}\text { Northing } \\
\text { (m-NAD83) }\end{array}$ & $\begin{array}{c}\text { Easting } \\
\text { (m-NAD83) }\end{array}$ & $\begin{array}{l}\text { Coordinate } \\
\text { Quality }^{2}\end{array}$ & $\begin{array}{c}\text { Coordinate } \\
\text { Type/Source }\end{array}$ & Coordinate Reference \\
\hline & S1148 & $30-2$ & & 30.940 & $\mathrm{PO} 1$ & $134,108.500$ & $592,359.100$ & EST & ARCmap & PNL-5289, Table A.1 Narrative \\
\hline & S1149 & $31-1$ & & 31.000 & PO1 & $134,059.400$ & $592,447.000$ & EST & ARCmap & PNL-5289, Table A.1 Narrative \\
\hline & S1150 & $31-2$ & & 31.300 & PO1 & $133,702.600$ & $593,013.100$ & EST & ARCmap & PNL-5289, Table A.1 Narrative \\
\hline & S1151 & $31-3$ & & 31.495 & PO1 & $133,436.400$ & $593,344.100$ & EST & ARCmap & PNL-5289, Table A.1 Narrative \\
\hline & S1152 & $31-4$ & & 31.505 & PO1 & $133,413.100$ & $593,356.900$ & EST & ARCmap & PNL-5289, Table A.1 Narrative \\
\hline & S1153 & $31-5$ & & 31.600 & PO1 & $133,229.500$ & $593,455.200$ & EST & ARCmap & PNL-5289, Table A.1 Narrative \\
\hline & S1154 & $32-0$ & & 32.500 & PO1 & $131,565.450$ & $594,224.000$ & EST & ARCmap & PNL-5289, Table C.1 Results \\
\hline & S1155 & $33-1$ & & 33.000 & PO1 & $130,799.500$ & $594,406.500$ & EST & ARCmap & PNL-5289, Table A.1 Narrative \\
\hline & S1156 & $34-1$ & & 34.000 & PO1 & $129,672.400$ & $594,520.300$ & EST & ARCmap & PNL-5289, Table A.1 Narrative \\
\hline & S1157 & $34-2$ & & 34.900 & PO1 & $128,335.900$ & $594,670.200$ & EST & ARCmap & PNL-5289, Table A.1 Narrative \\
\hline & S1158 & $34-3$ & & 34.925 & PO1 & $128,294.600$ & $594,677.900$ & EST & ARCmap & PNL-5289, Table A.1 Narrative \\
\hline & S1159 & $34-4$ & & 34.940 & PO1 & $128,268.700$ & $594,693.500$ & EST & ARCmap & PNL-5289, Table A.1 Narrative \\
\hline & S1160 & $35-1$ & & 35.600 & PO1 & $127,304.500$ & $594,851.200$ & EST & ARCmap & PNL-5289, Table A.1 Narrative \\
\hline & S1161 & $35-2$ & & 36.000 & PO1 & $126,673.700$ & $594,913.200$ & EST & ARCmap & PNL-5289, Table A.1 Narrative \\
\hline & S1162 & $36-1$ & & 36.740 & PO1 & $125,409.600$ & $594,796.900$ & EST & ARCmap & PNL-5289, Table A.1 Narrative \\
\hline & S1163 & $37-1$ & & 37.100 & PO1 & $124,874.500$ & $594,543.600$ & EST & ARCmap & PNL-5289, Table A.1 Narrative \\
\hline & S1164 & $37-2$ & & 37.500 & PO1 & $124,308.300$ & $594,499.600$ & EST & ARCmap & PNL-5289, Table A.1 Narrative \\
\hline & S1165 & $38-1$ & & 38.250 & PO1 & $123,150.200$ & $594,458.300$ & EST & ARCmap & PNL-5289, Table A.1 Narrative \\
\hline & S1166 & $38-10$ & & 38.850 & PO1 & $122,100.600$ & $594,509.900$ & EST & ARCmap & PNL-5289, Table A.1 Narrative \\
\hline & S1167 & $39-1$ & & 39.240 & PO1 & $121,392.300$ & $594,564.200$ & EST & ARCmap & PNL-5289, Table A.1 Narrative \\
\hline & S1168 & $40-1$ & & 40.000 & PO1 & $119,996.300$ & $594,727.100$ & EST & ARCmap & PNL-5289, Table A.1 Narrative \\
\hline & S1169 & $40-2$ & & 40.020 & PO1 & $119,955.000$ & $594,721.900$ & EST & ARCmap & PNL-5289, Table A.1 Narrative \\
\hline & S1170 & Spr-1 & S3-4076 & 40.760 & 3FF5 & $118,630.064$ & $594,482.956$ & SURV & Landsurv & $\operatorname{KAISER}(4 / 3 / 92)$ \\
\hline & S1171 & Spr-2 & S3-4089 & 40.890 & 3FF5 & $118,400.979$ & $594,450.202$ & SURV & Landsurv & $\operatorname{KAISER}(4 / 3 / 92)$ \\
\hline & S1172 & Spr-3 & S3-4126 & 41.260 & 3FF5 & $117,861.591$ & $594,365.433$ & SURV & Landsurv & KAISER(4/3/92) \\
\hline & S1173 & Spr-4 & S3-4132 & 41.325 & 3FF5 & $117,776.349$ & $594,345.099$ & SURV & Landsurv & KAISER(4/3/92) \\
\hline & S1174 & $41-1$ & & 41.500 & 3FF5 & $117,550.800$ & $594,326.400$ & EST & ARCmap & PNL-5289, Table A.1 Narrative \\
\hline & S1175 & Spr-5 & S3-4173 & 41.730 & $3 F F 5$ & $117,249.844$ & $594,327.721$ & SURV & Landsurv & KAISER(4/3/92) \\
\hline & S1176 & Spr-6 & S3-4186 & 41.865 & 3FF5 & $117,082.551$ & $594,338.230$ & SURV & Landsurv & KAISER(4/3/92) \\
\hline & S1177 & $42-1$ & & 42.000 & $3 F F 5$ & $116,904.500$ & $594,378.100$ & EST & ARCmap & PNL-5289, Table A.1 Narrative \\
\hline & S1178 & S3-42-2 & S3-4211 & 42.105 & 3FF5 & $116,696.501$ & $594,392.078$ & SURV & Landsurv & $\operatorname{KAISER}(4 / 3 / 92)$ \\
\hline & S1179 & Spr-8 & S3-4232 & 42.320 & 3FF5 & $116,289.940$ & $594,467.539$ & SURV & Landsurv & $\operatorname{KAISER}(4 / 3 / 92)$ \\
\hline & S1180 & S3-DR42-2 & S3-4236 & 42.365 & 3FF5 & $116,209.879$ & $594,488.883$ & SURV & Landsurv & KAISER(4/3/92) \\
\hline & S1181 & Spr-10 & S3-4243 & 42.430 & $3 \mathrm{FF5}$ & $116,083.503$ & $594,525.323$ & SURV & Landsurv & KAISER(4/3/92) \\
\hline & S1182 & Spr-11 & S3-4267 & 42.670 & $3 F F 5$ & $115,644.462$ & $594,640.955$ & SURV & Landsurv & KAISER(4/3/92) \\
\hline & S1183 & Spr-12 & S3-4306 & 43.060 & $3 \mathrm{FF5}$ & $114,934.799$ & $594,802.480$ & SURV & Landsurv & KAISER(4/3/92) \\
\hline & S1184 & Spr-13 & S3-4318 & 43.180 & $3 F F 5$ & $114,769.187$ & $594,825.660$ & SURV & Landsurv & KAISER(4/3/92) \\
\hline
\end{tabular}


Table 4.1. (contd)

\begin{tabular}{|c|c|c|c|c|c|c|c|c|c|}
\hline $\begin{array}{l}\text { Site Identifier } \\
\text { (proposed) }\end{array}$ & $\begin{array}{l}\text { Spring Name } \\
\text { (use in 2009) }\end{array}$ & $\begin{array}{c}\text { Spring Name } \\
\text { (proposed) }\end{array}$ & $\begin{array}{c}\text { HRM } \\
(" 2 \mathrm{k} ")\end{array}$ & Segment $^{1}$ & $\begin{array}{c}\text { Northing } \\
\text { (m-NAD83) }\end{array}$ & $\begin{array}{c}\text { Easting } \\
\text { (m-NAD83) }\end{array}$ & $\begin{array}{l}\text { Coordinate } \\
\text { Quality }^{2}\end{array}$ & $\begin{array}{l}\text { Coordinate } \\
\text { Type/Source }\end{array}$ & Coordinate Reference \\
\hline S1185 & Spr-14 & S3-4348 & 43.475 & 3FF5 & $114,361.831$ & $594,887.139$ & SURV & Landsurv & KAISER(4/3/92) \\
\hline S1186 & $43-2$ & & 43.775 & 3FF5 & $113,952.300$ & $594,946.800$ & EST & ARCmap & PNL-5289, Table A.1 Narrative \\
\hline S1187 & 43-3 & & 43.860 & $3 \mathrm{FF} 5$ & $113,830.800$ & $594,962.300$ & EST & ARCmap & PNL-5289, Table A.1 Narrative \\
\hline S1188 & SRL-444-1 & & 44.400 & RLD & $113,071.564$ & $595,050.997$ & EST & GPS & SESP field log, Greg Patton, 2001 \\
\hline
\end{tabular}


Table 4.2. Historical Names Used for Riverbank Springs

\begin{tabular}{|c|c|c|c|c|c|c|c|}
\hline $\begin{array}{c}\text { Site } \\
\text { Identifier } \\
\text { (proposed) }\end{array}$ & $\begin{array}{l}\text { Spring Name } \\
\text { (use in 2009) }\end{array}$ & $\begin{array}{c}\text { Spring } \\
\text { Name } \\
\text { (proposed) }\end{array}$ & $\begin{array}{c}\text { HRM } \\
\text { ("2k") }\end{array}$ & Segment $^{1}$ & $\begin{array}{c}\text { Name as Used in } \\
\text { Reference }\end{array}$ & Name Reference & $\begin{array}{c}\text { Year of } \\
\text { Name } \\
\text { Use }^{2}\end{array}$ \\
\hline $\mathrm{S} 1000$ & SVB-000-1 & SVB-0000 & 0.000 & VB & none & none & 2004 \\
\hline \multirow[t]{3}{*}{$\mathrm{S} 1001$} & HRM 2.5 & & 2.500 & VB & HRM 2.5 & PNL-7500 (report) & 1990 \\
\hline & & & & & $025-1$ & WHC-0609, Table A-1 & 1992 \\
\hline & & & & & Large flow & Search-89, Table 5 & 1989 \\
\hline \multirow[t]{2}{*}{$\mathrm{S} 1002$} & $3-1 A$ & & 3.000 & BC5 & $3-1 \mathrm{~A}$ & PNL-5289 (report) & 1984 \\
\hline & & & & & $030-1$ & WHC-0609, Table A-1 & 1992 \\
\hline \multirow[t]{2}{*}{$\mathrm{S} 1003$} & $3-1 B$ & & 3.050 & $\mathrm{BC5}$ & $3-1 B$ & PNL-5289 (report) & 1984 \\
\hline & & & & & 030-2 & WHC-0609, Table A-1 & 1992 \\
\hline \multirow[t]{3}{*}{ S1004 } & $3-2$ & & 3.300 & $\mathrm{BC5}$ & $3-2$ & PNL-5289 (report) & 1984 \\
\hline & & & & & 033-1 & \begin{tabular}{|l|} 
WHC-0609, Table A-1 \\
\end{tabular} & 1992 \\
\hline & & & & & $3-2$ & PNL-7500 (report) & 1990 \\
\hline \multirow[t]{3}{*}{ S1005 } & 3-3 & & 3.410 & BC5 & $3-3$ & PNL-5289 (report) & 1984 \\
\hline & & & & & $033-2$ & WHC-0609, Table A-1 & 1992 \\
\hline & & & & & $035-2$ & WHC-0609, Table A-1 & 1992 \\
\hline \multirow[t]{2}{*}{ S1006 } & $3-4$ & & 3.550 & BC5 & $3-4$ & PNL-5289 (report) & 1984 \\
\hline & & & & & \begin{tabular}{|l|}
$035-1$ \\
\end{tabular} & WHC-0609, Table A-1 & 1992 \\
\hline \multirow[t]{15}{*}{$\mathrm{S} 1007$} & SB-037-1 & SB-0373 & 3.730 & $\mathrm{BC} 5$ & seep371100b & PNNL-98/99 GPS survey & 1999 \\
\hline & & & & & \begin{tabular}{|l|} 
SB-037-1 \\
\end{tabular} & PNNL-13404 (gw report) & 2002 \\
\hline & & & & & seep371100b & PNNL-12088 (env report) & 2000 \\
\hline & & & & & $037-1$ & BC5-RI NPL No. M-15-99-03 & 1999 \\
\hline & & & & & 100-B Spring 37-1 & PNNL-12103 (env schedule) & 1999 \\
\hline & & & & & seep371100b & SESP-SLM-01, S-2.4 & 1999 \\
\hline & & & & & SB-037-1 & BC5-RI 1998 (SAF X99-004) & 1998 \\
\hline & & & & & SB-037-1 & BC5-RI 1997 (SAF N98-002) & 1997 \\
\hline & & & & & SB-037-1 & BC5-RI 1996 (CCN 041996) & 1996 \\
\hline & & & & & $037-1$ & BC5-RI NPL No. M-15-96-07 & 1996 \\
\hline & & & & & 100 B Spring & PNL-1995 GPS survey & 1995 \\
\hline & & & & & $037-1$ & BC5-RI 1993 & 1993 \\
\hline & & & & & 037-1 & WHC-0609, Table A-1 & 1992 \\
\hline & & & & & $037-1$ & DOE/RL-92-12, Appendix F & 1992 \\
\hline & & & & & $3-5$ & PNL-5289 (report) & 1984 \\
\hline \multirow[t]{14}{*}{ S1008 } & SB-038-3 & SB-0375 & 3.750 & $\mathrm{BC} 5$ & seep38.3 & PNNL-98/99 GPS survey & 1999 \\
\hline & & & & & SB-038-3 & PNNL-13404 (gw report) & 2002 \\
\hline & & & & & 100-B Spring 38-3 & PNNL-13418 (env schedule) & 2001 \\
\hline & & & & & 100-b spring 38-3 & PNNL-13230 (env report) & 2000 \\
\hline & & & & & 100-B Spring 38-3 & PNNL-13109 (env schedule) & 2000 \\
\hline & & & & & seep38.3 & PNNL-12088 (env report) & 2000 \\
\hline & & & & & seep38.3 & SESP-SLM-01, S-2.4 & 1999 \\
\hline & & & & & 100-B Spring & PNNL-11803 (env schedule) & 1998 \\
\hline & & & & & 100-B Spring & PNNL-11464 (env schedule) & 1997 \\
\hline & & & & & 100-B Spring & PNNL-10950 (env schedule) & 1996 \\
\hline & & & & & 100-B Spring & PNL-10423 (env schedule) & 1995 \\
\hline & & & & & $038-2$ & WHC-0609, Table A-1 & 1992 \\
\hline & & & & & 038-3 & DOE/RL-92-12, Appendix F & 1992 \\
\hline & & & & & $3-6$ & PNL-5289 (report) & 1984 \\
\hline \multirow[t]{6}{*}{ S1009 } & SB-039-2 & SB-0395 & 3.950 & $\mathrm{BC5}$ & seep39.2100b & PNNL-98/99 GPS survey & 1999 \\
\hline & & & & & SB-039-2 & PNNL-13404 (gw report) & 2002 \\
\hline & & & & & 100-B Spring 39-2 & PNNL-13418 (env schedule) & 2001 \\
\hline & & & & & 100-b spring 39-2 & PNNL-13230 (env report) & 2000 \\
\hline & & & & & 100-B Spring 39-2 & PNNL-13109 (env schedule) & 2000 \\
\hline & & & & & seep39.2100b & PNNL-12088 (env report) & 2000 \\
\hline
\end{tabular}


Table 4.2. (contd)

\begin{tabular}{|c|c|c|c|c|c|c|c|}
\hline $\begin{array}{c}\text { Site } \\
\text { Identifier } \\
\text { (proposed) }\end{array}$ & $\begin{array}{l}\text { Spring Name } \\
\text { (use in 2009) }\end{array}$ & $\begin{array}{c}\text { Spring } \\
\text { Name } \\
\text { (proposed) }\end{array}$ & $\begin{array}{l}\text { HRM } \\
\text { ("2k") }\end{array}$ & Segment $^{1}$ & $\begin{array}{c}\text { Name as Used in } \\
\text { Reference }\end{array}$ & Name Reference & $\begin{array}{c}\text { Year of } \\
\text { Name } \\
\text { Use }\end{array}$ \\
\hline & & & & & $039-2$ & BC5-RI NPL No. M-15-99-03 & 1999 \\
\hline & & & & & seep39.2100b & SESP-SLM-01, S-2.4 & 1999 \\
\hline & & & & & SB-039-2 & BC5-RI 1998 (SAF X99-004) & 1998 \\
\hline & & & & & SB-039-2 & BC5-RI 1997 (SAF N98-002) & 1997 \\
\hline & & & & & SB-039-2 & BC5-RI 1996 (CCN 041996) & 1996 \\
\hline & & & & & $039-2$ & BC5-RI NPL No. M-15-96-07 & 1996 \\
\hline & & & & & 039-1 & WHC-0609, Table A-1 & 1992 \\
\hline & & & & & $039-2$ & DOE/RL-92-12, Appendix F & 1992 \\
\hline & & & & & $4-0$ & PNL-5289 (report) & 1984 \\
\hline \multirow[t]{2}{*}{ S1010 } & 041-1 & & 4.100 & BC5 & 041-1 & WHC-0609, Table A-1 & 1992 \\
\hline & & & & & B breakwater & Search-89, Table 5 & 1989 \\
\hline \multirow[t]{2}{*}{ S1011 } & $4-1$ & & 4.110 & $\mathrm{BC5}$ & $042-1$ & WHC-0609, Table A-1 & 1992 \\
\hline & & & & & 4-1 & PNL-5289 (report) & 1984 \\
\hline \multirow[t]{3}{*}{$\mathrm{S} 1012$} & SB-042-1 & SB-0419 & 4.190 & BC5 & $042-2$ & WHC-0609, Table A-1 & 1992 \\
\hline & & & & & $042-2$ & WHC-0609, Table A-1 & 1992 \\
\hline & & & & & $4-2$ & PNL-5289 (report) & 1984 \\
\hline \multirow[t]{2}{*}{$\mathrm{S} 1013$} & $047-1$ & & 4.890 & BC5 & $047-1$ & WHC-0609, Table A-1 & 1992 \\
\hline & & & & & $\mathrm{d} / \mathrm{s}$ of $\mathrm{B}$ & Search-89, Table 5 & 1989 \\
\hline \multirow[t]{2}{*}{ S1014 } & $5-1$ & & 5.060 & BC5 & $050-1$ & WHC-0609, Table A-1 & 1992 \\
\hline & & & & & $5-1$ & PNL-5289 (report) & 1984 \\
\hline \multirow[t]{2}{*}{$\mathrm{S} 1015$} & $5-2$ & & 5.290 & BC5 & $050-2$ & WHC-0609, Table A-1 & 1992 \\
\hline & & & & & $5-2$ & PNL-5289 (report) & 1984 \\
\hline \multirow[t]{2}{*}{ S1016 } & $5-3$ & & 5.360 & BC5 & $052-1$ & WHC-0609, Table A-1 & 1992 \\
\hline & & & & & $5-3$ & PNL-5289 (report) & 1984 \\
\hline \multirow[t]{2}{*}{ S1017 } & $5-4$ & & 5.390 & $\mathrm{BC5}$ & $052-2$ & WHC-0609, Table A-1 & 1992 \\
\hline & & & & & $5-4$ & PNL-5289 (report) & 1984 \\
\hline \multirow[t]{11}{*}{$\mathrm{S} 1018$} & SK-057-3 & SK-0555 & 5.550 & KR4 & 100k57.3seep & PNNL-98/99 GPS survey & 1999 \\
\hline & & & & & SK-057-3 & PNNL-13404 (gw report) & 2002 \\
\hline & & & & & 100k57.3seep & PNNL-12088 (env report) & 2000 \\
\hline & & & & & 100k57.3seep & SESP-SLM-01, S-2.4 & 1999 \\
\hline & & & & & SK-057-3 & KR4-RI 1997 (SAF C98-009) & 1997 \\
\hline & & & & & SK-057-3 & KR4-RI 1996 (CCN 041996) & 1996 \\
\hline & & & & & $057-3$ & KR4-RI NPL No. 108 & 1996 \\
\hline & & & & & $057-3$ & WHC-0609, Table A-1 & 1992 \\
\hline & & & & & 056-3 & DOE/RL-92-12, Appendix F & 1992 \\
\hline & & & & & $057-3$ & DOE/RL-92-12, Appendix F & 1992 \\
\hline & & & & & $5-5$ & PNL-5289 (report) & 1984 \\
\hline \multirow[t]{2}{*}{ S1019 } & $5-4 \mathrm{~A}$ & & 5.560 & KR4 & 056-1 & WHC-0609, Table A-1 & 1992 \\
\hline & & & & & $5-4 A$ & PNL-5289 (report) & 1984 \\
\hline \multirow[t]{2}{*}{ S1020 } & $5-6$ & & 5.970 & KR4 & 059-1 & WHC-0609, Table A-1 & 1992 \\
\hline & & & & & $5-6$ & PNL-5289 (report) & 1984 \\
\hline \multirow[t]{2}{*}{$\mathrm{S} 1021$} & $6-1$ & & 6.115 & KR4 & 060-1 & WHC-0609, Table A-1 & 1992 \\
\hline & & & & & $6-1$ & PNL-5289 (report) & 1984 \\
\hline \multirow[t]{4}{*}{ S1022 } & $6-2$ & & 6.125 & KR4 & $062-1$ & WHC-0609, Table A-1 & 1992 \\
\hline & & & & & $062-3$ & WHC-0609, Table A-1 & 1992 \\
\hline & & & & & $\mathrm{u} / \mathrm{s}$ of $\mathrm{K}$ tanks & Search-89, Table 5 & 1989 \\
\hline & & & & & $6-2$ & PNL-5289 (report) & 1984 \\
\hline \multirow[t]{4}{*}{ S1023 } & $6-3$ & & 6.270 & KR4 & $062-2$ & WHC-0609, Table A-1 & 1992 \\
\hline & & & & & 063-1 & WHC-0609, Table A-1 & 1992 \\
\hline & & & & & off $d / s$ of $K$ tanks & Search-89, Table 5 & 1989 \\
\hline & & & & & $6-3$ & PNL-5289 (report) & 1984 \\
\hline S1024 & SK-063-1 & SK-0631 & 6.305 & KR4 & 100k6.6seep & PNNL-98/99 GPS survey & 1999 \\
\hline
\end{tabular}


Table 4.2. (contd)

\begin{tabular}{|c|c|c|c|c|c|c|c|}
\hline $\begin{array}{c}\text { Site } \\
\text { Identifier } \\
\text { (proposed) }\end{array}$ & $\begin{array}{l}\text { Spring Name } \\
\text { (use in 2009) }\end{array}$ & $\begin{array}{c}\text { Spring } \\
\text { Name } \\
\text { (proposed) }\end{array}$ & $\begin{array}{c}\text { HRM } \\
\text { ("2k") }\end{array}$ & Segment $^{1}$ & $\begin{array}{c}\text { Name as Used in } \\
\text { Reference }\end{array}$ & Name Reference & $\begin{array}{c}\text { Year of } \\
\text { Name } \\
\text { Use }^{2}\end{array}$ \\
\hline & & & & & SK-063-1 & PNNL-13404 (gw report) & 2002 \\
\hline & & & & & 100-K Spring 63-1 & PNNL-13418 (env schedule) & 2001 \\
\hline & & & & & 100-k spring 63-1 & PNNL-13230 (env report) & 2000 \\
\hline & & & & & 100-K Spring 63-1 & PNNL-13109 (env schedule) & 2000 \\
\hline & & & & & 100k6.6seep & PNNL-12088 (env report) & 2000 \\
\hline & & & & & 100-K Spring 63-1 & PNNL-12103 (env schedule) & 1999 \\
\hline & & & & & 100k6.6seep & SESP-SLM-01, S-2.4 & 1999 \\
\hline & & & & & 100-K Spring & PNNL-11803 (env schedule) & 1998 \\
\hline & & & & & 100-K Spring & PNNL-11464 (env schedule) & 1997 \\
\hline & & & & & 100-K Spring & PNNL-10950 (env schedule) & 1996 \\
\hline & & & & & $100 \mathrm{~K}$ Spring & PNL-1995 GPS survey & 1995 \\
\hline & & & & & 100-K Spring & PNL-10423 (env schedule) & 1995 \\
\hline \multirow[t]{2}{*}{ S1025 } & SK-063-2 & SK-0632 & 6.320 & KR4 & Dirkes-A & SAC/S\&T FY00 data comp & 2000 \\
\hline & & & & & Dirkes-A & SAC/S\&T FY00 data comp & 2000 \\
\hline \multirow[t]{2}{*}{ S1026 } & $7-0$ & & 6.770 & KR4 & 068-1 & WHC-0609, Table A-1 & 1992 \\
\hline & & & & & $7-0$ & PNL-5289 (report) & 1984 \\
\hline \multirow[t]{2}{*}{ S1027 } & SK-068-1 & SK-0680 & 6.800 & KR4 & SK-068-1 & Fall 2002 SESP sampling & 2002 \\
\hline & & & & & Dirkes-B & SAC/S\&T FY00 data comp & 2000 \\
\hline \multirow[t]{2}{*}{$\mathrm{S} 1028$} & $7-1 \mathrm{~A}$ & & 6.950 & KR4 & 069-1 & WHC-0609, Table A-1 & 1992 \\
\hline & & & & & $7-1 \mathrm{~A}$ & PNL-5289 (report) & 1984 \\
\hline \multirow[t]{2}{*}{ S1029 } & $7-1 \mathrm{~B}$ & & 7.000 & KR4 & $7-1 \mathrm{~B}$ & PNL-5289 (report) & 1984 \\
\hline & & & & & 070-1 & WHC-0609, Table A-1 & 1992 \\
\hline \multirow[t]{2}{*}{ S1030 } & 071-1 & & 7.060 & KR4 & 071-1 & WHC-0609, Table A-1 & 1992 \\
\hline & & & & & broad, below $\mathrm{K}$ & Search-89, Table 5 & 1989 \\
\hline S1031 & IT91-A & & 7.250 & KR4 & IT91-A & SAC/S\&T FY00 data comp & 2000 \\
\hline \multirow[t]{2}{*}{ S1032 } & $7-2$ & & 7.290 & KR4 & $072-1$ & WHC-0609, Table A-1 & 1992 \\
\hline & & & & & $7-2$ & PNL-5289 (report) & 1984 \\
\hline \multirow[t]{2}{*}{$\mathrm{S} 1033$} & $7-3$ & & 7.330 & KR4 & $072-2$ & WHC-0609, Table A-1 & 1992 \\
\hline & & & & & $7-3$ & PNL-5289 (report) & 1984 \\
\hline \multirow[t]{5}{*}{$\mathrm{S} 1034$} & k-nonumb & SK-0738 & 7.380 & KR4 & seep100knonumb & PNNL10/01/03 AQST recon. & 2003 \\
\hline & & & & & seep100knonumb & PNNL-12088 (env report) & 2000 \\
\hline & & & & & seep100knonumb & PNNL-98/99 GPS survey & 1999 \\
\hline & & & & & seep100knonumb & SESP-SLM-01, S-2.4 & 1999 \\
\hline & & & & & $7-4$ & PNL-5289 (report) & 1984 \\
\hline S1035 & Dirkes-C & & 7.440 & KR4 & Dirkes-C & SAC/S\&T FY00 data comp & 2000 \\
\hline S1036 & Dirkes-D & & 7.520 & KR4 & Dirkes-D & SAC/S\&T FY00 data comp & 2000 \\
\hline S1037 & IT91-B & & 7.560 & KR4 & IT91-B & SAC/S\&T FY00 data comp & 2000 \\
\hline \multirow[t]{11}{*}{$\mathrm{S} 1038$} & SK-077-1 & SK-0763 & 7.630 & KR4 & IT91-C & SAC/S\&T FY00 data comp & 2000 \\
\hline & & & & & SK-077-1 & PNNL-13404 (gw report) & 2002 \\
\hline & & & & & 100-k spring $77-1$ & PNNL-13230 (env report) & 2000 \\
\hline & & & & & 100-K Spring 77-1 & PNNL-13109 (env schedule) & 2000 \\
\hline & & & & & 100-K Spring 77-1 & PNNL-12103 (env schedule) & 1999 \\
\hline & & & & & SK-077-1 & KR4-RI 1997 (SAF C98-009) & 1997 \\
\hline & & & & & SK-077-1 & KR4-RI NPL No. 108 & 1996 \\
\hline & & & & & 077-1 & KR4-RI 1993 & 1993 \\
\hline & & & & & $077-1$ & WHC-0609, Table A-1 & 1992 \\
\hline & & & & & $074-1$ & DOE/RL-92-12, Appendix F & 1992 \\
\hline & & & & & $077-1$ & DOE/RL-92-12, Appendix F & 1992 \\
\hline S1039 & IT91-D & & 7.710 & KR4 & IT91-D & SAC/S\&T FY00 data comp & 2000 \\
\hline $\mathrm{S} 1040$ & IT91-E & & 7.740 & KR4 & IT91-E & SAC/S\&T FY00 data comp & 2000 \\
\hline $\mathrm{S} 1041$ & IT91-F & & 7.925 & KR4 & IT91-F & SAC/S\&T FY00 data comp & 2000 \\
\hline S1042 & $079-1$ & & 8.030 & KR4 & 079-1 & WHC-0609, Table A-1 & 1992 \\
\hline
\end{tabular}


Table 4.2. (contd)

\begin{tabular}{|c|c|c|c|c|c|c|c|}
\hline $\begin{array}{c}\text { Site } \\
\text { Identifier } \\
\text { (proposed) }\end{array}$ & $\begin{array}{l}\text { Spring Name } \\
\text { (use in 2009) }\end{array}$ & $\begin{array}{c}\text { Spring } \\
\text { Name } \\
\text { (proposed) }\end{array}$ & $\begin{array}{l}\text { HRM } \\
\text { ("2k") }\end{array}$ & Segment $^{1}$ & $\begin{array}{l}\text { Name as Used in } \\
\text { Reference }\end{array}$ & Name Reference & $\begin{array}{l}\text { Year of } \\
\text { Name } \\
\text { Use }\end{array}$ \\
\hline & & & & & onshore pool & Search-89, Table 5 & 1989 \\
\hline S1043 & 080-1 & & 8.080 & NR2 & $080-1$ & WHC-0609, Table A-1 & 1992 \\
\hline \multirow[t]{14}{*}{ S1044 } & SK-082-2 & SK-0811 & 8.110 & NR2 & 100k8.2seep & PNNL-98/99 GPS survey & 1999 \\
\hline & & & & & SK-082-2 & PNNL-13404 (gw report) & 2002 \\
\hline & & & & & 100k8.2seep & PNNL-12088 (env report) & 2000 \\
\hline & & & & & 100k8.2seep & SESP-SLM-01, S-2.4 & 1999 \\
\hline & & & & & SK-082-2 & KR4-RI 1997 (SAF C98-009) & 1997 \\
\hline & & & & & SK-082-2 & KR4-RI 1996 (CCN 041996) & 1996 \\
\hline & & & & & $082-2$ & KR4-RI NPL No. 108 & 1996 \\
\hline & & & & & $082-1$ & WHC-0609, Table A-1 & 1992 \\
\hline & & & & & $082-2$ & WHC-0609, Table A-1 & 1992 \\
\hline & & & & & $080-2$ & DOE/RL-92-12, Appendix F & 1992 \\
\hline & & & & & $082-2$ & DOE/RL-92-12, Appendix F & 1992 \\
\hline & & & & & $\mathrm{u} / \mathrm{s}$ tower at $\mathrm{N}$ & Search-89, Table 5 & 1989 \\
\hline & & & & & $8-1$ & PNL-5289 (report) & 1984 \\
\hline & & & & & $8.25-1$ & PNL-5289 (report) & 1984 \\
\hline \multirow[t]{2}{*}{$\mathrm{S} 1045$} & Dirkes-E & & 8.380 & NR2 & Dirkes-E & SAC/S\&T FY00 data comp & 2000 \\
\hline & & & & & \begin{tabular}{|c|}
$083-2$ \\
\end{tabular} & WHC-0609, Table A-1 & 1992 \\
\hline \multirow[t]{4}{*}{ S1046 } & $8-10$ & & 8.660 & NR2 & 085-1 & WHC-0609, Table A-1 & 1992 \\
\hline & & & & & 086-1 & WHC-0609, Table A-1 & 1992 \\
\hline & & & & & riprap at $\mathrm{N}$ & Search-89, Table 5 & 1989 \\
\hline & & & & & $8-10$ & PNL-5289 (report) & 1984 \\
\hline \multirow[t]{3}{*}{ S1047 } & $8-11$ & & 8.700 & NR2 & $087-1$ & WHC-0609, Table A-1 & 1992 \\
\hline & & & & & entering $N$ chute & Search-89, Table 5 & 1989 \\
\hline & & & & & $8-11$ & PNL-5289 (report) & 1984 \\
\hline \multirow[t]{2}{*}{ S1048 } & Dirkes-F & & 8.760 & NR2 & Dirkes-F & SAC/S\&T FY00 data comp & 2000 \\
\hline & & & & & 088-1 & WHC-0609, Table A-1 & 1992 \\
\hline \multirow[t]{3}{*}{ S1049 } & $8-12$ & & 8.820 & NR2 & $8-12$ & PNL-5289 (report) & 1984 \\
\hline & & & & & $088-2$ & WHC-0609, Table A-1 & 1992 \\
\hline & & & & & Seep spot 1 & UNI-3866, Fig. 1 (report) & 1986 \\
\hline \multirow[t]{9}{*}{$\mathrm{S} 1050$} & SN-089-1 & SN-0890 & 8.900 & NR2 & $089-1$ & WHC-0609, Table A-1 & 1992 \\
\hline & & & & & $100-N$ Spring $199 \mathrm{~N}-46$ & PNNL-13418 (env schedule) & 2001 \\
\hline & & & & & $100-n$ spring $199 n-46$ & PNNL-13230 (env report) & 2000 \\
\hline & & & & & $100-N$ Spring $199 \mathrm{~N}-46$ & PNNL-13109 (env schedule) & 2000 \\
\hline & & & & & $100-N$ Spring $199 \mathrm{~N}-46$ & PNNL-12103 (env schedule) & 1999 \\
\hline & & & & & $090-1$ & WHC-0609, Table A-1 & 1992 \\
\hline & & & & & 090-1 & DOE/RL-92-12, Appendix F & 1992 \\
\hline & & & & & N8T & PNL-7500 (report) & 1990 \\
\hline & & & & & $8-13$ & PNL-5289 (report) & 1984 \\
\hline \multirow[t]{5}{*}{ S1051 } & SN-092-2 & SN-0906 & 9.060 & NR2 & 091-1 & DOE/RL-92-12, Appendix F & 1992 \\
\hline & & & & & 091-1 & WHC-0609, Table A-1 & 1992 \\
\hline & & & & & $092-2$ & WHC-0609, Table A-1 & 1992 \\
\hline & & & & & $092-2$ & DOE/RL-92-12, Appendix F & 1992 \\
\hline & & & & & lower $\mathrm{N}$ springs & Search-89, Table 5 & 1989 \\
\hline \multirow[t]{3}{*}{ S1052 } & SN-092-3 & SN-0908 & 9.080 & NR2 & $092-1$ & DOE/RL-92-12, Appendix F & 1992 \\
\hline & & & & & $092-3$ & WHC-0609, Table A-1 & 1992 \\
\hline & & & & & $092-3$ & DOE/RL-92-12, Appendix F & 1992 \\
\hline \multirow[t]{5}{*}{ S1053 } & SN-093-1 & SN-0915 & 9.150 & NR2 & $9-1$ & PNL-5289 (report) & 1984 \\
\hline & & & & & 093-2 & WHC-0609, Table A-1 & 1992 \\
\hline & & & & & $092-2$ & DOE/RL-92-12, Appendix F & 1992 \\
\hline & & & & & $093-1$ & DOE/RL-92-12, Appendix F & 1992 \\
\hline & & & & & Seep spot 2 & UNI-3866, Fig. 1 (report) & 1986 \\
\hline
\end{tabular}


Table 4.2. (contd)

\begin{tabular}{|c|c|c|c|c|c|c|c|}
\hline $\begin{array}{c}\text { Site } \\
\text { Identifier } \\
\text { (proposed) }\end{array}$ & $\begin{array}{l}\text { Spring Name } \\
\text { (use in 2009) }\end{array}$ & $\begin{array}{c}\text { Spring } \\
\text { Name } \\
\text { (proposed) }\end{array}$ & $\begin{array}{l}\text { HRM } \\
\text { ("2k") }\end{array}$ & Segment $^{1}$ & $\begin{array}{l}\text { Name as Used in } \\
\text { Reference }\end{array}$ & Name Reference & $\begin{array}{l}\text { Year of } \\
\text { Name } \\
\text { Use }\end{array}$ \\
\hline \multirow[t]{5}{*}{ S1054 } & $9-2$ & & 9.175 & NR2 & 093-1 & WHC-0609, Table A-1 & 1992 \\
\hline & & & & & 093-3(sic -4) & WHC-0609, Table A-1 & 1992 \\
\hline & & & & & 093-7 & WHC-0609, Table A-1 & 1992 \\
\hline & & & & & Seep spot 3 & UNI-3866, Fig. 1 (report) & 1986 \\
\hline & & & & & $9-2$ & PNL-5289 (report) & 1984 \\
\hline \multirow[t]{2}{*}{ S1055 } & $9-3$ & & 9.225 & NR2 & $093-3$ & WHC-0609, Table A-1 & 1992 \\
\hline & & & & & $9-3$ & PNL-5289 (report) & 1984 \\
\hline \multirow[t]{4}{*}{ S1056 } & $9-4$ & & 9.250 & NR2 & Dirkes-G & SAC/S\&T FY00 data comp & 2000 \\
\hline & & & & & $1325-N$ springs & Search-89, Table 5 & 1989 \\
\hline & & & & & Seep spot 4 & UNI-3866, Fig. 1 (report) & 1986 \\
\hline & & & & & $9-4$ & PNL-5289 (report) & 1984 \\
\hline \multirow[t]{3}{*}{ S1057 } & Dirkes-H & & 9.275 & NR2 & Dirkes-H & SAC/S\&T FY00 data comp & 2000 \\
\hline & & & & & 094-1 & WHC-0609, Table A-1 & 1992 \\
\hline & & & & & 094-1 & DOE/RL-92-12, Appendix F & 1992 \\
\hline \multirow[t]{14}{*}{ S1058 } & SN-094-1 & SN-0931 & 9.310 & NR2 & "A" & SESP field survey, 3/30/04 & 2004 \\
\hline & & & & & SN-NS8-13 & PNNL-13404 (gw report) & 2002 \\
\hline & & & & & 100-N Spring 8-13 & PNNL-13418 (env schedule) & 2001 \\
\hline & & & & & 100-n spring 8-13 & PNNL-13230 (env report) & 2000 \\
\hline & & & & & 100-N Spring 8-13 & PNNL-13109 (env schedule) & 2000 \\
\hline & & & & & 100n8.13mileapprox9.2 & PNNL-12088 (env report) & 2000 \\
\hline & & & & & 100n8.13mileapprox9.2 & PNNL-98/99 GPS survey & 1999 \\
\hline & & & & & 100-N Spring 8-13 & PNNL-12103 (env schedule) & 1999 \\
\hline & & & & & 100n8.13mileapprox9.2 & SESP-SLM-01, S-2.4 & 1999 \\
\hline & & & & & 100-N Spring 8-13 & PNNL-11803 (env schedule) & 1998 \\
\hline & & & & & 100-N Spring & PNNL-11464 (env schedule) & 1997 \\
\hline & & & & & 100-N Spring 8-13 & PNNL-10950 (env schedule) & 1996 \\
\hline & & & & & 100 N Spring & PNL-1995 GPS survey & 1995 \\
\hline & & & & & 100-N Spring 8-13 & PNL-10423 (env schedule) & 1995 \\
\hline S1059 & SN-NS8-13 & SN-0933 & 9.325 & NR2 & "B" & SESP field survey, 3/30/04 & 2004 \\
\hline \multirow[t]{2}{*}{ S1060 } & Dirkes-I & SN-0936 & 9.360 & NR2 & "C" & SESP field survey, 3/30/04 & 2004 \\
\hline & & & & & Dirkes-I & SAC/S\&T FY00 data comp & 2000 \\
\hline S1061 & Dirkes-J & & 9.410 & NR2 & Dirkes-J & SAC/S\&T FYO0 data comp & 2000 \\
\hline S1062 & Dirkes-K & & 9.490 & NR2 & Dirkes-K & SAC/S\&T FY00 data comp & 2000 \\
\hline S1063 & $095-1$ & & 9.500 & NR2 & $095-1$ & WHC-0609, Table A-1 & 1992 \\
\hline S1064 & Dirkes-M & & 9.570 & NR2 & Dirkes-M & SAC/S\&T FY00 data comp & 2000 \\
\hline S1065 & Dirkes-N & & 9.625 & NR2 & Dirkes-N & SAC/S\&T FY00 data comp & 2000 \\
\hline \multirow[t]{10}{*}{ S1066 } & SD-098-1 & & 9.700 & NR2 & 100d9.8seep & PNNL-98/99 GPS survey & 1999 \\
\hline & & & & & SD-098-1 & PNNL-13404 (gw report) & 2002 \\
\hline & & & & & 100d9.8seep & PNNL-12088 (env report) & 2000 \\
\hline & & & & & 100d9.8seep & SESP-SLM-01, S-2.4 & 1999 \\
\hline & & & & & SD-098-1 & HR3D-RI 1998 (SAF C99-005) & 1998 \\
\hline & & & & & SD-098-1 & HR3D-RI 1997 (SAF C98-003) & 1997 \\
\hline & & & & & SD-098-1 & HR3D-RI 1996 (CCN 041996) & 1996 \\
\hline & & & & & $098-1$ & HR3D-RI NPL No. 107(D) & 1996 \\
\hline & & & & & 098-1 & WHC-0609, Table A-1 & 1992 \\
\hline & & & & & Below100N & PNL-7500 (report) & 1990 \\
\hline S1067 & Dirkes-O & SN-0986 & 9.860 & NR2 & Dirkes-O & SAC/S\&T FY00 data comp & 2000 \\
\hline S1068 & Dirkes-L & & 9.930 & NR2 & Dirkes-L & SAC/S\&T FY00 data comp & 2000 \\
\hline S1069 & Dirkes-P & SN-0995 & 9.950 & NR2 & Dirkes-P & SAC/S\&T FY00 data comp & 2000 \\
\hline S1070 & Dirkes-Q & & 10.030 & HR3D & Dirkes-Q & SAC/S\&T FY00 data comp & 2000 \\
\hline \multirow[t]{2}{*}{ S1071 } & SD-102-1 & SD-1024 & 10.240 & HR3D & 100d10.2crhot & PNNL-98/99 GPS survey & 1999 \\
\hline & & & & & SD-102-1 & PNNL-13404 (gw report) & 2002 \\
\hline
\end{tabular}


Table 4.2. (contd)

\begin{tabular}{|c|c|c|c|c|c|c|c|}
\hline $\begin{array}{c}\text { Site } \\
\text { Identifier } \\
\text { (proposed) }\end{array}$ & $\begin{array}{l}\text { Spring Name } \\
\text { (use in 2009) }\end{array}$ & $\begin{array}{c}\text { Spring } \\
\text { Name } \\
\text { (proposed) }\end{array}$ & $\begin{array}{c}\text { HRM } \\
\text { ("2k") }\end{array}$ & Segment $^{1}$ & $\begin{array}{c}\text { Name as Used in } \\
\text { Reference }\end{array}$ & Name Reference & $\begin{array}{c}\text { Year of } \\
\text { Name } \\
\text { Use }^{2}\end{array}$ \\
\hline & & & & & 100-D Spring 102-1 & PNNL-13418 (env schedule) & 2001 \\
\hline & & & & & 100-d spring 102-1 & PNNL-13230 (env report) & 2000 \\
\hline & & & & & 100-D Spring 102-1 & PNNL-13109 (env schedule) & 2000 \\
\hline & & & & & $100 \mathrm{~d} 10.2 \mathrm{crhot}$ & PNNL-12088 (env report) & 2000 \\
\hline & & & & & 100-D Spring 102-1 & PNNL-12103 (env schedule) & 1999 \\
\hline & & & & & $100 \mathrm{~d} 10.2 \mathrm{crhot}$ & SESP-SLM-01, S-2.4 & 1999 \\
\hline & & & & & SD-102-1 & HR3D-RI 1998 (SAF C99-005) & 1998 \\
\hline & & & & & SD-102-1 & HR3D-RI 1997 (SAF C98-003) & 1997 \\
\hline & & & & & SD-102-1 & HR3D-RI 1996 (CCN 041996) & 1996 \\
\hline & & & & & $102-1$ & HR3D-RI NPL No. 107(D) & 1996 \\
\hline \multirow[t]{2}{*}{ S1072 } & SD-105-1 & SD-1050 & 10.500 & HR3D & SD-105-1 & HGIS GPS-ID 3020 & 1996 \\
\hline & & & & & SD-104-1 & HEIS & 1995 \\
\hline S1073 & SD-107-1 & SD-1068 & 10.680 & HR3D & SD-107-1 & HGIS GPS-ID 3014 & 1996 \\
\hline \multirow[t]{3}{*}{ S1074 } & SD-108-1 & SD-1081 & 10.810 & HR3D & SD-108-1 & HGIS GPS-ID 3008 & 1996 \\
\hline & & & & & $108-1$ & WHC-0609, Table A-1 & 1992 \\
\hline & & & & & $\mathrm{d} / \mathrm{s}$ of $\mathrm{D}$ & Search-89, Table 5 & 1989 \\
\hline \multirow[t]{2}{*}{ S1075 } & SD-109-1 & SD-1088 & 10.875 & HR3D & SD-109-1 & HGIS GPS-ID 3005 & 1996 \\
\hline & & & & & SD-109-1b & HGIS GPS-ID 3006 & 1996 \\
\hline \multirow[t]{3}{*}{ S1076 } & $109-1 \mathrm{c}$ & & 10.925 & HR3D & SD-109-1c & HGIS GPS-ID 3028 & 1996 \\
\hline & & & & & $110-1$ & WHC-0609, Table A-1 & 1992 \\
\hline & & & & & $110-1$ & DOE/RL-92-12, Appendix F & 1992 \\
\hline \multirow[t]{24}{*}{ S1077 } & SD-110-1 & SD-1103 & 11.025 & HR3D & 100d11.02seep & PNNL-98/99 GPS survey & 1999 \\
\hline & & & & & SD-110-1 & PNNL-13404 (gw report) & 2002 \\
\hline & & & & & 100-D Spring 110-1 & PNNL-13418 (env schedule) & 2001 \\
\hline & & & & & $100-d$ spring $110-1$ & PNNL-13230 (env report) & 2000 \\
\hline & & & & & 100-D Spring 110-1 & PNNL-13109 (env schedule) & 2000 \\
\hline & & & & & 100d11.02seep & PNNL-12088 (env report) & 2000 \\
\hline & & & & & 100-D Spring 110-1 & PNNL-12103 (env schedule) & 1999 \\
\hline & & & & & 100d11.02seep & SESP-SLM-01, S-2.4 & 1999 \\
\hline & & & & & SD-110-1 & HR3D-RI 1998 (SAF C99-005) & 1998 \\
\hline & & & & & 100-D Spring & PNNL-11803 (env schedule) & 1998 \\
\hline & & & & & SD-110-1 & HR3D-RI 1997 (SAF C98-003) & 1997 \\
\hline & & & & & 100-D Spring & PNNL-11464 (env schedule) & 1997 \\
\hline & & & & & SD-110-1 & HGIS GPS-ID 3030 & 1996 \\
\hline & & & & & SD-110-1 & HR3D-RI 1996 (CCN 041996) & 1996 \\
\hline & & & & & $110-1$ & HR3D-RI NPL No. 107(D) & 1996 \\
\hline & & & & & 100-D Spring & PNNL-10950 (env schedule) & 1996 \\
\hline & & & & & $100 \mathrm{D}$ Spring & PNL-1995 GPS survey & 1995 \\
\hline & & & & & 100 D Spring & PNL-1995 GPS survey & 1995 \\
\hline & & & & & 100-D Spring & PNL-10423 (env schedule) & 1995 \\
\hline & & & & & $110-1$ & HR3D-RI 1993 & 1993 \\
\hline & & & & & $110-2$ & WHC-0609, Table A-1 & 1992 \\
\hline & & & & & $110-2$ & DOE/RL-92-12, Appendix F & 1992 \\
\hline & & & & & $11-1$ & PNL-7500 (report) & 1990 \\
\hline & & & & & $11-1$ & PNL-5289 (report) & 1984 \\
\hline \multirow[t]{7}{*}{ S1078 } & SD-110-2 & SD-1112 & 11.115 & HR3D & SD-110-2 & HGIS GPS-ID 3031 & 1996 \\
\hline & & & & & SD-110-2 & PNNL-13404 (gw report) & 2002 \\
\hline & & & & & SD-110-2 & HR3D-RI (SAF C98-003) & 1997 \\
\hline & & & & & SD-110-3 & HGIS GPS-ID 3032 & 1996 \\
\hline & & & & & SD-110-2 & HR3D-RI (CCN 041996) & 1996 \\
\hline & & & & & $110-2$ & HR3D-RI NPL No. 107(D) & 1996 \\
\hline & & & & & $111-1$ & WHC-0609, Table A-1 & 1992 \\
\hline
\end{tabular}


Table 4.2. (contd)

\begin{tabular}{|c|c|c|c|c|c|c|c|}
\hline $\begin{array}{c}\text { Site } \\
\text { Identifier } \\
\text { (proposed) }\end{array}$ & $\begin{array}{l}\text { Spring Name } \\
\text { (use in 2009) }\end{array}$ & $\begin{array}{c}\text { Spring } \\
\text { Name } \\
\text { (proposed) }\end{array}$ & $\begin{array}{c}\text { HRM } \\
(" 2 k ")\end{array}$ & Segment $^{1}$ & $\begin{array}{c}\text { Name as Used in } \\
\text { Reference }\end{array}$ & Name Reference & $\begin{array}{c}\text { Year of } \\
\text { Name } \\
\text { Use }^{2}\end{array}$ \\
\hline & & & & & large, $d / s$ of $D$ & Search-89, Table 5 & 1989 \\
\hline \multirow[t]{9}{*}{ S1079 } & SH-144-1 & SH-1422 & 14.220 & $\mathrm{HR} 3 \mathrm{H}$ & $100 h 144$ & PNNL-98/99 GPS survey & 1999 \\
\hline & & & & & $\mathrm{SH}-144-1$ & PNNL-13404 (gw report) & 2002 \\
\hline & & & & & 100h144 & PNNL-12088 (env report) & 2000 \\
\hline & & & & & $100 h 144$ & SESP-SLM-01, S-2.4 & 1999 \\
\hline & & & & & SH-144-1 & HR3H-RI 1998 (SAF C99-005) & 1998 \\
\hline & & & & & SH-144-1 & HR3H-RI 1997 (SAF C98-003) & 1997 \\
\hline & & & & & SH-144-1 & HGIS GPS-ID 2040 & 1996 \\
\hline & & & & & SH-144-1 & HR3H-RI 1996 (CCN 041996) & 1996 \\
\hline & & & & & $144-1$ & HR3H-RI NPL No. 107(H) & 1996 \\
\hline \multirow[t]{4}{*}{$\mathrm{S} 1080$} & Dirkes-R & & 14.280 & $\mathrm{HR} 3 \mathrm{H}$ & Dirkes-R & SAC/S\&T FY00 data comp & 2000 \\
\hline & & & & & $144-1$ & WHC-0609, Table A-1 & 1992 \\
\hline & & & & & $143-1$ & DOE/RL-92-12, Appendix F & 1992 \\
\hline & & & & & $144-1$ & DOE/RL-92-12, Appendix F & 1992 \\
\hline \multirow[t]{12}{*}{ S1081 } & SH-145-1 & SH-1438 & 14.380 & $\mathrm{HR} 3 \mathrm{H}$ & 100h145seepq & PNNL-98/99 GPS survey & 1999 \\
\hline & & & & & SH-145-1 & PNNL-13404 (gw report) & 2002 \\
\hline & & & & & 100-H Spring 145-1 & PNNL-13418 (env schedule) & 2001 \\
\hline & & & & & 100-h spring 145-1 & PNNL-13230 (env report) & 2000 \\
\hline & & & & & 100-H Spring 145-1 & PNNL-13109 (env schedule) & 2000 \\
\hline & & & & & 100h145seepq & PNNL-12088 (env report) & 2000 \\
\hline & & & & & 100h145seepq & SESP-SLM-01, S-2.4 & 1999 \\
\hline & & & & & $145-1$ & HR3H-RI 1998 (SAF C99-005) & 1998 \\
\hline & & & & & SH-145-1 & HR3H-RI 1997 (SAF C98-003) & 1997 \\
\hline & & & & & SH-145-1 & HGIS GPS-ID 2038 & 1996 \\
\hline & & & & & $145-1$ & HR3H-RI NPL No. 107(H) & 1996 \\
\hline & & & & & $14-4$ & PNL-5289 (report) & 1984 \\
\hline \multirow[t]{8}{*}{ S1082 } & SH-145-2 & SH-1444 & 14.440 & $\mathrm{HR} 3 \mathrm{H}$ & $\mathrm{SH}-145-2$ & HGIS GPS-ID 2037 & 1996 \\
\hline & & & & & 100-H Spring 145-2 & PNNL-12103 (env schedule) & 1999 \\
\hline & & & & & SH-145-2 & HR3H-RI (CCN 041996) & 1996 \\
\hline & & & & & $145-2$ & HR3H-RI NPL No. 107(H) & 1996 \\
\hline & & & & & $145-2$ & HR3H-RI 1993 & 1993 \\
\hline & & & & & $145-1$ & WHC-0609, Table A-1 & 1992 \\
\hline & & & & & $144-1$ & DOE/RL-92-12, Appendix F & 1992 \\
\hline & & & & & $145-1$ & DOE/RL-92-12, Appendix F & 1992 \\
\hline \multirow[t]{4}{*}{ S1083 } & Dirkes-S & & 14.525 & $\mathrm{HR} 3 \mathrm{H}$ & Dirkes-S & SAC/S\&T FY00 data comp & 2000 \\
\hline & & & & & $145-2$ & WHC-0609, Table A-1 & 1992 \\
\hline & & & & & $146-1$ & DOE/RL-92-12, Appendix F & 1992 \\
\hline & & & & & $145-2$ & DOE/RL-92-12, Appendix F & 1992 \\
\hline \multirow[t]{2}{*}{ S1084 } & $14-5$ & & 14.560 & $\mathrm{HR} 3 \mathrm{H}$ & $145-3$ & WHC-0609, Table A-1 & 1992 \\
\hline & & & & & $14-5$ & PNL-5289 (report) & 1984 \\
\hline \multirow[t]{2}{*}{ S1085 } & $147-1$ & & 14.760 & $\mathrm{HR} 3 \mathrm{H}$ & $147-1$ & WHC-0609, Table A-1 & 1992 \\
\hline & & & & & u/s of H@pt. & Search-89, Table 5 & 1989 \\
\hline \multirow[t]{10}{*}{ S1086 } & SH-150-1 & SH-1502 & 15.020 & $\mathrm{HR} 3 \mathrm{H}$ & $100 \mathrm{~h} 150$ & PNNL-98/99 GPS survey & 1999 \\
\hline & & & & & SH-150-1 & PNNL-13404 (gw report) & 2002 \\
\hline & & & & & $100 \mathrm{~h} 150$ & PNNL-12088 (env report) & 2000 \\
\hline & & & & & 100h150 & SESP-SLM-01, S-2.4 & 1999 \\
\hline & & & & & SH-150-1 & HR3H-RI 1998 (SAF C99-005) & 1998 \\
\hline & & & & & SH-150-1 & HR3H-RI 1997 (SAF C98-003) & 1997 \\
\hline & & & & & $\mathrm{SH}-150-2$ & HGIS GPS-ID 2023 & 1996 \\
\hline & & & & & SH-150-1 & HGIS GPS-ID 2024 & 1996 \\
\hline & & & & & SH-150-3 & HR3H-RI 1996 (CCN 041996) & 1996 \\
\hline & & & & & $150-1$ & HR3H-RI NPL No. 107(H) & 1996 \\
\hline
\end{tabular}


Table 4.2. (contd)

\begin{tabular}{|c|c|c|c|c|c|c|c|}
\hline $\begin{array}{c}\text { Site } \\
\text { Identifier } \\
\text { (proposed) }\end{array}$ & $\begin{array}{l}\text { Spring Name } \\
\text { (use in 2009) }\end{array}$ & $\begin{array}{c}\text { Spring } \\
\text { Name } \\
\text { (proposed) }\end{array}$ & $\begin{array}{c}\text { HRM } \\
\text { ("2k") }\end{array}$ & Segment $^{1}$ & $\begin{array}{c}\text { Name as Used in } \\
\text { Reference }\end{array}$ & Name Reference & $\begin{array}{c}\text { Year of } \\
\text { Name } \\
\text { Use }\end{array}$ \\
\hline & & & & & $150-1$ & WHC-0609, Table A-1 & 1992 \\
\hline & & & & & $149-1$ & DOE/RL-92-12, Appendix F & 1992 \\
\hline & & & & & $150-1$ & DOE/RL-92-12, Appendix F & 1992 \\
\hline & & & & & $15-0$ & PNL-7500 (report) & 1990 \\
\hline & & & & & $15-0$ & PNL-5289 (report) & 1984 \\
\hline \multirow[t]{2}{*}{ S1087 } & $152-1$ & & 15.060 & HR3H & SH-152-1 (150-3?) & HGIS GPS-ID 2022 & 1996 \\
\hline & & & & & $152-1$ & WHC-0609, Table A-1 & 1992 \\
\hline S1088 & 151 & & 15.115 & HR3H & SH-152-2 (151-1?) & HGIS GPS-ID 2018 & 1996 \\
\hline \multirow[t]{24}{*}{ S1089 } & $\mathrm{SH}-152-2$ & SH-1526 & 15.265 & $\mathrm{HR} 3 \mathrm{H}$ & 100h152seep & PNNL-98/99 GPS survey & 1999 \\
\hline & & & & & $\mathrm{SH}-152-2$ & PNNL-13404 (gw report) & 2002 \\
\hline & & & & & 100-H Spring 152-2 & PNNL-13418 (env schedule) & 2001 \\
\hline & & & & & 100-H Spring 152-2 & PNNL-13109 (env schedule) & 2000 \\
\hline & & & & & 100h152seep & PNNL-12088 (env report) & 2000 \\
\hline & & & & & 100-H Spring 152-2 & PNNL-12103 (env schedule) & 1999 \\
\hline & & & & & 100h152seep & SESP-SLM-01, S-2.4 & 1999 \\
\hline & & & & & $\mathrm{SH}-152-2$ & HR3H-RI 1998 (SAF C99-005) & 1998 \\
\hline & & & & & 100-H Spring & PNNL-11803 (env schedule) & 1998 \\
\hline & & & & & SH-152-2 & HR3H-RI 1997 (SAF C98-003) & 1997 \\
\hline & & & & & 100-H Spring & PNNL-11464 (env schedule) & 1997 \\
\hline & & & & & SH-152-2 & HR3H-RI 1996 (CCN 041996) & 1996 \\
\hline & & & & & $152-2$ & HR3H-RI NPL No. 107(H) & 1996 \\
\hline & & & & & $152-3$ & HR3H-RI NPL No. 107(H) & 1996 \\
\hline & & & & & 100-H Spring & PNNL-10950 (env schedule) & 1996 \\
\hline & & & & & $100 \mathrm{H}$ Spring & PNL-1995 GPS survey & 1995 \\
\hline & & & & & $100 \mathrm{H}$ Spring & PNL-1995 GPS survey & 1995 \\
\hline & & & & & 100-H Spring & PNL-10423 (env schedule) & 1995 \\
\hline & & & & & $152-2$ & HR3H-RI 1993 & 1993 \\
\hline & & & & & $152-3$ & HR3H-RI 1993 & 1993 \\
\hline & & & & & $152-2$ & WHC-0609, Table A-1 & 1992 \\
\hline & & & & & $152-3$ & WHC-0609, Table A-1 & 1992 \\
\hline & & & & & $\mathrm{H}$ curtain wall & Search-89, Table 5 & 1989 \\
\hline & & & & & H outfall pipe & Search-89, Table 5 & 1989 \\
\hline \multirow[t]{3}{*}{$\mathrm{S} 1090$} & SH-152-4 & SH-1532 & 15.315 & HR3H & SH-152-4 & HGIS GPS-ID 2008 & 1996 \\
\hline & & & & & SH-152-4 & PNNL-13404 (gw report) & 2002 \\
\hline & & & & & $15-4$ & PNL-5289 (report) & 1984 \\
\hline \multirow[t]{15}{*}{ S1091 } & SH-153-1 & SH-1534 & 15.340 & $\mathrm{HR} 3 \mathrm{H}$ & 100h153seep & PNNL-98/99 GPS survey & 1999 \\
\hline & & & & & SH-153-1 & PNNL-13404 (gw report) & 2002 \\
\hline & & & & & 100-h spring 153-1 & PNNL-13230 (env report) & 2000 \\
\hline & & & & & 100h153seep & PNNL-12088 (env report) & 2000 \\
\hline & & & & & 100h153seep & SESP-SLM-01, S-2.4 & 1999 \\
\hline & & & & & SH-153-1 & HR3H-RI 1998 (SAF C99-005) & 1998 \\
\hline & & & & & SH-153-1 & HR3H-RI 1997 (SAF C98-003) & 1997 \\
\hline & & & & & SH-153-1 & HGIS GPS-ID 2006 & 1996 \\
\hline & & & & & SH-153-1 & HR3H-RI 1996 (CCN 041996) & 1996 \\
\hline & & & & & $153-1$ & HR3H-RI NPL No. 107(H) & 1996 \\
\hline & & & & & $153-1$ & HR3H-RI 1993 & 1993 \\
\hline & & & & & 153-1 & WHC-0609, Table A-1 & 1992 \\
\hline & & & & & $154-1$ & DOE/RL-92-12, Appendix F & 1992 \\
\hline & & & & & $153-1$ & DOE/RL-92-12, Appendix F & 1992 \\
\hline & & & & & $15-5$ & PNL-5289 (report) & 1984 \\
\hline \multirow[t]{2}{*}{$\mathrm{S} 1092$} & $158-1$ & & 15.600 & $\mathrm{HR} 3 \mathrm{H}$ & $158-1$ & WHC-0609, Table A-1 & 1992 \\
\hline & & & & & $\mathrm{d} / \mathrm{s}$ of $\mathrm{H}$ & Search-89, Table 5 & 1989 \\
\hline
\end{tabular}


Table 4.2. (contd)

\begin{tabular}{|c|c|c|c|c|c|c|c|}
\hline $\begin{array}{c}\text { Site } \\
\text { Identifier } \\
\text { (proposed) }\end{array}$ & $\begin{array}{l}\text { Spring Name } \\
\text { (use in 2009) }\end{array}$ & $\begin{array}{c}\text { Spring } \\
\text { Name } \\
\text { (proposed) }\end{array}$ & $\begin{array}{l}\text { HRM } \\
\text { ("2k") }\end{array}$ & Segment $^{1}$ & $\begin{array}{l}\text { Name as Used in } \\
\text { Reference }\end{array}$ & Name Reference & $\begin{array}{l}\text { Year of } \\
\text { Name } \\
\text { Use }\end{array}$ \\
\hline \multirow[t]{3}{*}{ S1093 } & $178-1$ & & 17.400 & $\mathrm{HR} 3 \mathrm{H}$ & $178-1$ & unknown & 1991 \\
\hline & & & & & $178-1$ & WHC-0609, Table A-1 & 1992 \\
\hline & & & & & EWB Slough & Search-89, Table 5 & 1989 \\
\hline \multirow[t]{3}{*}{ S1094 } & $18-0$ & & 17.975 & FR3 & $180-1$ & WHC-0609, Table A-1 & 1992 \\
\hline & & & & & pipe $d / s$ EWB & Search-89, Table 5 & 1989 \\
\hline & & & & & $18-0$ & PNL-5289 (report) & 1984 \\
\hline \multirow[t]{2}{*}{ S1095 } & $18-1$ & & 18.110 & FR3 & $180-2$ & WHC-0609, Table A-1 & 1992 \\
\hline & & & & & $18-1$ & PNL-5289 (report) & 1984 \\
\hline \multirow[t]{3}{*}{ S1096 } & $18-2$ & & 18.620 & FR3 & $185-1$ & WHC-0609, Table A-1 & 1992 \\
\hline & & & & & $186-1$ & WHC-0609, Table A-1 & 1992 \\
\hline & & & & & $18-2$ & PNL-5289 (report) & 1984 \\
\hline \multirow[t]{8}{*}{ S1097 } & SF-187-1 & SF-1880 & 18.800 & FR3 & $100 f g w 187$ & PNNL-98/99 GPS survey & 1999 \\
\hline & & & & & SF-187-1 & PNNL-13404 (gw report) & 2002 \\
\hline & & & & & $100 f g w 187$ & PNNL-12088 (env report) & 2000 \\
\hline & & & & & $187-1$ & FR3-RI NPL No. M-15-99-02 & 1999 \\
\hline & & & & & 100fgw187 & SESP-SLM-01, S-2.4 & 1999 \\
\hline & & & & & $187-1$ & FR3-RI NPL No. M-15-96-06 & 1996 \\
\hline & & & & & $187-1$ & WHC-0609, Table A-1 & 1992 \\
\hline & & & & & $187-1$ & DOE/RL-92-12, Appendix F & 1992 \\
\hline \multirow[t]{4}{*}{ S1098 } & 18.3 & & 18.840 & FR3 & 100f18.3seepoutfall & PNNL-98/99 GPS survey & 1999 \\
\hline & & & & & 100f18.3seepoutfall & PNNL-12088 (env report) & 2000 \\
\hline & & & & & 100f18.3seepoutfall & SESP-SLM-01, S-2.4 & 1999 \\
\hline & & & & & 100f18.3seepoutfall & SESP-SLM-01, S-2.4 & 1999 \\
\hline \multirow[t]{2}{*}{ S1099 } & SF-189-1 & SF-1890 & 18.900 & FR3 & $189-1$ & unknown & 1991 \\
\hline & & & & & $189-1$ & FR3-RI 1993 & 1993 \\
\hline \multirow[t]{5}{*}{$\mathrm{S} 1100$} & SF-190-1 & & 18.985 & FR3 & 100fgwseep19 & PNNL-98/99 GPS survey & 1999 \\
\hline & & & & & 100fgwseep19 & PNNL-12088 (env report) & 2000 \\
\hline & & & & & 100fgwseep19 & SESP-SLM-01, S-2.4 & 1999 \\
\hline & & & & & $190-1$ & WHC-0609, Table A-1 & 1992 \\
\hline & & & & & $19-1$ & PNL-5289 (report) & 1984 \\
\hline \multirow[t]{5}{*}{ S1101 } & SF-190-4 & SF-1903 & 19.030 & FR3 & $190-4$ & DOE/RL-92-12, Appendix F & 1992 \\
\hline & & & & & SF-190-4 & PNNL-13404 (gw report) & 2002 \\
\hline & & & & & $190-4$ & FR3-RI NPL No. M-15-99-02 & 1999 \\
\hline & & & & & $190-4$ & FR3-RI NPL No. M-15-96-06 & 1996 \\
\hline & & & & & $190-4$ & WHC-0609, Table A-1 & 1992 \\
\hline \multirow[t]{2}{*}{ S1102 } & SF-205-1 & SF-1953 & 19.530 & FR3 & $205-1$ & unknown & 1991 \\
\hline & & & & & $205-1$ & FR3-RI 1993 & 1993 \\
\hline \multirow[t]{15}{*}{$\mathrm{S} 1103$} & SF-207-1 & SF-2132 & 21.320 & FR3 & 100fgwseep207 & PNNL-98/99 GPS survey & 1999 \\
\hline & & & & & SF-207-1 & PNNL-13404 (gw report) & 2002 \\
\hline & & & & & 100-F Spring 207-1 & PNNL-13418 (env schedule) & 2001 \\
\hline & & & & & $100-f$ spring $207-1$ & PNNL-13230 (env report) & 2000 \\
\hline & & & & & 100-F Spring 207-1 & PNNL-13109 (env schedule) & 2000 \\
\hline & & & & & 100fgwseep207 & PNNL-12088 (env report) & 2000 \\
\hline & & & & & $207-1$ & FR3-RI NPL No. M-15-99-02 & 1999 \\
\hline & & & & & 100-F Spring 207-1 & PNNL-12103 (env schedule) & 1999 \\
\hline & & & & & 100fgwseep207 & SESP-SLM-01, S-2.4 & 1999 \\
\hline & & & & & SF-207-1 & FR3-RI 1998 (SAF X99-004) & 1998 \\
\hline & & & & & SF-207-1 & FR3-RI 1997 (SAF N98-002) & 1997 \\
\hline & & & & & $207-1$ & PNNL-11472 (env report) & 1997 \\
\hline & & & & & $207-1$ & FR3-RI NPL No. M-15-96-06 & 1996 \\
\hline & & & & & $207-1$ & WHC-0609, Table A-1 & 1992 \\
\hline & & & & & $208-1$ & DOE/RL-92-12, Appendix F & 1992 \\
\hline
\end{tabular}


Table 4.2. (contd)

\begin{tabular}{|c|c|c|c|c|c|c|c|}
\hline $\begin{array}{c}\text { Site } \\
\text { Identifier } \\
\text { (proposed) }\end{array}$ & $\begin{array}{l}\text { Spring Name } \\
\text { (use in 2009) }\end{array}$ & $\begin{array}{c}\text { Spring } \\
\text { Name } \\
\text { (proposed) }\end{array}$ & $\begin{array}{c}\text { HRM } \\
\text { ("2k") }\end{array}$ & Segment $^{1}$ & $\begin{array}{c}\text { Name as Used in } \\
\text { Reference }\end{array}$ & Name Reference & $\begin{array}{c}\text { Year of } \\
\text { Name } \\
\text { Use }^{2}\end{array}$ \\
\hline & & & & & $207-1$ & DOE/RL-92-12, Appendix F & 1992 \\
\hline \multirow[t]{9}{*}{$\mathrm{S} 1104$} & SF-210-1 & SF-2141 & 21.410 & FR3 & 100fsespspring & PNNL-98/99 GPS survey & 1999 \\
\hline & & & & & 100fsespspring & PNNL-12088 (env report) & 2000 \\
\hline & & & & & 100fsespspring & PNNL-98/99 GPS survey & 1999 \\
\hline & & & & & 100fsespspring & SESP-SLM-01, S-2.4 & 1999 \\
\hline & & & & & 100-F Spring & PNNL-11803 (env schedule) & 1998 \\
\hline & & & & & 100-F Spring & PNNL-11464 (env schedule) & 1997 \\
\hline & & & & & 100-F Spring & PNNL-10950 (env schedule) & 1996 \\
\hline & & & & & 100 F Spring & PNL-1995 GPS survey & 1995 \\
\hline & & & & & 100-F Spring & PNL-10423 (env schedule) & 1995 \\
\hline \multirow[t]{3}{*}{ S1105 } & SF-211-1 & SF-2164 & 21.640 & FR3 & $216-1$ & DOE/RL-92-12, Appendix F & 1992 \\
\hline & & & & & $211-1$ & WHC-0609, Table A-1 & 1992 \\
\hline & & & & & 211-1 & DOE/RL-92-12, Appendix F & 1992 \\
\hline \multirow[t]{3}{*}{ S1106 } & SF-213-1 & SF-2190 & 21.900 & FR3 & $218-1$ & DOE/RL-92-12, Appendix F & 1992 \\
\hline & & & & & 213-1 & WHC-0609, Table A-1 & 1992 \\
\hline & & & & & 213-1 & DOE/RL-92-12, Appendix F & 1992 \\
\hline \multirow[t]{6}{*}{ S1107 } & SF-216-1 & SF-2230 & 22.300 & FR3 & $221-1$ & DOE/RL-92-12, Appendix F & 1992 \\
\hline & & & & & $216-1$ & WHC-0609, Table A-1 & 1992 \\
\hline & & & & & $222-2$ & WHC-0609, Table A-1 & 1992 \\
\hline & & & & & $216-1$ & DOE/RL-92-12, Appendix F & 1992 \\
\hline & & & & & $22-1$ & PNL-5289 (report) & 1984 \\
\hline & & & & & $22-1 \mathrm{~A}$ & PNL-5289 (report) & 1984 \\
\hline \multirow[t]{3}{*}{ S1108 } & $22-2$ & & 22.410 & FR3 & $222-1$ & WHC-0609, Table A-1 & 1992 \\
\hline & & & & & $223-1$ & WHC-0609, Table A-1 & 1992 \\
\hline & & & & & $22-2$ & PNL-5289 (report) & 1984 \\
\hline \multirow[t]{2}{*}{$\mathrm{S} 1109$} & $22-3$ & & 22.570 & FR3 & $225-1$ & WHC-0609, Table A-1 & 1992 \\
\hline & & & & & $22-3$ & PNL-5289 (report) & 1984 \\
\hline \multirow[t]{3}{*}{ S1110 } & $22-4$ & & 22.690 & FR3 & $226-1$ & WHC-0609, Table A-1 & 1992 \\
\hline & & & & & F Slough & Search-89, Table 5 & 1989 \\
\hline & & & & & $22-4$ & PNL-5289 (report) & 1984 \\
\hline S1111 & $228-1$ & & 22.860 & FR3 & $228-1$ & WHC-0609, Table A-1 & 1992 \\
\hline \multirow[t]{2}{*}{$\mathrm{S} 1112$} & $23-1$ & & 23.110 & HTS & $230-1$ & WHC-0609, Table A-1 & 1992 \\
\hline & & & & & 23-1 & PNL-5289 (report) & 1984 \\
\hline \multirow[t]{2}{*}{$\mathrm{S} 1113$} & $23-2$ & & 23.375 & HTS & $232-1$ & WHC-0609, Table A-1 & 1992 \\
\hline & & & & & $23-2$ & PNL-5289 (report) & 1984 \\
\hline \multirow[t]{2}{*}{ S1114 } & $23-3$ & & 23.615 & HTS & $235-1$ & WHC-0609, Table A-1 & 1992 \\
\hline & & & & & $23-3$ & PNL-5289 (report) & 1984 \\
\hline \multirow[t]{2}{*}{$\mathrm{S} 1115$} & $23-4 \mathrm{~A}$ & & 23.700 & HTS & $236-1$ & WHC-0609, Table A-1 & 1992 \\
\hline & & & & & $23-4 \mathrm{~A}$ & PNL-5289 (report) & 1984 \\
\hline \multirow[t]{2}{*}{$\mathrm{S} 1116$} & $238-2$ & & 23.800 & HTS & $238-2$ & WHC-0609, Table A-1 & 1992 \\
\hline & & & & & broad cobbles & Search-89, Table 5 & 1989 \\
\hline \multirow[t]{2}{*}{$\mathrm{S} 1117$} & 23-4B & & 23.850 & HTS & $238-1$ & WHC-0609, Table A-1 & 1992 \\
\hline & & & & & 23-4B & PNL-5289 (report) & 1984 \\
\hline \multirow[t]{4}{*}{$\mathrm{S} 1118$} & $241-1$ & ST-2406 & 24.060 & HTS & 241-1 & WHC-0609, Table A-1 & 1992 \\
\hline & & & & & 241-1 & WHC-0609, Table A-1 & 1992 \\
\hline & & & & & $246-1$ & DOE/RL-92-12, Appendix F & 1992 \\
\hline & & & & & $241-1$ & DOE/RL-92-12, Appendix F & 1992 \\
\hline \multirow[t]{4}{*}{ S1119 } & $247-1$ & ST-2472 & 24.720 & HTS & $247-1$ & WHC-0609, Table A-1 & 1992 \\
\hline & & & & & $247-1$ & WHC-0609, Table A-1 & 1992 \\
\hline & & & & & $252-1$ & DOE/RL-92-12, Appendix F & 1992 \\
\hline & & & & & $247-1$ & DOE/RL-92-12, Appendix F & 1992 \\
\hline S1120 & $25-1 S$ & & 24.750 & HTS & $250-1$ & WHC-0609, Table A-1 & 1992 \\
\hline
\end{tabular}


Table 4.2. (contd)

\begin{tabular}{|c|c|c|c|c|c|c|c|}
\hline $\begin{array}{c}\text { Site } \\
\text { Identifier } \\
\text { (proposed) }\end{array}$ & $\begin{array}{l}\text { Spring Name } \\
\text { (use in 2009) }\end{array}$ & $\begin{array}{c}\text { Spring } \\
\text { Name } \\
\text { (proposed) }\end{array}$ & $\begin{array}{l}\text { HRM } \\
\text { ("2k") }\end{array}$ & Segment $^{1}$ & $\begin{array}{l}\text { Name as Used in } \\
\text { Reference }\end{array}$ & Name Reference & $\begin{array}{c}\text { Year of } \\
\text { Name } \\
\text { Use }^{2}\end{array}$ \\
\hline & & & & & $248-1$ & WHC-0609, Table A-1 & 1992 \\
\hline & & & & & Hanford Slough & Search-89, Table 5 & 1989 \\
\hline & & & & & $25-1 S$ & PNL-5289 (report) & 1984 \\
\hline \multirow[t]{5}{*}{ S1121 } & $25-2 S$ & & 24.840 & HTS & $250-2$ & WHC-0609, Table A-1 & 1992 \\
\hline & & & & & $250-3$ & WHC-0609, Table A-1 & 1992 \\
\hline & & & & & $25-2 S$ & PNL-5289 (report) & 1984 \\
\hline & & & & & $25-3 S$ & PNL-5289 (report) & 1984 \\
\hline & & & & & Kashier Spring & USGS \#717 & 1972 \\
\hline \multirow[t]{2}{*}{$\mathrm{S} 1122$} & $25-5 S$ & & 25.220 & HTS & $25-5 S$ & PNL-5289 (report) & 1984 \\
\hline & & & & & $250-5$ & WHC-0609, Table A-1 & 1992 \\
\hline \multirow[t]{2}{*}{ S1123 } & $25-1$ & & 25.240 & HTS & $253-1$ & WHC-0609, Table A-1 & 1992 \\
\hline & & & & & $25-1$ & PNL-5289 (report) & 1984 \\
\hline \multirow[t]{2}{*}{ S1124 } & $25-2$ & & 25.310 & HTS & $255-1$ & WHC-0609, Table A-1 & 1992 \\
\hline & & & & & $25-2$ & PNL-5289 (report) & 1984 \\
\hline \multirow[t]{2}{*}{$\mathrm{S} 1125$} & $25-3$ & & 25.380 & HTS & $255-2$ & WHC-0609, Table A-1 & 1992 \\
\hline & & & & & $25-3$ & PNL-5289 (report) & 1984 \\
\hline \multirow[t]{3}{*}{$\mathrm{S} 1126$} & $25-4$ & & 25.740 & HTS & $25-4$ & PNL-5289 (report) & 1984 \\
\hline & & & & & $25-4$ & PNL-7500 (report) & 1990 \\
\hline & & & & & ferry landing & Search-89, Table 5 & 1989 \\
\hline \multirow[t]{2}{*}{$\mathrm{S} 1127$} & $25-5$ & & 25.760 & HTS & $258-1$ & WHC-0609, Table A-1 & 1992 \\
\hline & & & & & $25-5$ & PNL-5289 (report) & 1984 \\
\hline \multirow[t]{3}{*}{$\mathrm{S} 1128$} & $25-6$ & & 25.780 & HTS & $25-6$ & PNL-5289 (report) & 1984 \\
\hline & & & & & $258-2$ & WHC-0609, Table A-1 & 1992 \\
\hline & & & & & $258-3$ & WHC-0609, Table A-1 & 1992 \\
\hline S1129 & $26-1$ & & 26.200 & HTS & $26-1$ & PNL-5289 (report) & 1984 \\
\hline $\mathrm{S} 1130$ & $26-2$ & & 26.240 & HTS & $26-2$ & PNL-5289 (report) & 1984 \\
\hline S1131 & $26-3$ & & 26.600 & HTS & $26-3$ & PNL-5289 (report) & 1984 \\
\hline S1132 & $26-4$ & & 26.880 & HTS & $26-4$ & PNL-5289 (report) & 1984 \\
\hline \multirow[t]{2}{*}{$\mathrm{S} 1133$} & $27-1$ & & 27.070 & HTS & $27-1$ & PNL-5289 (report) & 1984 \\
\hline & & & & & $27-1$ & PNL-8167 (report) & 1992 \\
\hline \multirow[t]{3}{*}{$\mathrm{S} 1134$} & $27-2$ & & 27.250 & HTS & $27-2$ & PNL-5289 (report) & 1984 \\
\hline & & & & & $27-2$ & PNL-8167 (report) & 1992 \\
\hline & & & & & $27-2$ & PNL-7500 (report) & 1990 \\
\hline \multirow[t]{3}{*}{ S1135 } & $27-3$ & & 27.500 & HTS & $27-3$ & \begin{tabular}{|l|} 
PNL-5289 (report) \\
\end{tabular} & 1984 \\
\hline & & & & & $27-3$ & PNL-8167 (report) & 1992 \\
\hline & & & & & $27-3$ & PNL-7500 (report) & 1990 \\
\hline \multirow[t]{8}{*}{ S1136 } & SHTS-UR28-2 & ST-2779 & 27.790 & HTS & hanftownsiteupriver28 & PNNL-98/99 GPS survey & 1999 \\
\hline & & & & & SHTS-UR28-2 & PNNL-13404 (gw report) & 2002 \\
\hline & & & & & Hanford Spr UR 28-2 & PNNL-13418 (env schedule) & 2001 \\
\hline & & & & & hanford spr ur 28-2 & PNNL-13230 (env report) & 2000 \\
\hline & & & & & Hanford Spr UR 28-2 & PNNL-13109 (env schedule) & 2000 \\
\hline & & & & & hanftownsiteupriver28 & PNNL-12088 (env report) & 2000 \\
\hline & & & & & Hanford Spr UR 28-2 & PNNL-12103 (env schedule) & 1999 \\
\hline & & & & & hanftownsiteupriver28 & SESP-SLM-01, S-2.4 & 1999 \\
\hline \multirow[t]{8}{*}{ S1137 } & SHTS-28-2 & ST-2810 & 28.100 & HTS & hanftownsite28.2 & PNNL-98/99 GPS survey & 1999 \\
\hline & & & & & SHTS-28-2 & PNNL-13404 (gw report) & 2002 \\
\hline & & & & & Hanford Spring 28-2 & PNNL-13418 (env schedule) & 2001 \\
\hline & & & & & hanford spring 28-2 & PNNL-13230 (env report) & 2000 \\
\hline & & & & & Hanford Spring 28-2 & PNNL-13109 (env schedule) & 2000 \\
\hline & & & & & hanftownsite28.2 & PNNL-12088 (env report) & 2000 \\
\hline & & & & & hanftownsite28.2 & PNNL-12088 (env report) & 2000 \\
\hline & & & & & SHTS-28-2 & PNNL-12088 (env report) & 2000 \\
\hline
\end{tabular}


Table 4.2. (contd)

\begin{tabular}{|c|c|c|c|c|c|c|c|}
\hline $\begin{array}{c}\text { Site } \\
\text { Identifier } \\
\text { (proposed) }\end{array}$ & $\begin{array}{l}\text { Spring Name } \\
\text { (use in 2009) }\end{array}$ & $\begin{array}{c}\text { Spring } \\
\text { Name } \\
\text { (proposed) }\end{array}$ & $\begin{array}{c}\text { HRM } \\
\text { ("2k") }\end{array}$ & Segment $^{1}$ & $\begin{array}{c}\text { Name as Used in } \\
\text { Reference }\end{array}$ & Name Reference & $\begin{array}{c}\text { Year of } \\
\text { Name } \\
\text { Use }^{2}\end{array}$ \\
\hline & & & & & Hanford Spring 28-2 & PNNL-12103 (env schedule) & 1999 \\
\hline & & & & & hanftownsite28.2 & SESP-SLM-01, S-2.4 & 1999 \\
\hline & & & & & SHTS-28-2 & PNNL-11795 (env report) & 1998 \\
\hline & & & & & Hanford Spring 28-2 & PNNL-11803 (env schedule) & 1998 \\
\hline & & & & & Hanford Spring 28-2 & PNNL-11464 (env schedule) & 1997 \\
\hline & & & & & Hanford Spring 28-2 & PNNL-10950 (env schedule) & 1996 \\
\hline & & & & & Hanford Spring & PNL-1995 GPS survey & 1995 \\
\hline & & & & & Hanford Spring 28-2 & PNL-10423 (env schedule) & 1995 \\
\hline & & & & & $28-2$ & PNL-8167 (report) & 1992 \\
\hline & & & & & $28-2$ & PNL-7500 (report) & 1990 \\
\hline & & & & & HRM 28 & Search-89, Table 5 & 1989 \\
\hline & & & & & $28-1$ & PNL-5289 (report) & 1984 \\
\hline & & & & & $28-2$ & PNL-5289 (report) & 1984 \\
\hline \multirow[t]{9}{*}{$\mathrm{S} 1138$} & SHTS-DR28-2 & ST-2829 & 28.290 & HTS & hanftsdown28 & PNNL-98/99 GPS survey & 1999 \\
\hline & & & & & SHTS-DR28-2 & PNNL-13404 (gw report) & 2002 \\
\hline & & & & & Hanford Spr DR 28-2 & PNNL-13418 (env schedule) & 2001 \\
\hline & & & & & hanford spr dr 28-2 & PNNL-13230 (env report) & 2000 \\
\hline & & & & & Hanford Spr DR 28-2 & PNNL-13109 (env schedule) & 2000 \\
\hline & & & & & hanftsdown28 & PNNL-12088 (env report) & 2000 \\
\hline & & & & & Hanford Spr DR 28-2 & PNNL-12103 (env schedule) & 1999 \\
\hline & & & & & hanftsdown28 & SESP-SLM-01, S-2.4 & 1999 \\
\hline & & & & & $28-3$ & PNL-5289 (report) & 1984 \\
\hline \multirow[t]{3}{*}{$\mathrm{S} 1139$} & $28-4$ & & 28.660 & HTS & $28-4$ & PNL-5289 (report) & 1984 \\
\hline & & & & & $28-4$ & PNL-8167 (report) & 1992 \\
\hline & & & & & $28-4$ & PNL-7500 (report) & 1990 \\
\hline \multirow[t]{2}{*}{$\mathrm{S} 1140$} & $28-5$ & & 28.875 & HTS & $28-5$ & PNL-5289 (report) & 1984 \\
\hline & & & & & $28-5$ & PNL-8167 (report) & 1992 \\
\hline \multirow[t]{2}{*}{ S1141 } & $29-0$ & & 29.050 & HTS & $29-0$ & PNL-5289 (report) & 1984 \\
\hline & & & & & $29-0$ & PNL-8167 (report) & 1992 \\
\hline \multirow[t]{2}{*}{$\mathrm{S} 1142$} & $25-4 S$ & & 29.090 & HTS & $25-4 S$ & PNL-5289 (report) & 1984 \\
\hline & & & & & $250-4$ & WHC-0609, Table A-1 & 1992 \\
\hline $\mathrm{S} 1143$ & $29-1$ & & 29.240 & $\mathrm{PO} 1$ & $29-1$ & PNL-5289 (report) & 1984 \\
\hline S1144 & $29-2$ & & 29.500 & PO1 & $29-2$ & PNL-5289 (report) & 1984 \\
\hline $\mathrm{S} 1145$ & $29-3$ & & 29.750 & $\mathrm{PO} 1$ & $29-3$ & PNL-5289 (report) & 1984 \\
\hline S1146 & $30-0$ & & 30.040 & PO1 & $30-0$ & PNL-5289 (report) & 1984 \\
\hline \multirow[t]{2}{*}{$\mathrm{S} 1147$} & $30-1$ & & 30.120 & PO1 & $30-1$ & PNL-5289 (report) & 1984 \\
\hline & & & & & $30-1$ & PNL-8167 (report) & 1992 \\
\hline S1148 & $30-2$ & & 30.940 & PO1 & $30-2$ & PNL-5289 (report) & 1984 \\
\hline S1149 & $31-1$ & & 31.000 & PO1 & $31-1$ & PNL-5289 (report) & 1984 \\
\hline $\mathrm{S} 1150$ & $31-2$ & & 31.300 & PO1 & $31-2$ & PNL-5289 (report) & 1984 \\
\hline S1151 & $31-3$ & & 31.495 & PO1 & $31-3$ & PNL-5289 (report) & 1984 \\
\hline S1152 & $31-4$ & & 31.505 & PO1 & $31-4$ & PNL-5289 (report) & 1984 \\
\hline \multirow[t]{2}{*}{$\mathrm{S} 1153$} & $31-5$ & & 31.600 & $\mathrm{PO} 1$ & $31-5$ & PNL-5289 (report) & 1984 \\
\hline & & & & & Opposite Ringold & Search-89, Table 5 & 1989 \\
\hline $\mathrm{S} 1154$ & $32-0$ & & 32.500 & $\mathrm{PO} 1$ & $32-0$ & PNL-5289 (report) & 1984 \\
\hline S1155 & $33-1$ & & 33.000 & PO1 & 33-1 & PNL-5289 (report) & 1984 \\
\hline S1156 & $34-1$ & & 34.000 & PO1 & $34-1$ & PNL-5289 (report) & 1984 \\
\hline S1157 & $34-2$ & & 34.900 & PO1 & $34-2$ & PNL-5289 (report) & 1984 \\
\hline S1158 & $34-3$ & & 34.925 & PO1 & $34-3$ & PNL-5289 (report) & 1984 \\
\hline $\mathrm{S} 1159$ & $34-4$ & & 34.940 & $\mathrm{PO} 1$ & $34-4$ & PNL-5289 (report) & 1984 \\
\hline \multirow[t]{2}{*}{ S1160 } & $35-1$ & & 35.600 & PO1 & $35-1$ & PNL-5289 (report) & 1984 \\
\hline & & & & & WPPSS & Search-89, Table 5 & 1989 \\
\hline
\end{tabular}


Table 4.2. (contd)

\begin{tabular}{|c|c|c|c|c|c|c|c|}
\hline $\begin{array}{c}\text { Site } \\
\text { Identifier } \\
\text { (proposed) }\end{array}$ & $\begin{array}{l}\text { Spring Name } \\
\text { (use in 2009) }\end{array}$ & $\begin{array}{c}\text { Spring } \\
\text { Name } \\
\text { (proposed) }\end{array}$ & $\begin{array}{l}\text { HRM } \\
\text { ("2k") }\end{array}$ & Segment $^{1}$ & $\begin{array}{l}\text { Name as Used in } \\
\text { Reference }\end{array}$ & Name Reference & $\begin{array}{c}\text { Year of } \\
\text { Name } \\
\text { Use }^{2}\end{array}$ \\
\hline \multirow[t]{2}{*}{ S1161 } & $35-2$ & & 36.000 & PO1 & $35-2$ & PNL-5289 (report) & 1984 \\
\hline & & & & & $\mathrm{u} / \mathrm{s}$ of 3 lines & Search-89, Table 5 & 1989 \\
\hline S1162 & $36-1$ & & 36.740 & $\mathrm{PO1}$ & $36-1$ & PNL-5289 (report) & 1984 \\
\hline \multirow[t]{2}{*}{ S1163 } & $37-1$ & & 37.100 & PO1 & $37-1$ & PNL-5289 (report) & 1984 \\
\hline & & & & & Wooded Island Slough & Search-89, Table 5 & 1989 \\
\hline S1164 & $37-2$ & & 37.500 & PO1 & $37-2$ & PNL-5289 (report) & 1984 \\
\hline \multirow[t]{2}{*}{ S1165 } & $38-1$ & & 38.250 & PO1 & $38-1$ & PNL-5289 (report) & 1984 \\
\hline & & & & & $38-1$ & PNL-7500 (report) & 1990 \\
\hline \multirow[t]{2}{*}{ S1166 } & $38-10$ & & 38.850 & PO1 & $38-10$ & PNL-5289 (report) & 1984 \\
\hline & & & & & $38-10$ & PNL-7500 (report) & 1990 \\
\hline S1167 & $39-1$ & & 39.240 & $\mathrm{PO1}$ & $39-1$ & PNL-5289 (report) & 1984 \\
\hline S1168 & $40-1$ & & 40.000 & $\mathrm{PO1}$ & $40-1$ & PNL-5289 (report) & 1984 \\
\hline S1169 & $40-2$ & & 40.020 & $\mathrm{PO} 1$ & $40-2$ & PNL-5289 (report) & 1984 \\
\hline \multirow[t]{2}{*}{$\mathrm{S} 1170$} & Spr-1 & S3-4076 & 40.760 & $3 F F 5$ & Spring 1 & WHC-SD-EN-TI-125, App. A & 1992 \\
\hline & & & & & $40-3$ & PNL-5289 (report) & 1984 \\
\hline \multirow[t]{2}{*}{ S1171 } & Spr-2 & S3-4089 & 40.890 & $3 F F 5$ & Spring 2 & WHC-SD-EN-TI-125, App. A & 1992 \\
\hline & & & & & $40-4$ & PNL-5289 (report) & 1984 \\
\hline S1172 & Spr-3 & S3-4126 & 41.260 & $3 F F 5$ & Spring 3 & WHC-SD-EN-TI-125, App. A & 1992 \\
\hline S1173 & Spr-4 & S3-4132 & 41.325 & 3FF5 & Spring 4 & WHC-SD-EN-TI-125, App. A & 1992 \\
\hline \multirow[t]{3}{*}{ S1174 } & $41-1$ & & 41.500 & $3 F F 5$ & 4 41-1 & PNL-5289 (report) & 1984 \\
\hline & & & & & \begin{tabular}{|l|}
$41-1$ \\
\end{tabular} & PNL-7500 (report) & 1990 \\
\hline & & & & & $u / s$ of 300 & Search-89, Table 5 & 1989 \\
\hline S1175 & Spr-5 & S3-4173 & 41.730 & $3 F F 5$ & Spring 5 & WHC-SD-EN-TI-125, App. A & 1992 \\
\hline \multirow[t]{3}{*}{ S1176 } & Spr-6 & S3-4186 & 41.865 & 3FF5 & Spring 6 & WHC-SD-EN-TI-125, App. A & 1992 \\
\hline & & & & & \begin{tabular}{|l|}
$41-2$ \\
\end{tabular} & PNL-7500 (report) & 1990 \\
\hline & & & & & $41-2$ & PNL-5289 (report) & 1984 \\
\hline \multirow[t]{2}{*}{ S1177 } & $42-1$ & & 42.000 & $3 F F 5$ & $42-1$ & PNL-5289 (report) & 1984 \\
\hline & & & & & $42-1$ & PNL-7500 (report) & 1990 \\
\hline \multirow[t]{18}{*}{$\mathrm{S} 1178$} & S3-42-2 & S3-4211 & 42.105 & 3FF5 & \begin{tabular}{|l|} 
Spring 7 \\
\end{tabular} & WHC-SD-EN-TI-125, App. A & 1992 \\
\hline & & & & & S300-42-2 & PNNL-13404 (gw report) & 2002 \\
\hline & & & & & 300 Area Spring 42-2 & PNNL-13418 (env schedule) & 2001 \\
\hline & & & & & 300 area spring $42-2$ & PNNL-13230 (env report) & 2000 \\
\hline & & & & & 300 Area Spring 42-2 & PNNL-13109 (env schedule) & 2000 \\
\hline & & & & & 300areaseepsespnormal & PNNL-12088 (env report) & 2000 \\
\hline & & & & & 300areaseepsespnormal & PNNL-98/99 GPS survey & 1999 \\
\hline & & & & & 300 Area Spring 42-2 & PNNL-12103 (env schedule) & 1999 \\
\hline & & & & & 300areaseepsespnormal & SESP-SLM-01, S-2.4 & 1999 \\
\hline & & & & & 300 Area Spring 42-2 & PNNL-11803 (env schedule) & 1998 \\
\hline & & & & & 300 Area Spring 42-2 & PNNL-11464 (env schedule) & 1997 \\
\hline & & & & & 300 Area Spring 42-2 & PNNL-10950 (env schedule) & 1996 \\
\hline & & & & & 300 Area Seep & PNL-1995 GPS survey & 1995 \\
\hline & & & & & 300 Area Spring 42-2 & PNL-10423 (env schedule) & 1995 \\
\hline & & & & & $42-2$ & PNL-7500 (report) & 1990 \\
\hline & & & & & 300 Area & Search-89, Table 5 & 1989 \\
\hline & & & & & spring \#42-2 & Search-89, Table 5 & 1989 \\
\hline & & & & & $42-2$ & PNL-5289 (report) & 1984 \\
\hline \multirow[t]{2}{*}{ S1179 } & Spr-8 & S3-4232 & 42.320 & $3 F F 5$ & Spring 8 & WHC-SD-EN-TI-125, App. A & 1992 \\
\hline & & & & & $42-3$ & PNL-5289 (report) & 1984 \\
\hline \multirow[t]{4}{*}{$\mathrm{S} 1180$} & S3-DR42-2 & S3-4236 & 42.365 & $3 F F 5$ & Spring 9 & WHC-SD-EN-TI-125, App. A & 1992 \\
\hline & & & & & S300-DR42-2 & PNNL-13404 (gw report) & 2002 \\
\hline & & & & & 300 Area Spr DR 42-2 & PNNL-13418 (env schedule) & 2001 \\
\hline & & & & & 300 Area Spr DR 42-2 & PNNL-13109 (env schedule) & 2000 \\
\hline
\end{tabular}


Table 4.2. (contd)

\begin{tabular}{|c|c|c|c|c|c|c|c|}
\hline $\begin{array}{c}\text { Site } \\
\text { Identifier } \\
\text { (proposed) }\end{array}$ & $\begin{array}{l}\text { Spring Name } \\
\text { (use in 2009) }\end{array}$ & $\begin{array}{c}\text { Spring } \\
\text { Name } \\
\text { (proposed) }\end{array}$ & $\begin{array}{l}\text { HRM } \\
\text { ("2k") }\end{array}$ & Segment $^{1}$ & $\begin{array}{l}\text { Name as Used in } \\
\text { Reference }\end{array}$ & Name Reference & $\begin{array}{c}\text { Year of } \\
\text { Name } \\
\text { Use }^{2}\end{array}$ \\
\hline & & & & & 300seepdownriver & PNNL-12088 (env report) & 2000 \\
\hline & & & & & 300seepdownriver & PNNL-98/99 GPS survey & 1999 \\
\hline & & & & & 300 Area Spr DR 42-2 & PNNL-12103 (env schedule) & 1999 \\
\hline & & & & & 300seepdownriver & SESP-SLM-01, S-2.4 & 1999 \\
\hline & & & & & $42-4$ & PNL-7500 (report) & 1990 \\
\hline & & & & & $42-4$ & PNL-5289 (report) & 1984 \\
\hline S1181 & Spr-10 & S3-4243 & 42.430 & 3FF5 & Spring 10 & WHC-SD-EN-TI-125, App. A & 1992 \\
\hline \multirow[t]{2}{*}{ S1182 } & Spr-11 & S3-4267 & 42.670 & 3FF5 & Spring 11 & WHC-SD-EN-TI-125, App. A & 1992 \\
\hline & & & & & cascade & Search-89, Table 5 & 1989 \\
\hline \multirow[t]{2}{*}{ S1183 } & Spr-12 & S3-4306 & 43.060 & 3FF5 & Spring 12 & WHC-SD-EN-TI-125, App. A & 1992 \\
\hline & & & & & $u / w d / s$ of 300 & Search-89, Table 5 & 1989 \\
\hline S1184 & Spr-13 & S3-4318 & 43.180 & 3FF5 & Spring 13 & WHC-SD-EN-TI-125, App. A & 1992 \\
\hline \multirow[t]{3}{*}{ S1185 } & Spr-14 & S3-4348 & 43.475 & 3FF5 & Spring 14 & WHC-SD-EN-TI-125, App. A & 1992 \\
\hline & & & & & $43-1$ & PNL-7500 (report) & 1990 \\
\hline & & & & & $43-1$ & PNL-5289 (report) & 1984 \\
\hline S1186 & $43-2$ & & 43.775 & 3FF5 & $43-2$ & PNL-5289 (report) & 1984 \\
\hline \multirow[t]{2}{*}{ S1187 } & $43-3$ & & 43.860 & 3FF5 & $43-3$ & PNL-5289 (report) & 1984 \\
\hline & & & & & $\mathrm{u} / \mathrm{s}$ of Richland inlet & Search-89, Table 5 & 1989 \\
\hline S1188 & SRL-444-1 & & 44.400 & RLD & & & 2004 \\
\hline
\end{tabular}

1 Abbreviations: VB = Vernita Bridge; BC5 = 100-B Area; KR4 = 100-K Area; NR2 = 100-N Area; HR3D = 100-D Area; HR3H = 100-H Area; FR3 = 100-F Area; HTS = Hanford Town Site; PO1 = Hanford town site downstream to 300 Area; $3 F F 5=300$ Area; RLD = North Richland

2 Review of name use in various reports is valid through approximately 2002 . Since then, name use has been reasonably consistent with the names shown under "current use." 



\subsection{Seep Wells in Rip Rap at N-Springs Shoreline}

During the reactor operating years at the 100-N Area (1963 to 1987), a groundwater mound was created by liquid effluent disposal to the 1301-N Crib/Trench. The volume of effluent disposed caused extensive seepage of contaminated groundwater along the riverbank (“N-Springs," Figure 5.1). The seepage contained high levels of radionuclides, which posed a risk to humans and aquatic biota. To mitigate the risk of radiological exposure, large basalt boulders were placed along the shoreline as rip rap in 1984 (Probasco 1986). Fifteen steel casings, referred to as "seep wells," were installed with the rip rap to provide sampling access to the seepage (Figures 5.2 and 5.3). The seep wells have been routinely sampled in the past on an annual basis to satisfy near-field environmental monitoring requirements (Poston et al. 2003).

\section{$5.1 \quad$ N-Springs Seep Well Names and Coordinates}

The names and geographic coordinates for the N-Springs seep wells are listed in Table 5.1. The columns in the table are defined as follows:

- Site Identifier (proposed) - Identifier code proposed for each seep well—for internal data management purposes. This code would be used in the same way as the well identifier code assigned in the HWIS for monitoring wells, boreholes, and aquifer sampling tubes.

- Spring Name - The name appearing in recent reports describing the monitoring results for the nearfield environmental monitoring project.

- Spring Name (alternate) - Alternate name used by other projects for samples collected from NSprings seep well sites.

- HRM (“2k”) - Location relative to the HRM system, as updated in 1999.

- Segment - Segments defined for convenience in grouping monitoring sites by CERCLA groundwater operable units, groundwater interest areas, or other common characteristic.

- Northing and Easting - Location coordinates in Washington State Plane (South Zone) system; units are meters; North American Datum of 1983.

- Coordinate Quality - Quality of the coordinates, e.g., “surveyed” using global position system (GPS) equipment or traditional land surveying methods; "estimated” using geographic information system (GIS) maps.

- Coordinate Type - Equipment or method used to establish coordinates.

- Coordinate Reference - Citation for information on the seep well site.

\subsection{N-Springs Seep Well Name Usage}

The N-Springs seep wells have been sampled for many years, with multiple names being used for individual seep wells. Because the analytical results from sampling these facilities have never been entered into the HEIS database, there has been little pressure to standardize the site names. Table 5.2 provides a listing of names used in many of the previous reports describing use of the seep wells, but no 
claim is made that the list is complete. Many of the columns in Table 5.2 are the same as those used in Table 5.1, with exceptions as follows:

- Name as Used in Reference - Name as used in historical report or other reference that includes NSprings seep well names.

- Name Reference - Citation or other source.

- Year of Name Use - Year in which a seep well name was published in reference cited.

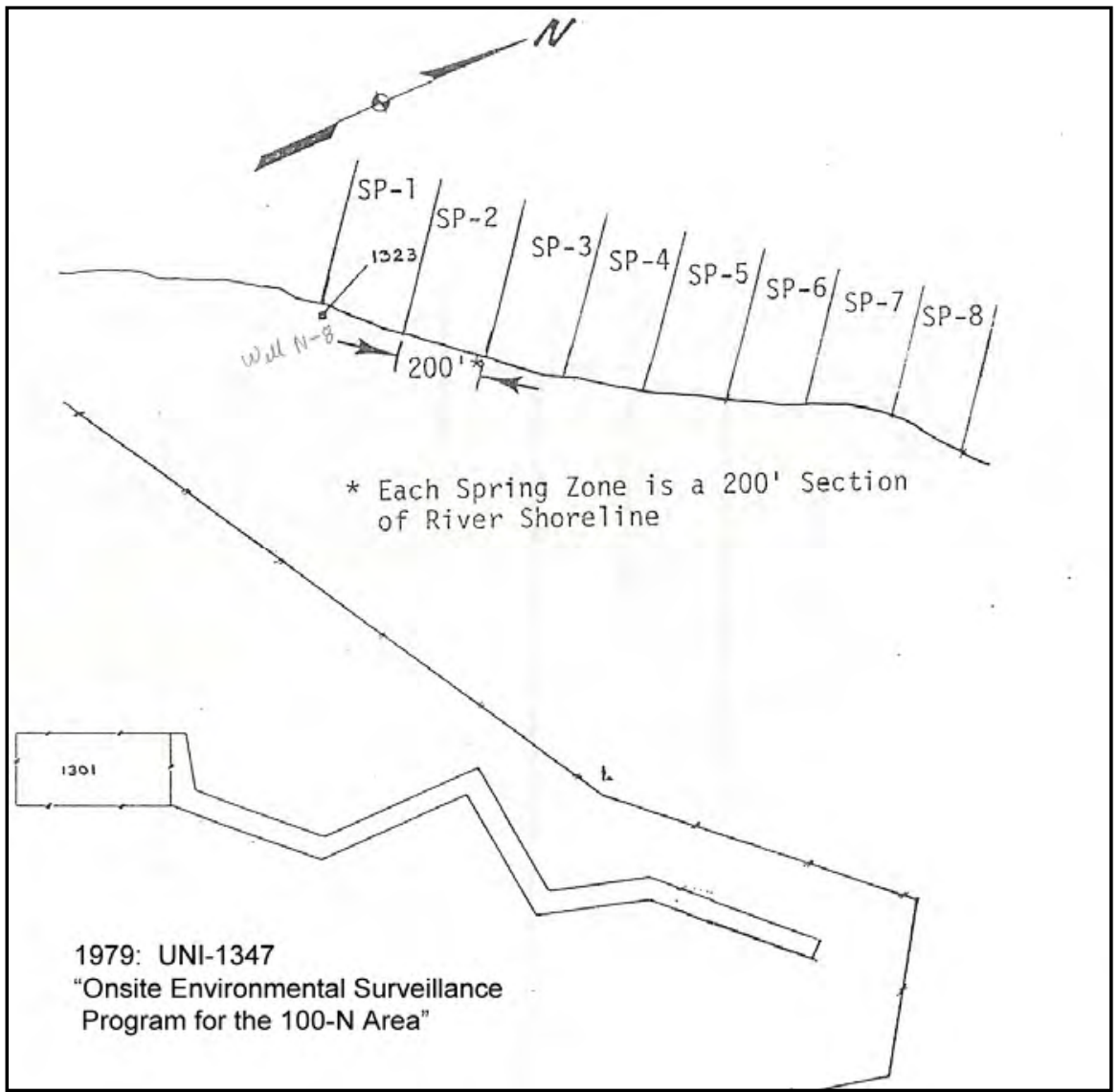

Figure 5.1. Areas of Riverbank Springs at 100-N Area During Operations (Prior to installation of rip rap and seep wells in 1984) 


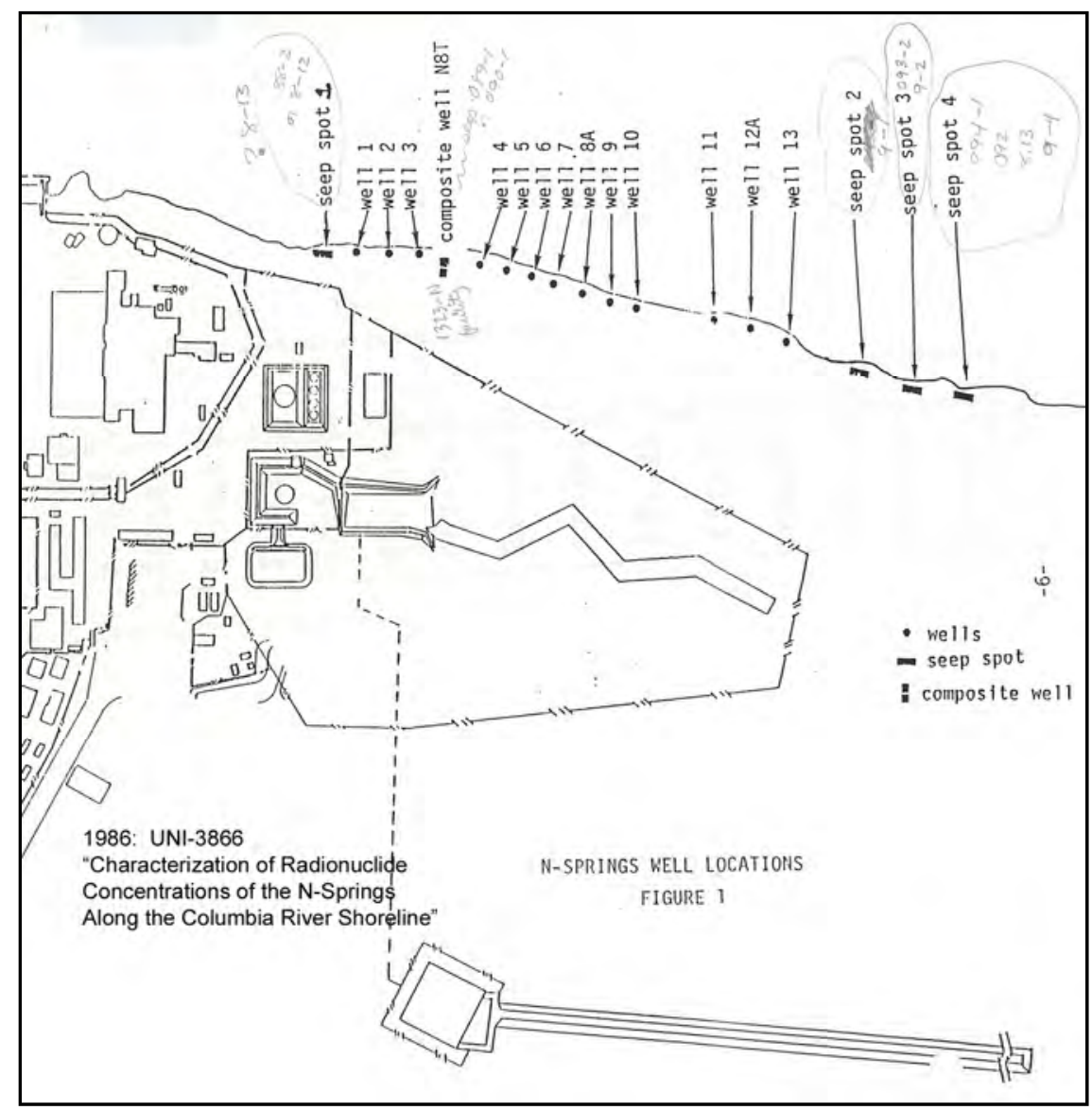

Figure 5.2. Locations of Seep Wells and Seepage Spots Along 100-N Area Shoreline (Post-installation of N-Springs seep wells in 1984)

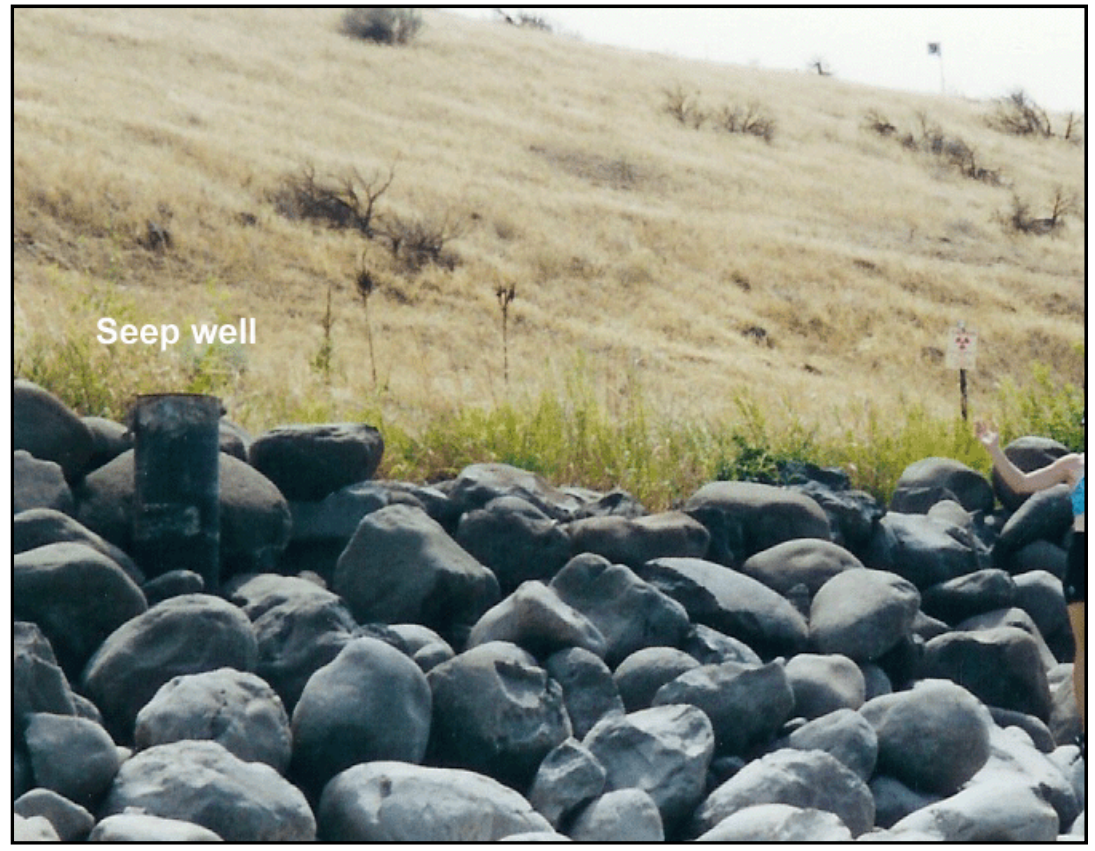

Figure 5.3. Photograph of Seep Well Installed in Rip Rap Along 100-N Area Shoreline 
Table 5.1. Riverbank Seepage Well Casings Along the N-Springs Shoreline, Hanford Site

\begin{tabular}{|c|c|c|c|c|c|c|c|c|c|}
\hline $\begin{array}{c}\text { Site } \\
\text { Identifier } \\
\text { (proposed) }\end{array}$ & $\begin{array}{c}\text { Spring } \\
\text { Name } \\
\text { (use in } \\
\text { 2009) }\end{array}$ & $\begin{array}{l}\text { Spring } \\
\text { Name } \\
\text { (alternate } \\
\text { use) }\end{array}$ & $\begin{array}{l}\text { HRM } \\
\text { ("2k") }\end{array}$ & Segment $^{1}$ & $\begin{array}{c}\text { Northing } \\
\text { (m-NAD83) }\end{array}$ & $\begin{array}{c}\text { Easting } \\
\text { (m-NAD83) }\end{array}$ & $\begin{array}{c}\text { Coordinate } \\
\text { Quality }^{2}\end{array}$ & $\underset{\text { Type }^{2}}{\text { Coordinate }}$ & $\begin{array}{l}\text { Coordinate } \\
\text { Reference }\end{array}$ \\
\hline S2000 & Y301 & NS-1 & 8.880 & NR2 & $149,888.600$ & $571,270.600$ & SURV & GPS & HGIS GPS-ID 5016 \\
\hline S2001 & Y302 & NS-2 & 8.895 & NR2 & $149,911.800$ & $571,290.400$ & SURV & GPS & HGIS GPS-ID 5015 \\
\hline S2002 & Y303 & NS-3 & 8.915 & NR2 & $149,934.300$ & $571,312.500$ & SURV & GPS & HGIS GPS-ID 5014 \\
\hline S2003 & Y304 & NS-4 & 8.930 & NR2 & $149,954.500$ & $571,335.700$ & SURV & GPS & HGIS GPS-ID 5013 \\
\hline S2004 & Y305 & NS-5 & 8.950 & NR2 & $149,978.400$ & $571,351.700$ & SURV & GPS & HGIS GPS-ID 5012 \\
\hline S2005 & Y306 & NS-6 & 8.970 & NR2 & $150,003.500$ & $571,370.200$ & SURV & GPS & HGIS GPS-ID 5011 \\
\hline S2006 & Y307 & NS-7 & 8.985 & NR2 & $150,027.100$ & $571,384.000$ & SURV & GPS & HGIS GPS-ID 5010 \\
\hline S2007 & Y308 & NS-8A & 9.000 & NR2 & $150,051.800$ & $571,396.500$ & SURV & GPS & HGIS GPS-ID 5008 \\
\hline S2008 & NS-8B & NS-8B & 9.001 & NR2 & $150,050.300$ & $571,399.800$ & SURV & GPS & HGIS GPS-ID 5009 \\
\hline S2009 & Y309 & NS-9 & 9.015 & NR2 & $150,080.800$ & $571,412.400$ & SURV & GPS & HGIS GPS-ID 5007 \\
\hline S2010 & Y310 & NS-10 & 9.030 & NR2 & $150,109.500$ & $571,430.500$ & SURV & GPS & HGIS GPS-ID 5006 \\
\hline S2011 & Y311 & NS-11 & 9.100 & NR2 & $150,190.400$ & $571,495.500$ & SURV & GPS & HGIS GPS-ID 5005 \\
\hline S2012 & Y312 & NS-12A & 9.125 & NR2 & $150,216.300$ & $571,513.300$ & SURV & GPS & HGIS GPS-ID 5003 \\
\hline S2013 & NS-12B & NS-12B & 9.126 & NR2 & $150,214.700$ & $571,519.300$ & SURV & GPS & HGIS GPS-ID 5004 \\
\hline S2014 & Y313 & NS-13 & 9.140 & NR2 & $150,251.900$ & $571,542.100$ & SURV & GPS & HGIS GPS-ID 5002 \\
\hline
\end{tabular}


Table 5.2. Historical Names for Riverbank Seepage Well Casings Sites

\begin{tabular}{|c|c|c|c|c|c|c|}
\hline $\begin{array}{c}\text { Site } \\
\text { Identifier } \\
\text { (proposed) }\end{array}$ & $\begin{array}{l}\text { Spring Name } \\
\text { (use in 2009) }\end{array}$ & $\begin{array}{c}\text { Spring Name } \\
\text { (alternate use) }\end{array}$ & $\begin{array}{l}\text { HRM } \\
\text { ("2k") }\end{array}$ & $\begin{array}{c}\text { Name as Used in } \\
\text { Reference }\end{array}$ & Name Reference & $\begin{array}{c}\text { Year of } \\
\text { Name } \\
\text { Use }^{1}\end{array}$ \\
\hline \multirow[t]{16}{*}{ S2000 } & Y301 & NS-1 & 8.880 & Y301 & PNNL-14295, App. 2 & 2003 \\
\hline & & & & Y301 & PNNL-13910, App. 2 & 2002 \\
\hline & & & & C3454 & HEIS2_ADM_WELL & 2001 \\
\hline & & & & $177-\mathrm{N}-1$ & HEIS2_ADM_WELL & 2001 \\
\hline & & & & Y301 & PNNL-13487, App. 2 & 2001 \\
\hline & & & & Y301 & PNNL-13230, App. 2 & 2000 \\
\hline & & & & Y301 & PNNL-12088, App. 2 & 1999 \\
\hline & & & & Y301 & HNF-EP-0573-6 & 1998 \\
\hline & & & & Site 1 & HNF-EP-0573-5 & 1997 \\
\hline & & & & Site 1 & WHC-EP-0573-4 & 1996 \\
\hline & & & & NS-1 & WHC-SP-0098-8, Fig. A-54 & 1996 \\
\hline & & & & NS-1 & BHI-00164 & 1995 \\
\hline & & & & Site 1 & WHC-EP-0573-1 & 1993 \\
\hline & & & & Well 1 & WHC-SP-0480 & 1989 \\
\hline & & & & Well 1 & WHC-SP-0277 & 1988 \\
\hline & & & & Well 1 & UNI-3866 & 1986 \\
\hline \multirow[t]{16}{*}{ S2001 } & Y302 & NS-2 & 8.895 & Y302 & PNNL-14295, App. 2 & 2003 \\
\hline & & & & Y302 & PNNL-13910, App. 2 & 2002 \\
\hline & & & & C3455 & HEIS2_ADM_WELL & 2001 \\
\hline & & & & $177-\mathrm{N}-2$ & HEIS2_ADM_WELL & 2001 \\
\hline & & & & Y302 & PNNL-13487, App. 2 & 2001 \\
\hline & & & & Y302 & PNNL-13230, App. 2 & 2000 \\
\hline & & & & Y302 & PNNL-12088, App. 2 & 1999 \\
\hline & & & & Y302 & HNF-EP-0573-6 & 1998 \\
\hline & & & & Site 2 & HNF-EP-0573-5 & 1997 \\
\hline & & & & Site 2 & WHC-EP-0573-4 & 1996 \\
\hline & & & & NS-2 & WHC-SP-0098-8, Fig. A-54 & 1996 \\
\hline & & & & NS-2 & BHI-00164 & 1995 \\
\hline & & & & Site 2 & WHC-EP-0573-1 & 1993 \\
\hline & & & & Well 2 & WHC-SP-0480 & 1989 \\
\hline & & & & Well 2 & WHC-SP-0277 & 1988 \\
\hline & & & & Well 2 & UNI-3866 & 1986 \\
\hline \multirow[t]{14}{*}{ S2002 } & Y303 & NS-3 & 8.915 & Y303 & PNNL-14295, App. 2 & 2003 \\
\hline & & & & Y303 & PNNL-13910, App. 2 & 2002 \\
\hline & & & & C3456 & HEIS2_ADM_WELL & 2001 \\
\hline & & & & $177-\mathrm{N}-3$ & HEIS2_ADM_WELL & 2001 \\
\hline & & & & Y303 & PNNL-13487, App. 2 & 2001 \\
\hline & & & & Y303 & PNNL-13230, App. 2 & 2000 \\
\hline & & & & Y303 & PNNL-12088, App. 2 & 1999 \\
\hline & & & & Y303 & HNF-EP-0573-6 & 1998 \\
\hline & & & & Site 3 & HNF-EP-0573-5 & 1997 \\
\hline & & & & Site 3 & WHC-EP-0573-4 & 1996 \\
\hline & & & & NS-3 & WHC-SP-0098-8, Fig. A-54 & 1996 \\
\hline & & & & NS-3 & BHI-00164 & 1995 \\
\hline & & & & Site 3 & WHC-EP-0573-1 & 1993 \\
\hline & & & & Well 3 & WHC-SP-0480 & 1989 \\
\hline
\end{tabular}


Table 5.2. (contd)

\begin{tabular}{|c|c|c|c|c|c|c|}
\hline $\begin{array}{c}\text { Site } \\
\text { Identifier } \\
\text { (proposed) }\end{array}$ & $\begin{array}{l}\text { Spring Name } \\
\text { (use in 2009) }\end{array}$ & $\begin{array}{c}\text { Spring Name } \\
\text { (alternate use) }\end{array}$ & $\begin{array}{c}\text { HRM } \\
\text { ("2k") }\end{array}$ & $\begin{array}{c}\text { Name as Used in } \\
\text { Reference }\end{array}$ & Name Reference & $\begin{array}{l}\text { Year of } \\
\text { Name } \\
\text { Use }^{1}\end{array}$ \\
\hline & & & & Well 3 & WHC-SP-0277 & 1988 \\
\hline & & & & Well 3 & UNI-3866 & 1986 \\
\hline \multirow[t]{17}{*}{ S2003 } & Y304 & NS-4 & 8.930 & Y304 & PNNL-14295, App. 2 & 2003 \\
\hline & & & & Y304 & PNNL-13910, App. 2 & 2002 \\
\hline & & & & C3457 & HEIS2_ADM_WELL & 2001 \\
\hline & & & & $177-\mathrm{N}-4$ & HEIS2_ADM_WELL & 2001 \\
\hline & & & & Y304 & PNNL-13487, App. 2 & 2001 \\
\hline & & & & Y304 & PNNL-13230, App. 2 & 2000 \\
\hline & & & & Y304 & PNNL-12088, App. 2 & 1999 \\
\hline & & & & Y304 & HNF-EP-0573-6 & 1998 \\
\hline & & & & Site 4 & HNF-EP-0573-5 & 1997 \\
\hline & & & & Site 4 & WHC-EP-0573-4 & 1996 \\
\hline & & & & NS-4 & WHC-SP-0098-8, Fig. A-54 & 1996 \\
\hline & & & & NS-4 & BHI-00164 & 1995 \\
\hline & & & & Site 4 & WHC-EP-0573-1 & 1993 \\
\hline & & & & $\mathrm{N}$ seep well \#4 & Search 89, Table 5 & 1989 \\
\hline & & & & Well 4 & WHC-SP-0480 & 1989 \\
\hline & & & & Well 4 & WHC-SP-0277 & 1988 \\
\hline & & & & Well 4 & UNI-3866 & 1986 \\
\hline \multirow[t]{16}{*}{ S2004 } & Y305 & NS-5 & 8.950 & Y305 & PNNL-14295, App. 2 & 2003 \\
\hline & & & & Y305 & PNNL-13910, App. 2 & 2002 \\
\hline & & & & C3458 & HEIS2_ADM_WELL & 2001 \\
\hline & & & & $177-\mathrm{N}-5$ & HEIS2_ADM_WELL & 2001 \\
\hline & & & & Y305 & PNNL-13487, App. 2 & 2001 \\
\hline & & & & Y305 & PNNL-13230, App. 2 & 2000 \\
\hline & & & & Y305 & PNNL-12088, App. 2 & 1999 \\
\hline & & & & Y305 & HNF-EP-0573-6 & 1998 \\
\hline & & & & Site 5 & HNF-EP-0573-5 & 1997 \\
\hline & & & & Site 5 & WHC-EP-0573-4 & 1996 \\
\hline & & & & NS-5 & WHC-SP-0098-8, Fig. A-54 & 1996 \\
\hline & & & & NS-5 & BHI-00164 & 1995 \\
\hline & & & & Site 5 & WHC-EP-0573-1 & 1993 \\
\hline & & & & Well 5 & WHC-SP-0480 & 1989 \\
\hline & & & & Well 5 & WHC-SP-0277 & 1988 \\
\hline & & & & Well 5 & UNI-3866 & 1986 \\
\hline \multirow[t]{12}{*}{ S2005 } & Y306 & NS-6 & 8.970 & Y306 & PNNL-14295, App. 2 & 2003 \\
\hline & & & & Y306 & PNNL-13910, App. 2 & 2002 \\
\hline & & & & C3459 & HEIS2_ADM_WELL & 2001 \\
\hline & & & & $177-\mathrm{N}-6$ & HEIS2_ADM_WELL & 2001 \\
\hline & & & & Y306 & PNNL-13487, App. 2 & 2001 \\
\hline & & & & Y306 & PNNL-13230, App. 2 & 2000 \\
\hline & & & & Y306 & PNNL-12088, App. 2 & 1999 \\
\hline & & & & Y306 & HNF-EP-0573-6 & 1998 \\
\hline & & & & Site 6 & HNF-EP-0573-5 & 1997 \\
\hline & & & & Site 6 & WHC-EP-0573-4 & 1996 \\
\hline & & & & NS-6 & WHC-SP-0098-8, Fig. A-54 & 1996 \\
\hline & & & & NS-6 & BHI-00164 & 1995 \\
\hline
\end{tabular}


Table 5.2. (contd)

\begin{tabular}{|c|c|c|c|c|c|c|}
\hline $\begin{array}{c}\text { Site } \\
\text { Identifier } \\
\text { (proposed) }\end{array}$ & $\begin{array}{l}\text { Spring Name } \\
\text { (use in 2009) }\end{array}$ & $\begin{array}{c}\text { Spring Name } \\
\text { (alternate use) }\end{array}$ & $\begin{array}{l}\text { HRM } \\
\text { ("2k") }\end{array}$ & $\begin{array}{c}\text { Name as Used in } \\
\text { Reference }\end{array}$ & Name Reference & $\begin{array}{l}\text { Year of } \\
\text { Name } \\
\text { Use }^{1}\end{array}$ \\
\hline & & & & Site 6 & WHC-EP-0573-1 & 1993 \\
\hline & & & & Well 6 & WHC-SP-0480 & 1989 \\
\hline & & & & Well 6 & WHC-SP-0277 & 1988 \\
\hline & & & & Well 6 & UNI-3866 & 1986 \\
\hline \multirow[t]{16}{*}{ S2006 } & Y307 & NS-7 & 8.985 & Y307 & PNNL-14295, App. 2 & 2003 \\
\hline & & & & Y307 & PNNL-13910, App. 2 & 2002 \\
\hline & & & & C3460 & HEIS2_ADM_WELL & 2001 \\
\hline & & & & $177-\mathrm{N}-7$ & HEIS2_ADM_WELL & 2001 \\
\hline & & & & Y307 & PNNL-13487, App. 2 & 2001 \\
\hline & & & & Y307 & PNNL-13230, App. 2 & 2000 \\
\hline & & & & Y307 & PNNL-12088, App. 2 & 1999 \\
\hline & & & & Y307 & HNF-EP-0573-6 & 1998 \\
\hline & & & & Site 7 & HNF-EP-0573-5 & 1997 \\
\hline & & & & Site 7 & WHC-EP-0573-4 & 1996 \\
\hline & & & & NS-7 & WHC-SP-0098-8, Fig. A-54 & 1996 \\
\hline & & & & NS-7 & BHI-00164 & 1995 \\
\hline & & & & Site 7 & WHC-EP-0573-1 & 1993 \\
\hline & & & & Well 7 & WHC-SP-0480 & 1989 \\
\hline & & & & Well 7 & WHC-SP-0277 & 1988 \\
\hline & & & & Well 7 & UNI-3866 & 1986 \\
\hline \multirow[t]{16}{*}{ S2007 } & Y308 & NS-8A & 9.000 & Y308 & PNNL-14295, App. 2 & 2003 \\
\hline & & & & Y308 & PNNL-13910, App. 2 & 2002 \\
\hline & & & & C3461 & HEIS2_ADM_WELL & 2001 \\
\hline & & & & $177-\mathrm{N}-8$ & HEIS2_ADM_WELL & 2001 \\
\hline & & & & Y308 & PNNL-13487, App. 2 & 2001 \\
\hline & & & & Y308 & PNNL-13230, App. 2 & 2000 \\
\hline & & & & Y308 & PNNL-12088, App. 2 & 1999 \\
\hline & & & & Y308 & HNF-EP-0573-6 & 1998 \\
\hline & & & & Site 8 & HNF-EP-0573-5 & 1997 \\
\hline & & & & Site 8 & WHC-EP-0573-4 & 1996 \\
\hline & & & & NS-8A & WHC-SP-0098-8, Fig. A-54 & 1996 \\
\hline & & & & NS-8A & BHI-00164 & 1995 \\
\hline & & & & Site 8 & WHC-EP-0573-1 & 1993 \\
\hline & & & & Well 8A & WHC-SP-0480 & 1989 \\
\hline & & & & Well 8A & WHC-SP-0277 & 1988 \\
\hline & & & & Well 8A & UNI-3866 & 1986 \\
\hline \multirow[t]{4}{*}{ S2008 } & NS-8B & NS-8B & 9.001 & NS-8B & BHI-00164 & 1995 \\
\hline & & & & C3462 & HEIS2_ADM_WELL & 2001 \\
\hline & & & & $177-\mathrm{N}-9$ & HEIS2_ADM_WELL & 2001 \\
\hline & & & & NS-8B & WHC-SP-0098-8, Fig. A-54 & 1996 \\
\hline \multirow[t]{7}{*}{ S2009 } & Y309 & NS-9 & 9.015 & Y309 & PNNL-14295, App. 2 & 2003 \\
\hline & & & & Y309 & PNNL-13910, App. 2 & 2002 \\
\hline & & & & C3463 & HEIS2_ADM_WELL & 2001 \\
\hline & & & & $177-\mathrm{N}-10$ & HEIS2_ADM_WELL & 2001 \\
\hline & & & & Y309 & PNNL-13487, App. 2 & 2001 \\
\hline & & & & Y309 & PNNL-13230, App. 2 & 2000 \\
\hline & & & & Y309 & PNNL-12088, App. 2 & 1999 \\
\hline
\end{tabular}


Table 5.2. (contd)

\begin{tabular}{|c|c|c|c|c|c|c|}
\hline $\begin{array}{c}\text { Site } \\
\text { Identifier } \\
\text { (proposed) }\end{array}$ & $\begin{array}{l}\text { Spring Name } \\
\text { (use in 2009) }\end{array}$ & $\begin{array}{c}\text { Spring Name } \\
\text { (alternate use) }\end{array}$ & $\begin{array}{c}\text { HRM } \\
\text { ("2k") }\end{array}$ & $\begin{array}{c}\text { Name as Used in } \\
\text { Reference }\end{array}$ & Name Reference & $\begin{array}{c}\text { Year of } \\
\text { Name } \\
\text { Use }^{1}\end{array}$ \\
\hline & & & & Y309 & HNF-EP-0573-6 & 1998 \\
\hline & & & & Site 9 & HNF-EP-0573-5 & 1997 \\
\hline & & & & Site 9 & WHC-EP-0573-4 & 1996 \\
\hline & & & & NS-9 & WHC-SP-0098-8, Fig. A-54 & 1996 \\
\hline & & & & NS-9 & BHI-00164 & 1995 \\
\hline & & & & Site 9 & WHC-EP-0573-1 & 1993 \\
\hline & & & & Well 9 & WHC-SP-0480 & 1989 \\
\hline & & & & Well 9 & WHC-SP-0277 & 1988 \\
\hline & & & & Well 9 & UNI-3866 & 1986 \\
\hline \multirow[t]{16}{*}{ S2010 } & Y310 & NS-10 & 9.030 & Y310 & PNNL-14295, App. 2 & 2003 \\
\hline & & & & Y310 & PNNL-13910, App. 2 & 2002 \\
\hline & & & & C3464 & HEIS2_ADM_WELL & 2001 \\
\hline & & & & $177-\mathrm{N}-11$ & HEIS2_ADM_WELL & 2001 \\
\hline & & & & Y310 & PNNL-13487, App. 2 & 2001 \\
\hline & & & & Y310 & PNNL-13230, App. 2 & 2000 \\
\hline & & & & Y310 & PNNL-12088, App. 2 & 1999 \\
\hline & & & & Y310 & HNF-EP-0573-6 & 1998 \\
\hline & & & & Site 10 & HNF-EP-0573-5 & 1997 \\
\hline & & & & Site 10 & WHC-EP-0573-4 & 1996 \\
\hline & & & & NS-10 & WHC-SP-0098-8, Fig. A-54 & 1996 \\
\hline & & & & NS-10 & BHI-00164 & 1995 \\
\hline & & & & Site 10 & WHC-EP-0573-1 & 1993 \\
\hline & & & & Well 10 & WHC-SP-0480 & 1989 \\
\hline & & & & Well 10 & WHC-SP-0277 & 1988 \\
\hline & & & & Well 10 & UNI-3866 & 1986 \\
\hline \multirow[t]{16}{*}{ S2011 } & Y311 & NS-11 & 9.100 & Y311 & PNNL-14295, App. 2 & 2003 \\
\hline & & & & Y311 & PNNL-13910, App. 2 & 2002 \\
\hline & & & & C3465 & HEIS2_ADM_WELL & 2001 \\
\hline & & & & $177-\mathrm{N}-12$ & HEIS2_ADM_WELL & 2001 \\
\hline & & & & Y311 & PNNL-13487, App. 2 & 2001 \\
\hline & & & & Y311 & PNNL-13230, App. 2 & 2000 \\
\hline & & & & Y311 & PNNL-12088, App. 2 & 1999 \\
\hline & & & & Y311 & HNF-EP-0573-6 & 1998 \\
\hline & & & & Site 11 & HNF-EP-0573-5 & 1997 \\
\hline & & & & Site 11 & WHC-EP-0573-4 & 1996 \\
\hline & & & & NS-11 & WHC-SP-0098-8, Fig. A-54 & 1996 \\
\hline & & & & NS-11 & BHI-00164 & 1995 \\
\hline & & & & Site 11 & WHC-EP-0573-1 & 1993 \\
\hline & & & & Well 11 & WHC-SP-0480 & 1989 \\
\hline & & & & Well 11 & WHC-SP-0277 & 1988 \\
\hline & & & & Well 11 & UNI-3866 & 1986 \\
\hline \multirow[t]{6}{*}{ S2012 } & Y312 & NS-12A & 9.125 & Y312 & PNNL-14295, App. 2 & 2003 \\
\hline & & & & Y312 & PNNL-13910, App. 2 & 2002 \\
\hline & & & & C3466 & HEIS2_ADM_WELL & 2001 \\
\hline & & & & $177-\mathrm{N}-13$ & HEIS2_ADM_WELL & 2001 \\
\hline & & & & Y312 & PNNL-13487, App. 2 & 2001 \\
\hline & & & & Y312 & PNNL-13230, App. 2 & 2000 \\
\hline
\end{tabular}


Table 5.2. (contd)

\begin{tabular}{|c|c|c|c|c|c|c|}
\hline $\begin{array}{c}\text { Site } \\
\text { Identifier } \\
\text { (proposed) }\end{array}$ & $\begin{array}{l}\text { Spring Name } \\
\text { (use in 2009) }\end{array}$ & $\begin{array}{c}\text { Spring Name } \\
\text { (alternate use) }\end{array}$ & $\begin{array}{c}\text { HRM } \\
\text { ("2k") }\end{array}$ & $\begin{array}{c}\text { Name as Used in } \\
\text { Reference }\end{array}$ & Name Reference & $\begin{array}{c}\text { Year of } \\
\text { Name } \\
\text { Use }^{1}\end{array}$ \\
\hline & & & & Y312 & PNNL-12088, App. 2 & 1999 \\
\hline & & & & Y312 & HNF-EP-0573-6 & 1998 \\
\hline & & & & Site 12 & HNF-EP-0573-5 & 1997 \\
\hline & & & & Site 12 & WHC-EP-0573-4 & 1996 \\
\hline & & & & NS-12A & WHC-SP-0098-8, Fig. A-54 & 1996 \\
\hline & & & & NS-12A & BHI-00164 & 1995 \\
\hline & & & & Site 12 & WHC-EP-0573-1 & 1993 \\
\hline & & & & Well 12A & WHC-SP-0480 & 1989 \\
\hline & & & & Well 12A & WHC-SP-0277 & 1988 \\
\hline & & & & Well 12A & UNI-3866 & 1986 \\
\hline \multirow[t]{4}{*}{ S2013 } & NS-12B & NS-12B & 9.126 & NS-12B & BHI-00164 & 1995 \\
\hline & & & & C3467 & HEIS2_ADM_WELL & 2001 \\
\hline & & & & $177-\mathrm{N}-14$ & HEIS2_ADM_WELL & 2001 \\
\hline & & & & NS-12B & WHC-SP-0098-8, Fig. A-54 & 1996 \\
\hline \multirow[t]{16}{*}{ S2014 } & Y313 & NS-13 & 9.140 & Y313 & PNNL-14295, App. 2 & 2003 \\
\hline & & & & Y313 & PNNL-13910, App. 2 & 2002 \\
\hline & & & & C3468 & HEIS2_ADM_WELL & 2001 \\
\hline & & & & $177-\mathrm{N}-15$ & HEIS2_ADM_WELL & 2001 \\
\hline & & & & Y313 & PNNL-13487, App. 2 & 2001 \\
\hline & & & & Y313 & PNNL-13230, App. 2 & 2000 \\
\hline & & & & Y313 & PNNL-12088, App. 2 & 1999 \\
\hline & & & & Y313 & HNF-EP-0573-6 & 1998 \\
\hline & & & & Site 13 & HNF-EP-0573-5 & 1997 \\
\hline & & & & Site 13 & WHC-EP-0573-4 & 1996 \\
\hline & & & & NS-13 & WHC-SP-0098-8, Fig. A-54 & 1996 \\
\hline & & & & NS-13 & BHI-00164 & 1995 \\
\hline & & & & Site 13 & WHC-EP-0573-1 & 1993 \\
\hline & & & & Well 13 & WHC-SP-0480 & 1989 \\
\hline & & & & Well 13 & WHC-SP-0277 & 1988 \\
\hline & & & & Well 13 & UNI-3866 & 1986 \\
\hline S2015 & SDW-110-1 & & 11.025 & SDW-110-1 & ERC-IOM \#040660 & 1996 \\
\hline
\end{tabular}





\subsection{Riverbed Pore Water Sampling Sites}

The upwelling of groundwater through riverbed substrate can be monitored by collecting samples of pore water (i.e., interstitial water) from bed sediment, although the logistics are extensive and sometimes dangerous. During 1995, a project was conducted using divers to insert small diameter sampling tubes into gravelly sediment in areas where groundwater containing chromium entered the river (Figure 6.1). The data from this project represent direct observation, rather than inference, of contaminant concentrations in the hyporheos that supports benthic habitat in the Hanford Reach.

\subsection{Riverbed Pore Water Site Naming Conventions}

The sampling naming convention adopted for the 1995 riverbed pore water sampling project is described in the project reports (Hope and Peterson 1996a, 1996b). For all sampling sites, the numbering convention started with a "T" to indicate a transect line extending offshore perpendicular to the shoreline. A steel pin was implanted at the shoreline to serve as the transect reference point. The second digit identified the reactor area: " $\mathrm{H}$ ” for $100-\mathrm{H}$, “D” for $100-\mathrm{D}$, and “K” for $100-\mathrm{K}$. (Note: Only one transect was completed at $100-\mathrm{K}$ before the project was cancelled).

At the 100-H Area, the sample site was further described by a transect number and distance offshore (Hope and Peterson 1996a). For example, site number "TH-3A" indicates transect number 3 at the 100-H Area, at distance A offshore (i.e., 9 meters). Distance B indicates 21 meters offshore.

For the 100-D Area sites, the naming convention was changed slightly by adding a "P" to the initial group of letters, signifying a pore water sample (Hope and Peterson 1996b). This was done to distinguish between pore water samples and river water samples (" $\mathrm{R}$ "), which were also collected at each riverbed site. Also, the " $\mathrm{A}$ " and " $\mathrm{B}$ " convention was changed from indicating distance offshore to indicating water depth (1.5 meter and 3 meters, respectively). A complete site name would be "TDP-17A," indicating transect 17 at the 100-D Area, pore water sample, and collected at depth A (1.5 meters). If a co-located sample was obtained for quality control purposes, a suffix “c” was added (TDP-17Ac).

In 1996, divers were again used to install six additional pore water sampling ports - two each at 100-D/DR, 100-H, and 100-K Areas (Environmental Restoration Contractor Interoffice Memorandum, MH Sturges to AJ Knepp, March 11, 1997, Installation of Riverbed Pore Water Sampling Ports in the Columbia River at 100-D/DR, 100-H, and 100-K Reactor Areas. Control No. 044041. Author: SJ Hope). These ports were connected to the high water shoreline by small diameter polyethylene tubing within a schedule 40 polyvinyl chloride overpack. Although very little use of these semi-permanent sampling facilities has occurred, a naming convention has been adopted that simply adds another "P" to the initial letter group, indicating permanent port (e.g., TDPP-15A).

Discreet identifiers for database purposes were not assigned to the sites sampled by the divers at the time of the project. The divers removed all equipment used to obtain the samples of sediment pore water, so it would not be possible to return to the exact location of sample collection. 


\subsection{Pore Water Sampling Site Names and Coordinates}

A listing of all riverbed pore water sampling sites visited under the Environmental Restoration program investigations in 1995 and 1996 is presented in Table 6.1. The fields in Table 6.1 are defined as follows:

- Site Identifier (proposed) - Identifier code proposed for each riverbed pore water sampling site. The code is used for internal data management purposes.

- Riverbed Site Name - The name used in the two published reports for the riverbed pore water sampling project (Hope and Peterson 1996a and 1996b).

- Sample Depth - Depth below ground surface (in feet) from which the pore water sample was collected.

- Segment - Segments defined for convenience in grouping monitoring sites by CERCLA groundwater operable units or other common characteristic.

- Northing and Easting - Location coordinates in Washington State Plane (South Zone) system; units are meters; North American Datum of 1983.

- Coordinate Quality - Quality of the coordinates, either "estimated” from various maps, or "surveyed" using global positioning system equipment (GPS) or traditional land surveying methods.

- Coordinate Type/Source - Source for coordinate information. “GPS deriv.” indicates that the sampling site coordinates were derived from a surveyed reference point. For the riverbed pore water projects, a steel pin was placed along the shoreline at each offshore diving transect. The direction and distance from the pin to the divers' location was recorded for each sampling site. The pins were surveyed using GPS equipment. “ARCmap” refers to GIS coverages for the river, including

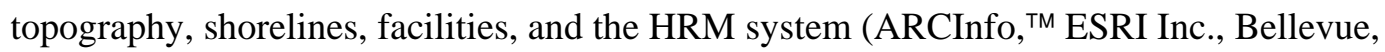
Washington). The GIS was used to position sites using narrative descriptions from field notes when GPS coordinates were not available.

- Coordinate Reference - Citation or project electronic file for information on the riverbed pore water sampling site.

- Comments - Notes verbatim from field notebooks.

\subsection{Pore Water Transect Reference Pins}

Table 6.2 lists the geographic coordinates for the reference pins used to locate the riverbed sites sampled by divers. The pins are steel rebar stakes driven into the cobbles along the rivershore, each fitted with a large orange-colored plastic cap. The columns are defined as follows:

- Segment - Segments defined for convenience in grouping monitoring sites by CERCLA groundwater operable units or other common characteristic.

- Transect Pin Identifier - Name assigned to steel pins (rebar) used as reference points for each of the offshore transects of riverbed pore water sampling tube locations.

- Transect Pin Field Label - Name attached to steel pin in the field during the investigations. 
- GPS ID (ERC) - Identifier assigned by Bechtel Hanford, Inc. to each location surveyed using GPS equipment. Data from GPS surveys conducted by Bechtel are stored in the Hanford Geographic Information System (HGIS).

- Pin Northing and Pin Easting - Geographic coordinates for the pin referenced to the Washington State Plane system, South Zone; units are meters; North American Datum of 1983.

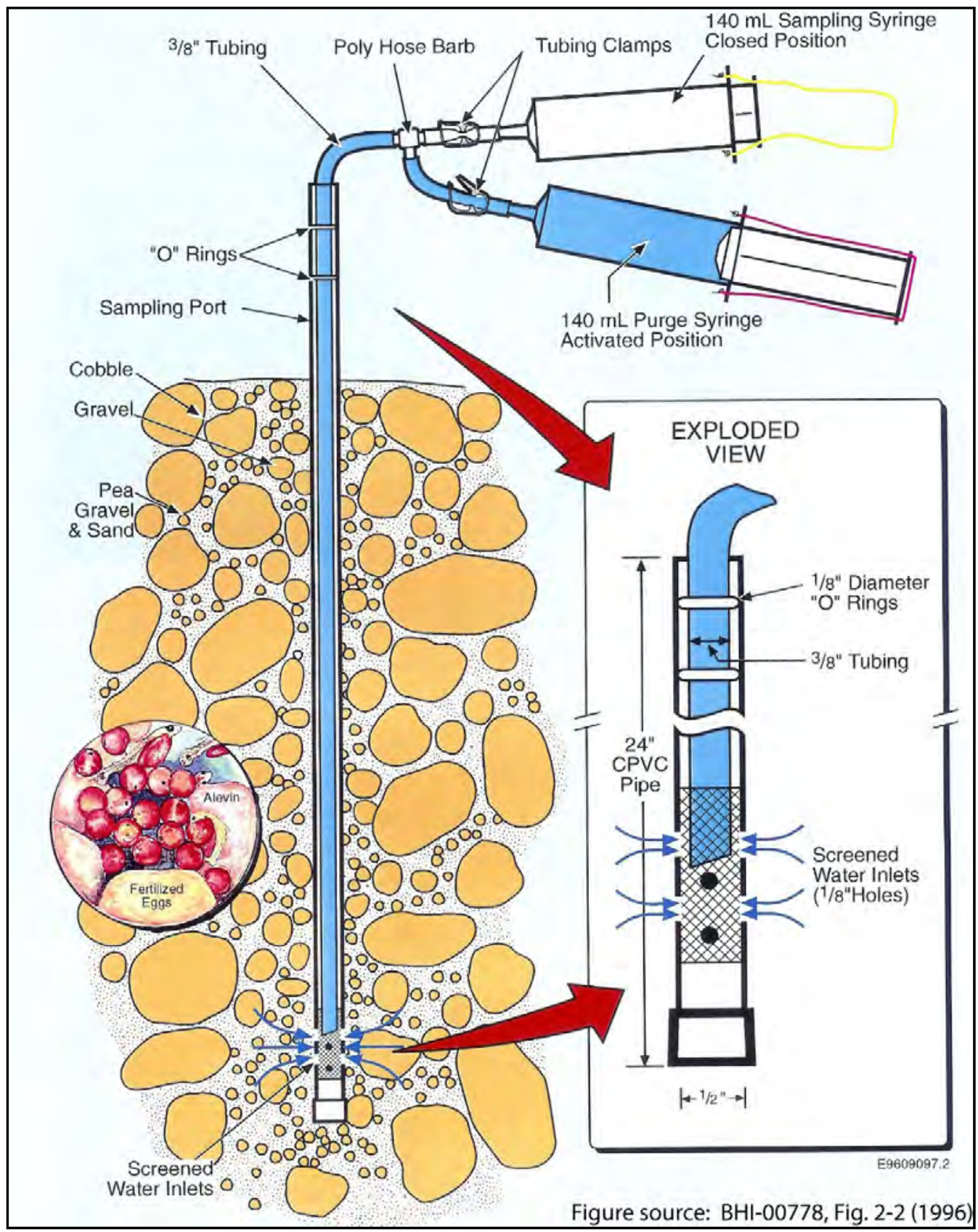

Figure 6.1. Diagram of Riverbed Pore Water Sampling Apparatus 
Table 6.1. Riverbed Substrate Pore Water Sampling Sites Along the Hanford Site Shoreline

\begin{tabular}{|c|c|c|c|c|c|c|c|c|c|}
\hline $\begin{array}{c}\text { Site } \\
\text { Identifier } \\
\text { (proposed) }\end{array}$ & $\begin{array}{c}\text { Riverbed } \\
\text { Site Name }\end{array}$ & $\begin{array}{c}\text { Sample } \\
\text { Depth } \\
\text { (ft-bgs) }\end{array}$ & Segment $^{1}$ & $\begin{array}{c}\text { Northing } \\
\text { (m-NAD83) }\end{array}$ & $\begin{array}{c}\text { Easting } \\
\text { (m-NAD83) }\end{array}$ & $\begin{array}{c}\text { Coordinate } \\
\text { Quality }^{2}\end{array}$ & $\begin{array}{c}\text { Coordinate } \\
\text { Type/Source } \\
\end{array}$ & $\begin{array}{c}\text { Coordinate } \\
\text { Reference }\end{array}$ & Comments \\
\hline P1001 & TH-01A & 1.5 & $\mathrm{HR} 3 \mathrm{H}$ & $152,732.228$ & $578,208.895$ & SURV & GPS deriv. & BHI-00345 & \\
\hline P1002 & TH-01B & 1.5 & HR3H & $152,736.800$ & $578,200.976$ & SURV & GPS deriv. & BHI-00345 & \\
\hline P1003 & TH-02A & 1.5 & $\mathrm{HR} 3 \mathrm{H}$ & $152,791.316$ & $578,169.898$ & SURV & GPS deriv. & BHI-00345 & \\
\hline P1004 & TH-02B & 1.5 & $\mathrm{HR} 3 \mathrm{H}$ & $152,798.134$ & $578,159.790$ & SURV & GPS deriv. & BHI-00345 & \\
\hline P1005 & TH-03A & 1.5 & $\mathrm{HR} 3 \mathrm{H}$ & $152,845.605$ & $578,175.004$ & SURV & GPS deriv. & BHI-00345 & \\
\hline P1006 & TH-03B & 1.5 & HR3H & $152,852.943$ & $578,165.267$ & SURV & GPS deriv. & BHI-00345 & \\
\hline P1007 & TH-04A & 1.5 & $\mathrm{HR} 3 \mathrm{H}$ & $152,904.172$ & $578,152.989$ & SURV & GPS deriv. & BHI-00345 & \\
\hline P1008 & TH-04B & 1.5 & HR3H & $152,905.639$ & $578,151.042$ & SURV & GPS deriv. & BHI-00345 & \\
\hline P1009 & TH-05A & 1.5 & $\mathrm{HR} 3 \mathrm{H}$ & $152,966.674$ & $578,123.881$ & SURV & GPS deriv. & BHI-00345 & \\
\hline P1010 & TH-05B & 1.5 & HR3H & $152,974.561$ & $578,113.413$ & SURV & GPS deriv. & BHI-00345 & \\
\hline P1011 & TH-06A & 1.5 & $\mathrm{HR} 3 \mathrm{H}$ & $153,017.723$ & $578,090.511$ & SURV & GPS deriv. & BHI-00345 & \\
\hline P1012 & TH-06B & 1.5 & HR3H & $153,025.610$ & $578,080.043$ & SURV & GPS deriv. & BHI-00345 & \\
\hline P1013 & TH-07A & 1.5 & $\mathrm{HR} 3 \mathrm{H}$ & $153,067.463$ & $578,061.403$ & SURV & GPS deriv. & BHI-00345 & \\
\hline P1014 & TH-07B & 1.5 & HR3H & $153,075.825$ & $578,052.116$ & SURV & GPS deriv. & BHI-00345 & \\
\hline P1015 & TH-08A & 1.5 & $\mathrm{HR} 3 \mathrm{H}$ & $153,116.737$ & $578,026.432$ & SURV & GPS deriv. & BHI-00345 & \\
\hline P1016 & TH-08B & 1.5 & $\mathrm{HR} 3 \mathrm{H}$ & $153,120.408$ & $578,022.354$ & SURV & GPS deriv. & BHI-00345 & \\
\hline P1017 & TH-09A & 1.5 & HR3H & $153,168.886$ & $577,986.459$ & SURV & GPS deriv. & BHI-00345 & \\
\hline P1018 & TH-09B & 1.5 & $\mathrm{HR} 3 \mathrm{H}$ & $153,173.372$ & $577,981.475$ & SURV & GPS deriv. & BHI-00345 & \\
\hline P1019 & $\mathrm{TH}-10 \mathrm{~A}$ & 1.5 & $\mathrm{HR} 3 \mathrm{H}$ & $153,212.878$ & $577,948.760$ & SURV & GPS deriv. & BHI-00345 & \\
\hline P1020 & TH-10B & 1.5 & $\mathrm{HR} 3 \mathrm{H}$ & $153,217.365$ & $577,943.776$ & SURV & GPS deriv. & BHI-00345 & \\
\hline P1021 & TH-11A & 1.5 & $\mathrm{HR} 3 \mathrm{H}$ & $153,254.251$ & $577,904.310$ & SURV & GPS deriv. & BHI-00345 & \\
\hline P1022 & TH-11B & 1.5 & $\mathrm{HR} 3 \mathrm{H}$ & $153,263.390$ & $577,895.787$ & SURV & GPS deriv. & BHI-00345 & \\
\hline P1023 & TH-12A & 1.5 & $\mathrm{HR} 3 \mathrm{H}$ & $153,300.227$ & $577,867.328$ & SURV & GPS deriv. & BHI-00345 & \\
\hline P1024 & TH-12B & 1.5 & $\mathrm{HR} 3 \mathrm{H}$ & $153,305.354$ & $577,862.547$ & SURV & GPS deriv. & BHI-00345 & \\
\hline P1025 & TH-13A & 1.5 & $\mathrm{HR} 3 \mathrm{H}$ & $153,345.550$ & $577,825.384$ & SURV & GPS deriv. & BHI-00345 & \\
\hline P1026 & $\mathrm{TH}-13 \mathrm{~B}$ & 1.5 & $\mathrm{HR} 3 \mathrm{H}$ & $153,350.677$ & $577,820.603$ & SURV & GPS deriv. & BHI-00345 & \\
\hline P1027 & TH-14A & 1.5 & $\mathrm{HR} 3 \mathrm{H}$ & $153,383.622$ & $577,784.674$ & SURV & GPS deriv. & BHI-00345 & \\
\hline P1028 & TH-14B & 1.5 & $\mathrm{HR} 3 \mathrm{H}$ & $153,390.363$ & $577,779.954$ & SURV & GPS deriv. & BHI-00345 & \\
\hline P1029 & TH-15A & 1.5 & HR3H & $153,421.543$ & $577,731.286$ & SURV & GPS deriv. & BHI-00345 & \\
\hline P1030 & TH-15B & & $\mathrm{HR} 3 \mathrm{H}$ & $153,412.804$ & $577,737.405$ & SURV & GPS deriv. & BHI-00345 & Not sampled \\
\hline P1031 & TH-16A & 1.5 & $\mathrm{HR} 3 \mathrm{H}$ & $153,457.672$ & $577,676.872$ & SURV & GPS deriv. & BHI-00345 & \\
\hline P1032 & TH-16B & & $\mathrm{HR} 3 \mathrm{H}$ & $153,451.180$ & $577,681.417$ & SURV & GPS deriv. & BHI-00345 & Not sampled \\
\hline P1033 & TH-17A & 1.5 & HR3H & $153,487.252$ & $577,636.467$ & SURV & GPS deriv. & BHI-00345 & \\
\hline
\end{tabular}


Table 6.1. (contd)

\begin{tabular}{|c|c|c|c|c|c|c|c|c|c|}
\hline $\begin{array}{c}\text { Site } \\
\text { Identifier } \\
\text { (proposed) }\end{array}$ & $\begin{array}{l}\text { Riverbed } \\
\text { Site Name }\end{array}$ & $\begin{array}{c}\text { Sample } \\
\text { Depth } \\
\text { (ft-bgs) }\end{array}$ & Segment $^{1}$ & $\begin{array}{c}\text { Northing } \\
\text { (m-NAD83) }\end{array}$ & $\begin{array}{c}\text { Easting } \\
\text { (m-NAD83) }\end{array}$ & $\begin{array}{c}\text { Coordinate } \\
\text { Quality }^{2}\end{array}$ & $\begin{array}{l}\text { Coordinate } \\
\text { Type/Source }\end{array}$ & $\begin{array}{l}\text { Coordinate } \\
\text { Reference }\end{array}$ & Comments \\
\hline P1034 & TH-17B & & $\mathrm{HR} 3 \mathrm{H}$ & $153,478.763$ & $577,642.411$ & SURV & GPS deriv. & BHI-00345 & Not sampled \\
\hline P1035 & TH-18A & & $\mathrm{HR} 3 \mathrm{H}$ & $152,669.622$ & $578,252.554$ & SURV & GPS deriv. & $\mathrm{BHI}-00345$ & Not sampled \\
\hline P1036 & TH-18B & & $\mathrm{HR} 3 \mathrm{H}$ & $152,669.622$ & $578,252.554$ & SURV & GPS deriv. & BHI-00345 & Not sampled \\
\hline P1037 & TH-19A & & $\mathrm{HR} 3 \mathrm{H}$ & $152,621.806$ & $578,290.208$ & SURV & GPS deriv. & BHI-00345 & Not sampled \\
\hline P1038 & TH-19B & & $\mathrm{HR} 3 \mathrm{H}$ & $152,621.806$ & $578,290.208$ & SURV & GPS deriv. & BHI-00345 & Not sampled \\
\hline P1039 & TH-20A & & $\mathrm{HR} 3 \mathrm{H}$ & $152,577.402$ & $578,332.138$ & SURV & GPS deriv. & $\mathrm{BHI}-00345$ & Not sampled \\
\hline P1040 & TH-20B & & $\mathrm{HR} 3 \mathrm{H}$ & $152,577.402$ & $578,332.138$ & SURV & GPS deriv. & $\mathrm{BHI}-00345$ & Not sampled \\
\hline P1041 & $\mathrm{TH}-21 \mathrm{~A}$ & & $\mathrm{HR} 3 \mathrm{H}$ & $152,539.336$ & $578,379.518$ & SURV & GPS deriv. & BHI-00345 & Not sampled \\
\hline P1042 & TH-21B & & $\mathrm{HR} 3 \mathrm{H}$ & $152,539.336$ & $578,379.518$ & SURV & GPS deriv. & BHI-00345 & Not sampled \\
\hline P1043 & TH-22A & & $\mathrm{HR} 3 \mathrm{H}$ & $152,499.373$ & $578,425.129$ & SURV & GPS deriv. & $\mathrm{BHI}-00345$ & Not sampled \\
\hline P1044 & TH-22B & & $\mathrm{HR} 3 \mathrm{H}$ & $152,499.373$ & $578,425.129$ & SURV & GPS deriv. & $\mathrm{BHI}-00345$ & Not sampled \\
\hline P1045 & TV-26A & 1.5 & VB & $144,029.791$ & $550,978.256$ & EST & ARCmap & BHI-00345 & Background sites for $\mathrm{Cr}+6$ study \\
\hline P1046 & TV-26B & 1.5 & VB & $143,983.403$ & $550,978.256$ & EST & ARCmap & $\mathrm{BHI}-00345$ & Background sites for $\mathrm{Cr}+6$ study \\
\hline P1047 & TV-27A & 1.5 & VB & $144,033.359$ & $550,441.220$ & EST & ARCmap & BHI-00345 & Background sites for $\mathrm{Cr}+6$ study \\
\hline P1048 & TV-27B & 1.5 & VB & $143,995.892$ & $550,441.220$ & EST & ARCmap & $\mathrm{BHI}-00345$ & Background sites for $\mathrm{Cr}+6$ study \\
\hline P1049 & TV-28A & 1.5 & VB & $144,049.417$ & $549,870.285$ & EST & ARCmap & $\mathrm{BHI}-00345$ & Background sites for $\mathrm{Cr}+6$ study \\
\hline P1050 & TV-28B & 1.5 & VB & $144,015.518$ & $549,870.285$ & EST & ARCmap & $\mathrm{BHI}-00345$ & Background sites for $\mathrm{Cr}+6$ study \\
\hline P2001 & TDP-01A & 1.5 & HR3D & $153,307.320$ & $574,041.452$ & SURV & GPS deriv. & BHI-00778 & Azi estimate (poresurv cmt) \\
\hline P2002 & TDP-01B & & HR3D & $153,313.575$ & $574,024.267$ & SURV & GPS deriv. & $\mathrm{BHI}-00778$ & Not sampled \\
\hline P2003 & TDP-02A & 1.5 & HR3D & $153,255.351$ & $574,007.581$ & SURV & GPS deriv. & $\mathrm{BHI}-00778$ & Azi estimate (poresurv cmt) \\
\hline P2004 & TDP-02B & & HR3D & $153,262.649$ & $573,987.531$ & SURV & GPS deriv. & BHI-00778 & Not sampled \\
\hline P2005 & TDP-03A & 1.5 & HR3D & $153,201.632$ & $573,979.142$ & SURV & GPS deriv. & BHI-00778 & Azi estimate (poresurv cmt) \\
\hline P2006 & TDP-03B & & HR3D & $153,209.138$ & $573,958.519$ & SURV & GPS deriv. & BHI-00778 & Not sampled \\
\hline P2007 & TDP-04A & & HR3D & $153,144.379$ & $573,958.843$ & SURV & GPS deriv. & BHI-00778 & Estimate (poresurv cmt) \\
\hline P2008 & TDP-04B & & HR3D & $153,152.197$ & $573,937.361$ & SURV & GPS deriv. & BHI-00778 & Not sampled \\
\hline P2009 & TDP-05A & 1.5 & HR3D & $153,087.985$ & $573,932.759$ & SURV & GPS deriv. & BHI-00778 & \\
\hline P2010 & TDP-05B & & HR3D & $153,093.866$ & $573,913.522$ & SURV & GPS deriv. & BHI-00778 & Not sampled \\
\hline P2011 & TDP-06A & 1.5 & HR3D & $153,028.857$ & $573,925.835$ & SURV & GPS deriv. & BHI-00778 & \\
\hline P2012 & TDP-06B & 0.8 & HR3D & $153,026.673$ & $573,914.134$ & SURV & GPS deriv. & BHI-00778 & \\
\hline P2013 & TDP-07A & 1.5 & HR3D & $152,999.309$ & $573,884.756$ & SURV & GPS deriv. & BHI-00778 & \\
\hline P2014 & TDP-07B & 1.5 & HR3D & $152,968.408$ & $573,905.926$ & SURV & GPS deriv. & BHI-00778 & \\
\hline P2015 & TDP-08A & 1.5 & HR3D & $152,913.038$ & $573,892.795$ & SURV & GPS deriv. & BHI-00778 & \\
\hline
\end{tabular}


Table 6.1. (contd)

\begin{tabular}{|c|c|c|c|c|c|c|c|c|c|}
\hline $\begin{array}{c}\text { Site } \\
\text { Identifier } \\
\text { (proposed) }\end{array}$ & $\begin{array}{c}\text { Riverbed } \\
\text { Site Name } \\
\end{array}$ & $\begin{array}{c}\text { Sample } \\
\text { Depth } \\
\text { (ft-bgs) }\end{array}$ & Segment $^{1}$ & $\begin{array}{c}\text { Northing } \\
\text { (m-NAD83) }\end{array}$ & $\begin{array}{c}\text { Easting } \\
\text { (m-NAD83) }\end{array}$ & $\begin{array}{c}\text { Coordinate } \\
\text { Quality }^{2}\end{array}$ & \begin{tabular}{|c|} 
Coordinate \\
Type/Source \\
\end{tabular} & $\begin{array}{c}\text { Coordinate } \\
\text { Reference }\end{array}$ & Comments \\
\hline P2016 & TDP-08B & 1.0 & HR3D & $152,915.036$ & $573,879.194$ & SURV & GPS deriv. & BHI-00778 & \\
\hline P2017 & TDP-09A & 1.5 & HR3D & $152,852.385$ & $573,877.768$ & SURV & GPS deriv. & $\mathrm{BHI}-00778$ & \\
\hline P2018 & TDP-09B & 1.3 & HR3D & $152,858.985$ & $573,861.362$ & SURV & GPS deriv. & BHI-00778 & \\
\hline P2019 & TDP-09BC & 0.8 & HR3D & $152,862.985$ & $573,865.362$ & SURV & GPS deriv. & $\mathrm{BHI}-00778$ & Colocate $+4 \mathrm{~m}$ east and north \\
\hline P2020 & TDP-10A & 1.5 & HR3D & $152,796.585$ & $573,856.023$ & SURV & GPS deriv. & BHI-00778 & \\
\hline P2021 & TDP-10B & 1.5 & HR3D & $152,801.652$ & $573,844.588$ & SURV & GPS deriv. & $\mathrm{BHI}-00778$ & \\
\hline P2022 & TDP-11A & 1.5 & HR3D & $152,755.155$ & $573,818.291$ & SURV & GPS deriv. & BHI-00778 & \\
\hline P2023 & TDP-11B & 1.5 & HR3D & $152,751.267$ & $573,815.963$ & SURV & GPS deriv. & $\mathrm{BHI}-00778$ & \\
\hline P2024 & TDP-12A & 1.5 & HR3D & $152,701.972$ & $573,797.501$ & SURV & GPS deriv. & BHI-00778 & \\
\hline P2025 & TDP-12B & 1.5 & HR3D & $152,699.958$ & $573,790.916$ & SURV & GPS deriv. & $\mathrm{BHI}-00778$ & \\
\hline P2026 & TDP-13A & 1.5 & HR3D & $152,650.961$ & $573,766.722$ & SURV & GPS deriv. & $\mathrm{BHI}-00778$ & \\
\hline P2027 & TDP-13AC & 1.5 & HR3D & $152,654.961$ & $573,770.722$ & SURV & GPS deriv. & $\mathrm{BHI}-00778$ & Colocate $+4 \mathrm{~m}$ east and north \\
\hline P2028 & TDP-13B & 1.5 & HR3D & $152,664.709$ & $573,745.026$ & SURV & GPS deriv. & BHI-00778 & \\
\hline P2029 & TDP-14A & 1.5 & HR3D & $152,605.102$ & $573,729.531$ & SURV & GPS deriv. & $\mathrm{BHI}-00778$ & \\
\hline P2030 & TDP-14B & 1.5 & HR3D & $152,616.670$ & $573,710.924$ & SURV & GPS deriv. & BHI-00778 & \\
\hline P2031 & TDP-15A & 1.5 & HR3D & $152,561.596$ & $573,691.908$ & SURV & GPS deriv. & BHI-00778 & \\
\hline P2032 & TDP-15B & 1.5 & HR3D & $152,583.014$ & $573,678.659$ & SURV & GPS deriv. & BHI-00778 & \\
\hline P2033 & TDP-15Bc & 1.5 & HR3D & $152,587.014$ & $573,682.659$ & SURV & GPS deriv. & BHI-00778 & Colocate $+4 \mathrm{~m}$ east and north \\
\hline P2034 & TDP-16A & 1.5 & HR3D & $152,532.307$ & $573,638.001$ & SURV & GPS deriv. & BHI-00778 & \\
\hline P2035 & TDP-16B & 1.5 & HR3D & $152,546.506$ & $573,629.373$ & SURV & GPS deriv. & BHI-00778 & \\
\hline P2036 & TDP-17A & 1.5 & HR3D & $152,490.782$ & $573,590.821$ & SURV & GPS deriv. & BHI-00778 & \\
\hline P2037 & TDP-17B & 1.5 & HR3D & $152,503.899$ & $573,579.884$ & SURV & GPS deriv. & $\mathrm{BHI}-00778$ & \\
\hline P2038 & TDP-18A & 1.5 & HR3D & $152,462.817$ & $573,542.065$ & SURV & GPS deriv. & BHI-00778 & \\
\hline P2039 & TDP-18AC & 1.5 & HR3D & $152,466.817$ & $573,546.065$ & SURV & GPS deriv. & BHI-00778 & Colocate $+4 \mathrm{~m}$ east and north \\
\hline P2040 & TDP-18B & 1.3 & HR3D & $152,467.882$ & $573,530.880$ & SURV & GPS deriv. & BHI-00778 & \\
\hline P2041 & TDP-19A & 1.5 & HR3D & $152,380.000$ & $573,490.000$ & SURV & GPS deriv. & $\mathrm{BHI}-00778$ & Not in GPS survey list \\
\hline P2042 & TDP-19B & 1.3 & HR3D & $152,395.000$ & $573,475.000$ & SURV & GPS deriv. & BHI-00778 & Not in GPS survey list \\
\hline P2043 & TDP-19Bc & 0.8 & HR3D & $152,399.000$ & $573,479.000$ & SURV & GPS deriv. & $\mathrm{BHI}-00778$ & Not in GPS survey list; colocate \\
\hline P2044 & TDP-20A & 1.5 & HR3D & $152,388.174$ & $573,432.948$ & SURV & GPS deriv. & BHI-00778 & \\
\hline P2045 & TDP-20B & 1.5 & HR3D & $152,390.462$ & $573,434.652$ & SURV & GPS deriv. & $\mathrm{BHI}-00778$ & \\
\hline P2046 & TDP-21A & 1.5 & HR3D & $152,362.855$ & $573,394.522$ & SURV & GPS deriv. & BHI-00778 & \\
\hline P2047 & TDP-21B & 1.5 & HR3D & $152,355.013$ & $573,388.964$ & SURV & GPS deriv. & BHI-00778 & \\
\hline
\end{tabular}


Table 6.1. (contd)

\begin{tabular}{|c|c|c|c|c|c|c|c|c|c|}
\hline $\begin{array}{c}\text { Site } \\
\text { Identifier } \\
\text { (proposed) }\end{array}$ & $\begin{array}{l}\text { Riverbed } \\
\text { Site Name }\end{array}$ & $\begin{array}{c}\text { Sample } \\
\text { Depth } \\
\text { (ft-bgs) }\end{array}$ & Segment $^{1}$ & $\begin{array}{c}\text { Northing } \\
\text { (m-NAD83) }\end{array}$ & $\begin{array}{c}\text { Easting } \\
\text { (m-NAD83) }\end{array}$ & $\begin{array}{l}\text { Coordinate } \\
\text { Quality }^{2}\end{array}$ & $\begin{array}{l}\text { Coordinate } \\
\text { Type/Source }\end{array}$ & $\begin{array}{c}\text { Coordinate } \\
\text { Reference }\end{array}$ & Comments \\
\hline P2048 & TDP-22A & 1.5 & HR3D & $152,320.921$ & $573,348.062$ & SURV & GPS deriv. & BHI-00778 & \\
\hline P2049 & TDP-22B & 1.3 & HR3D & $152,324.934$ & $573,338.772$ & SURV & GPS deriv. & BHI-00778 & \\
\hline P2050 & TDP-23A & 1.3 & HR3D & $152,293.394$ & $573,296.902$ & SURV & GPS deriv. & BHI-00778 & \\
\hline P2051 & TDP-23B & 1.2 & HR3D & $152,311.636$ & $573,276.577$ & SURV & GPS deriv. & BHI-00778 & \\
\hline P2052 & TDP-24A & 1.5 & HR3D & $152,257.185$ & $573,243.436$ & SURV & GPS deriv. & BHI-00778 & \\
\hline P2053 & TDP-24B & 1.5 & HR3D & $152,275.394$ & $573,231.745$ & SURV & GPS deriv. & BHI-00778 & \\
\hline P2054 & TDP-25A & 1.5 & HR3D & $152,213.693$ & $573,193.813$ & SURV & GPS deriv. & BHI-00778 & \\
\hline P2055 & TDP-25B & 1.2 & HR3D & $152,229.612$ & $573,187.445$ & SURV & GPS deriv. & BHI-00778 & \\
\hline P2056 & TDP-26A & 1.5 & HR3D & $152,183.062$ & $573,137.300$ & SURV & GPS deriv. & BHI-00778 & \\
\hline P2057 & TDP-26B & 1.5 & HR3D & $152,194.479$ & $573,136.029$ & SURV & GPS deriv. & BHI-00778 & \\
\hline P2058 & TDP-27A & 1.5 & HR3D & $152,145.394$ & $573,092.755$ & SURV & GPS deriv. & BHI-00778 & \\
\hline P2059 & TDP-27B & 1.5 & HR3D & $152,156.111$ & $573,087.141$ & SURV & GPS deriv. & BHI-00778 & \\
\hline P2060 & TDP-27Bc & 1.5 & HR3D & $152,160.111$ & $573,091.141$ & SURV & GPS deriv. & $\mathrm{BHI}-00778$ & Colocate $+4 \mathrm{~m}$ east and north \\
\hline P2061 & TDP-28A & 0.8 & HR3D & $152,105.697$ & $573,047.953$ & SURV & GPS deriv. & BHI-00778 & \\
\hline P2062 & TDP-28B & 1.2 & HR3D & $152,112.980$ & $573,036.511$ & SURV & GPS deriv. & $\mathrm{BHI}-00778$ & \\
\hline P2063 & TDP-29A & 1.5 & HR3D & $152,081.837$ & $572,994.625$ & SURV & GPS deriv. & $\mathrm{BHI}-00778$ & \\
\hline P2064 & TDP-29B & & HR3D & $152,088.932$ & $572,981.860$ & SURV & GPS deriv. & $\mathrm{BHI}-00778$ & Not sampled \\
\hline P2065 & TDP-30A & 0.8 & HR3D & $152,038.827$ & $572,944.146$ & SURV & GPS deriv. & BHI-00778 & \\
\hline P2066 & TDP-30B & 1.5 & HR3D & $152,060.476$ & $572,936.006$ & SURV & GPS deriv. & $\mathrm{BHI}-00778$ & \\
\hline P2067 & TDP-31A & 1.5 & HR3D & $151,974.878$ & $572,919.805$ & SURV & GPS deriv. & BHI-00778 & \\
\hline P2068 & TDP-31B & 1.5 & HR3D & $151,977.550$ & $572,917.771$ & SURV & GPS deriv. & BHI-00778 & \\
\hline P2069 & TDP-32A & 1.5 & HR3D & $151,914.971$ & $572,898.020$ & SURV & GPS deriv. & BHI-00778 & \\
\hline P2070 & TDP-32B & 1.5 & HR3D & $151,940.630$ & $572,868.703$ & SURV & GPS deriv. & $\mathrm{BHI}-00778$ & \\
\hline P2071 & TDP-33A & 1.5 & HR3D & $151,871.775$ & $572,861.603$ & SURV & GPS deriv. & BHI-00778 & \\
\hline P2072 & TDP-33Ac & 1.5 & HR3D & $151,875.775$ & $572,865.603$ & SURV & GPS deriv. & BHI-00778 & Colocate $+4 m$ east and north \\
\hline P2073 & TDP-33B & 1.2 & HR3D & $151,904.321$ & $572,831.253$ & SURV & GPS deriv. & BHI-00778 & \\
\hline P2074 & TDP-34A & 1.5 & HR3D & $151,834.455$ & $572,812.185$ & SURV & GPS deriv. & $\mathrm{BHI}-00778$ & \\
\hline P2075 & TDP-34B & 1.5 & HR3D & $151,867.879$ & $572,792.417$ & SURV & GPS deriv. & BHI-00778 & \\
\hline P2076 & TDP-38A & 1.5 & HR3D & $151,687.207$ & $572,628.624$ & SURV & GPS deriv. & $\mathrm{BHI}-00778$ & \\
\hline P2077 & TDP-38B & 1.5 & HR3D & $151,707.580$ & $572,595.520$ & SURV & GPS deriv. & $\mathrm{BHI}-00778$ & \\
\hline P2078 & TDP-39A & 1.5 & HR3D & $151,634.234$ & $572,596.074$ & SURV & GPS deriv. & $\mathrm{BHI}-00778$ & \\
\hline P2079 & TDP-39Ac & 1.5 & HR3D & $151,638.234$ & $572,600.074$ & SURV & GPS deriv. & BHI-00778 & Colocate $+4 \mathrm{~m}$ east and north \\
\hline
\end{tabular}


Table 6.1. (contd)

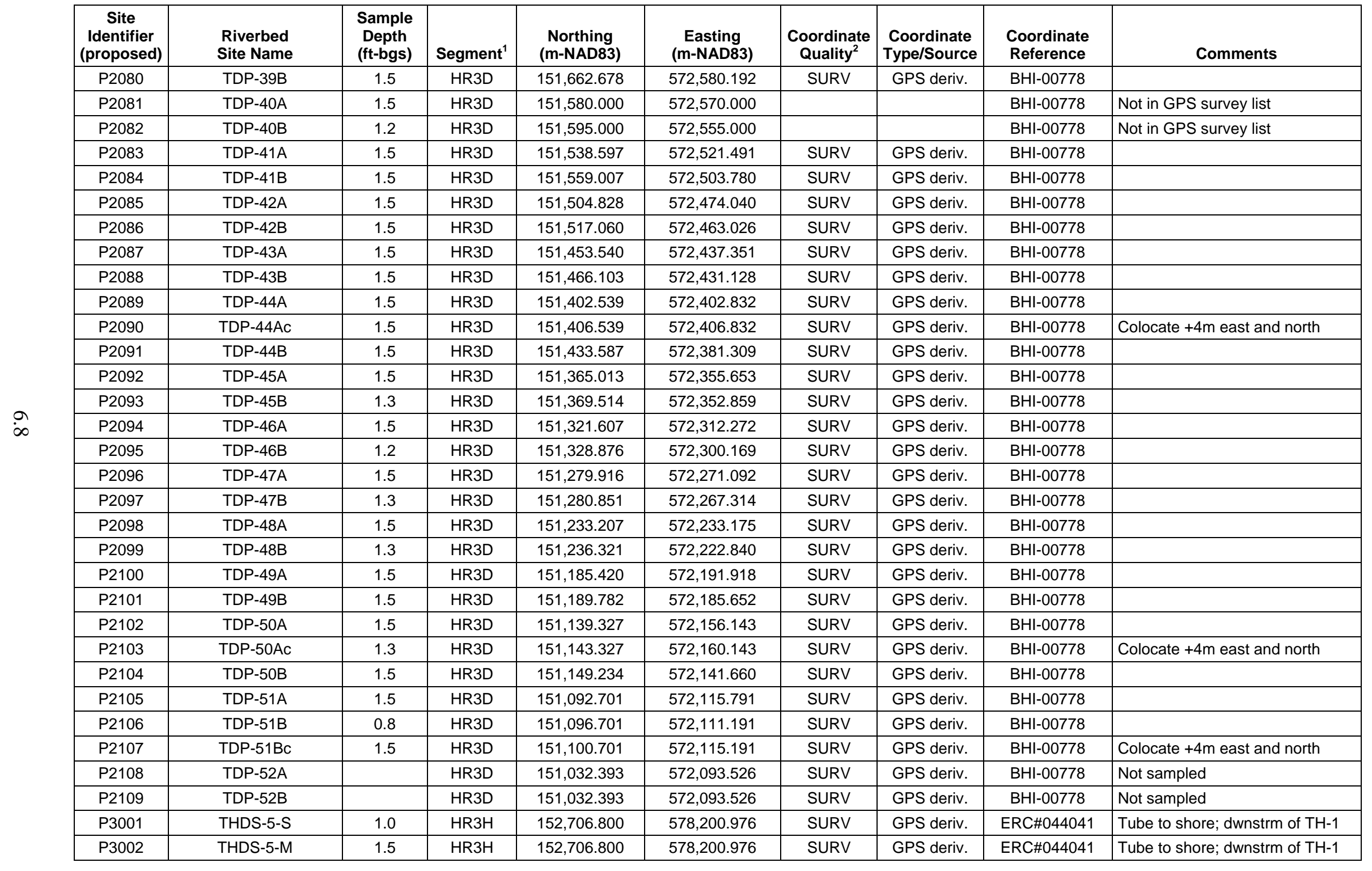


Table 6.1. (contd)

\begin{tabular}{|c|c|c|c|c|c|c|c|c|c|}
\hline $\begin{array}{c}\text { Site } \\
\text { Identifier } \\
\text { (proposed) }\end{array}$ & $\begin{array}{l}\text { Riverbed } \\
\text { Site Name }\end{array}$ & $\begin{array}{c}\text { Sample } \\
\text { Depth } \\
\text { (ft-bgs) }\end{array}$ & Segment $^{1}$ & $\begin{array}{c}\text { Northing } \\
\text { (m-NAD83) }\end{array}$ & $\begin{array}{c}\text { Easting } \\
\text { (m-NAD83) }\end{array}$ & $\begin{array}{l}\text { Coordinate } \\
\text { Quality }^{2}\end{array}$ & $\begin{array}{l}\text { Coordinate } \\
\text { Type/Source }\end{array}$ & $\begin{array}{l}\text { Coordinate } \\
\text { Reference }\end{array}$ & Comments \\
\hline P3003 & THDS-5-D & 2.0 & $\mathrm{HR} 3 \mathrm{H}$ & $152,706.800$ & $578,200.976$ & SURV & GPS deriv. & ERC\#044041 & Tube to shore; dwnstrm of TH-1 \\
\hline P3004 & TH-01B-S & 1.0 & $\mathrm{HR} 3 \mathrm{H}$ & $152,736.800$ & $578,200.976$ & SURV & GPS deriv. & ERC\#044041 & Tube to shore \\
\hline P3005 & TH-01B-M & 1.5 & $\mathrm{HR} 3 \mathrm{H}$ & $152,736.800$ & $578,200.976$ & SURV & GPS deriv. & ERC\#044041 & Tube to shore \\
\hline P3006 & TH-01B-D & 2.0 & $\mathrm{HR} 3 \mathrm{H}$ & $152,736.800$ & $578,200.976$ & SURV & GPS deriv. & ERC\#044041 & Tube to shore \\
\hline P3007 & TDP-15A-S & 1.0 & HR3D & $152,561.596$ & $573,691.908$ & SURV & GPS deriv. & ERC\#044041 & Tube to shore \\
\hline P3008 & TDP-15A-M & 1.5 & HR3D & $152,561.596$ & $573,691.908$ & SURV & GPS deriv. & ERC\#044041 & Tube to shore \\
\hline P3009 & TDP-15A-D & 2.0 & HR3D & $152,561.596$ & $573,691.908$ & SURV & GPS deriv. & ERC\#044041 & Tube to shore \\
\hline P3010 & TDP-39A-S & 1.0 & HR3D & $151,634.234$ & $572,596.074$ & SURV & GPS deriv. & ERC\#044041 & Tube to shore \\
\hline P3011 & TDP-39A-M & 1.5 & HR3D & $151,634.234$ & $572,596.074$ & SURV & GPS deriv. & ERC\#044041 & Tube to shore \\
\hline P3012 & TDP-39A-D & 2.0 & HR3D & $151,634.234$ & $572,596.074$ & SURV & GPS deriv. & ERC\#044041 & Tube to shore \\
\hline P3013 & TK-14-M & 1.5 & KR4 & $148,482.730$ & $569,963.760$ & EST & ARCmap & ERC\#044041 & Tube to shore; Arc-View loc est. \\
\hline P3014 & TK-14-D & 2.0 & KR4 & $148,482.730$ & $569,963.760$ & EST & ARCmap & ERC\#044041 & Tube to shore; Arc-View loc est. \\
\hline P3015 & TK-14-Dc & 2.0 & KR4 & $148,482.730$ & $569,963.760$ & EST & ARCmap & ERC\#044041 & Tube to shore; Arc-View loc est. \\
\hline P3016 & TK-20-S & 1.0 & KR4 & $148,266.830$ & $569,671.660$ & EST & ARCmap & ERC\#044041 & Tube to shore; Arc-View loc est. \\
\hline P3017 & TK-20-M & 1.5 & KR4 & $148,266.830$ & $569,671.660$ & EST & ARCmap & ERC\#044041 & Tube to shore; Arc-View loc est. \\
\hline
\end{tabular}


Table 6.2. Transect Locator Pins for Riverbed Pore Water Sampling Sites

\begin{tabular}{|c|c|c|c|c|c|}
\hline Segment $^{1}$ & $\begin{array}{l}\text { Transect Pin } \\
\text { Identifier }\end{array}$ & $\begin{array}{c}\text { Transect Pin } \\
\text { Field Label }\end{array}$ & $\begin{array}{c}\text { GPS ID }^{2} \\
\text { (ERC) }\end{array}$ & $\begin{array}{c}\text { Pin } \\
\text { Northing } \\
\text { (m-NAD83) }\end{array}$ & $\begin{array}{c}\text { Pin } \\
\text { Easting } \\
\text { (m-NAD83) }\end{array}$ \\
\hline HR3D & TD-01-PIN & TD-1-PIN & 3046 & $153,303.150$ & $574,052.909$ \\
\hline HR3D & TD-02-PIN & TD-2-PIN & 3045 & $153,248.054$ & $574,027.630$ \\
\hline HR3D & TD-03-PIN & TD-3-PIN & 3044 & $153,194.543$ & $573,998.618$ \\
\hline HR3D & TD-04-PIN & TD-4-PIN & 3043 & $153,136.560$ & $573,980.324$ \\
\hline HR3D & TD-05-PIN & TD-5-PIN & 3042 & $153,080.499$ & $573,957.244$ \\
\hline HR3D & TD-06-PIN & TD-6-PIN & 1062 & $153,021.592$ & $573,942.950$ \\
\hline HR3D & TD-07-PIN & TD-7-PIN & 3040 & $152,962.288$ & $573,930.473$ \\
\hline HR3D & TD-08-PIN & TD-8-PIN & 1060 & $152,905.052$ & $573,909.922$ \\
\hline HR3D & TD-09-PIN & TD-9-PIN & 3037 & $152,846.860$ & $573,892.948$ \\
\hline HR3D & TD-10-PIN & TD-10-PIN & 3036 & $152,788.065$ & $573,878.218$ \\
\hline HR3D & TD-11-PIN & TD-11-PIN & 3035 & $152,740.667$ & $573,839.770$ \\
\hline HR3D & TD-12-PIN & TD-12-PIN & 1055 & $152,684.024$ & $573,817.434$ \\
\hline HR3D & TD-13-PIN & TD-13-PIN & 1054 & $152,631.213$ & $573,787.899$ \\
\hline HR3D & TD-14-PIN & TD-14-PIN & 1053 & $152,591.524$ & $573,743.109$ \\
\hline HR3D & TD-15-PIN & TD-15-PIN & 1051 & $152,551.734$ & $573,696.718$ \\
\hline HR3D & TD-16-PIN & TD-16-PIN & 1047 & $152,522.338$ & $573,644.475$ \\
\hline HR3D & TD-17-PIN & TD-17-PIN & 1043 & $152,483.426$ & $573,597.681$ \\
\hline HR3D & TD-18-PIN & TD-18-PIN & 3000 & $152,439.588$ & $573,555.476$ \\
\hline HR3D & TD-20-PIN & TD-20-PIN & 3003 & $152,359.195$ & $573,459.972$ \\
\hline HR3D & TD-21-PIN & TD-21-PIN & 3004 & $152,325.551$ & $573,409.594$ \\
\hline HR3D & TD-22-PIN & TD-22-PIN & 3007 & $152,298.310$ & $573,355.409$ \\
\hline HR3D & TD-23-PIN & TD-23-PIN & 3009 & $152,270.356$ & $573,301.380$ \\
\hline HR3D & TD-24-PIN & TD-24-PIN & 3010 & $152,237.588$ & $573,250.184$ \\
\hline HR3D & TD-25-PIN & TD-25-PIN & 3011 & $152,205.855$ & $573,198.523$ \\
\hline HR3D & TD-26-PIN & TD-26-PIN & 3012 & $152,169.687$ & $573,149.772$ \\
\hline HR3D & TD-27-PIN & TD-27-PIN & 3015 & $152,131.038$ & $573,102.808$ \\
\hline HR3D & TD-28-PIN & TD-28-PIN & 3016 & $152,096.816$ & $573,052.675$ \\
\hline HR3D & TD-29-PIN & TD-29-PIN & 3017 & $152,058.909$ & $573,005.317$ \\
\hline HR3D & TD-30-PIN & TD-30-PIN & 3018 & $152,017.450$ & $572,960.847$ \\
\hline HR3D & TD-31-PIN & TD-31-PIN & 3019 & $151,967.977$ & $572,925.804$ \\
\hline HR3D & TD-32-PIN & TD-32-PIN & 3021 & $151,909.104$ & $572,909.054$ \\
\hline HR3D & TD-33-PIN & TD-33-PIN & 3022 & $151,861.967$ & $572,870.749$ \\
\hline HR3D & TD-34-PIN & TD-34-PIN & 3023 & $151,824.685$ & $572,822.662$ \\
\hline HR3D & TD-38-PIN & TD-38-PIN & 1040 & $151,680.139$ & $572,636.203$ \\
\hline HR3D & TD-39-PIN & TD-39-PIN & 1008 & $151,627.416$ & $572,605.811$ \\
\hline HR3D & TD-41-PIN & TD-41-PIN & 1010 & $151,530.342$ & $572,532.445$ \\
\hline HR3D & TD-42-PIN & TD-42-PIN & 1016 & $151,491.464$ & $572,486.073$ \\
\hline HR3D & TD-43-PIN & TD-43-PIN & 1020 & $151,441.942$ & $572,450.693$ \\
\hline
\end{tabular}


Table 6.2. (contd)

\begin{tabular}{|c|c|c|c|c|c|}
\hline Segment $^{1}$ & $\begin{array}{l}\text { Transect Pin } \\
\text { Identifier }\end{array}$ & $\begin{array}{c}\text { Transect Pin } \\
\text { Field Label }\end{array}$ & $\begin{array}{c}\text { GPS ID }^{2} \\
\text { (ERC) }\end{array}$ & $\begin{array}{c}\text { Pin } \\
\text { Northing } \\
\text { (m-NAD83) }\end{array}$ & $\begin{array}{c}\text { Pin } \\
\text { Easting } \\
\text { (m-NAD83) }\end{array}$ \\
\hline HR3D & TD-44-PIN & TD-44-PIN & 1023 & $151,395.878$ & $572,410.771$ \\
\hline HR3D & TD-45-PIN & TD-45-PIN & 1024 & $151,355.697$ & $572,365.300$ \\
\hline HR3D & TD-46-PIN & TD-46-PIN & 1025 & $151,310.244$ & $572,325.814$ \\
\hline HR3D & TD-47-PIN & TD-47-PIN & 1026 & $151,264.535$ & $572,285.435$ \\
\hline HR3D & TD-48-PIN & TD-48-PIN & 1027 & $151,214.928$ & $572,250.221$ \\
\hline HR3D & TD-49-PIN & TD-49-PIN & 1032 & $151,170.266$ & $572,210.631$ \\
\hline HR3D & TD-50-PIN & TD-50-PIN & 1037 & $151,127.487$ & $572,167.577$ \\
\hline HR3D & TD-51-PIN & TD-51-PIN & 1039 & $151,077.304$ & $572,133.504$ \\
\hline HR3D & TD-52-PIN & TD-52-PIN & 1038 & $151,032.393$ & $572,093.526$ \\
\hline $\mathrm{HR} 3 \mathrm{H}$ & TH-01-PIN & TH-1-PIN & 2009 & $152,721.560$ & $578,227.373$ \\
\hline $\mathrm{HR} 3 \mathrm{H}$ & TH-01A-PIN & TH-DS-6-PIN & 2007 & $152,718.181$ & $578,216.364$ \\
\hline $\mathrm{HR} 3 \mathrm{H}$ & TH-02-PIN & TH-2-PIN & 2013 & $152,774.272$ & $578,195.167$ \\
\hline $\mathrm{HR} 3 \mathrm{H}$ & TH-03-PIN & TH-3-PIN & 2014 & $152,838.268$ & $578,184.741$ \\
\hline $\mathrm{HR} 3 \mathrm{H}$ & TH-04-PIN & TH-4-PIN & 2015 & $152,898.669$ & $578,160.292$ \\
\hline $\mathrm{HR} 3 \mathrm{H}$ & TH-05-PIN & TH-5-PIN & 2016 & $152,961.721$ & $578,130.453$ \\
\hline $\mathrm{HR} 3 \mathrm{H}$ & TH-06-PIN & TH-6-PIN & 2017 & $153,012.770$ & $578,097.083$ \\
\hline $\mathrm{HR} 3 \mathrm{H}$ & TH-07-PIN & TH-7-PIN & 2019 & $153,061.548$ & $578,067.972$ \\
\hline $\mathrm{HR} 3 \mathrm{H}$ & TH-08-PIN & TH-8-PIN & 2020 & $153,111.026$ & $578,032.774$ \\
\hline $\mathrm{HR} 3 \mathrm{H}$ & TH-09-PIN & TH-9-PIN & 2021 & $153,162.767$ & $577,993.254$ \\
\hline $\mathrm{HR} 3 \mathrm{H}$ & TH-10-PIN & TH-10-PIN & 2025 & $153,207.167$ & $577,955.102$ \\
\hline $\mathrm{HR} 3 \mathrm{H}$ & TH-11-PIN & TH-11-PIN & 2027 & $153,247.786$ & $577,910.338$ \\
\hline $\mathrm{HR} 3 \mathrm{H}$ & TH-12-PIN & TH-12-PIN & 2028 & $153,295.323$ & $577,871.901$ \\
\hline $\mathrm{HR} 3 \mathrm{H}$ & TH-13-PIN & TH-13-PIN & 2029 & $153,340.646$ & $577,829.957$ \\
\hline $\mathrm{HR} 3 \mathrm{H}$ & TH-14-PIN & TH-14-PIN & 2030 & $153,377.879$ & $577,788.695$ \\
\hline $\mathrm{HR} 3 \mathrm{H}$ & TH-15-PIN & TH-15-PIN & 2033 & $153,412.804$ & $577,737.405$ \\
\hline $\mathrm{HR} 3 \mathrm{H}$ & TH-16-PIN & TH-16-PIN & 2034 & $153,451.180$ & $577,681.417$ \\
\hline $\mathrm{HR} 3 \mathrm{H}$ & TH-17-PIN & TH-17-PIN & 2035 & $153,478.763$ & $577,642.411$ \\
\hline $\mathrm{HR} 3 \mathrm{H}$ & TH-18-PIN & TH-DS-5-PIN & 2005 & $152,669.622$ & $578,252.554$ \\
\hline $\mathrm{HR} 3 \mathrm{H}$ & TH-19-PIN & TH-DS-4-PIN & 2004 & $152,621.806$ & $578,290.208$ \\
\hline $\mathrm{HR} 3 \mathrm{H}$ & TH-20-PIN & TH-DS-3-PIN & 2003 & $152,577.402$ & $578,332.138$ \\
\hline $\mathrm{HR} 3 \mathrm{H}$ & TH-21-PIN & TH-DS-2-PIN & 2002 & $152,539.336$ & $578,379.518$ \\
\hline $\mathrm{HR} 3 \mathrm{H}$ & TH-22-PIN & TH-DS-1-PIN & 2001 & $152,499.373$ & $578,425.129$ \\
\hline
\end{tabular}





\subsection{Columbia River Water Sampling Locations}

The SESP collects water samples from the Columbia River at variety of locations (Figure 7.1). The sampling schedules and results are described in annual reports (e.g., Bisping 2009 and Poston et al. 2009, respectively). These locations include:

- Fixed-location monitoring stations at Priest Rapids Dam and the Richland pumphouse.

- Transects across the Columbia River at Vernita Bridge, 100-N Area, the Hanford town site, 300 Area, and Richland pumphouse.

- Near-shore sites along the shorelines at Vernita Bridge, 100-N Area, 100-F Area, Hanford town site, 300 Area, and Richland pumphouse.

The sampling sites at Priest Rapids Dam and Vernita Bridge are upstream from Hanford Site facilities and therefore provide reference data from locations unaffected by releases from the Hanford Site.

Samples collected from all other locations are used to identify impacts caused by past releases associated with the Hanford Site reactor operating years (1944 to 1987). The city of Richland pumphouse is nearest location downstream of Hanford from which Columbia River water is withdrawn to provide water for the Richland municipal drinking system.

The fixed-location monitoring stations at Priest Rapids Dam and the Richland pumphouse consist of both an automated sampler and a continuous flow system. Transect sampling, i.e., multiple samples collected along a line across the Columbia River, was initiated as a result of a special study conducted during 1987 and 1988 (Dirkes et al. 1993). The study concluded that, under certain flow conditions, contaminants entering the river via release from the Hanford Site are not completely mixed with river water by the time the contaminants reach the routine monitoring stations located downriver. Incomplete mixing results in a slightly conservative (high) bias in the data generated using the routine, single-point, sampling system at the Richland pumphouse. During 1999, transect sampling strategy was modified, with some of the mid-river sampling points shifted to near-shore locations in the vicinity of the transect. For example, at the 100-N Area, instead of collecting ten evenly-spaced cross-river transect samples, only six cross-river samples were collected, and the other four samples were obtained at near-shore locations. This sampling pattern has been used since 1999 and allows for the cross-river concentration profile to be determined and also provides information over a larger portion of the Hanford shoreline where the highest contaminant concentrations are expected.

Transect and near-shore samples at Vernita Bridge and the Richland pumphouse are collected quarterly, while at 100-N Area, Hanford town site, and 300 Area they are collected annually during late summer when river flows are low (Bisping 2009). The river sampling locations and the methods used for sample collection are discussed in detail in DOE-RL (2008).

\subsection{Columbia River Water Sampling Locations and Coordinates}

The name and geographic coordinates for the Columbia River water sampling locations are listed in Table 7.1. The location names are based on usage in the HEIS database. The fixed sampling location at 
Priest Rapids Dam is simply referred to as "Priest Rapids Dam” because the sampler is located inside the dam and collects samples from a raw water feed line. Priest Rapids Dam is located approximately 9.5 miles upstream from the Vernita Bridge. All other location names utilize a geographic descriptive name and the HRM system to identify the river location. For example, "Rich.Pmph HRM 46.4" indicates the Richland pumphouse at a location 4 tenths of the downstream distance between HRM signposts \#46 and \#47.

The cross-river transect locations (multiple samples collected on a cross-river transect) are identified by a geographic descriptive name, the Hanford River Marker numbering system, and a transect station number (typically 1-4 or 1-10). The transect station numbers represent roughly equally spaced sampling locations, with the lowest station number occurring on the Hanford shoreline. For example, 300 Area-1 HRM 43.1 signifies a transect sample collected at the 300 Area at HRM 43.1 at transect station number 1 (closest to the Hanford Site shoreline). A second example is 300 Area-5 HRM 43.1, signifying a transect station at the 300 Area at HRM 43.1 at transect station 5, which is approximately halfway across the river. Because the transect and nearshore river water samples are collected by boat, the exact transect locations vary somewhat with each sampling event. Only the station coordinates from the Hanford shoreline (right bank or station \#1) and the opposite shore (left bank or station \#10) are listed in Table 7.1. Detailed maps and description of transect sampling locations are provided in the SESP Locations Manual (PNNL controlled document, SESP-SLM-01).

Coordinates for the river sampling locations described in Table 7.1 are provided in Washington State Plane (South Zone), coordinate systems; units are meters; North American Datum of 1983. Coordinates were obtained using GPS equipment.

\subsection{River Stage Recorders}

The elevation of the Columbia River stage is recorded hourly at stations positioned at each of the former reactor areas and the 300 Area (Table 7.2). The stations are typically located at the river water intake structures for the former reactor areas, and at a water intake structure at the 300 Area. The stage recording project is currently managed by CHPRC as part of the Soil and Groundwater Remediation Project. Data are available for viewing and downloading using the Virtual Library system. Coordinates listed are in the Washington State Plan (South Zone) system; units are meters; North American Datum of 1983. 


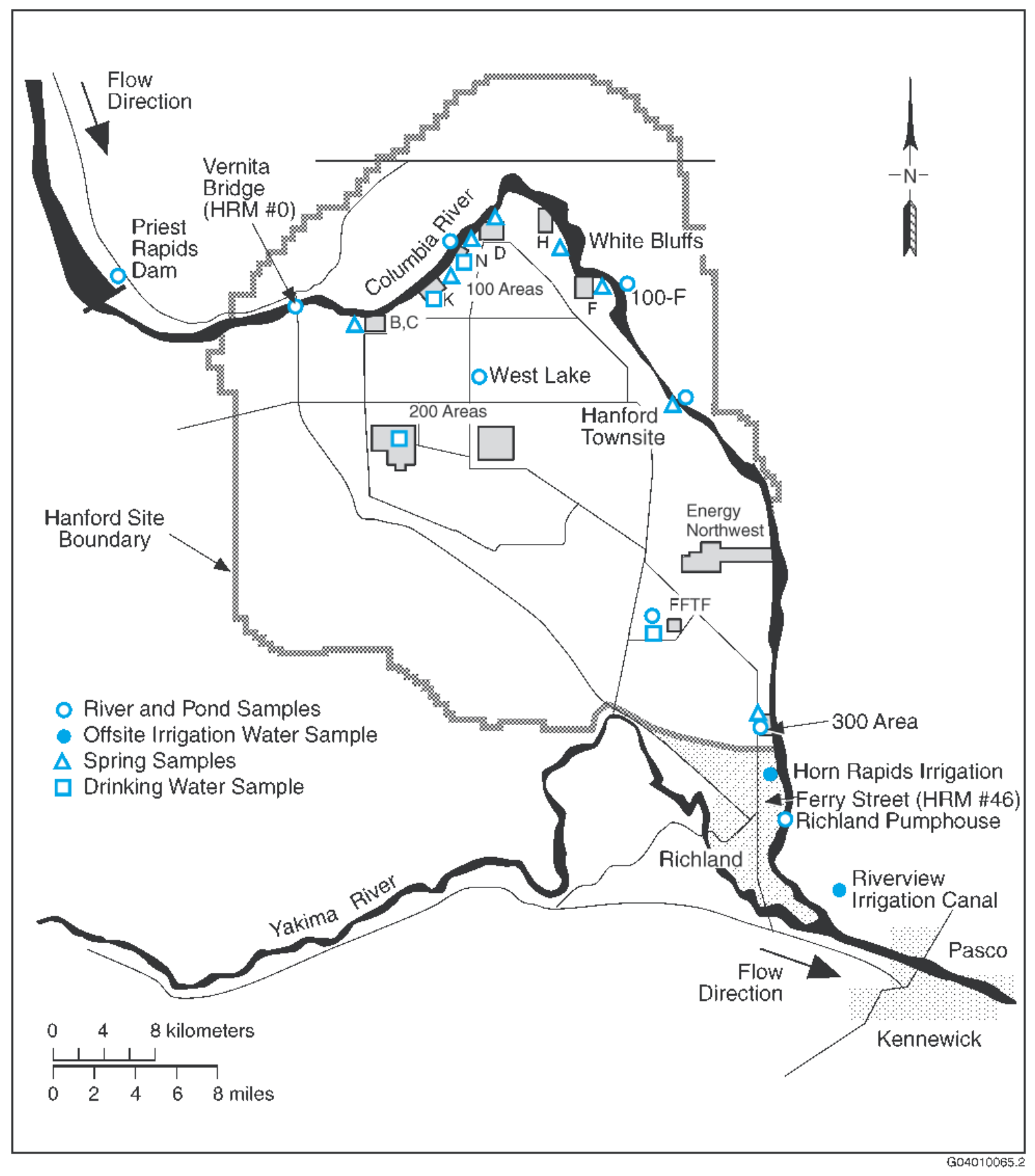

Figure 7.1. Index Map for Columbia River Water Sampling Sites 
Table 7.1. Columbia River Water Sampling Locations in the Vicinity of the Hanford Site

\begin{tabular}{|c|c|c|c|c|}
\hline Geographic Region Along Columbia River & $\begin{array}{l}\text { River Water Sampling Location } \\
\qquad \text { Name }^{1}\end{array}$ & Sample Type ${ }^{2}$ & $\begin{array}{l}\text { Northing } \\
\text { (m-NAD83) }\end{array}$ & $\begin{array}{c}\text { Easting } \\
\text { (m-NAD83) }\end{array}$ \\
\hline Priest Rapids Dam & Priest Rapids-River & Fixed Location & $146,056.722$ & $545,351.336$ \\
\hline Richland Pumphouse & Rich.Pmphs HRM 46.4 & Fixed Location & $109,812.049$ & $595,441.466$ \\
\hline Vernita Bridge, Hanford Site Shore & Vernita-1 HRM 0.3 & Transect & $145,808.019$ & $558,996.328$ \\
\hline Vernita Bridge, Grant Co. Shore & Vernita-4 HRM 0.3 & Transect & $146,018.380$ & $558,910.056$ \\
\hline 100-N Area & 100 N Shore HRM 8.4 & Nearshore & $149,044.428$ & $570,527.283$ \\
\hline 100-N Area & 100 N Shore HRM 8.9 & Nearshore & $149,930.777$ & $571,220.846$ \\
\hline 100-N Area & 100 N Shore HRM 9.2 & Nearshore & $150,222.208$ & $571,423.982$ \\
\hline 100-N Area, Hanford Site Shore & 100 N-1 HRM 9.5 & Transect & $150,535.875$ & $571,626.834$ \\
\hline 100-N Area, Grant Co. Shore & 100 N-10 HRM 9.5 & Transect & $150,754.451$ & $571,310.576$ \\
\hline 100-N Area & 100 N Shore HRM 9.8 & Nearshore & $151,018.280$ & $571,995.955$ \\
\hline 100-F Area & 100 F Shore HRM 18 & Nearshore & $149,049.329$ & $579,832.753$ \\
\hline 100-F Area, Hanford Site Shore & 100 F-1 HRM 19.0 & Transect & $109,850.604$ & $595,772.085$ \\
\hline 100-F Area, Franklin Co. Shore & 100 F-10 HRM 19.0 & Transect & $148,447.371$ & $581,371.414$ \\
\hline 100-F Area & 100 F Shore HRM 22 & Nearshore & $144,147.523$ & $583,167.976$ \\
\hline 100-F Area & 100 F Shore HRM 23 & Nearshore & $143,272.758$ & $583,417.486$ \\
\hline Hanford Town Site & Hanfrd Twnsite HRM 26 & Nearshore & $139,249.682$ & $586,654.140$ \\
\hline Hanford Town Site & Hanfrd Twnsite HRM 27 & Nearshore & $138,366.365$ & $587,839.777$ \\
\hline Hanford Town Site & Hanfrd Twnsite HRM 28 & Nearshore & $137,623.593$ & $586,439.761$ \\
\hline Hanford Town Site, Hanford Site Shore & Hanfrd TS-1 HRM 28.7 & Transect & $137,085.683$ & $589,951.799$ \\
\hline Hanford Town Site, Franklin Co. Shore & Hanfrd TS-10 HRM 28.7 & Transect & $137,277.023$ & $590,109.980$ \\
\hline Hanford Town Site & Hanfrd Twnsite HRM 30 & Nearshore & $135,433.944$ & $591,004.194$ \\
\hline 300 Area & 300 Area Sr HRM 41.5 & Nearshore & $117,518.980$ & $594,227.923$ \\
\hline 300 Area & 300 Area Sr HRM 42.1 & Nearshore & $116,686.645$ & $594,317.796$ \\
\hline
\end{tabular}


Table 7.1. (contd)

\begin{tabular}{|c|c|c|c|c|}
\hline Geographic Region Along Columbia River & $\begin{array}{l}\text { River Water Sampling Location } \\
\text { Name }^{1}\end{array}$ & Sample Type ${ }^{2}$ & $\begin{array}{l}\text { Northing } \\
\text { (m-NAD83) }\end{array}$ & $\begin{array}{l}\text { Easting } \\
\text { (m-NAD83) }\end{array}$ \\
\hline 300 Area & 300 Area Sr HRM 42.5 & Nearshore & $116,055.079$ & $594,450.735$ \\
\hline 300 Area & 300 Area Sr HRM 42.9 & Nearshore & $115,224.428$ & $594,648.372$ \\
\hline 300 Area, Hanford Site Shore & 300 Area-1 HRM 43.1 & Transect & $114,870.592$ & $594,769.339$ \\
\hline 300 Area, Franklin Co. Shore & 300 Area-10 HRM 43.1 & Transect & $114,880.240$ & $595,384.911$ \\
\hline Richland & Rich.Pmphs HRM 43.5 & Nearshore & $114,370.836$ & $594,800.236$ \\
\hline Richland & Rich.Pmphs HRM 43.9 & Nearshore & $113,704.977$ & $594,872.221$ \\
\hline Richland & Rich.Pmphs HRM 45.0 & Nearshore & $112,098.196$ & $595,197.638$ \\
\hline Richland & Rich.Pmphs HRM 45.8 & Nearshore & $110,789.565$ & $595,395.298$ \\
\hline Richland Pumphouse, Benton Co. Shore & Rich.Pmphs-1 HRM 46.4 & Transect & $109,846.359$ & $595,502.550$ \\
\hline Richland Pumphouse, Franklin Co. Shore & Rich.Pmphs-10 HRM 46.4 & Transect & $109,936.193$ & $596,263.717$ \\
\hline
\end{tabular}


Table 7.2. River Stage Recorder Locations Along the Hanford Reach

\begin{tabular}{|c|c|c|c|c|c|}
\hline Location & $\begin{array}{c}\text { River Stage } \\
\text { Recorder } \\
\text { Name }\end{array}$ & Start Date & End Date & $\begin{array}{c}\text { Northing } \\
\text { (m-NAD88) }\end{array}$ & $\begin{array}{c}\text { Easting } \\
\text { (m-NAD88) }\end{array}$ \\
\hline 100-B Area & B-River & 11-Mar-92 & Operating & $145,242.190$ & $564,832.286$ \\
\hline 100-K Area & K-River & 25-Mar-08 & Operating & $147,084.250$ & $568,758.160$ \\
\hline 100-N Area & N-River & 26-Nov-94 & Operating & $149,457.100$ & $570,988.731$ \\
\hline 100-D Area & D-River & 11-Nov-96 & Operating & $151,738.440$ & $572,778.400$ \\
\hline 100-H Area & H-River & 27-Sep-91 & Operating & $153,492.956$ & $577,547.540$ \\
\hline 100-F Area & F-River & 18-Sep-92 & Operating $^{1}$ & $148,139.838$ & $580,977.001$ \\
\hline 300 Area & SWS-1 & 06-Nov-91 & 01-May-97² & $115,780.554$ & $594,582.972$ \\
\hline 300 Area & 3-River & 01-Sep-97 & Operating & $115,780.554$ & $594,582.972$ \\
\hline
\end{tabular}




\subsection{Proposed Naming Convention for Shoreline Water Quality Monitoring Sites}

Water samples are collected from a variety of sites near the Columbia River, including near-river monitoring wells; riverbank springs; aquifer sampling tubes located along the low stage shoreline; and pore water samples from riverbed sediment. Temporary site names are often assigned to these locations when they are first used. Once established, a unique site identifier is assigned to some sites/facilities for data management purposes, and a descriptive name is developed for use in reports. Unfortunately, no standardized convention has been developed for the latter, resulting in a variety of descriptive names for the same site being incorporated into databases and published reports. This creates a special problem for those sites for which a unique identifier has not been assigned.

\subsection{Site Identifiers and Descriptive Site Names}

The following discussion assumes that:

- Sample collection locations (“sites”) can be uniquely defined geographically (i.e., by survey coordinates). A unique identifier is assigned to each site for data management purposes.

- Sampling sites also need a descriptive name, which is used in interpretive reports and presentations. Descriptive names are for the convenience of the data user and are included as supplemental information in database records.

Currently, unique site identifiers are only assigned to engineered structures that fall under the purview of WAC 173-160 “Minimum Standards for Construction and Maintenance of Wells.” These include groundwater monitoring wells and aquifer sampling tubes. Riverbank springs and other water sampling sites associated with the groundwater/river interface have been assigned site identifiers for data management purposes, but in some instances they are not unique (e.g., riverbank springs). To qualify as a sampling "site," the location must be describable in terms of geographic coordinates (e.g., an XY coordinate as a minimum, with or without a radius of uncertainty; a polygon described by points with coordinates). The following proposal includes a new provision for assigning a unique site identifier to riverbank springs that consistently provide water samples for environmental monitoring. This site identifier is analogous to the existing identifier used for wells (i.e., the WELL_ID in the HEIS database).

Descriptive names for sampling locations should provide some information as to the nature of the site, as well as its geographic location. The primary purpose for the descriptive name is for the convenience of the data user, and not for internal data management purposes - the latter is the role of the unique site identifier. Whenever the need arises to assign names to new sampling locations, it is desirable to retain as much commonality as possible with descriptive names used in previously published reports and long-term historical conventions. But there is no compelling need to revise previously used descriptive names for sampling sites. The scheme proposed in the following paragraphs is suggested for newly discovered, established, or equipped sampling sites. 


\subsection{Proposed Convention for Descriptive Site Names}

The naming convention proposed below attempts to honor the following characteristics for assigning a descriptive name to a sampling site (...to be used in conjunction with a unique site identification number):

- An indication of the nature of the sampling facility or site

- Some form of geographic orientation

- Similarity to previously established naming conventions

These characteristics have been honored in the groundwater well-naming convention that has existed on the Hanford Site since 1956 (Chamness and Merz 1993). For example, the descriptive name for monitoring well 199-K-30 in the 100-K Area is decoded as follows: "199-“ indicates a groundwater monitoring well in the 100 Area; "K-“ indicates the $100-\mathrm{K}$ Area; and " 30 ” is a sequential number for wells installed in the 100-K Area. The well also has a unique identifier (A4655) that is used for data management purposes.

For other types of water quality sampling sites located along the river shoreline, coded letters can be used to indicate the type of site, followed by a second code that refers to the geographic segment or area, followed by a numeric code that identifies the location between two adjacent Hanford River Marker posts. Suffixes can be added to further describe supplemental information about the site. A summary of the codes proposed for river-related water quality monitoring sites is shown in Table 8.1.

\subsection{Examples of Proposed Site Names}

\section{Riverbank Spring}

- SB-0373 - Riverbank spring along the 100-B Area located at HRM 3.73, i.e., 0.73 of the distance from HRM signpost \#3 downstream toward signpost \#4. Previously used names for this spring are 037-1, SB-037-1, and 100-B Spring.

- S3-4211 - Riverbank spring along the 300 Area located at HRM 42.105. Previously used names for this spring include Spring 7, 42-2, and 300 Area Spring 42-2.

\section{Aquifer Sampling Tube}

- AB-0389-D - Aquifer sampling tube along the 100-B Area shoreline located at HRM 3.89. The suffix “-D” indicates the deepest of three tubes at this site. Previously used name for this tube is 05-D.

- AD-1021-S - Aquifer sampling tube along the 100-D Area shoreline located at HRM 10.21. The suffix "-S" indicates the shallowest of three tubes at this site. Previous name for this tube is DD-39-1. 
Table 8.1. Code for Naming Water Quality Sampling Sites Along the Hanford Reach

Format for Descriptive Name: 12-3333-4

1. Type of water quality sampling site:

$\circ \quad \mathrm{S}=$ Riverbank spring

- $\quad \mathrm{A}=$ Aquifer sampling tube (sample port $>1$ meter bgs) ${ }^{1}$

$\circ \quad \mathrm{P}=$ Riverbed sediment pore water tube (sample port $<1$ meter bgs) ${ }^{1}$

○ $\quad \mathrm{R}=$ Nearshore river water

2. Hanford Site geographic region along Hanford Reach:

- $\quad \mathrm{B}=100-\mathrm{B}$ Area and adjacent upstream/downstream shorelines

- $\mathrm{K}=100-\mathrm{K}$ Area and adjacent upstream/downstream shorelines

- $\quad \mathrm{N}=100-\mathrm{N}$ Area and adjacent upstream/downstream shorelines

- $\quad \mathrm{D}=100$-D Area and adjacent upstream/downstream shorelines

- $\quad \mathrm{H}=100-\mathrm{H}$ Area and adjacent upstream/downstream shorelines

- $\quad \mathrm{F}=100-\mathrm{F}$ Area and adjacent upstream/downstream shorelines

- $\mathrm{T}=$ Hanford town site and adjacent upstream/downstream shorelines

- $3=300$ Area and adjacent upstream/downstream shorelines

- $6=600$ Area (intervening segments, including 200-PO-1 Operable Unit plume)

3. Hanford River Marker system position:

- Measured to the nearest hundredth of a subdivision between signposts

- Coordinates for HRM signposts, and for subdivisions between signposts, are maintained on

1:10,000 scale base maps for the shoreline as ArcMap coverages

4. Optional codes as suffix:

- Sequential site at same approximate location (e.g., multiple springs)

- Shallow, medium, deep indicators (e.g., aquifer tubes; pore water tubes)

- Numeric value for depth below ground surface

- Alpha or numeric value to indicate distance offshore

1. The one-meter value is an arbitrary depth chosen to categorize samples as either surface water (SW) or groundwater (GW) in the media code field of HEIS. The hyporheic zone of the Hanford Reach channel is assumed to be primarily contained within this depth interval. If several sampling ports are installed at the same site, the deepest port is used to determine the category.

- A3-4239-A410cm - Aquifer sampling tube along the 300 Area located at HRM 42.39. The suffix “A $410 \mathrm{~cm}$ " indicates the first of several locations extending offshore (A), and the $410 \mathrm{~cm}$ indicates the depth of the sampling port.

\section{Riverbed Sediment Pore Water}

- PH-1530-A45cm - Riverbed pore water sample tube in the 100-H Area located at HRM 15.30. The sampling site is the first of two that extend in an offshore direction, and the sample port depth is 45 cm bgs. Original name: TH-1A in Hope and Peterson (1996a).

\section{Nearshore River Water}

- R3-4211-SHR - River water sample collected at the 300 Area shore at HRM 42.105. The suffix indicates nearshore river water. Previous name for this sample site is "300 AREA SHR HRM 42.2" (Patton et al. 2002). 



\subsection{Path Forward}

The inventory of water quality monitoring sites in this report has been assembled over a period of many years. While every effort has been made to achieve accuracy in the location and name information, inaccuracies and errors may be present. Use of the tables for future projects involving the Columbia River will likely help reveal those deficiencies, and future versions of the document will incorporate those discoveries. However, the inventory as it stands can be used to update sampling site location information contained in the HEIS. Because numerous historical records for analytical results may contain ambiguous sample site information, it has not been possible to develop a convenient user interface for accessing historical data for certain samples assigned a media code for surface water.

Future work should consider the following:

- Correlate existing analytical records in HEIS for riverbank springs with the discreet locations information presented in Table 4.1. Adopting a unique site identifier for riverbank springs, to be used in sample site location tables in HEIS, will help in this regard.

- Revise existing sample site identifiers assigned to surface water media results, i.e., remove multiple identifiers (when they occur) for a single sampling site. This ambiguity is most frequent with analytical results for samples from riverbank springs.

- Convene a users' group to stimulate discussion of descriptive names for sampling sites. The goal is to adopt a convention that is acceptable to the majority of those working in the Columbia River environment and one that can be easily adopted for new sampling sites. 



\subsection{References}

65 FR 114. 2000. Presidential Proclamation 7319, "Establishment of the Hanford Reach National Monument,” Federal Register.

Arntzen, EV, DR Geist, and PE Dresel. 2006. "Effects of fluctuating river flow on groundwater/surface water mixing in the hyporheic zone of a regulated, large cobble bed river." River Research and Applications, 22(8): 937-946.

Bisping LE. 2009. Hanford Site Environmental Surveillance Master Sampling Schedule for Calendar Year 2009. PNNL-18177, January 2009. Pacific Northwest National Laboratory, Richland, Washington.

Brown DJ. 1963. Status of the Groundwater Beneath Hanford Reactor Areas: January 1962 to January 1963. HW-77170, Hanford Atomic Products Operation, General Electric Company, Richland, Washington.

Buske N and L Josephson. 1989. Water and Sediment Reconnaissance of the Hanford Shoreline, Hanford Reach Project, Data Report 4, Fall 1988. SEARCH Technical Services, Davenport, Washington and published by Hanford Education Action League, Spokane, Washington.

CERCLA - Comprehensive Environmental Response, Compensation, and Liability Act. 1980. Public Law 96-150, as amended, 94 Stat. 2767, 42 USC 9601 et seq.

Chamness MA and JK Merz. 1993. Hanford Wells. PNL-8800, Pacific Northwest Laboratory, Richland, Washington.

Connelly MP. 1997. Assessment of the Chromium Plume West of the 100-D/DR Reactors. BHI-00967, prepared by CH2M HILL Hanford, Inc. for Bechtel Hanford, Inc., Richland, Washington.

Dirkes RL. 1990. 1988 Hanford Riverbank Springs Characterization Report. PNL-7500, Pacific Northwest Laboratory, Richland, Washington.

Dirkes, RL, GW Patton, and BL Tiller. 1993. Columbia River Monitoring: Summary of Chemical Monitoring Along Cross Sections at Vernita Bridge and Richland, PNL-8654, Pacific Northwest Laboratory, Richland, Washington.

DOE-RL. 1992. Sampling and Analysis of 100 Area Springs. DOE/RL-92-12, Rev. 1, prepared by IT Corporation for Westinghouse Hanford Company, U.S. Department of Energy, Richland Field Office, Richland, Washington.

DOE-RL. 2008a. Environmental Monitoring Plan, United States Department of Energy, Richland Operations Office. DOE/RL-91-50, Rev. 4, March 2008. U.S. Department of Energy, Richland, Washington.

DOE-RL. 2008b. Remedial Investigation Work Plan for Hanford Site Releases to the Columbia River. DOE/RL-2008-11, Rev. 0, September 2008. U.S. Department of Energy, Richland, Washington. 
Ecology - Washington State Department of Ecology, U.S. Environmental Protection Agency, and U.S. Department of Energy. 1989. Hanford Federal Facility Agreement and Consent Order. Document No. 89-10, as amended, (The Tri-Party Agreement), Olympia, Washington.

Eliason JR. 1969. Mapping of a Reactor Coolant Effluent Ground Disposal Test Using an Infrared Imaging System and Ground Water Potential and Temperature Measurements. BNWL-CC-2058, Battelle Northwest, Richland, Washington.

Friant SL and LC Hulstrom. 1993. Sampling and Analysis of the 300-FF-5 Operable Unit Springs, Nearshore Sediments, and River Water. WHC-SD-EN-TI-125, Rev. 0, prepared by Pacific Northwest Laboratory for Westinghouse Hanford Company, Richland, Washington.

Fritz, BG, DP Mendoza, and TJ Gilmore. 2009. "Development of an Electronic Seepage Chamber for Extended Use in a River.” Ground Water, Vol. 47, No. 1, January-February 2009, pp. 136-140.

Fritz, BG, RD Mackley, EV Arntzen, DP Mendoza, and GW Patton. 2008. Methods for Assessing the Relative Amounts of Groundwater Discharge into the Columbia River and Measurement of Columbia River Gradient at the Hanford Site's 300 Area. PNNL-17836, September 2008. Pacific Northwest National Laboratory, Richland, Washington.

Fritz, BG, RD Mackley, NP Kohn, GW Patton, TJ Gilmore, DP Mendoza, D McFarland, AL Bunn, and EV Arntzen. 2007. Investigation of the Hyporheic Zone at the 300 Area, Hanford Site. PNNL-16805, October 2007. Pacific Northwest National Laboratory, Richland, Washington.

HAPO. 1963. "Volume 3--Description of the 100-B, 100-C, 100-D, 100-DR, 100-F, and 100-H Production Reactor Plants.” In: Hazards Summary Report. HW-74094, Vol. 3, April 1, 1963. Hanford Atomic Products Operation, General Electric Company, Richland, Washington.

Hartman MJ. 2009. Aquifer Tube Optimization Evaluation. SGW-41497, Rev. 0, August 2009, CH2M HILL Plateau Remediation Company, Richland, Washington.

Hartman MJ and RE Peterson. 2003. Aquifer Sampling Tube Results for Fiscal Year 2003. PNNL-14444, Pacific Northwest National Laboratory, Richland, Washington.

Hartman MJ, LF Morasch, and WD Webber (eds.). 2004. Hanford Site Groundwater Monitoring for Fiscal Year 2003. PNNL-14548, Pacific Northwest National Laboratory, Richland, Washington.

Hartman MJ, JA Rediker, and VS Richie. 2009. Hanford Site Groundwater Monitoring for Fiscal Year 2008. DOE/RL-2008-66, Rev. 0, March 2009. Prepared by CH2M HILL Plateau Remediation Company for the U.S. Department of Energy, Richland, Washington.

Hartman MJ, PE Dresel, JW Lindberg, JP McDonald, DR Newcomer, and EC Thornton. 2002. Fiscal Year 2003 Integrated Monitoring Plan for the Hanford Groundwater Monitoring Project. PNNL-14111, Pacific Northwest National Laboratory, Richland, Washington.

Hope SJ and RE Peterson. 1996a. Chromium Concentrations in 100-H Operable Unit Pore Water Within Chinook Salmon Spawning Habitat of the Hanford Reach, Columbia River. BHI-00345, Rev. 0, prepared by CH2M HILL Hanford, Inc. for Bechtel Hanford, Inc., Richland, Washington. 
Hope SJ and RE Peterson. 1996b. Chromium in River Substrate Pore Water and Adjacent Groundwater: 100-D/DR Area, Hanford Site, Washington. BHI-00778, Rev. 0, prepared by CH2M HILL Hanford, Inc. for Bechtel Hanford, Inc., Richland, Washington.

Kasza, GL. 2009. Sampling and Analysis Plan for Aquifer Sampling Tubes. DOE/RL-2000-59, Rev. 1, February 2009. Prepared by Freestone Environmental Services and CH2M HILL Plateau Remediation Company for the U.S. Department of Energy, Richland, Washington.

McCormack WD and JMV Carlile. 1984. Investigation of Groundwater Springs from the Hanford Shoreline of the Columbia River. PNL-5289, Pacific Northwest Laboratory, Richland, Washington.

Olsen KB and S Kucheryavyy. 1995. Determination of Chromium (VI) in Columbia River Pore Water by Adsorptive Stripping Voltametry. July 1995 letter report to the U.S. Department of Energy, Richland, Washington.

Patton GW, SP Van Verst, BL Tiller, EJ Antonio, and TM Poston. 2002. Survey of Radiological and Chemical Contaminants in the Near-Shore Environment at the Hanford Site 300 Area. PNNL-13692, Pacific Northwest National Laboratory, Richland, Washington.

Peterson, RE. 1997. Summary of River Bank Seepage Sampling Event for FY 1997: 100-BC-5, 100-KR-4, and 100-HR-3 Operable Units. Attachment to Interoffice Memorandum, M.H. Sturges to A. J. Knepp, March 7, 1997, Control No. 041996. Prepared by CH2M HILL Hanford, Inc. for Bechtel Hanford, Inc., Richland, Washington. (Available via Hanford Public Information Repositories and Administrative Records, http://www2.hanford.gov/arpir/default.cfm, Accession Number D197268361)

Peterson RE and VG Johnson. 1992. Riverbank Springs of Groundwater Along the Hanford Reach of the Columbia River, Washington. WHC-EP-0609, Westinghouse Hanford Company, Richland, Washington, 44 pp. plus appendices.

Peterson RE, JV Borghese, and DB Erb. 1998. Aquifer Sampling Tube Installation Completion Report: 100 Area and Hanford Townsite Shorelines. BHI-01153, Rev. 0, prepared by CH2M HILL Hanford, Inc. for Bechtel Hanford, Inc., Richland, Washington.

Poston TM, RW Hanf, RL Dirkes, and LF Morasch (eds.). 2003. Hanford Site Environmental Report for Calendar Year 2002. PNNL-14295, Pacific Northwest National Laboratory, Richland, Washington.

Poston TM, JR Duncan, and RL Dirkes (eds.). 2009. Hanford Site Environmental Report for Calendar Year 2008. PNNL-18427, Pacific Northwest National Laboratory, Richland, Washington.

Probasco KM. 1986. Characterization of Radionuclide Concentrations of the N-Springs Along the Columbia River Shoreline. UNI-3866, UNC Nuclear Industries, Richland, Washington.

Raidl RF and GG Kelty. 2004. Fiscal Year 2003 Annual Summary Report for the In Situ Redox Manipulation Operations. DOE/RL-2004-06, Rev. 0, prepared by Fluor Hanford, Inc. for the U.S. Department of Energy, Richland, Washington.

SESP-SLM-01. Environmental Sampling Locations Manual. PNNL Controlled Document. 
WAC 173-160. "Minimum Standards for Construction and Maintenance of Wells." Washington Administrative Code, Olympia, Washington.

Williams MD, VR Vermeul, JE Szecsody, JS Fruchter, and CR Cole. 2000. 100-D Area In Situ Redox Treatability Test for Chromate-Contaminated Groundwater. PNNL-13349, Pacific Northwest National Laboratory, Richland, Washington. 
Appendix A 


\section{Appendix A}

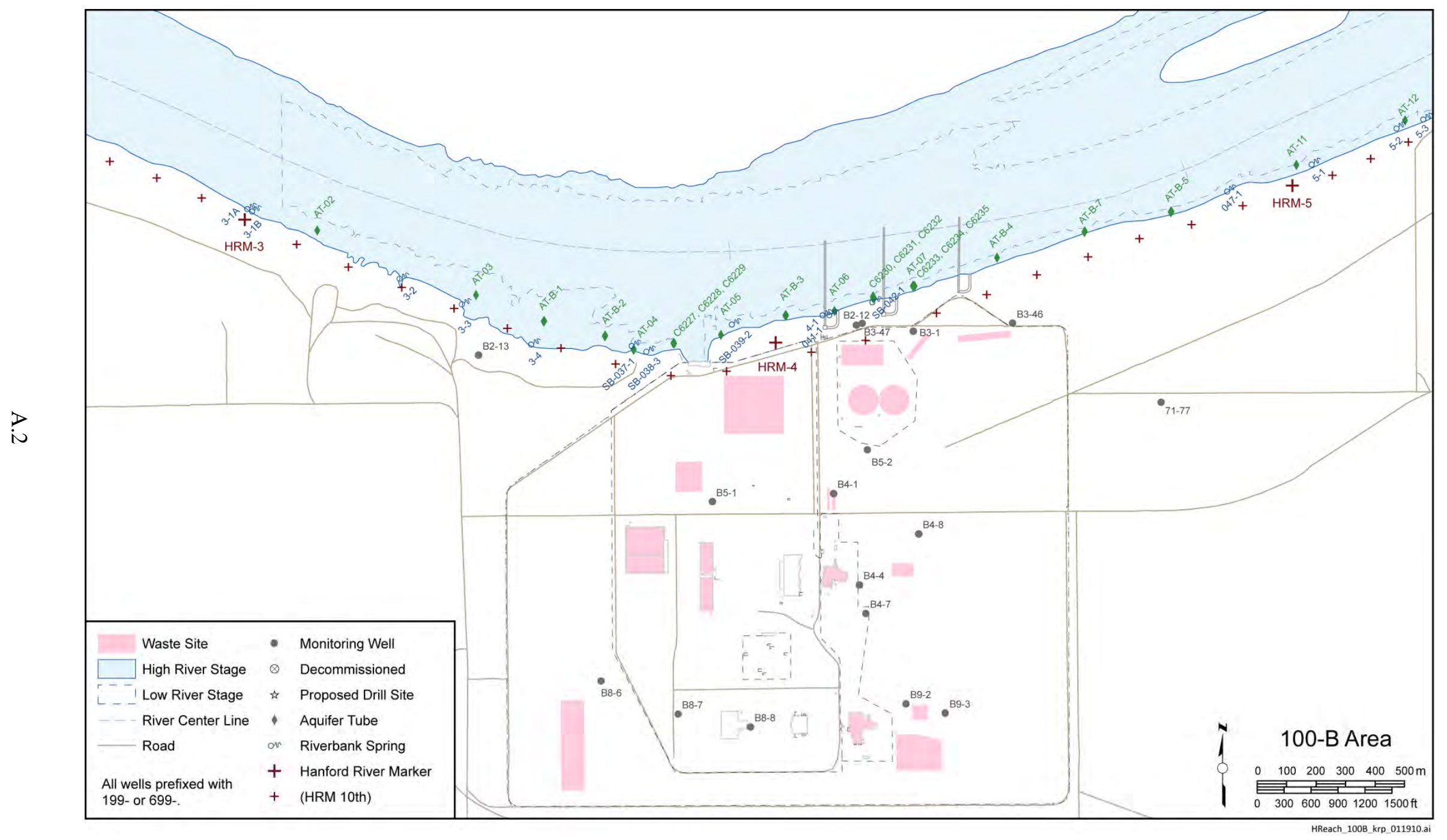

Figure A.1. Water Quality Monitoring Locations Along the 100-B Area Shoreline 


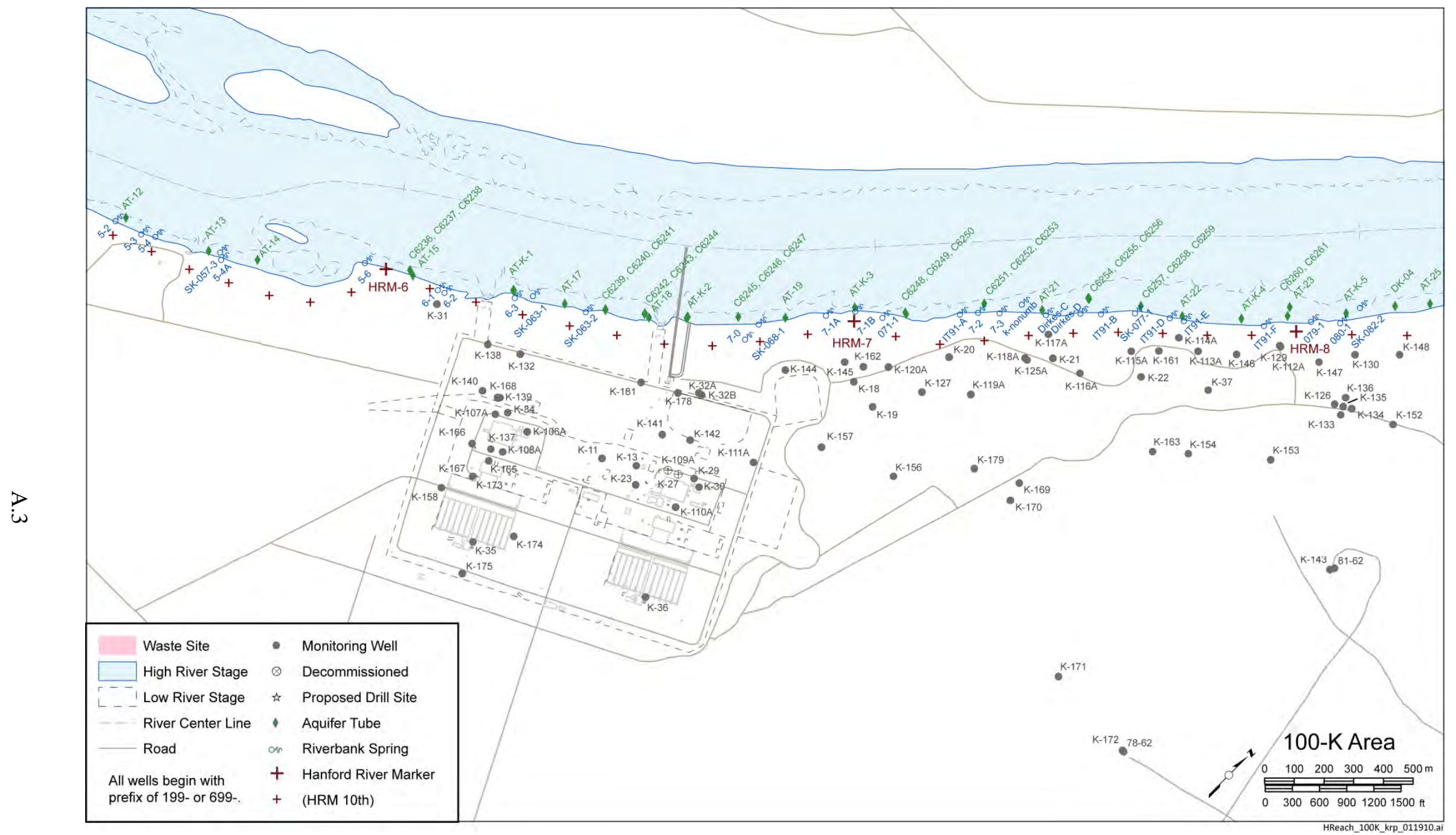

Figure A.2. Water Quality Monitoring Locations Along the 100-K Area Shoreline 


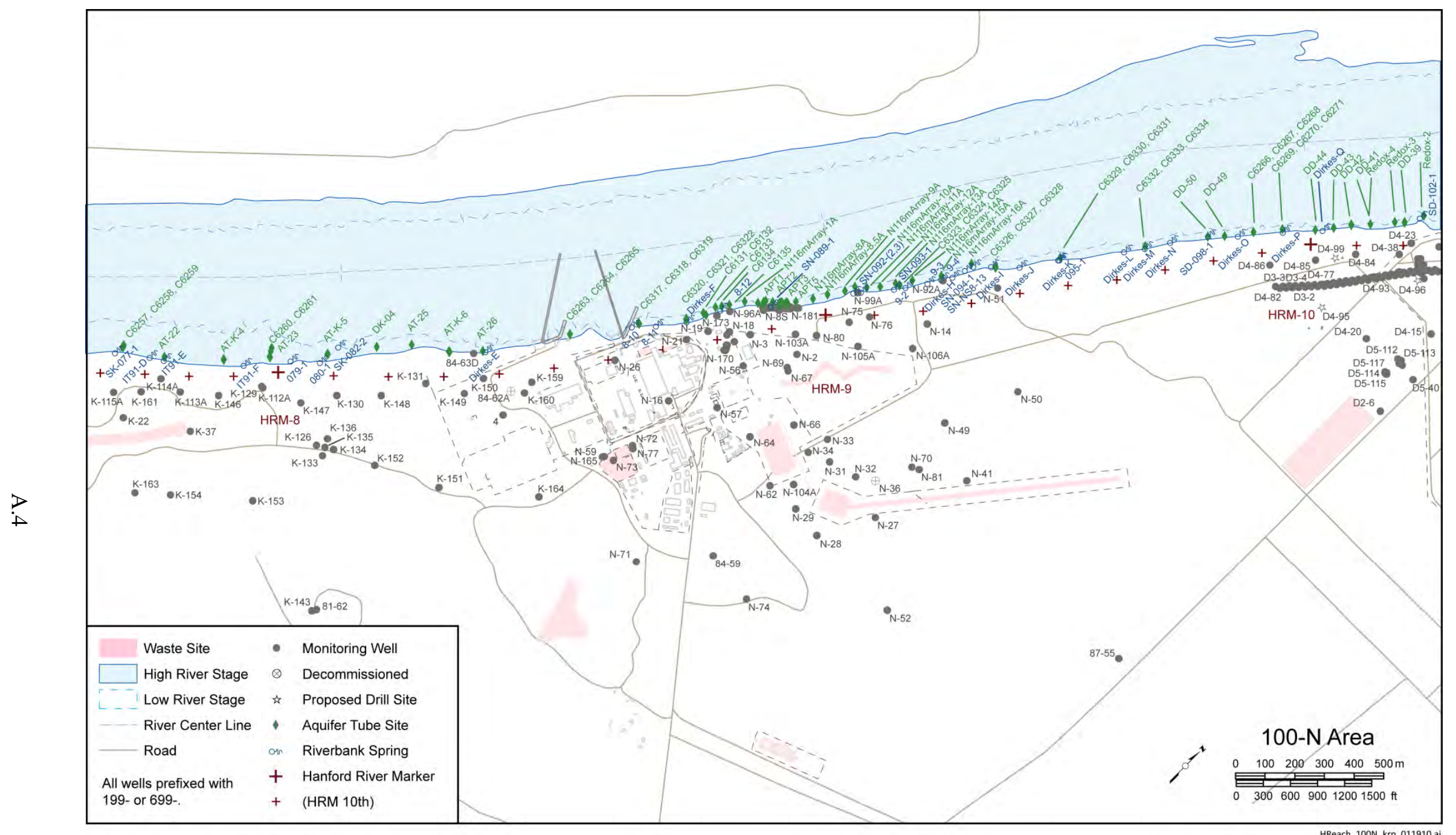

Figure A.3. Water Quality Monitoring Locations Along the 100-N Area Shoreline 


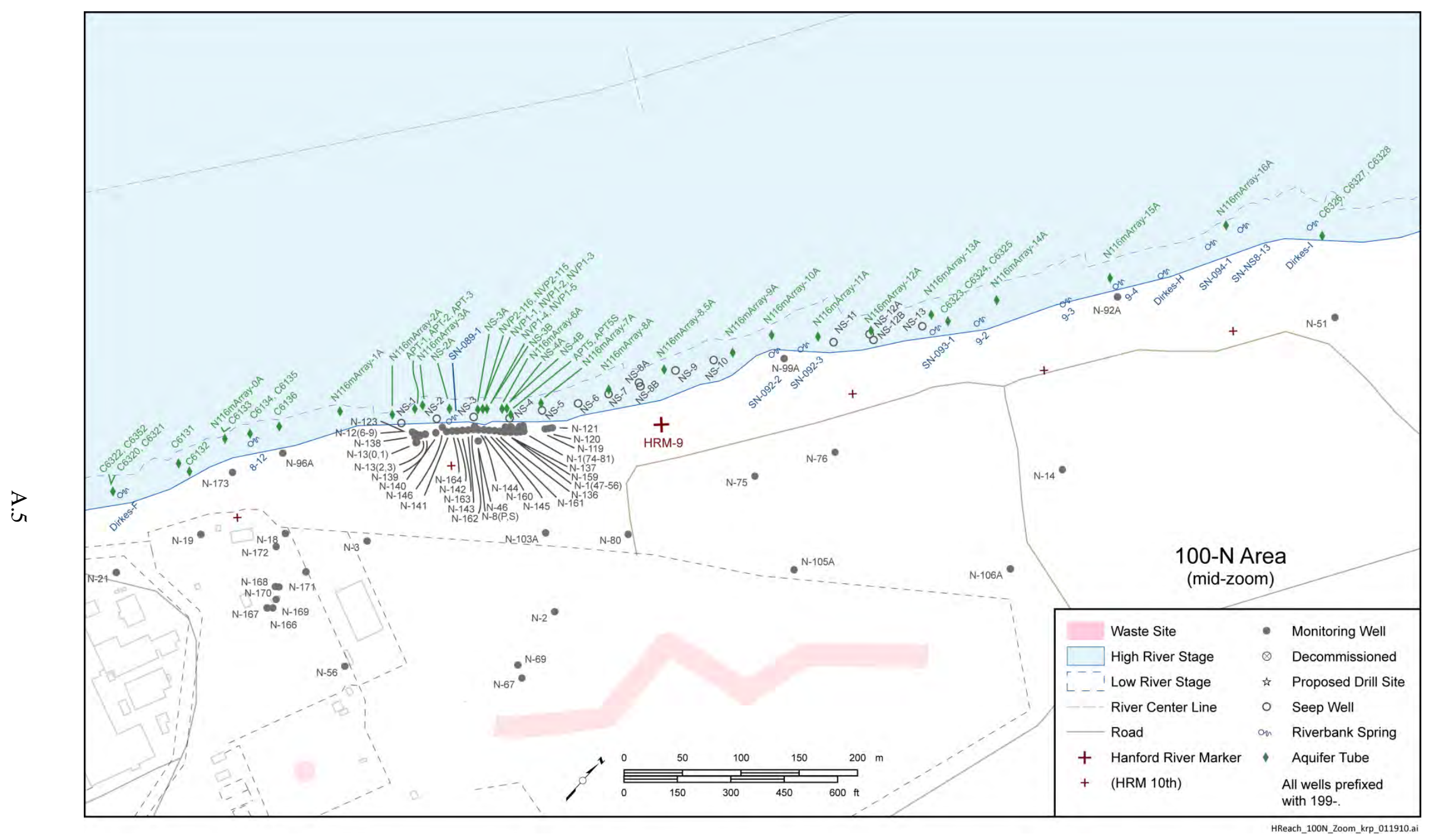

Figure A.4. Water Quality Monitoring Locations Along the Central Portion of the 100-N Area Shoreline 


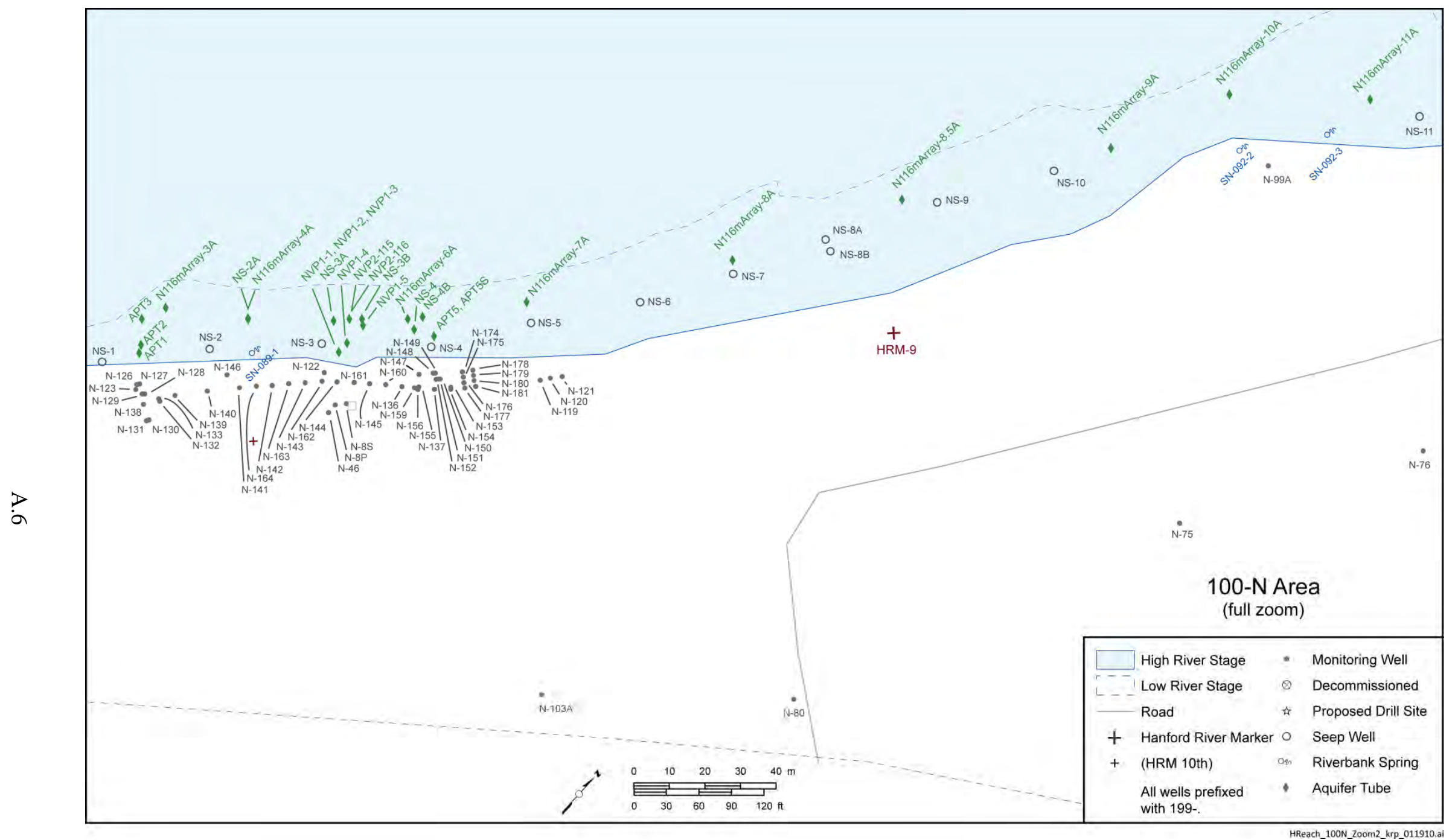

Figure A.5. Water Quality Monitoring Locations Along the Apatite Barrier Portion of the 100-N Area Shoreline 


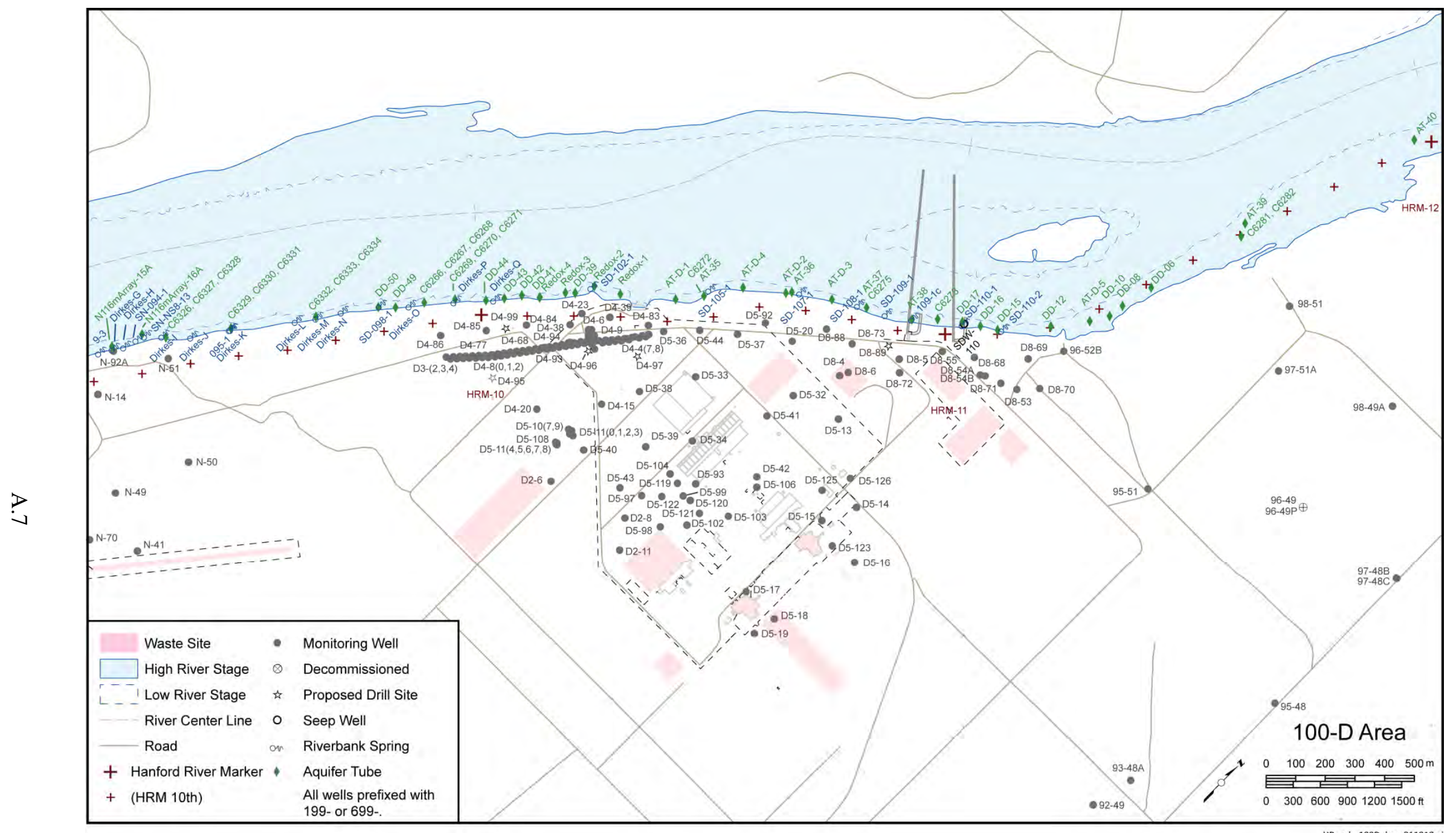

Figure A.6. Water Quality Monitoring Locations Along the 100-D Area Shoreline 


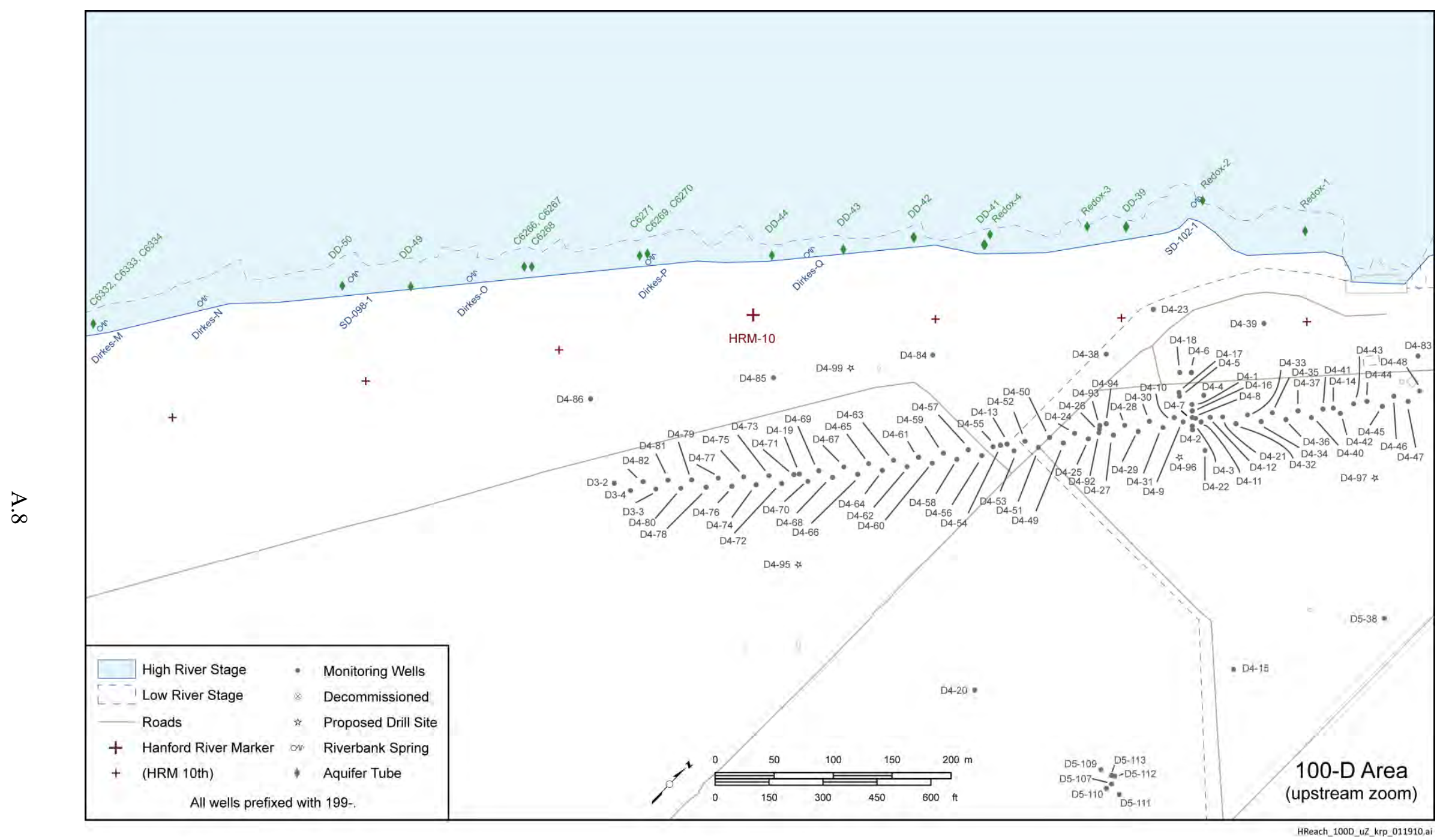

Figure A.7. Water Quality Monitoring Locations Along the ISRM Barrier Portion of the 100-D Area Shoreline 


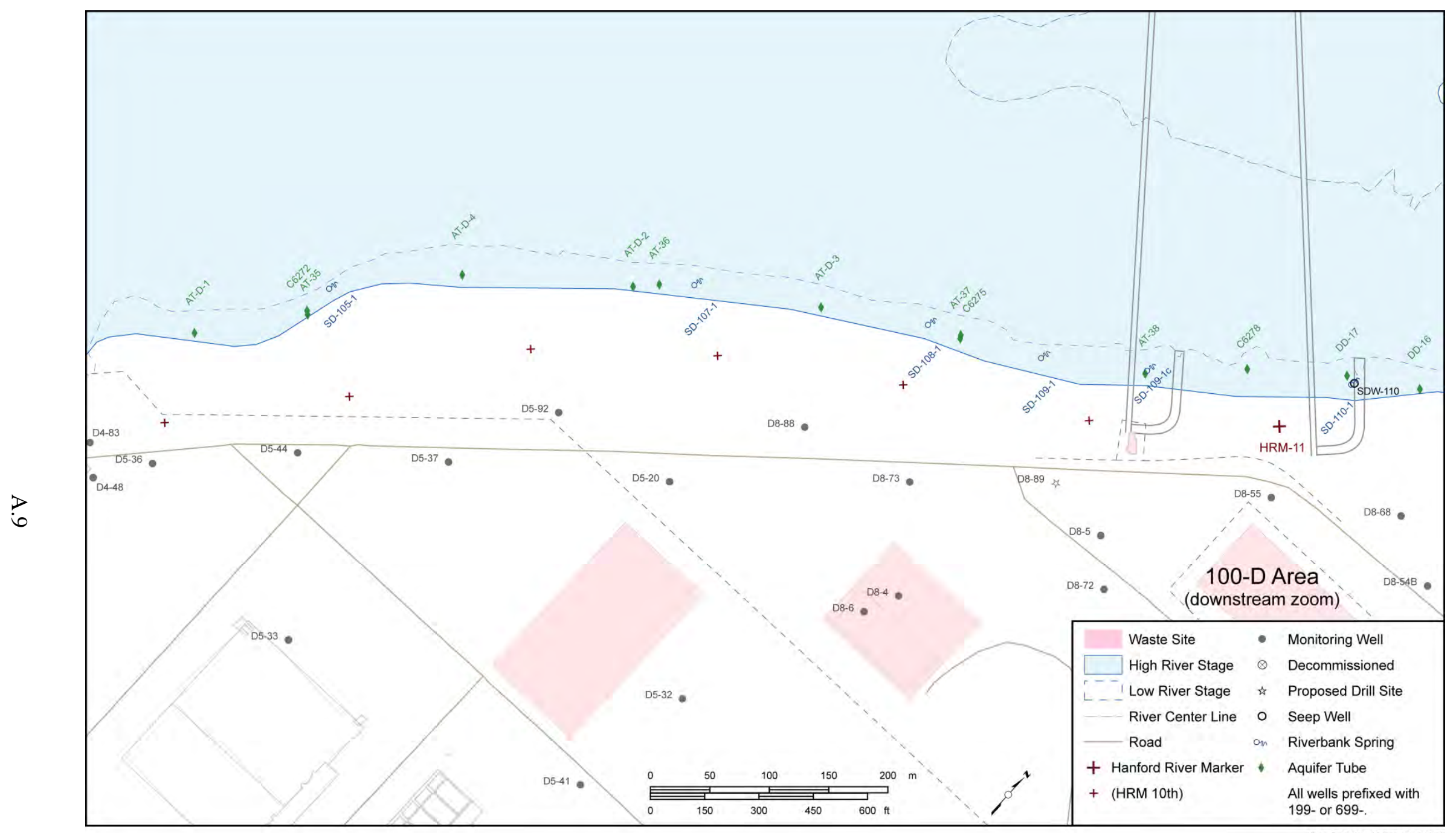

Figure A.8. Water Quality Monitoring Locations Along the Downstream Portion of the 100-D Area Shoreline 


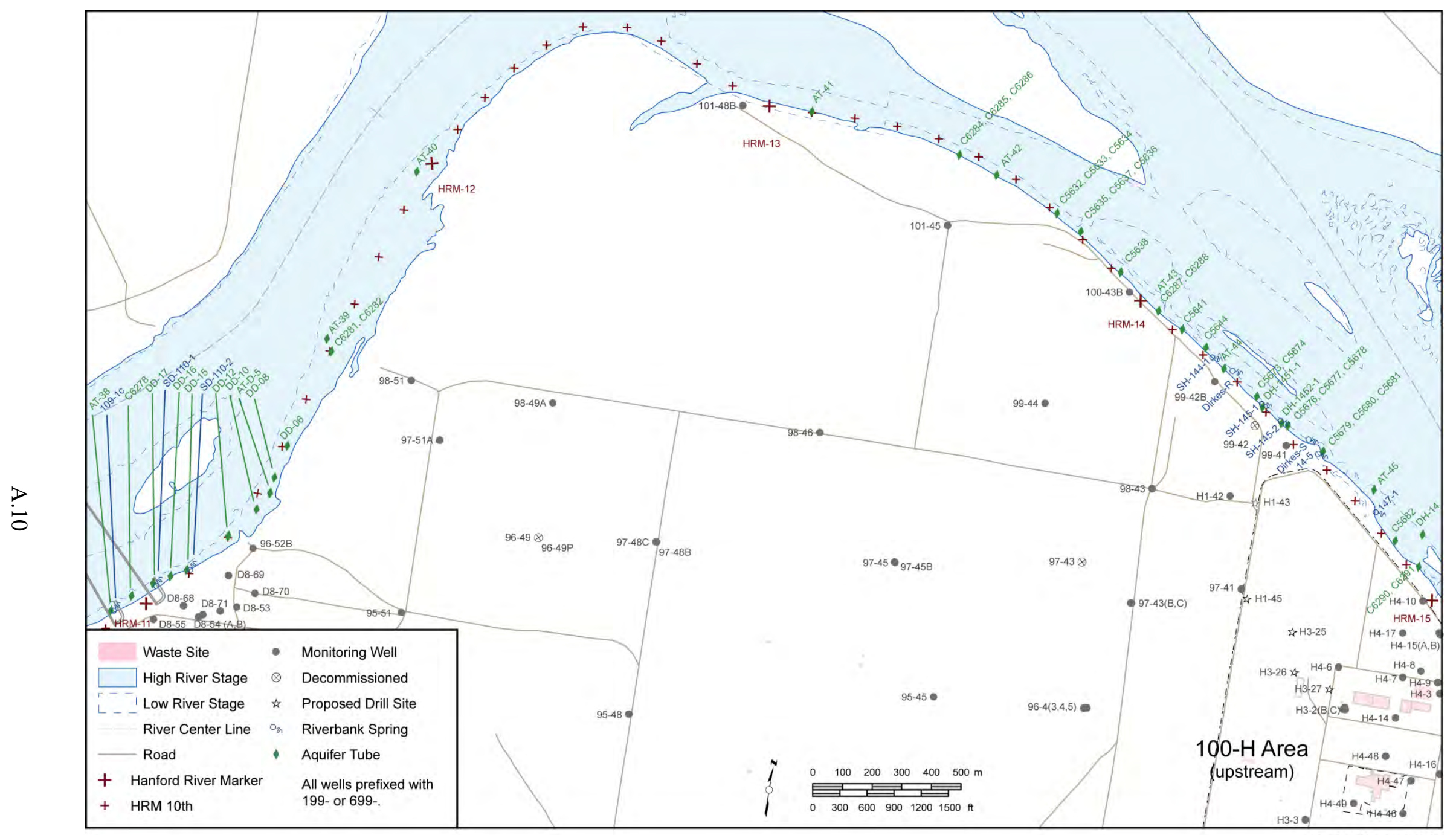

HReach_Up100H_krp_011910

Figure A.9. Water Quality Monitoring Locations Along the Shoreline from 100-D to 100-H Areas 


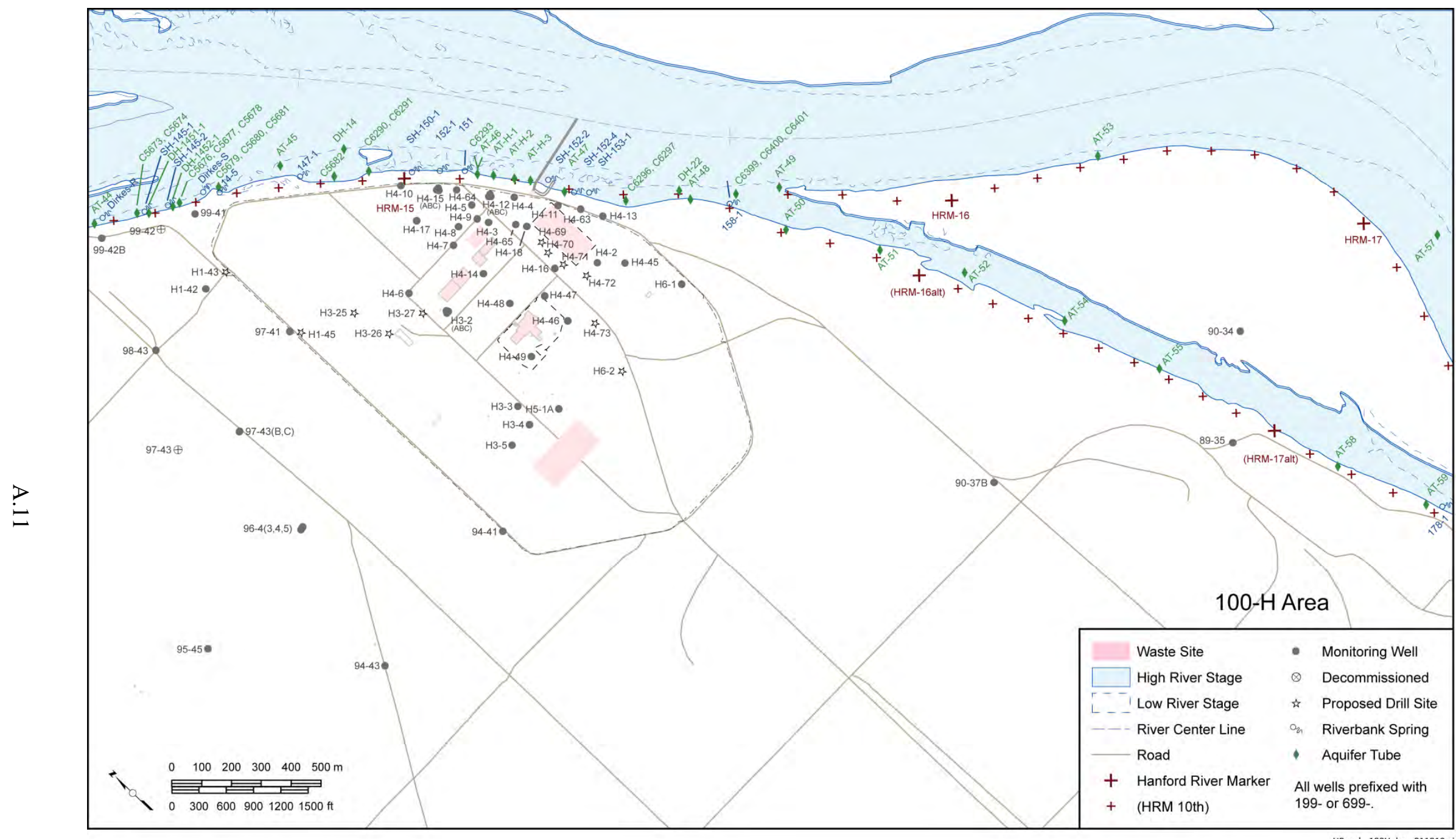

Figure A.10. Water Quality Monitoring Locations Along the 100-H Area Shoreline 


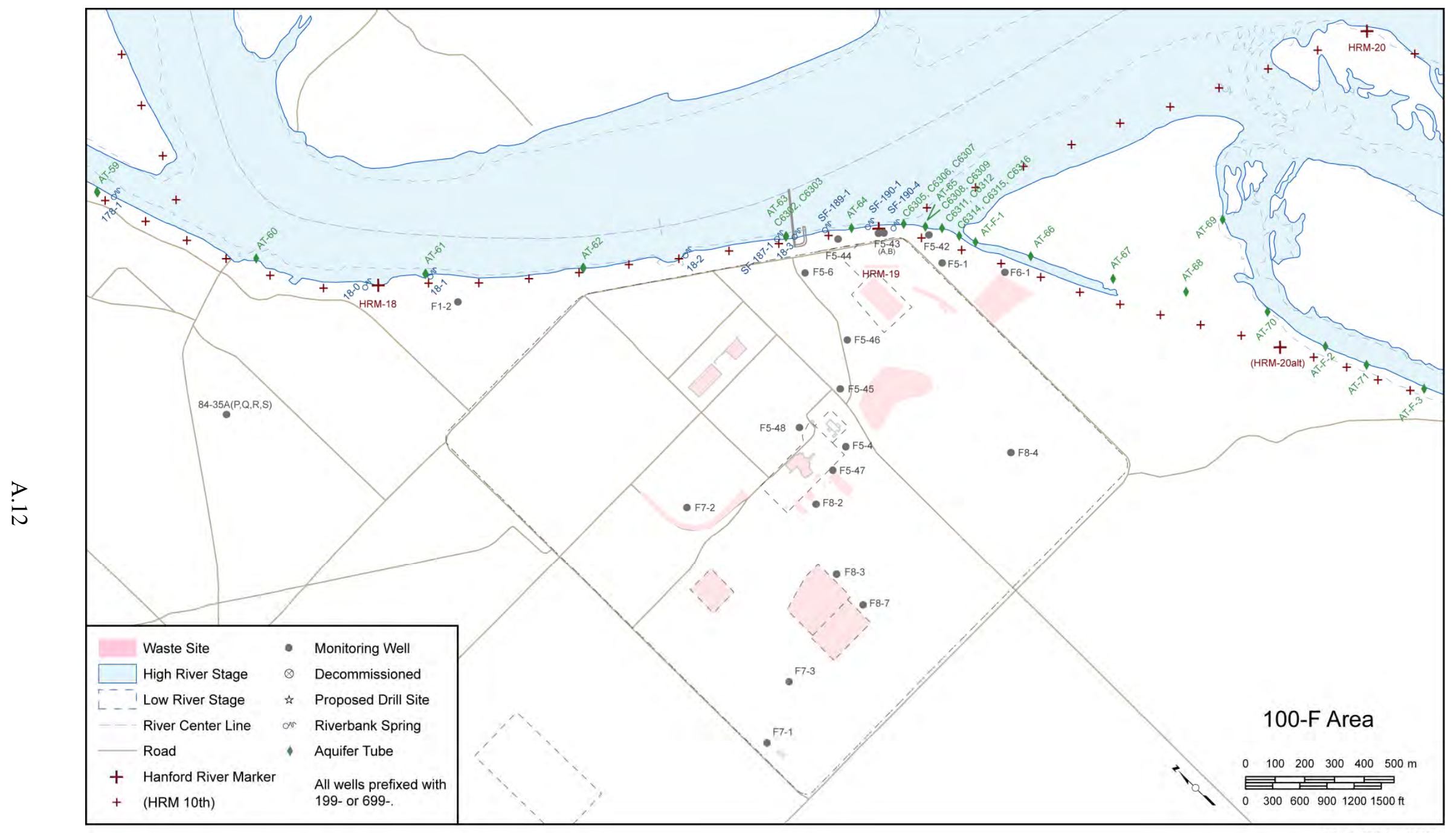

Figure A.11. Water Quality Monitoring Locations Along the 100-F Area Shoreline 


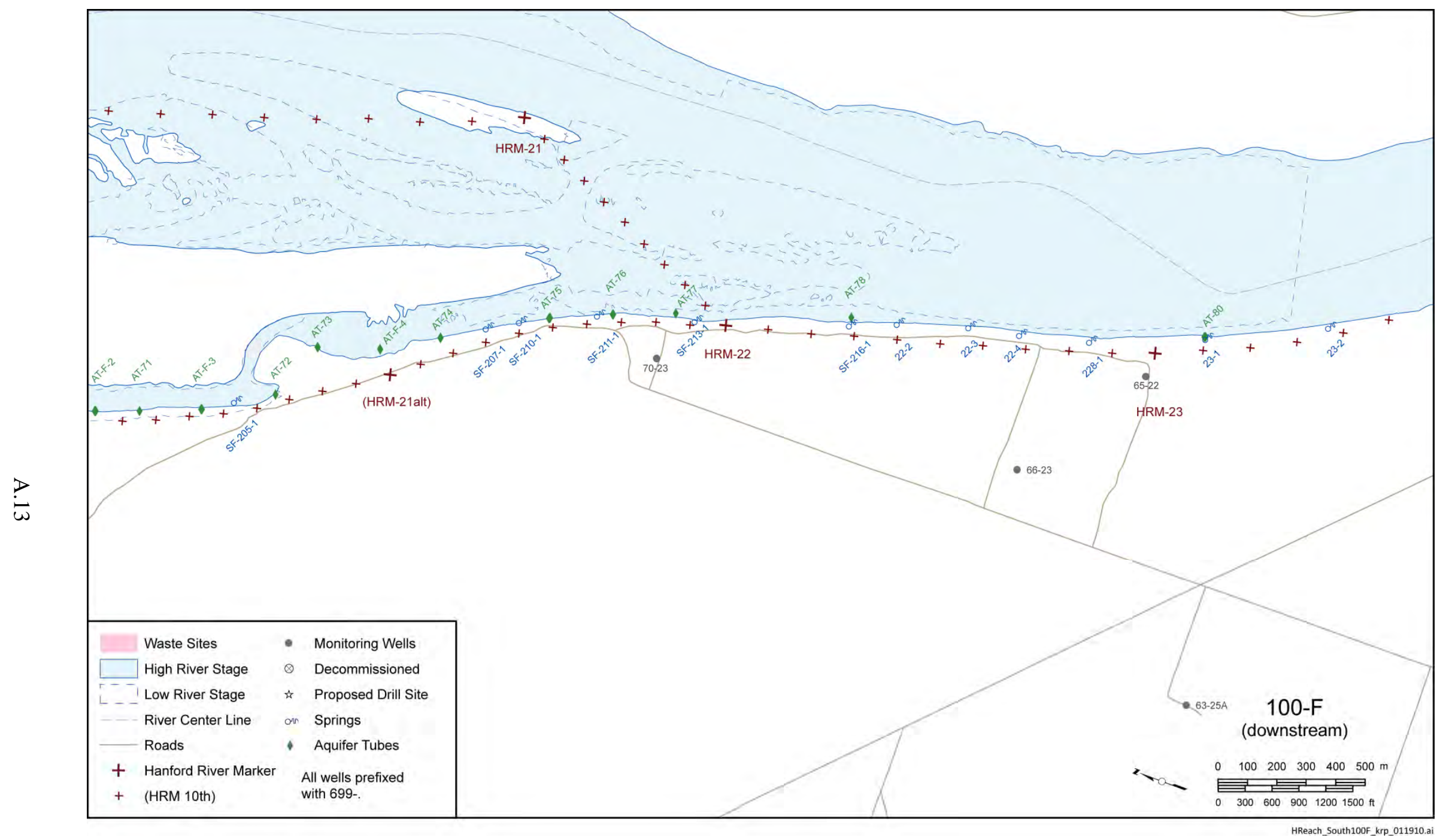

Figure A.12. Water Quality Monitoring Locations Along the Shoreline Downstream from 100-F Area 


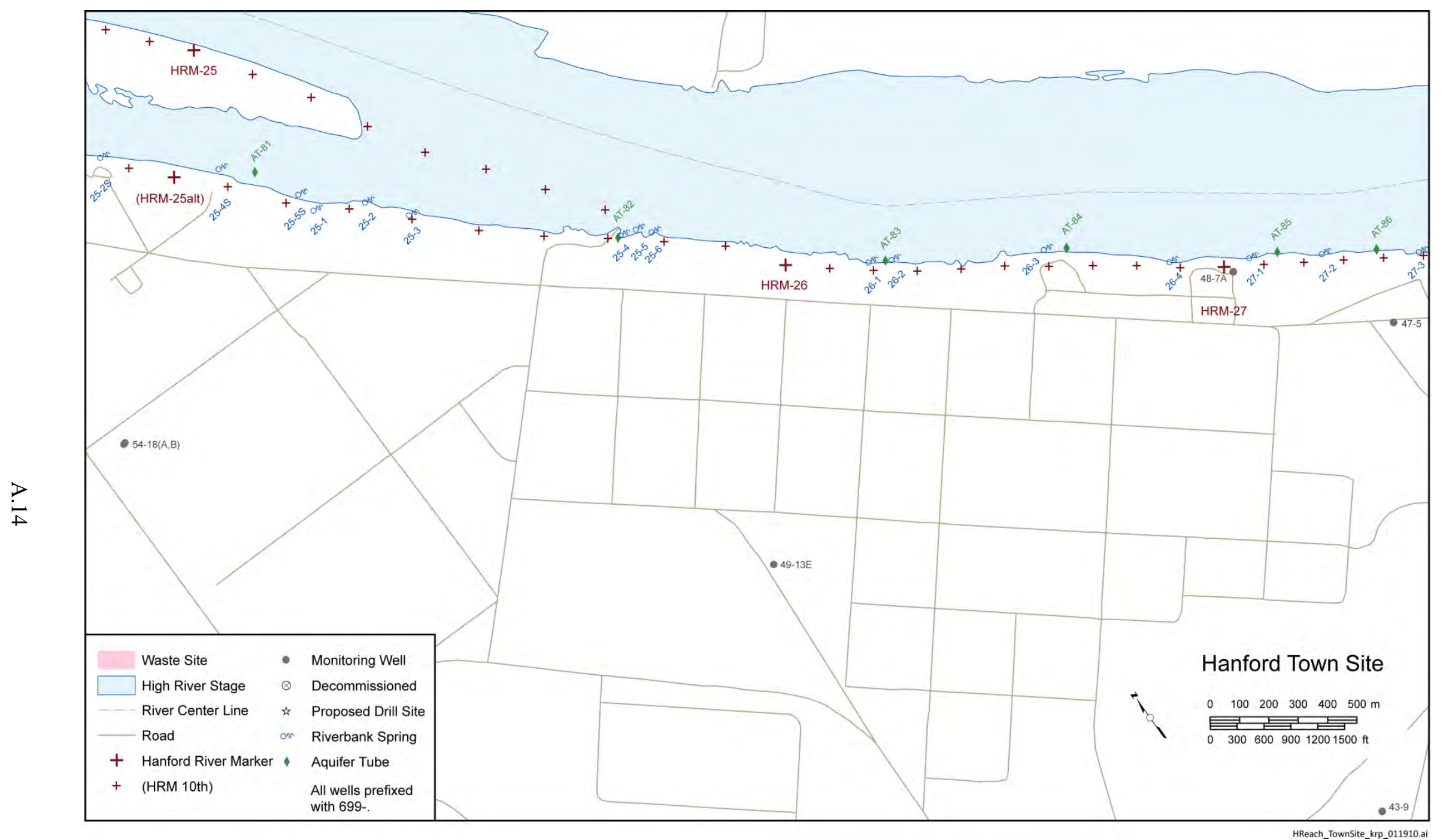

Figure A.13. Water Quality Monitoring Locations Along the Hanford Town Site Shoreline 


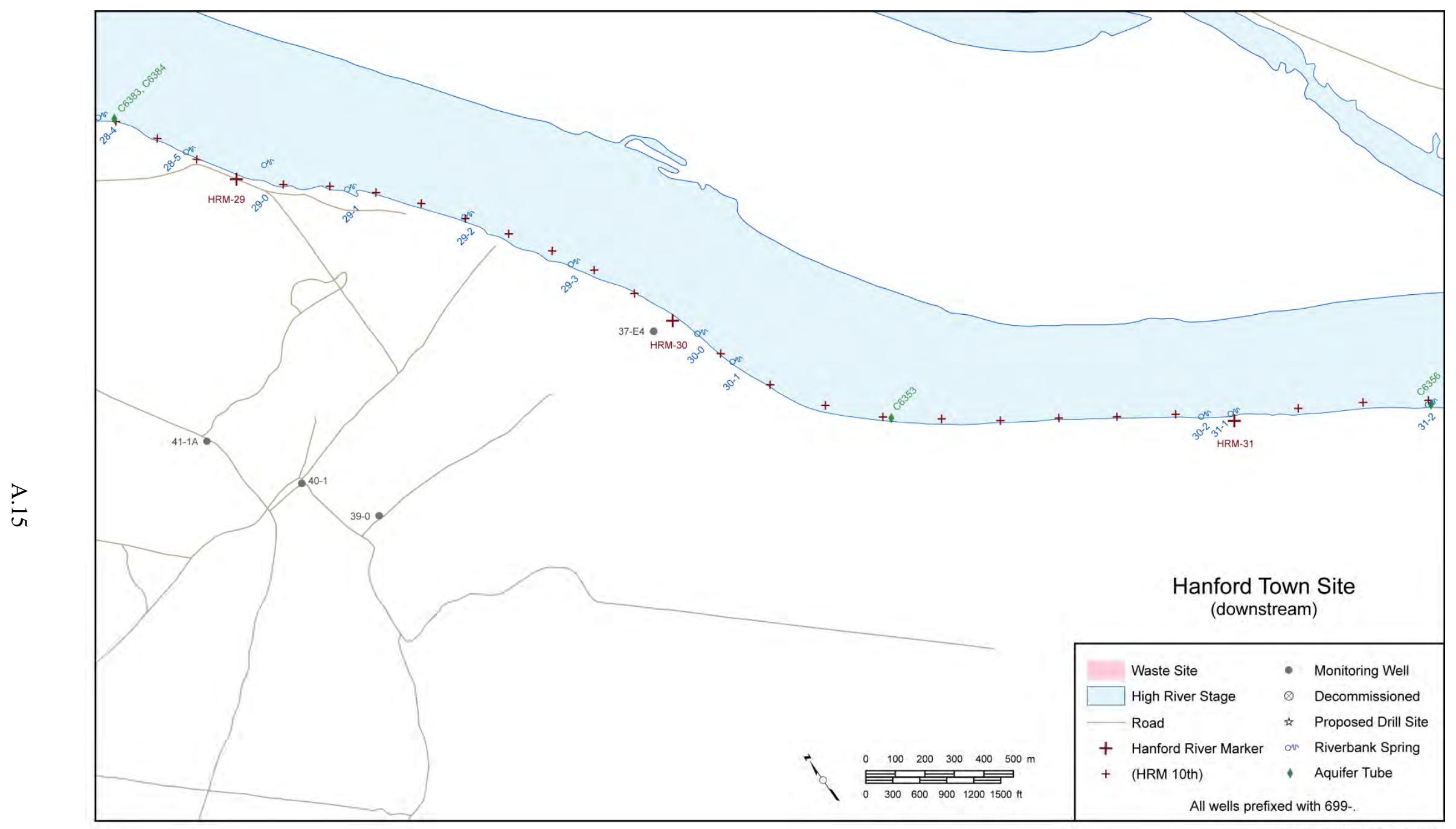

Figure A.14. Water Quality Monitoring Locations Along the Shoreline Downstream from the Hanford Town Site Shoreline 


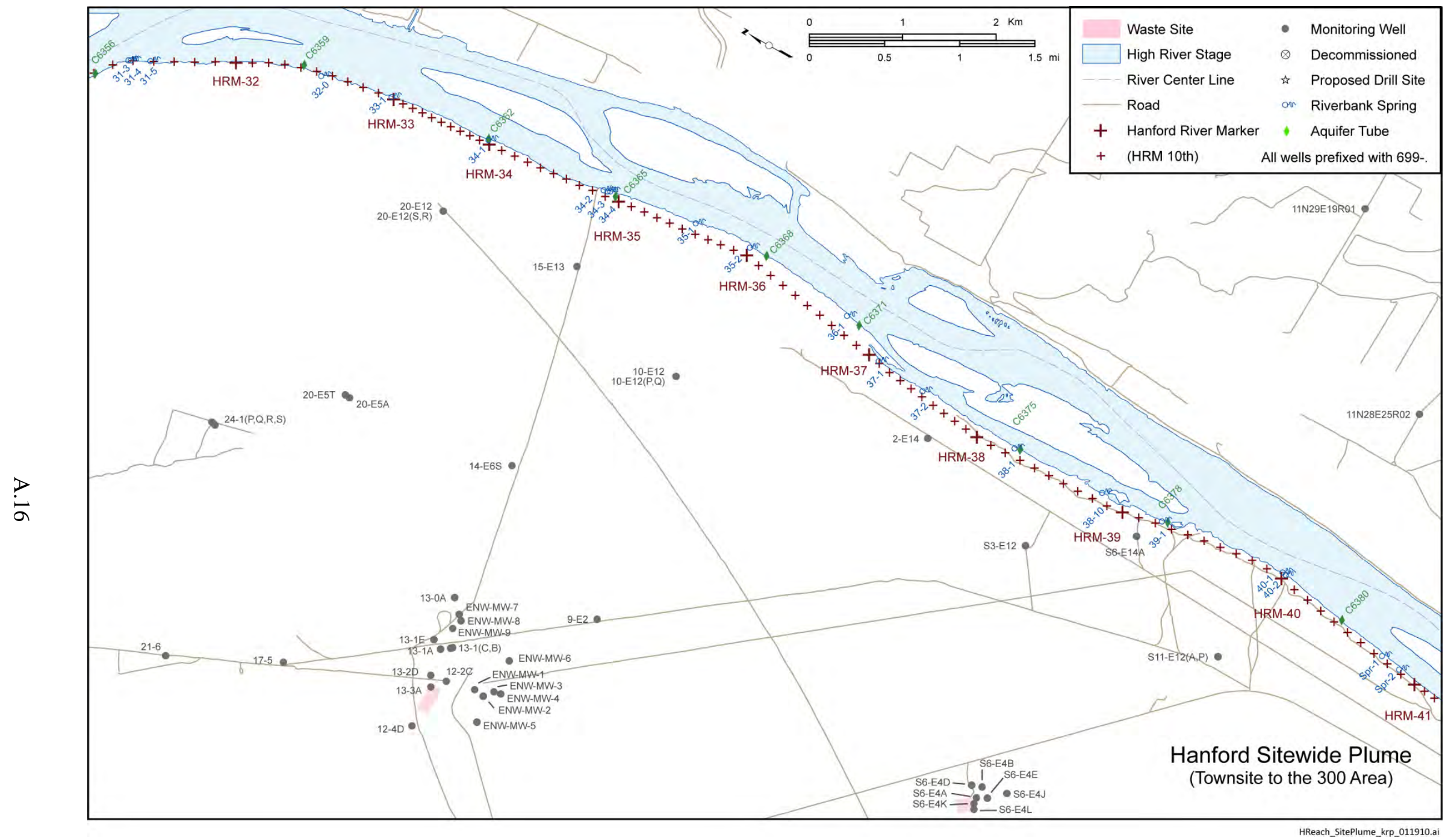

Figure A.15. Water Quality Monitoring Locations Along the Shoreline Impacted by the Hanford Sitewide Groundwater Plume 


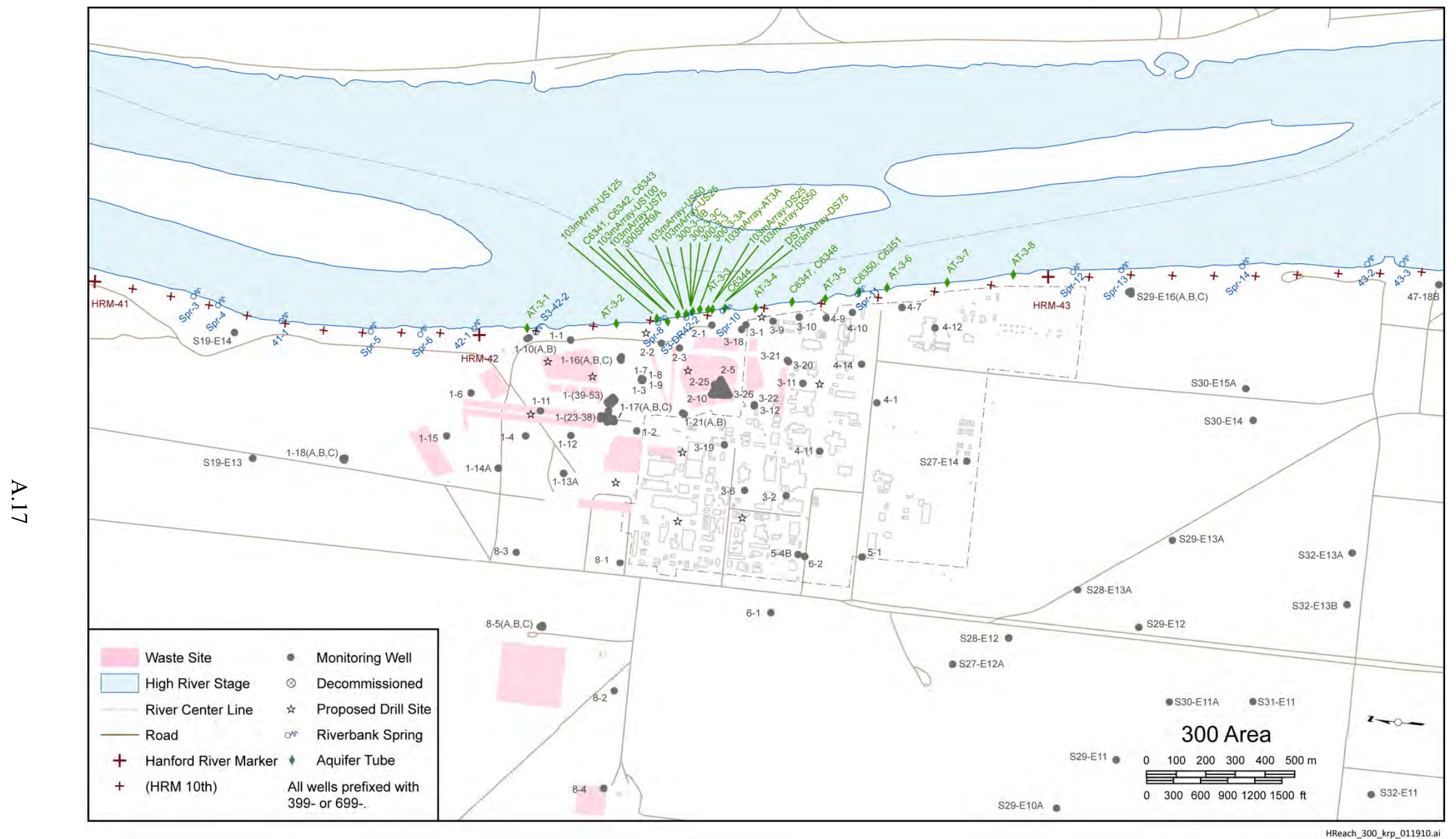

Figure A.16. Water Quality Monitoring Locations Along the 300 Area Shoreline 


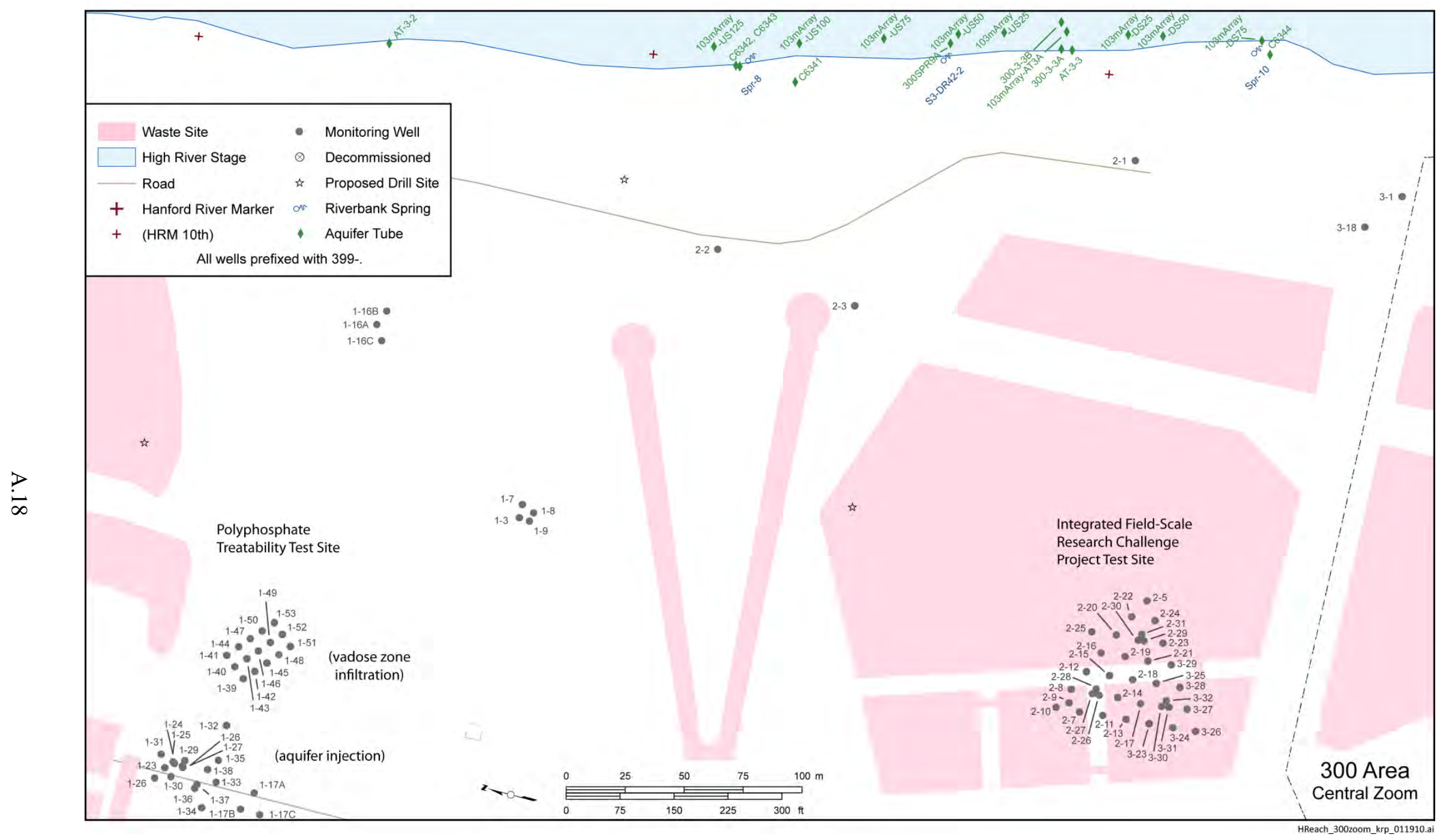

Figure A.17. Water Quality Monitoring Locations Along the Central Portion of the 300 Area Shoreline 


\section{Distribution}

No. of

Copies

7 U.S. Department of Energy, Richland Operations Office

J.A. Hansen

R.D. Hildebrand

J.G. Morse

J.P. Sands

K.M. Thompson

D.C. Ward

Public Reading Room

5 CH2M Hill Plateau Remediation Company

J.V. Borghese

H3-21

M.J. Hartman

R3-50

R.O. Mahood

R3-50

J.T. Rieger

H8-51

W.D. Webber

H8-51

2 Washington Closure Hanford, LLC

L.C. Hulstrom

H4-22

R.K. Zufelt

H4-22
No. of

Copies

16 Pacific Northwest National Laboratory

E.V. Arntzen K6-85

L.E. Bisping K6-75

R.L. Dirkes K6-75

B.G. Fritz K6-75

J.S. Fruchter K6-96

D.R. Geist K6-85

T.P. Hanrahan K6-85

K.R. Parker K6-96

R.E. Peterson (3) K6-75

G.W. Patton K6-75

T.M. Poston K6-75

Hanford Technical Library P8-55

1 Washington State Department of Ecology

$\begin{array}{ll}\text { D. Goswami H0-57 } & \text { HOmi }\end{array}$

1 U.S. Environmental Protection Agency

L.E. Gadbois $\quad$ B1-46

1 Washington State Department of Health

M. Priddy

B1-42

2 Other

Brett Tiller

Environmental Assessment Services

P.O. Box 265

Richland, WA 99352

Dan Tyler

Freestone Environmental Services

1100 Jadwin Ave. Suite 250

Richland, WA 99352-3425 




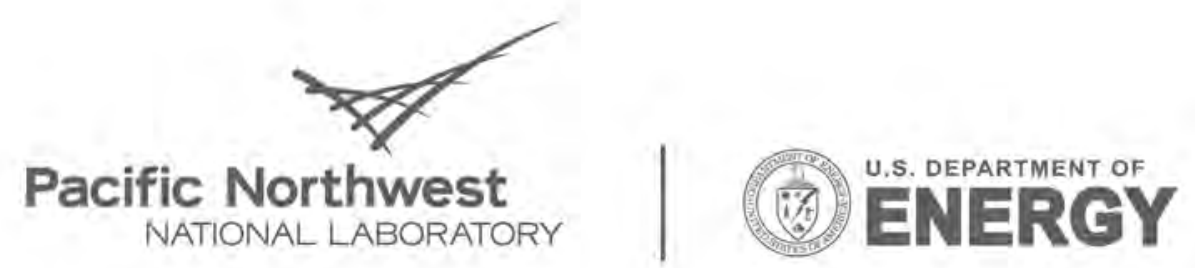

Proudly Operated by Battelle Since 1965

902 Battelle Boulevard

P.O. Box 999

Richland, WA 99352

1-888-375-PNNL (7665)

www.pnl.gov 